\title{
Asset Allokationsentscheidungen auf Basis höherer Momente und impliziter Informationen
}

\author{
Dissertation \\ zur Erlangung des wirtschaftswissenschaftlichen Doktorgrades \\ der Wirtschaftswissenschaftlichen Fakultät \\ der Georg August Universität Göttingen
}

vorgelegt von

Dipl.-Math. Felix Holger Brinkmann

aus Warendorf

Göttingen, 2014 
Erstgutachter: Prof. Dr. Olaf Korn

Georg August Universität Göttingen

Platz der Göttinger Sieben 3

37073 Göttingen

Zweitgutachter: Prof. Dr. Alexander Kempf

Universität zu Köln

Universitätsstraße 24

50923 Köln

Drittgutachter: Prof. Dr. Stefan Dierkes

Georg August Universität Göttingen

Platz der Göttinger Sieben 3

37073 Göttingen

Tag der mündlichen Prüfung: 14. Februar 2014 


\section{Inhaltsverzeichnis}

Tabellenverzeichnis $\quad$ iii

Abbildungsverzeichnis $\quad$ vi

1 Einleitung und Motivation der zentralen Forschungsfragen 1

1.1 Asset Allokation mit höheren Momenten . . . . . . . . . . . . . 2

1.2 Historische Schätzverfahren und der Umgang mit Schätzfehlern . . . . . . 5

1.3 Implizite Informationen in Optionsmarktdaten . . . . . . . . . . . . . 9

1.4 Forschungsfragen und Gang der Untersuchung . . . . . . . . . . . . . . 13

2 Das Problem der Asset Allokation $\quad 17$

2.1 Optimale Vermögensverteilung bei Berücksichtigung höherer Momente . . . 17

2.2 Konkretisierung der Investoren und deren Handelsmöglichkeiten . . . . . . 21

2.2.1 Spezifikation der betrachteten Investoren . . . . . . . . . . . 21

2.2.2 Annahmen an die Handelsmöglichkeiten der Investoren . . . . . . . 25

3 Strategische Asset Allokation mit impliziten Verteilungen 27

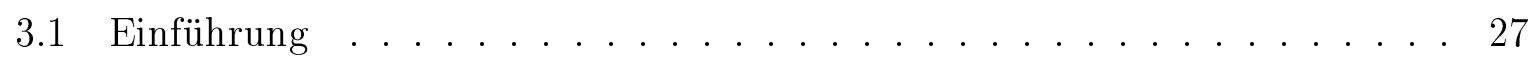

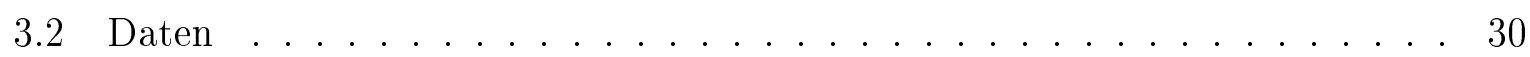

3.3 Implizite Verteilungen aus Optionsmarktdaten . . . . . . . . . . . . . 32

3.4 Methodik der empirischen Studie . . . . . . . . . . . . . 45

3.4.1 Bestimmung der optimalen Asset Allokation . . . . . . . . . . . . . . 46

3.4 .2 Performance-Kennzahlen . . . . . . . . . . . . . . . . 47

3.4 .3 Benchmark Strategien . . . . . . . . . . . . . . . . 49

3.5 Ergebnisse der empirischen Studie . . . . . . . . . . . . . . 51

3.5.1 Gründe für die Out-Performance der impliziten Strategie gegenüber den historischen Benchmark Strategien . . . . . . . . . . . 55

3.5.2 Analyse der Komposition des riskanten Anteils der aktiven Strategien in der Asset Allokation . . . . . . . . . . . . . . . . . 57

3.5.3 Berücksichtigung von Transaktionskosten . . . . . . . . . . . 60

3.6 Diskussion . . . . . . . . . . . . . . . . . . . 62 
4 Risikoadjustierte modellfreie implizite Momente 65

4.1 Einführung . . . . . . . . . . . . . . . . . 6 65

4.2 Herleitung risikoadjustierter modellfreier impliziter Momente . . . . . . . 67

4.3 Daten . . . . . . . . . . . . . . . . . . 80

4.4 Methodik und Design der empirischen Studie . . . . . . . . . . . . . 81

4.5 Konsequenzen aus der Risikoadjustierung . . . . . . . . . . . . . . 82

4.5.1 Wirkung der Risikoadjustierung auf OTM-Optionen . . . . . . . . . 82

4.5.2 Die Markt-Risikoprämie . . . . . . . . . . . . . . 84

4.5.3 Wirkung der Risikoadjustierung auf Varianz, Schiefe und Kurtosis . 85

4.6 Fähigkeit zur Prognose realisierter Momente . . . . . . . . . . . . . . . 94

4.6.1 Ergebnisse für die Volatilität . . . . . . . . . . . . . . 95

4.6.2 Ergebnisse für Schiefe und Kurtosis . . . . . . . . . . . . . . . 98

4.6.3 Ergebnisse für Subperioden . . . . . . . . . . . . . . . 99

4.6.4 Ergebnisse für zeitvariable Risikoaversionen . . . . . . . . . . . 101

4.7 Diskussion . . . . . . . . . . . . . . . . . . . . 105

5 Taktische Asset Allokation mit impliziten höheren Momenten 109

5.1 Einführung . . . . . . . . . . . . . . . . . . . 109

5.2 Vollständig implizite Schätzer für Matrizen höherer Ordnung . . . . . . . 112

5.2.1 Constant-Correlation-Ansatz . . . . . . . . . . . . . . . 112

5.2 .2 Annahme eines Ein-Faktor-Modells . . . . . . . . . . . . . 117

5.3 Daten . . . . . . . . . . . . . . . . . . . . 121

5.4 Methodik der empirischen Studie . . . . . . . . . . . . . . . 123

5.4.1 Bestimmung der optimalen Asset Allokation . . . . . . . . . . . 123

5.4 .2 Benchmark Strategien . . . . . . . . . . . . . . 125

5.4.3 Performance-Kennzahlen . . . . . . . . . . . . . . 127

5.5 Ergebnisse der empirischen Studie . . . . . . . . . . . . . . . . . . 129

5.5.1 Korrelationen höherer Momente . . . . . . . . . . . . . . . 130

5.5.2 Ergebnisse der Portfoliostudie . . . . . . . . . . . . . 135

5.5.3 Berücksichtigung von Transaktionskosten . . . . . . . . . . . . 141

5.5.4 Unterteilung des out-of-sample Zeitraums nach der realisierten Volatilität des Marktes . . . . . . . . . . . . . . . 144

5.5.5 Nutzen von höheren impliziten Momenten . . . . . . . . . . . 150

5.6 Diskussion . . . . . . . . . . . . . . . . . . . . 155

$\begin{array}{lll}6 & \text { Schlussbetrachtung und Ausblick } & 157\end{array}$

Literaturverzeichnis 


\section{Tabellenverzeichnis}

2.1 Wirkung höherer Momente auf das Gewicht der riskanten Anlage . . . . 23

2.2 Nutzenverlust bei Vernachlässigung der höheren Momente . . . . . . . . . 24

3.1 Einfluss des Glättungsparameters auf die Momente der Verteilung . . . . . 36

3.2 Einfluss der Risikoadjustierung auf die Momente des Kurses . . . . . . . . 39

3.3 Auswertungen des Likelihood Ratio Tests für ausgewählte Risikoaversionsparameter . . . . . . . . . . . . . . . . . . . . 42

3.4 Einfluss der Risikoadjustierung auf die Momente der risikoneutralen Verteilung . . . . . . . . . . . . . . . . . . 42

3.5 Sicherheitsäquivalente, Excess Returns und realisierte Volatilitäten für die riskanten Anlageklassen . . . . . . . . . . . . . . . . 49

3.6 Übersicht der betrachteten aktiven und passiven Strategien der empirischen Studie . . . . . . . . . . . . . . . . 50

3.7 Sicherheitsäquivalente, Excess Returns und realisierte Volatilitäten der aktiven Strategien . . . . . . . . . . . . . . . . . 52

3.8 Sicherheitsäquivalente, Excess Returns und realisierte Volatilitäten der passiven Strategien . . . . . . . . . . . . . . . . 53

3.9 Sicherheitsäquivalente, Excess Returns und realisierte Volatilitäten der aktiven historischen Strategien mit impliziten Erwartungswerten . . . . . . 55

3.10 Deskriptive Statistiken für das Gewicht der risikofreien Anlage . . . . . . . 56

3.11 Durchschnittliche Komposition des riskanten Anteils der Asset Allokation . 58

3.12 Deskriptive Statistiken für die Differenz der normierten Gewichte zwischen den Risikoaversionen 4 und 8 der riskanten Assets . . . . . . . . . . . . . 59

3.13 Turnover sowie um Transaktionskosten bereinigte Sicherheitsäquivalente und Excess Returns der aktiven Strategien . . . . . . . . . . . . . . . 61

4.1 Parameter für die Risikoadjustierung bei konstanter absoluter Risikoaversion (CARA) und konstanter relativer Risikoaversion (CRRA) . . . . . . 72

4.2 Deskriptive Statistiken für die approximierten (impliziten) Excess Returns 84 
4.3 Erwartungswerte und Standardabweichungen für die Differenz aus risikoadjustiertem und implizitem Moment . . . . . . . . . . . . . . 88

4.4 Bestimmtheitsmaße für die Erklärung der Differenz aus risikoadjustiertem und implizitem Moment durch die Approximation bis zur ersten und zweiten Ordnung . . . . . . . . . . . . . . . . . . . . . 93

4.5 Deskriptive Statistiken und Ergebnisse des Regressionsmodells für die realisierten, impliziten und risikoadjustierten Volatilitäten . . . . . . . . . 95

4.6 Deskriptive Statistiken und Ergebnisse des Regressionsmodells für die realisierten, impliziten und risikoadjustierten Schiefen und Kurtosin . . . . . . 98

4.7 Ergebnisse des Regressionsmodells für die realisierten, impliziten und risikoadjustierten höheren Momente für zwei Subperioden . . . . . . . . . . . 100

4.8 Deskriptive Statistiken für die Excess Returns und die risikoadjustierten höheren Momenten für geschätzte Risikoaversionsparameter und Ergebnisse des Regressionsmodells . . . . . . . . . . . . . . . . 103

4.9 Deskriptive Statistiken für die risikoadjustierten Momente für die zeitvariablen Risikoaversionen aus Duan und Zhang (2010) und Ergebnisse des Regressionsmodells . . . . . . . . . . . . . . . . . 104

5.1 Anzahl der Komomente und der Korrelationen höherer Ordnung in den Matrizen $M_{2}, M_{3}$ und $M_{4} \ldots \ldots \ldots \ldots 117$

5.2 Zeitraum und Ticker der Unternehmen im DJIA . . . . . . . . . . . . . 122

5.3 Deskriptive Statistiken für die optimalen Shrinkageintensitäten für die strukturierten Matrizen $M_{2}, M_{3}$ und $M_{4} \ldots \ldots \ldots 126$

5.4 Übersicht der betrachteten aktiven und passiven Anlagestrategien der empirischen Studie . . . . . . . . . . . . . . . . . . . 127

5.5 Mittlere Differenz und absoluter Fehler aus der Differenz der realisierten Korrelationen und den geschätzten Werten sowie das Ergebnis des Regressionsmodells . . . . . . . . . . . . . . . . . . . . 133

5.6 MUGs der passiven und historischen Strategien gegenüber der impliziten Strategie auf Basis des Constant-Correlation-Ansatzes . . . . . . . . . . 136

5.7 Excess Returns und realisierte Volatilitäten der passiven, historischen und impliziten Strategien auf Basis des Constant-Correlation-Ansatzes . . . . . 138

5.8 MUGs der passiven und historischen Strategien gegenüber der impliziten Strategie auf Basis der Annahme eines Ein-Faktor-Modells . . . . . . . . 140

5.9 Turnover und um Transaktionskosten bereinigte MUGs der passiven und historischen Strategien gegenüber der impliziten Strategie auf Basis des Constant-Correlation-Ansatzes . . . . . . . . . . . . . . . . . 141 
5.10 Turnover und um Transaktionskosten bereinigte MUGs der passiven und historischen Strategien gegenüber der impliziten Strategie auf Basis der Annahme eines Ein-Faktor-Modells . . . . . . . . . . . . . . . . 143

5.11 MUGs der passiven und historischen Strategien gegenüber der impliziten Strategie auf Basis des Constant-Correlation-Ansatzes für zwei Subperioden 146

5.12 Excess Returns und realisierte Volatilitäten der passiven, historischen und impliziten Strategien auf Basis des Constant-Correlation-Ansatzes für zwei Subperioden . . . . . . . . . . . . . . . . . . . . 147

5.14 MUGs der passiven und historischen Strategien gegenüber der impliziten Strategie auf Basis der Annahme eines Ein-Faktor-Modells für zwei Subpe-

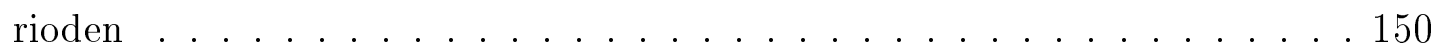

5.15 MUGs für die implizite Strategie Impl_CC gegenüber dem MinimumVarianz-Portfolio auf Basis des Constant-Correlation-Ansatzes für den gesamten Zeitraum und zwei Subperioden . . . . . . . . . . . . . . . . 151

5.16 Differenzen der Excess Returns und der realisierten Volatilität zwischen der impliziten Strategie Impl_CC und dem Minimum-Varianz-Portfolio auf Basis des Constant-Correlation-Ansatzes für den gesamten Zeitraum und zwei Subperioden . . . . . . . . . . . . . . . . . . . 152

5.17 Durchschnittlicher absoluter Abstand zwischen dem gleichgewichteten In$\operatorname{dex}(1 / \mathrm{N})$ und den Gewichten der impliziten Strategie Impl_CC und des Minimum-Varianz-Portfolios auf Basis des Constant-Correlation-Ansatzes für den gesamten Zeitraum und zwei Subperioden . . . . . . . . . . . . . 153

5.18 MUGs für die implizite Strategie Impl_SF gegenüber dem Minimum-VarianzPortfolio auf Basis der Annahme eines Ein-Faktor-Modells für den gesamten Zeitraum und zwei Subperioden . . . . . . . . . . . . . . 154 


\section{Abbildungsverzeichnis}

3.1 Kumulierte Excess Returns der riskanten Anlagewerte . . . . . . . . . . . . 32

3.2 Marktpreise und implizite Volatilitäten von europäischen Call-Optionen auf

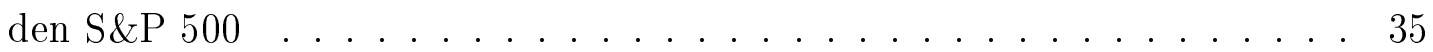

3.3 Einfluss des Glättungsparameters auf die risikoneutrale Dichte . . . . . . 36

3.4 Einfluss der Risikoadjustierung auf die risikoneutrale Dichte . . . . . . . . 43

3.5 Geschätzte Risikoaversionsparameter für die riskanten Anlagewerte . . . . 44

3.6 2-dimensionale physische Dichte für den S\&P 500 und den TNX Index. . . 46

4.1 Einfluss der Risikoadjustierung auf die EDAP's . . . . . . . . . . . 83

4.2 Approximierte (implizite) Excess Returns . . . . . . . . . . . . . . . . 84

4.3 Differenz zwischen der risikoadjustierten Volatilität, Schiefe und Kurtosis und den impliziten Momenten . . . . . . . . . . . . 86

4.4 Realisierte, implizite und risikoadjustierte Volatilität . . . . . . . . . . 95

4.5 Realisierte, implizite und risikoadjustierte Schiefe und Kurtosis . . . . . . . 97

4.6 Risikoaversionen des S\&P 500 aus Duan und Zhang (2010) und geschätzte Aversionen aus historischen Daten der letzten 120 Monate . . . . . . . . . 102

5.1 Realisierte, historisch und implizit geschätzte Korrelationen höherer Momente131

5.2 Differenzen aus der implizit geschätzten Korrelation $\rho^{M_{2}}$ und der impliziten

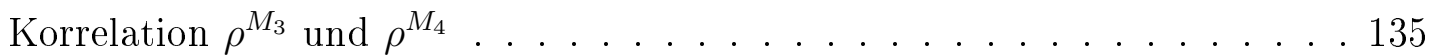




\section{Kapitel 1}

\section{Einleitung und Motivation der zentralen Forschungsfragen}

Die Allokation von Vermögen auf unterschiedliche Anlagewerte und die Bildung eines entsprechenden Portfolios ist von zentraler Bedeutung für Privatpersonen, Unternehmen und für private sowie staatliche Anlagefonds. Beispielhaft seien hier der Aufbau privater Altersversorgungen, die Anlagestrategien von Fondmanagern oder die Vermögensverwaltung von Versicherungskonzernen genannt. Für die Finanzwirtschaft stellen die Asset Allokation und die Portfoliotheorie einen zentralen Forschungsgegenstand dar.

Der Beginn der modernen Portfoliotheorie, und damit der modernen finanzwirtschaftlichen Forschung, kann auf die fundamentale Arbeit von Markowitz (1952) zurückgeführt werden. In seinem Beitrag betrachtet er neben der Rendite eines Investments erstmals auch das einzugehende Risiko und stellt es der Rendite gegenüber. Die aus diesem Ansatz abgeleiteten "optimalen" Vermögensaufteilungen sind bis heute Grundlage unterschiedlichster Investmentstrategien und im Interesse von Forschung und Praxis gleichermaßen.

Die von Markowitz (1952) ausgehende Portfoliotheorie wurde weiterentwickelt und kann in das übergeordnete Konzept der Erwartungsnutzentheorie eingebettet werden. Im Rahmen dieser sind für die Portfoliotheorie zwei Aspekte fundamental: zum einem muss der betrachtete Investor mit Hilfe einer fundierten Theorie in seinen Präferenzen beschrieben werden, zum anderen müssen Erwartungen über die zukünftige Entwicklung der zur Verfügung stehenden Anlagewerte gebildet werden. Die optimale Asset Allokation erfolgt, indem der Investor sein Vermögen entsprechend seiner Präferenzen und Erwartungen auf die Anlagewerte verteilt, so dass der erwartete Nutzen aus dem Investment maximal ist. Entscheidend für den resultierenden Nutzen seiner Anlagestrategie ist einerseits der Genauigkeitsgrad der Erfassung seiner Präferenzen bei der Wahl seines Portfolios, 
andererseits die Güte der gebildeten Erwartungen über die zukünftige Entwicklung der Anlagewerte.

Die vorliegende Arbeit ist beiden Aspekten der Portfoliotheorie zuzuordnen und verbindet im Rahmen der Bestimmung der optimalen Asset Allokation eine exaktere Erfassung der Präferenzen des Investors mit der Erwartungsbildung auf Basis von (impliziten) Informationen aus derivativen Finanzinstrumenten.

Im folgenden Teil der Einleitung wird zunächst eine Einführung in die Portfoliotheorie mit einem Fokus auf die Erfassung von Präferenzen in der Asset Allokation gegeben, bevor deren Umsetzung mit Hilfe historischer Renditezeitreihen sowie der damit verbundenen theoretischen und praktischen Probleme diskutiert werden. Der darauffolgende Abschnitt motiviert die Verwendung impliziter Informationen aus Optionsmarktdaten für die Erwartungsbildung in der Asset Allokation. Die Einleitung schließt mit der Formulierung der zentralen Forschungsfragen und der Einordnung der empirischen Studien dieser Arbeit in die bestehende Literatur.

\subsection{Asset Allokation mit höheren Momenten}

In einer Situation, in der ein Investor eine Asset Allokation vornimmt, d. h. sein verfügbares Vermögen optimal auf eine gegebene Anzahl von Anlagewerten verteilen möchte, müssen die oben beschriebenen zwei Aspekte der Portfoliotheorie betrachtet werden. Es sei davon ausgegangen, dass sich der Investor bereits Erwartungen über die zukünftige Entwicklung der Anlagewerte gebildet hat und diese in Form einer (multidimensionalen) Wahrscheinlichkeitsverteilung bzw. der entsprechenden Momente der Verteilung vorliegen. Um nun zu seiner optimalen Asset Allokation zu kommen, muss der Investor zunächst seine Präferenzen bzgl. der Momente spezifizieren.

Falls der Investor seine Investmententscheidung ausschließlich auf Basis der ersten zwei Momente der Wahrscheinlichkeitsverteilung, also dem Erwartungswert und der Varianz, trifft, wird dies im Folgenden als wichtiger Spezialfall eines Investors mit $\mu-\sigma$ Nutzenfunktion bzw. als $\mu-\sigma$ Entscheider charakterisiert. ${ }^{1}$ Die optimalen Vermögensaufteilungen dieses Investors können als geschlossene Formeln dargestellt werden und haben große Bedeutung für Praxis und Forschung. Als wesentliche Forschungsgegenstände seien in die-

$1 \quad$ Für den weiteren Verlauf der Arbeit wird unter dem Begriff $\mu-\sigma$ Nutzenfunktion die Funktion $U$ in Abhängigkeit des Vermögens $W$, welche definiert ist durch:

$$
U(W)=E(W)-\gamma \cdot V A R(W)
$$

verstanden, wobei $\gamma$ den Grad der Risikoeinstellung des Investors widerspiegelt. 
sem Zusammenhang das Tangentialportfolio, bestimmt aus der Maximierung der Sharpe Ratio, sowie das globale Minimum-Varianz-Portfolio, ermittelt aus der Minimierung der Portfoliovarianz, genannt. ${ }^{2}$

Für Investoren mit allgemeineren Nutzenfunktionen lassen sich theoretisch neben Erwartungswert und Varianz auch Präferenzen für die höheren Momente Schiefe und Kurtosis bestimmen. ${ }^{3}$ Für diese Anleger sind die aus einer $\mu-\sigma$ Nutzenfunktion abgeleiteten Portfolios, wie das Tangentialportfolio, (ex ante) nicht mehr optimal und die Berücksichtigung der Präferenzen für Schiefe und Kurtosis in der Asset Allokation stellt folglich eine verbesserte Approximation der optimalen Vermögensaufteilung des Investors dar. ${ }^{4}$

Dass Schiefe und Kurtosis auch praktische Relevanz haben und bewertungsrelevante Informationen beinhalten, zeigen die Studien von Harvey und Siddique (2000) sowie Dittmar (2002). Erstere erweitern das Capital Asset Pricing Modell nach Sharpe (1964), Lintner (1965) und Mossin (1966) um die Koschiefe eines individuellen Assets mit dem Marktportfolio und ermitteln empirisch eine Risikoprämie für Schiefe. ${ }^{5}$ Die Autoren können in ihrer Studie ableiten, dass Investoren für zusätzliche positive Schiefe eine geringere erwartete Rendite verlangen. Dittmar (2002) leitet auf Basis eines Asset-Pricing-Modells mit nicht linearen Pricing-Kernels empirisch her, dass Asset-Preise neben der Kovarianz auch durch Koschiefen und Kokurtosin beeinflusst werden und dass Investoren avers gegenüber Kurtosisrisiken sind. ${ }^{6}$ Ferner ist abzulehnen, dass Assetrenditen normalverteilt sind und somit weder Schiefe noch (Excess-) Kurtosis besitzen. Vielmehr werden Renditeverteilungen mit asymmetrischen Dichten (Schiefe ungleich null) und Fat-Tails (Kurtosis größer drei) beobachtet. ${ }^{7}$

Die Hinzunahme von Schiefe und Kurtosis in der Asset Allokation führt in der Regel zu einer Aufteilung des Vermögens, die sich von derjenigen unterscheidet, die auf Basis von Erwartungswert und Varianz getroffen wurde. Jedoch ist zu beachten, dass ein Investor trotz Präferenzen für höhere Momente auch stets die Möglichkeit hat, die höheren Momente in seiner Asset Allokation zu vernachlässigen, und die Investitionsentscheidung an einem $\mu-\sigma$ Entscheider auszurichten. Gründe, dies zu tun, könnten unter anderem sein, dass der Investor sich seiner Erwartungsbildung unsicher ist oder die Komplexität der

2 Für eine komprimierte Einführung in die Portfolioselektion für Investoren mit $\mu-\sigma$ Nutzenfunktionen siehe z. B. Memmel (2004) oder Saßning (2012).

$3 \quad$ Vgl. Kraus und Litzenberger (1976) und Scott und Horvath (1980).

4 Die Berücksichtigung von höheren Momenten in der Portfolioselektion wurde bereits in Arditti (1967) und Samuelson (1970) diskutiert.

$5 \quad$ Vgl. auch Smith (2007).

6 Vgl. auch Chabi-Yo (2012) und Guidolin und Timmermann (2008).

7 Vgl. z. B. Harvey und Siddique (2000), Bekaert und Wu (2000) und die Literaturübersicht in Jondeau und Rockinger (2006). 
Asset Allokation reduzieren will. Kroll, Levy und Markowitz (1984) kommen in diesem Zusammenhang sogar zu dem Ergebnis, dass die Portfoliogewichte auf Basis einer $\mu$ $\sigma$ Nutzenfunktion eine fast optimale Lösung des Asset Allokationsproblems mit höheren Momenten darstellen.

Andere Studien widerlegen diese Ansicht. So zeigen Hong, Tu und Zhou (2007), dass bei Vernachlässigung der Asymmetrie der Renditeverteilung in einem bayesianischen Modellrahmen $^{8}$ ein Investor mit Disappointment Aversion nach Ang, Bekaert und Liu (2005) einen substanziellen ökonomischen Verlust erleidet. Jondeau und Rockinger (2006) unterstützen für einen Investor mit konstanter absoluter Risikoaversion dieses Ergebnis. Sie finden, dass im Gegensatz zur Asset Allokation auf Basis der ersten zwei Momente die Verwendung von Schiefe und Kurtosis bei der Optimierung eine gute Approximation des erwarteten Nutzens gewährleistet. Lediglich bei Renditeverteilungen, welche nur "leicht" von der Normalverteilung abweichen, ist der Nutzenverlust zu vernachlässigen. ${ }^{9}$ Guidolin und Timmermann (2008) stellen im Rahmen der internationalen Diversifikation die allein auf von Erwartungswert und Varianz basierende Asset Allokation derjenigen bei entsprechender Hinzunahme von Schiefe und Kurtosis gegenüber. Ihr empirisches Ergebnis hat weitreichende Relevanz für die Praxis, da insbesondere der Anteil an Auslandsinvestitionen stark unter den Vermögensaufteilungen variiert.

Dass tatsächlich höhere Momente in der Portfoliowahl Berücksichtigung finden, zeigen Mitton und Vorkink (2007). Die empirische Auswertung eines umfangreichen Datensatzes von Depots individueller Investoren legt dar, dass die Abweichung von effizienten Portfolios im Sinne eines $\mu-\sigma$ Entscheiders durch die Hinzunahme von zusätzlicher Portfolioschiefe erklärt werden kann. ${ }^{10}$

Zusammenfassend hat die Berücksichtigung von Schiefe und Kurtosis in der Asset Allokation theoretische und praktische Relevanz. Bezüglich der Vorteilhaftigkeit im Rahmen eines out-of-sample Settings, bei welchem neben der Berücksichtigung der höheren Momente auch die Güte der Erwartungsbildung zum Tragen kommt, sind die Ergebnisse bisheriger Forschung hingegen uneinheitlich. So zeigen Adler und Kritzman (2007), dass sich die in-sample Vorteilhaftigkeit auch out-of-sample durchsetzt, jedoch basiert die empirische Studie auf Hedge-Fund-Daten, welche eher stark von der Normalverteilung abweichen. Für einen Datensatz der nach Marktkapitalisierung 10\% kleinsten und größten Unternehmen der CRSP Datenbank stellt Patton (2004) fest, dass die Berücksichtigung von Koschie-

8 Der untersuchte Modellrahmen in Hong, Tu und Zhou (2007) ist wie in Kandel und Stambaugh (1996) und Pástor und Stambaugh (2000) gewählt.

9 Weitere Studien, welche auf einen bedeutenden Nutzenverlust hinweisen, sind unter anderem Adler und Kritzman (2007), Cvitanic, Polimenis und Zapatero (2008) und Sharpe (2007).

10 Vgl. auch Goetzmann und Kumar (2008). 
fen über geeignete Copulas out-of-sample von Vorteil ist. Die Ergebnisse werden jedoch bei Berücksichtigung von Leerverkaufsbeschränkungen statistisch nicht mehr signifikant. Für den Aktienmarkt untersuchen insbesondere Martellini und Ziemann (2010) die Vorteilhaftigkeit von höheren Momenten in der Asset Allokation auf Basis eines erweiterten Minimum-Varianz-Ansatzes, der auch Präferenzen für Schiefe und Kurtosis berücksichtigt. Für einen monatlichen Anlagehorizont wird eine Verschlechterung des out-of-sample Nutzens gegenüber dem globalen Minimum-Varianz-Portfolio ermittelt. Lediglich bei wöchentlichen und täglichen Horizonten, für die die Renditezeitreihen tendenziell eher von der Normalverteilung abweichen, konnte eine Verbesserung erzielt werden.

Die Ergebnisse der out-of-sample Studien können keinen eindeutigen Vorteil aus der Berücksichtigung der höheren Momente in der Asset Allokation feststellen. Die exaktere Erfassung der Präferenzen des Investors wird daher durch die Güte der Erwartungsbildung überlagert und wirft unmittelbar die Frage nach geeigneten Schätzverfahren für die Verteilung der zukünftigen Renditen und deren Momente auf.

\subsection{Historische Schätzverfahren und der Umgang mit Schätzfehlern}

Die für die Asset Allokation benötigten Erwartungen über die zukünftige Entwicklung der zur Verfügung stehenden Anlagewerte können mit Hilfe von Schätzverfahren aus historischen Renditezeitreihen bestimmt werden. Diese unterliegen jedoch zwei konzeptionellen Problemen in der Anwendung. Zum einen ergeben sich bei der Verwendung statistischer Schätzer Schätzfehler. Dies ist darin begründet, dass die Schätzer wiederum selbst Zufallsvariablen sind und somit die Realisationen um den "wahren" Wert streuen. Aufgrund des Gesetzes der großen Zahlen konvergieren bei hinreichend langen Schätzzeiträumen diese Schätzer zwar gegen den "wahren" Parameterwert, jedoch ist einerseits nicht gewährleistet, dass eine hinreichende Länge der Zeitreihen existiert, andererseits ist fragwürdig, inwieweit historische Renditen, von vor z. B. 50 Jahren, eine gültige Aussagekraft für aktuelle Parameterwerte haben. Dies führt unmittelbar zu der zweiten konzeptionellen Schwäche von Schätzverfahren mittels historischer Zeitreihen, nämlich der Stationaritätsannahme. Diese besagt, dass der zukünftige Kurs eines Anlagewertes die Realisation aus der historisch geschätzten Wahrscheinlichkeitsverteilung ist. Diese Annahme ist jedoch nur bedingt haltbar, da Strukturbrüche, wie Schocks in Form von Kurssprüngen oder Volatilitätsanpassungen, unmittelbar Preise sowie die gesamte Struktur der Wahrscheinlichkeitsverteilung beeinflussen. 
Vor dem Hintergrund der beschriebenen Probleme bei Verwendung historischer Zeitreihen in der Asset Allokation werden in der Forschung verschiedene Ansätze betrachtet, um insbesondere den Einfluss von Schätzfehlern auf die "optimale" Vermögensverteilung zu reduzieren. ${ }^{11}$ Auf die Stationaritätsannahme bei historischen Zeitreihen kann hingegen nicht verzichten werden. Zwar können beispielsweise durch Markov Switching Modelle für verschiedene Zustände unterschiedliche Wahrscheinlichkeitsverteilungen modelliert werden, diese müssen jedoch in den jeweiligen Zuständen weiterhin als stationär angenommen werden. Zur Begrenzung von Schätzfehlern werden insbesondere die Verwendung bayesianischer Methoden und die Anwendung von Shrinkage Ansätzen diskutiert.

Als Beispiele bayesianischer Methoden in der Asset Allokation seien folgende zentrale Arbeiten genannt: ${ }^{12}$ Jorion (1986) verwendet anstatt des Stichprobenschätzers ${ }^{13}$ einen Bayes-Stein Schätzer für den Erwartungswert. Konkret wird bei diesem Ansatz der Erwartungswert eines jeden riskanten Anlagewertes a-priori gleich dem Erwartunsgwert des globalen Minimum-Varianz-Portfolios gesetzt. ${ }^{14,15}$ Pástor (2000) und Pástor und Stambaugh (2000) untersuchen die Verwendung von Faktor-Strukturen zur Beschreibung von Assetrenditen in der Portfolioselektion, indem sie a-priori ein Faktor-Modell für die Entwicklung der riskanten Anlagewerte unterstellen. Das spezifische Modell ist dabei flexibel, typische Anwendungen sind das Capital Asset Pricing Modell, das Drei-Faktor-Modell nach Fama und French (1993) und das Vier-Faktor-Modell nach Carhart (1997). Der Faktor bzw. die Faktoren selbst können dabei als unbeobachtet angenommen werden. Für die abgeleitete Verbindung zwischen Kovarianzmatrix und Erwartungswert zeigen MacKinlay und Pástor (2000) insbesondere für den Erwartungswert stabilere und exaktere Schätzwerte sowie Nutzenvorteile in der Portfolioselektion. ${ }^{16}$

Den zweiten Ansatz zur Reduktion von Schätzfehlern stellen Shrinkage Verfahren dar, welche auf der Annahme spezifischer Abhängigkeitsstrukturen unter den Assets basieren.

11 Für eine Einführung in die Portfoliotheorie mit speziellem Fokus auf Parameterunsicherheiten vgl. Brandt (2010).

12 Für einen Literaturüberblick zur Verwendung bayesianischer Verfahren in der Portfolioselektion siehe Avramov und Zhou (2010).

13 Insbesondere der Erwartungswert gilt als schwer zu schätzen (vgl. z. B. Merton (1980), Best und Grauer (1991) und Chopra und Ziemba (1993)).

14 Der Erwartungswert des globalen Minimum-Varianz-Portfolios wird aus dem Stichprobenschätzer und den Gewichten des globalen Minimum-Varianz-Portfolios, welche auf der Schätzung der Kovarianzmatrix basieren, bestimmt.

15 In diesem Zusammenhang zeigen Kan und Zhou (2007) und Siegel und Woodgate (2007), dass bei expliziter Modellierung des Schätzfehlers das Tangentialportfolio nicht zwangsweise optimal ist. Um dieser Problematik zu begegnen, wird vorgeschlagen, anstatt ausschließlich eine Mischung aus risikofreier Anlage und Tangentialportfolio zu wählen, zusätzlich das globale Minimum-VarianzPortfolio in der optimalen Asset Allokation zu berücksichtigen. 
Grundprinzip dieses Ansatzes ist die Mischung aus zwei unterschiedlichen Schätzverfahren für die Bestimmung der unbekannten Parameter, typischerweise zwischen einem strukturierten (basierend auf einer spezifischen Abhängigkeitsstruktur) und dem entsprechenden unstrukturierten Stichprobenschätzer. Beispielhaft seien hier die Ansätze von Ledoit und Wolf (2003) und Ledoit und Wolf (2004) vorgestellt. Die Autoren bestimmen optimale Mischungen aus Schätzern für die Kovarianzmatrix, wobei einerseits eine Mischung aus dem Stichprobenschätzer und dem Schätzer bei Annahme einer konstanten Korrelation zwischen den Assets nach Elton und Gruber (1973), andererseits eine Mischung aus dem Stichprobenschätzer und dem Schätzer bei Annahme eines Ein-Faktor-Modells nach Sharpe (1963) hergeleitet wird. Die Verwendung strukturierter Schätzer bzw. Schätzer aus einem Shrinkage Ansatz ermöglicht gegenüber dem unstruktierten Stichprobenschätzer große Vorteile in der Asset Allokation und verringert Schätzfehler sowie Transaktionskosten. Letzters ist darin begründet, dass durch die Strukturierung weniger extreme Gewichte in der resultierenden optimalen Vermögensverteilung gewählt werden.

Anstatt sich mit weiteren Schätzmethodiken für die benötigten Parameter zu befassen, konzentriert sich ein neuerer Literaturstrang auf die direkte Betrachtung der optimalen Gewichtung der Anlagewerte. In diesem Zusammenhang wird insbesondere die Wirkung von Restriktionen auf die Gewichte der einzelnen Anlagewerte diskutiert. Jagannathan und Ma (2003) zeigen beispielhaft, dass eine strikte Leerverkaufsbeschränkung einen signifikant positiven Einfluss auf die out-of-sample Performance hat. ${ }^{17}$ In einem allgemeineren Ansatz zur Begrenzung von Portfoliogewichten leiten DeMiguel, Garlappi, Nogales und Uppal (2009a) analytisch her, dass Restriktionen zum einen eine strukturierende Wirkung auf die Schätzer haben und zum anderen eine bayesianische Interpretation besitzen. Unter anderem wird der zuvor diskutierte Shrinkage Ansatz von Ledoit und Wolf (2004) bei spezifischen Annahmen an die Gewichte als Spezialfall bestimmt und es wird gezeigt, dass die Restriktionen einer zuvor definierten a-priori Gewichtung entsprechen. In einer empirischen Studie wird die Vorteilhaftigkeit von Restriktionen auf die Gewichte wie in Jagannathan und Ma (2003) bestätigt.

Einen fundamental anderen Ansatz erarbeiten Brandt, Santa-Clara und Valkanov (2009): die sogenannten "parametrischen Portfolios". Anstatt die Gewichte aus einem Optimierungsprozess zu erhalten, werden die Gewichte selbst als Funktionen modelliert und in Abhängigkeit von Charakteristika der einzelnen Assets ausgedrückt. In ihrer Studie nutzen die Autoren die Parameter aus dem Drei-Faktor-Modell nach Fama und French (1993) und stellen fest, dass die hergeleitete Methodik auch out-of-sample enorme Potentiale für die Asset Allokation birgt.

17 Vgl. auch Frost und Savarino (1988). 
Im Rahmen der Portfolioselektion mit höheren Momenten und der Berücksichtigung von Schätzfehlern seien zwei Studien vorgestellt. Zum einen verfolgen Harvey, Liechty, Liechty und Muller (2010) einen bayesianisches Verfahren und nutzen zur Modellierung der Renditeverteilung einen parametrischen Ansatz auf Basis der Schiefen-Normalverteilung. Diese Verteilung erlaubt es, asymmetrische Dichten und Fat-Tails in der Dichte zu modellieren und somit in der optimalen Asset Allokation zu berücksichtigen. Zum anderen erweitern Martellini und Ziemann (2010) die Shrinkage Ansätze von Ledoit und Wolf (2003) und Ledoit und Wolf (2004) für den Fall höherer Momente. Sie zeigen, dass sich die Vorteilhaftigkeit bei Verwendung strukturierter Schätzer auch im Falle von höheren Momenten bestätigt.

Die Berücksichtigung von Schätzfehlern, entweder durch bayesianische oder Shrinkage Änsatze wie auch durch die Restriktionen der Gewichte im Portfolio, besitzt out-of-sample große Vorteile gegenüber unstrukturierten Stichprobenschätzern. Dennoch zeigen DeMiguel, Garlappi und Uppal (2009b) in einer umfangreichen empirischen Studie, dass keine der vorgestellten aktiven Portfoliostrategien out-of-sample gegenüber einer passiven naiven Anlage, welche das Vermögen gleichmäßig auf die zur Verfügung stehenden Anlagewerte verteilt, eine signifikant bessere Performance liefert. ${ }^{18}$ Auf Grundlage dieser Erkenntnis verbinden Tu und Zhou (2011) aktive Strategien, wie das Tangentialportfolio nach Markowitz (1952), die bayesianischen Ansätze nach Jorion (1986) und MacKinlay und Pástor (2000) sowie den Ansatz von Kan und Zhou (2007) mit der naiven Anlage und zeigen eine Verbesserung der out-of-sample Performance gegenüber der naiven und der jeweiligen aktiven Strategie. Die gemischten Portfolios können dabei aus theoretischer Sicht als Shrinkage Ansätze kategorisiert werden.

Die vorgestellten Portfoliostrategien verwenden allesamt historische Renditezeitreihen zur Bestimmung der optimalen Vermögensaufteilung und unterliegen somit den zuvor diskutierten zentralen Problemen wie Schätzfehlern und Stationaritätsannahmen. In dieser Arbeit soll nun ein fundamental anderer Ansatz zur Bildung der Erwartungen für die zukünftige Entwicklung der Anlagewerte verfolgt werden. Hierzu sollen Informationen aus dem Derivatemarkt, welche implizit in den Preisen von Optionen enthalten sind, extrahiert und zur Erwartungsbildung genutzt werden sowie anschließend in der Asset Allokation mit höheren Momenten Anwendung finden.

$18 \quad$ Vgl. auch Michaud (1989). 


\title{
1.3 Implizite Informationen in Optionsmarktdaten
}

\author{
"The development of option-pricing theory \\ is perhaps the most significant achievement in financial economics, \\ if not all of economics." \\ (Mark Kritzman in Jackwerth (2004))
}

Die Beiträge von Black und Scholes (1973) und Merton (1973), die zu dem bekannten Black-Scholes-Merton Optionspreismodell führen, können als zwei der bedeutendsten Arbeiten der modernen Finanzwirtschaft gewertet werden. Insbesondere die teilweise Möglichkeit zur Formulierung geschlossener Formeln zur Bewertung von Derivaten, wie der Black-Scholes Formel für europäische Optionen, hat ohne Zweifel die Bedeutung und Anwendung von Derivaten in den internationalen Finanzmärkten intensiviert.

Aus der Tatsache, dass die Auszahlung eines Derivats bei Verfall oder bei vorzeitiger Ausübung auf der Entwicklung des zugrundeliegenden Basiswertes basiert, lässt sich eine natürliche Verbindung zwischen Kassa- und Derivatemarkt ableiten. Beispielhaft sei hier an eine europäische Call-Option auf eine Aktie gedacht, deren Ausübungspreis mit dem heutigen Kurs übereinstimmt. Das Derivat beinhaltet das Recht, am Ende der Laufzeit die Aktie zum heutigen Kurs zu kaufen, oder, falls der Kurs unterhalb des Ausübungspreises liegt, die Option verfallen zu lassen. Der Wert der Option steht somit bei Verfall unmittelbar mit dem zukünftigen Kurs in Beziehung. Informationen, welche nun aus dem heutigen Optionspreis am Derivatemarkt gewonnen werden können, haben infolgedessen ein großes Potential zur Beschreibung der erwarteten Entwicklung des zugrundeliegenden Basiswertes. Das Konzept dieser impliziten Informationen ist grundsätzlich zukunftsorientiert und unterliegt daher nicht der Problematik einer Stationaritätsannahme.

Das bekannteste und bedeutendste Beispiel einer solchen impliziten Information ist die implizite Volatilität. Einer der ersten Beiträge zur Untersuchung dieser Größe, welche durch Inversion der Black-Scholes Formel nach dem Volatilitätsparameter aus dem Preis einer Option gewonnen werden kann, stammt von Latané und Rendleman (1976). Die Autoren zeigen im Rahmen einer empirischen Studie, dass die implizite Volatilität eine genauere Vorhersagekraft für die zukünftige Volatilität des Basiswertes hat als Prognosen, die aus der Auswertung von historischen Renditezeitreihen hervorgehen. Ausgehend von dieser Erkenntnis hat sich ein Literaturstrang entwickelt, der die Vorteilhaftigkeit von impliziten Informationen gegenüber historischen Verfahren testet. ${ }^{19}$

19 Für einen umfassenden Literaturüberlick zur Verwendung impliziter Informationen in Prognosemodellen siehe Chang, Christoffersen und Jacobs (2011b). 
Historisch betrachtet lag der Fokus der Forschung zur Verwendung impliziter Informationen überwiegend auf der implizit bestimmten Volatilität. Dies ist darin begründet, dass eine stabile Bestimmung impliziter Informationen ausschließlich mit Hilfe von spezifischen Optionspreismodellen möglich war und Modelle, die auf andere Parameter abzielen, nicht etabliert waren. ${ }^{20}$

Die ursprüngliche Beobachtung von Latané und Rendleman (1976) konnte in einer Vielzahl von Studien bestätigt werden. ${ }^{21}$ Obschon die Güte der Prognose durch modellbasierte implizite Volatilitäten gegenüber historischen Verfahren verbessert werden kann, ist die tatsächliche Prognosequalität schwer zu erfassen, da stets zwei Dinge simultan getestet werden: Neben der Prognosequalität des impliziten Schätzwertes wird immer auch gleichzeitig das spezifische Optionspreismodell geprüft, aus welchem der Parameter gewonnen wurde. Um dieser Problematik zu begegnen wurde der Ansatz verfolgt, die implizite Volatilität unabhängig von einem spezifischen Modell darzustellen. Britten-Jones und Neuberger (2000) leiten in diesem Zusammenhang aus dem Konzept der vollständig modellfrei bestimmten Dichte nach Breeden und Litzenberger (1978) eine modellfreie Entsprechung der impliziten Volatilität her. ${ }^{22}$ Die hergeleitete Methodik ist auch für die Praxis von großer Bedeutung, da sie die Grundlage für Volatilitätsindices wie den VIX der CBOE oder den VDAX-NEW der Deutschen Börse darstellt. Die Prognosequalität der modellfreien impliziten Volatiltät wurde in Jiang und Tian (2005) bestätigt. Darüber hinaus wurde dargelegt, dass diese auch implizite Volatilitäten aus dem Black-Scholes Modell dominiert. ${ }^{23}$

20 Vollständig modellfreie Ansätze, wie die Dichtebestimmung nach Breeden und Litzenberger (1978), waren zwar verfügbar, lieferten jedoch instabile Schätzungen oder waren mangels der benötigten quantitativen Menge an Derivaten gar nicht durchführbar.

21 Vgl. hierzu den Literaturüberblick in Poon und Granger (2003). Einen höheren Prognosegehalt der impliziten Volatilität, bestimmt auf Basis spezifischer Optionspreismodelle, gegenüber historischen Schätzverfahren für die realisierte Volatilität zeigen unter anderem Beckers (1981), Day und Lewis (1992), Lamoureux und Lastrapes (1993), Jorion (1995), Fleming (1998) und Ederington und Guan (2002). Eine schlechtere Prognosequalität finden Canina und Figlewski (1993). Martens und Zein (2004) zeigen, dass aus Intraday-Daten bestimmte Momente eine vergleichbare und teilweise verbesserte Prognosequalität besitzen.

22 Die implizite Volatilität nach Britten-Jones und Neuberger (2000) hat neben der Eigenschaft, ohne ein spezifisches Optionspreismodell bestimmt zu werden, auch den Vorteil, nicht dem Problem einer instabilen Schätzung der Dichte zu unterliegen.

23 Vgl. auch Blair, Poon und Taylor (2001), Swidler und Wilcox (2002). Busch, Christensen und Ørregaard Nielsen (2011) zeigen, dass die implizite Volatilität auch im Rahmen fortgeschrittener ökonometrischer Verfahren auf Basis historischer Renditezeitreihen zur Prognose realisierter Volatilitäten für Währungen, Aktien und Anleihen hohen Erklärungsgehalt hat. 
Theoretisch betrachtet entsprechen implizite Informationen der risikoneutralen Dichte bzw. deren Momenten. ${ }^{24}$ Mit Hilfe des Spanning-Arguments aus Carr und Madan (2001) leiten Bakshi, Kapadia und Madan (2003) die risikoneutralen Momente der Log-Renditeverteilung des zugrundeliegenden Basiswertes modellfrei her. Hieraus können somit auch die höheren Momente wie Schiefe und Kurtosis implizit bestimmt werden. Diese modellfreien impliziten Momente stellen die Grundlage für eine Vielzahl von weiteren Studien dar und ermöglichen auch die Identifikation weiterer impliziter Größen. ${ }^{25}$

So leiten Skintzi und Refenes (2005) und Driessen, Maenhout und Vilkov (2009) aus dem Vergleich der impliziten Volatilität einer Index-Option und den impliziten Volatilitäten der einzelnen Mitglieder des Indexes sowie der Annahme einer konstanten Korrelation die implizite Korrelation her. Die Autoren zeigen, dass dieser gewonnene Parameter eine bessere Prognosefähigkeit der zukünftig realisierten Korrelation hat als historische Schätzverfahren. Chang, Christoffersen, Jacobs und Vainberg (2011a), Buss und Vilkov (2012) und Baule, Korn und Saßning (2013) leiten mit Hilfe der Annahme eines Ein-Faktor MarktModells aus Index-Optionen implizite $\beta$-Faktoren für die Mitglieder des Indexes her und zeigen, dass diese implizit gewonnenen Faktoren teilweise einen besseren Prognosegehalt haben als Verfahren auf Basis historischer Renditezeitreihen. Implizite Korrelationen und $\beta$-Faktoren sind auch im Rahmen der Asset Allokation von großer Bedeutung, da sie die Möglichkeit eröffnen, Abhängigkeitsstrukturen unter verschiedenen Anlagewerten zu modellieren.

Im Vergleich zu geschätzten Parameterwerten aus historischen Zeitreihen besitzen implizite Informationen den Vorteil, weder einer Stationaritätsannahme noch Schätzfehlern im Sinne der Stichprobenvariation zu unterliegen. Dies ergibt sich zum einen daraus, dass sie ausschließlich aus einem Querschnitt von Optionspreisen an einem einzigen Stichtag bestimmt werden und somit die ermittelte Wahrscheinlichkeitsdichte bzw. das Moment der risikoneutralen Wahrscheinlichkeitsverteilung ohne Rückgriff auf historische Daten auskommt. Zum anderen müssen die impliziten Informationen nicht "geschätzt" werden, sondern können auf Basis numerischer Verfahren berechnet werden. ${ }^{26}$ Trotzdem haben

24 Für einen Literaturüberblick und eine Gegenüberstellung von Verfahren zur Gewinnung der vollständigen impliziten Dichte auf Basis modellfreier und parametrischer Ansätzen siehe Bliss und Panigirtzoglou (2002) und Jackwerth (2004).

25 Die zeitliche Variation der risikoneutralen Momente nach Bakshi, Kapadia und Madan (2003) untersuchen Konstantinidi, Skiadopoulos und Tzagkaraki (2008) für die Volatilität sowie Neumann und Skiadopoulos (2012) für Schiefe und Kurtosis. Beide Studien kommen zu dem Ergebnis, dass sich die zeitliche Variation der Momente statistisch prognostizieren lässt. Handelsstrategien, welche auf die zeitliche Veränderung der impliziten Momente abzielen, zeigen jedoch bei Berücksichtigung von Transaktionskosten keine signifikanten Excess Returns.

26 Der theoretische Wert der impliziten Information besitzt keine Ungenauigkeiten in der Bestimmung. Bei der praktischen Umsetzung hingegen können eine Vielzahl von Problemen entstehen. Unter anderem werden Optionen nicht zu beliebigen Ausübungspreisen gehandelt und es müssen daher 
implizite Informationen einen entscheidenden Nachteil gegenüber historischen Schätzwerten: Sie werden unter dem risikoneutralen Wahrscheinlichkeitsmaß bestimmt und haben somit aus theoretischer Sicht nur eine bedingte Aussagekraft für Realisationen unter dem physischen bzw. dem "Real-World" Maß. Um dieser Problematik zu begegnen, kann mit Hilfe der Theorie eines repräsentativen Investors ein Übergang zwischen risikoneutralem und physischem Moment erreicht werden. Dieser Prozess wird in der Literatur auch als Risikoadjustierung bezeichnet. Grundlage sind die risikoneutrale und physische Wahrscheinlichkeitsverteilung sowie die Nutzenfunktion des repräsentativen Investors. Dabei kann über die Charakterisierung des repräsentativen Investors stets eine der drei Funktionen aus den anderen beiden abgeleitet werden. ${ }^{27}$ Ein besonderer Fokus der Forschung liegt in diesem Zusammenhang auf der aus einem Vergleich von risikoneutraler und physischer Dichte gewonnenen Nutzenfunktion des repräsentativen Investors. Dabei wurde festgestellt, dass dieser nicht ausschließlich risikoavers ist, sondern auch negative Risikoaversionsparameter annehmen und damit als risikosuchend charakterisiert werden kann (vgl. Jackwerth (2000)). ${ }^{28}$

Die Bedeutung und Notwendigkeit der Risikoadjustierung zeigt ein Vergleich zwischen impliziter und realisierter Volatilität. So wurde insbesondere für Index-Optionen empirisch eine signifikante Überschätzung des realisierten Wertes durch das risikoneutrale implizite Moment beobachtet. Die Differenz aus den Werten wird als Varianz-Risikoprämie bezeichnet und ist von großem Interesse in der Forschung. So zeigen Bakshi und Madan (2006), dass die Varianz-Risikoprämie mit den höheren Momenten des physischen Maßes in Verbindung steht, und Bollerslev, Gibson und Zhou (2011) kommen zu dem Ergebnis, dass die Prämie mit Hilfe von Makro-Finanzdaten erklärt werden kann. Schließlich untersuchen Carr und Wu (2009), wie die Differenz, welche der Auszahlung eines Varianz-Swaps entspricht, mit Hilfe eines Portfolios aus Optionskontrakten realisiert werden kann. In einem vergleichbaren Zusammenhang zeigen Kozhan, Neuberger und Schneider (2013), durch den Vergleich von realisierter und impliziter Schiefe, die Existenz einer SchiefeRisikoprämie.

Weitere empirische Studien leiten einen statistisch signifikanten Zusammenhang zwischen impliziter Information bzw. derer abgeleiteten Größen mit der zukünftigen Rendite des zugrundeliegenden Basiswertes her. So stellen Bollerslev, Tauchen und Zhou (2009) und

hypothetische Preise bestimmt werden (vgl. hierzu Jiang und Tian (2005)). Auch sollte kritisch hinterfragt werden, ob die ursprüngliche preisrelevante Information des Marktteilnehmers, die aus den Optionen gewonnen wird, ohne Schätzfehler eingegangen ist.

27 Vgl. unter anderem Bliss und Panigirtzoglou (2004), Bakshi, Kapadia und Madan (2003) und Liu, Shackleton, Taylor und Xu (2007).

28 Weitere Studien, welche die Form der Nutzenfunktion des repräsentativen Investors untersuchen, sind unter anderem Rosenberg und Engle (2002) und Ziegler (2007). 
Driessen, Maenhout und Vilkov (2012) fest, dass die Varianz-Risikoprämie und die implizite Korrelation erklärenden Gehalt für die zukünftige Indexrendite hat. Im Rahmen des Querschnitts konnten auf Basis von Sorting-Portfolios und Fama und MacBeth (1973) - Regressionen für die implizite Volatilität, Schiefe und Kurtosis signifikante Zusammenhänge zwischen impliziten Momenten und zukünftiger Rendite bestimmt werden. ${ }^{29}$ Vilkov und Xiao (2012) zeigen, dass das aus der Extremwerttheorie implizit abgeleitete Tail Loss Measure insbesondere zur Vorhersage von negativen Renditen und Crashes genutzt werden kann. ${ }^{30}$ Duan und Zhang (2010) und Karoui (2011) leiten aus theoretischen Modellen eine implizite Marktrisikoprämie durch Vergleich physischer und risikoneutraler Momente her und zeigen, dass diese auch empirisch im Vergleich zu etablierten Verfahren wie in Goyal und Welch (2008) einen bedeutenden Erklärungsgehalt für die zukünftige realisierte Überrendite hat.

Zusammenfassend liefern implizite Informationen enorme Potentiale zur Beschreibung der zukünftigen Entwicklung des zugrundeliegenden Basiswertes. Neben der impliziten Volatilität, die insbesondere zur Erklärung der realisierten Volatilität herangezogen werden kann, wurden auch die höheren Momente wie implizite Schiefe und Kurtosis erfolgreich zur Beschreibung der zukünftigen Entwicklung identifiziert. Die Verwendung und Betrachtung von impliziten Informationen in der Asset Allokation ist daher ein relevanter Forschungsgegenstand, welcher in dieser Arbeit verfolgt werden soll.

\subsection{Forschungsfragen und Gang der Untersuchung}

Aus den einführenden Bemerkungen lassen sich zwei zentrale Forschungsfragen für die empirischen Studien dieser Arbeit ableiten.

29 Vgl. Conrad, Dittmar und Ghysel (2013), Rehman und Vilkov (2012), Chang, Christoffersen und Jacobs (2013), Bali und Murray (2013), Bali und Hovakimian (2009) und Xing, Zhang und Zhao (2010).

30 Bezüglich des Zusammenhangs von impliziter Information und Rendite zeigen Bakshi, Panayotov und Skoulakis (2011), dass die Änderung der risikoneutralen integrierten Varianz (Forward Variance) erklärenden Gehalt für das Wachstum von realwirtschaftlicher Aktivität, für Zinssätze, für Aktienmarktrenditen und für Änderungen in der Varianz-Swap-Rate hat. Cremers und Weinbaum (2010) finden, dass bei Abweichung von der Put-Call Parität, gemessen als Differenz in der impliziten Volatilität von Call und Put Optionen, Assets mit relativ zu teuren Calls diejenigen mit relativ zu teuren Puts outperformen. Agarwal, Bakshi und Huij (2009) zeigen, dass die Änderungen von impliziten Momenten Hedge-Fund Returns erklären. Diavatopoulos, Doran, Fodor und Peterson (2012) untersuchen Aktienkursbewegungen nach Bekanntgabe von Geschäftszahlen und zeigen, dass die implizite Schiefe und Kurtosis einen bedeutenden Erklärungsgehalt haben. 
(i) Ist die Verwendung von impliziten Informationen in der Asset Allokation mit höheren Momenten in einem out-of-sample Setting vorteilhaft gegenüber passiven und aktiven Anlagestrategien auf Basis historischer Renditezeitreihen?

(ii) Welchen Einfluss hat die Berücksichtigung der höheren impliziten Momente Schiefe und Kurtosis in der Asset Allokation verglichen mit derjenigen, die ausschließlich auf Erwartungswert und Varianz basiert?

Für die empirischen Studien müssen die Forschungsfragen differenziert nach den zur Verfügung stehenden Anlagewerten und dem betrachteten Investor untersucht werden. Hierzu kann das Investmentproblem in die Bereiche Market-Timing, strategische und taktische Asset Allokation unterteilt werden. Darüber hinaus wird berücksichtigt, ob der Investor seine Investitionsentscheidung auschließlich auf Basis der ersten zwei Momente trifft oder höhere Momente in seinem Nutzenkalkül miteinschließt.

Das Market-Timing untersucht die Verteilung des Vermögens auf zwei Anlagewerte: ein riskantes Asset, typischerweise ein Aktienindex, und eine risikofreie Anlage. Die Verwendung von höheren Momenten bzw. der vollständigen Nutzenfunktion in der Asset Allokation mit impliziten Informationen ist in den Studien von Vilkov und Xiao (2012), Kostakis, Panigirtzoglou und Skiadopoulos (2011) und Zdorovenin und Pezier (2011) betrachtet worden. Vilkov und Xiao (2012) zeigen, dass die Verwendung impliziter Informationen, wie das Tail Loss Measure, die implizite Volatilität und Korrelation sowie die VarianzRisikoprämie, große Potentiale für die out-of-sample Performance besitzen. Die verwendete Methodik basiert auf den parametrischen Portfolios nach Brandt, Santa-Clara und Valkanov (2009) und modelliert das Gewicht des riskanten Assets als Funktion der impliziten Information. Die Berücksichtigung der impliziten Informationen erfolgt somit nur indirekt mit Hilfe der spezifischen Gewichtsfunktion. Kostakis, Panigirtzoglou und Skiadopoulos (2011) und Zdorovenin und Pezier (2011) hingegen bestimmen modellfrei die vollständige implizite Dichte nach Breeden und Litzenberger (1978) und transformieren diese mit Hilfe der Methodik von Bliss und Panigirtzoglou (2004) in das physische Maß. Die optimale Asset Allokation erfolgt auf Basis einer direkten Maximierung des Erwartungsnutzens. Die Ergebnisse der out-of-sample Studien bestätigen die Vermutung, dass die unter Verwendung der impliziten Dichte optimale Asset Allokation der aus historischen Zeitreihen hervorgehenden überlegen ist.

Wird das Anlageuniversum um weitere riskante Anlageklassen wie Anleihen und Rohstoffe erweitert, wird das Investmentproblem auch als strategische Asset Allokation bezeichnet. Die Untersuchung des out-of-sample Nutzens von impliziten Informationen wurde in diesem Rahmen in der Literatur noch nicht betrachtet und ist Bestandteil dieser Arbeit. Hierzu ist neben der Auswahl weiterer Anlageklassen im Asset-Mix auch eine Erweiterung 
der Methodik aus Kostakis, Panigirtzoglou und Skiadopoulos (2011) und Zdorovenin und Pezier (2011) erforderlich.

Schließlich wird die Asset Allokation innerhalb einer Anlageklasse, auch als taktische Asset Allokation bezeichnet, betrachtet. Bisherige Studien haben im diesem Rahmen ausschließlich $\mu-\sigma$ Entscheider untersucht. Kempf, Korn und Saßning (2012) zeigen auf Basis vollständig implizit bestimmter Minimum-Varianz-Portfolios, dass diese out-of-sample eine signifikant geringere realisierte Volatilität gegenüber historischen und passiven Benchmark Strategien besitzen. DeMiguel, Plyakha, Uppal und Vilkov (2012) konstruieren optimale Portfolios mit Hilfe der parametrischen Portfolios nach Brandt, Santa-Clara und Valkanov (2009) und kommen zu dem Ergebnis, dass die Berücksichtigung der impliziten Volatilität und Schiefe signifikante Vorteile im Sinne der Sharpe-Ratio hat. Eine Berücksichtigung der höheren impliziten Momente für Investoren ohne $\mu-\sigma$ Nutzenfunktion wurde in der Literatur hingegen noch nicht untersucht und wird in dieser Arbeit getestet. Hierzu ist insbesondere auch die Abhängigkeitsstruktur in den höheren Momenten, in Analogie zur impliziten Korrelation im Falle der Varianz, zu modellieren.

Die Arbeit ist in sechs Kapitel gegliedert. Im Anschluss an die Einleitung leitet das folgende Kapitel die Asset Allokation mit höheren Momenten mit Hilfe einer Taylorentwicklung der Nutzenfunktion her und konkretisiert die in der Arbeit betrachteten Investoren. Das dritte Kapitel untersucht die strategische Asset Allokation mit impliziten Verteilungen. Der Übergang vom risikoneutralen zum physischen Maß wird mit der Annahme eines repräsentativen Investors geleistet. Die Anwendung dieses Verfahrens ist für große Anlageuniversen mit einer Vielzahl von riskanten Anlagewerten nicht übertragbar. Kapitel 4 entwickelt daher zunächst das Konzept risikoadjustierter modellfreier impliziter Momente als direkte Erweiterung der Momente von Bakshi, Kapadia und Madan (2003). Es werden Implikationen aus der Risikoadjustierung diskutiert und die Prognosequalität für entsprechende realisierte Momente getestet. Die taktische Asset Allokation wird abschließend in Kapitel 5 betrachtet. Die Arbeit endet mit einer Schlussbetrachtung, einschließlich kritischer Diskussion der zentralen Forschungsfragen, und gibt einen Ausblick für die weitere Forschung zur Verwendung impliziter Informationen in der Asset Allokation. 



\section{Kapitel 2}

\section{Das Problem der Asset Allokation}

\subsection{Optimale Vermögensverteilung bei Berücksichtigung höherer Momente}

Es wird ein Investor betrachtet, der die Möglichkeit besitzt, sein Anfangsvermögen $W_{0}$ in $N$ verschiedene Anlagewerte zu investieren. Mit $R \in \mathbb{R}^{N}$ wird der Zufallsvektor der Assetrenditen und $\omega \in \mathbb{R}^{N}$ der Vektor der Portfoliogewichte bezeichnet. Das zufällige Endvermögen $W$ des Investors nach einer Periode wird gegeben durch

$$
W=\left(1+\omega^{t r} R\right) \cdot W_{0}
$$

Um sein Vermögen optimal auf die Anlagewerte zu verteilen, wird der Investor seinen erwarteten Nutzen aus dem Investment maximieren. Hierzu wird angenommen, dass der Nutzen des Investors durch die Funktion $U$ in Abhängigkeit des Vermögens $W$ beschrieben werden kann. ${ }^{1}$ Der erwartete Nutzen des Investors wird dann gegeben durch $E\{U(W)\}$.

Die optimale Asset Allokation des Investors $\omega^{*}$, determiniert aus der Maximierung seines erwarteten Nutzens, kann zusammen mit (2.1) als folgendes Optimierungsproblem dargestellt werden:

$$
\omega^{*}=\underset{\omega \in \mathbb{R}^{N} \text {, s.t. } \sum_{i=1}^{N} \omega_{i}=1}{\arg \max } E\left\{U\left(\left(1+\omega^{t r} R\right) \cdot W_{0}\right)\right\},
$$

wobei stets beachtet werden muss, dass die Summe der Gewichte gleich eins ist.

$1 \quad$ Aus technischen Gründen wird weiter angenommen, dass die Nutzenfunktion $U$ unendlich oft differenzierbar ist. 
Eine direkte Lösung des Optimierungsproblems (2.2) für mehrere Anlagewerte ist allgemein nicht möglich. Ausnahmen stellen unter anderem $\mu-\sigma$ Entscheider oder spezifische Verteilungsannahmen an die Assetrenditen dar. Um dieser Problematik zu begegnen und ohne dabei viele Eigenschaften der Verteilung der Anlagewerte und deren Abhängigkeitsstruktur zu unterschlagen, wird dem Ansatz einer Taylorentwicklung der Nutzenfunktion des Investors um dem Erwartungswert $E\{W\}$ gefolgt. $^{2}$

Der Nutzen des Investors lässt sich unter den bisherigen Annahmen wie folgt darstellen:

$$
U(W)=\sum_{k=0}^{\infty}\left[\frac{U^{(k)}(E\{W\})}{k !}(W-E\{W\})^{k}\right] .
$$

Nachfolgend wird ausschließlich eine Entwicklung bis zur vierten Ordnung betrachtet, da zum einen die Wirkungen höherer Momente ab der fünften Ordnung keine intuitive Interpretation besitzen ${ }^{3}$ und zum anderen durch die vierte Ordnung in der Regel ein guter Genauigkeitsgrad der Approximation der Nutzenfunktion gewährleistet ist. Es verbleibt

$$
U(W) \approx U(E\{W\})+\sum_{k=1}^{4}\left[\frac{U^{(k)}(E\{W\})}{k !}(W-E\{W\})^{k}\right]
$$

Durch Erwartungswertbildung auf beiden Seiten der Gleichung lässt sich der Erwartungsnutzen durch die höheren Momente von $W$ approximieren.

$$
\begin{aligned}
E\{U(W)\} \approx & U(E\{W\})+\frac{U^{(2)}(E\{W\})}{2} \mu^{(2)} \\
& +\frac{U^{(3)}(E\{W\})}{6} \mu^{(3)}+\frac{U^{(4)}(E\{W\})}{24} \mu^{(4)}
\end{aligned}
$$

wobei $\mu^{(n)}$ das $n$-te zentrale Moment von $W$ bezeichnet:

$$
\mu^{(n)}=E\left\{(W-E\{W\})^{n}\right\} .
$$

Der erwartete Nutzen des Investors lässt sich somit mittels der zentralen Momente der Verteilung des Endvermögens $W$, nämlich durch Erwartungswert, Varianz, Schiefe und Kurtosis, approximativ bestimmen. Aus dieser Darstellung können durch Auswertung der Nutzenfunktion des Investors und deren Ableitungen an der Stelle $E\{W\}$ die Präferenzen für die zentralen Momente von $W$ abgeleitet werden.

2 Vgl. für diesen Ansatz z. B. Jondeau und Rockinger (2006).

3 Vgl. Martellini und Ziemann (2010) und die darin angegebene Literatur. 
Des Weiteren ist aus der Gleichung (2.4) die Berücksichtigung der höheren Momente in der Asset Allokation ersichtlich. Eine entsprechende Taylorentwicklung bis zur zweiten Ordnung beruht ausschließlich auf Erwartungswert und Varianz. Die genauere Approximation der Nutzenfunktion ermöglicht es, die Präferenzen für Schiefe und Kurtosis des Investors in der Asset Allokation zu erfassen und eine verbesserte Approximation der optimalen Vermögensaufteilung zu gewährleisten.

Insgesamt wird durch Anwendung der Taylorentwicklung $(2.3)^{4}$ auf das Investmentproblem (2.2) das Optimierungsproblem des Investors gegeben durch: ${ }^{5}$

$$
\begin{aligned}
\underset{\omega \in \mathbb{R}^{N}, \text { s.t. } \sum_{i=1}^{N} \omega_{i}=1}{\arg \max _{\omega}}[ & U\left(1+\omega^{t r} E\{R\}\right)+\frac{U^{(2)}\left(1+\omega^{t r} E\{R\}\right)}{2} \omega^{t r} M_{2} \omega \\
& +\frac{U^{(3)}\left(1+\omega^{t r} E\{R\}\right)}{6} \omega^{t r} M_{3}(\omega \otimes \omega) \\
& \left.+\frac{U^{(4)}\left(1+\omega^{t r} E\{R\}\right)}{24} \omega^{t r} M_{4}(\omega \otimes \omega \otimes \omega)\right],
\end{aligned}
$$

wobei $M_{2}, M_{3}, M_{4}$ die Matrizen höherer Ordnung gemäß Jondeau und Rockinger (2006) und Martellini und Ziemann (2010) bezeichnen. ${ }^{6}$ Die anschließenden Ausführungen dienen ihrer Erläuterung.

(i) $M_{2}=E\left\{(R-E\{R\})(R-E\{R\})^{\operatorname{tr}}\right\}$

$M_{2}$ entspricht der Kovarianzmatrix und ist Element in der Klasse der $N \times N$ Matrizen. Auf der Hauptdiagonalen befinden sich die Varianzen der einzelnen Assets, auf den übrigen Einträgen die paarweisen Kovarianzen. Die Multiplikation des Gewichtevektors $\omega$ auf beiden Seiten der Matrix liefert die Portfoliovarianz.

(ii) $M_{3}=E\left\{(R-E\{R\})(R-E\{R\})^{\operatorname{tr}} \otimes(R-E\{R\})^{\operatorname{tr}}\right\}$

$M_{3}$ enthält die zentralen dritten Momente sowie die entsprechenden Komomente dritter Ordnung. $M_{3}$ ist ein Element der Klasse der $N \times N^{2}$ Matrizen und wird als Koschiefematrix bezeichnet. Die Matrix kann als Kombination von $N$ aneinander

4 Vgl. im Rahmen der Anwendung von Taylorentwicklungen in der optimalen Portfolioselektion für Nutzenfunktionen aus der HARA Klasse und deren Approximationseigenschaften für die optimalen Portfoliogewichte Garlappi und Skoulakis (2011).

5 Das Anfangsvermögen des Investors $W_{0}$ sei o. B. d. A. gleich 1.

$6 \quad$ Mit $\otimes$ wird das Kronecker-Produkt bezeichnet. 
gereihten $N \times N$ Matrizen verstanden werden bzw. als aufgeklappter dreidimensionaler Würfel. Für die Einträge von $M_{3}$ hat sich daher folgende Notation etabliert:

$$
s_{i j k}=E\left\{\left(R_{i}-\mu_{i}\right)\left(R_{j}-\mu_{j}\right)\left(R_{k}-\mu_{k}\right)\right\}, \quad i, j, k=1, \ldots, N .
$$

So ist beispielhaft für $N=3$

$$
M_{3}=\left[S_{1}\left|S_{2}\right| S_{3}\right] \quad \text { mit } \quad S_{i}=\left[\begin{array}{lll}
s_{i 11} & s_{i 12} & s_{i 13} \\
s_{i 21} & s_{i 22} & s_{i 23} \\
s_{i 31} & s_{i 32} & s_{i 33}
\end{array}\right]
$$

Die Einträge $s_{i i i}$ entsprechen somit der Schiefe der einzelnen Assets. Die Multiplikation des Gewichtevektors $\omega$ von links und Multiplikation von $\omega \otimes \omega$ von rechts an die Matrix liefert die Portfolioschiefe.

(iii) $M_{4}=E\left\{(R-E\{R\})(R-E\{R\})^{\operatorname{tr}} \otimes(R-E\{R\})^{\operatorname{tr}} \otimes(R-E\{R\})^{\operatorname{tr}}\right\}$

$M_{4}$ wird als Kokurtosismatrix bezeichnet und ist ein Element der Klasse der $N \times N^{3}$ Matrizen. Die Einträge gleichen den zentralen vierten Momenten sowie den entsprechenden Komomenten vierter Ordnung. Die Matrix kann als Kombination von $N^{2}$ aneinander gereihten $N \times N$ Matrizen verstanden werden bzw. als aufgeklappter vierdimensionaler Würfel. ${ }^{7}$ Für die Einträge von $M_{4}$ hat sich folgende Notation etabliert

$$
k_{i j k l}=E\left\{\left(R_{i}-\mu_{i}\right)\left(R_{j}-\mu_{j}\right)\left(R_{k}-\mu_{k}\right)\left(R_{l}-\mu_{l}\right)\right\}, \quad i, j, k, l=1, \ldots, N .
$$

So ist beispielhaft für $N=3$

$$
M_{4}=\left[K_{11} K_{12} K_{13}\left|K_{21} K_{22} K_{23}\right| K_{31} K_{32} K_{33}\right] \quad \operatorname{mit} \quad K_{i j}=\left[\begin{array}{lll}
k_{i j 11} & k_{i j 12} & k_{i j 13} \\
k_{i j 21} & k_{i j 22} & k_{i j 23} \\
k_{i j 31} & k_{i j 32} & k_{i j 33}
\end{array}\right]
$$

Die Einträge $k_{i i i i}$ entsprechen somit der Kurtosis der einzelnen Assets. Die Multiplikation des Gewichtevektors $\omega$ von links und Multiplikation von $\omega \otimes \omega \otimes \omega$ von rechts an die Matrix liefert die Portfoliokurtosis.

Zusammenfassend ist mit der eingeführten Systematik die Investitionsentscheidung des Investors mit Nutzenfunktion $U(W)$ unter Berücksichtigung der höheren Momente der

$7 \quad$ Die Interpretation der Matrizen $M_{2}, M_{3}, M_{4}$ als Würfel wird ausführlich in Jondeau, Jurczenko und Rockinger (2010) diskutiert und mit Abbildungen unterstützt. 
Renditeverteilung für ein Anlageuniversum von $N$ Assets beschrieben. Die optimale Asset Allokation wird dabei durch die Lösung des Optimierungsproblems (2.5) gegeben. ${ }^{8}$

\subsection{Konkretisierung der Investoren und deren Handels- möglichkeiten}

Für den weiteren Verlauf der Arbeit müssen für die empirischen Studien die betrachteten Investoren und deren spezifische Nutzenfunktionen präzisiert sowie deren Handelsmöglichkeiten definiert werden. Dieser Abschnitt dient der Darstellung der betrachteten Investoren sowie der Annahmen bzgl. ihrer Handelsmöglichkeiten.

\subsubsection{Spezifikation der betrachteten Investoren}

Im Folgenden werden Investoren mit einem monatlichen Anlagehorizont betrachtet. Diese Annahme ist in vergleichbarer Literatur üblich und stellt keine bedeutende Einschränkung für die Analysen und Ergebnisse dieser Arbeit dar.

Die Nutzen der Investoren werden auf Basis der CRRA Nutzenfunktion mit Risikoaversionsparameter $\gamma$ modelliert. Die spezifische Funktion $U$ in Abhängigkeit des Vermögens $W$ wird dabei gegeben durch:

$$
U(W)=\frac{W^{1-\gamma}-1}{1-\gamma}, \quad \gamma \neq 1
$$

Die Klasse der CRRA Nutzenfunktionen ist charakterisiert durch eine konstante relative Risikoaversion. Formal bedeutet dies, dass

$$
-W \cdot \frac{U^{(2)}(W)}{U^{(1)}(W)}=\gamma, \quad(\text { konstant })
$$

was gleichbedeutend mit einem konstanten Arrow-Pratt-Maß ist. Ökonomisch hat dies im Rahmen der Asset Allokation die Konsequenz, dass die optimale Vermögensverteilung unabhängig von dem ursprünglichen Vermögen $W_{0}$ ist und somit Entscheidungen bzgl. der Asset Allokation unabhängig von $W_{0}$ getroffen werden können. In der finanzwirtschaft-

$8 \quad$ Im Allgemeinen ist es nicht möglich das Optimierungsproblem (2.5) analytisch zu lösen. In den empirischen Studien dieser Arbeit wird daher auf einen nicht linearen Optimierer zurückgegriffen (R-Funktion: nlm). Weiterführende numerische und statistische Verfahren sind simulationsbasierte Methoden und Grid-Suchen (vgl. hierzu Brandt, Goyal, Santa-Clara und Stroud (2005) und Binsbergen und Brandt (2007)). 
lichen Forschung stellt die CRRA Nutzenfunktion im Rahmen von Portfoliostudien eine Standardklasse zur Modellierung von Investoren dar.

Das Optimierungsproblem (2.5) für die optimale Asset Allokation mit höheren Momenten lässt sich für Investoren mit CRRA Nutzenfunktionen wie folgt formulieren:

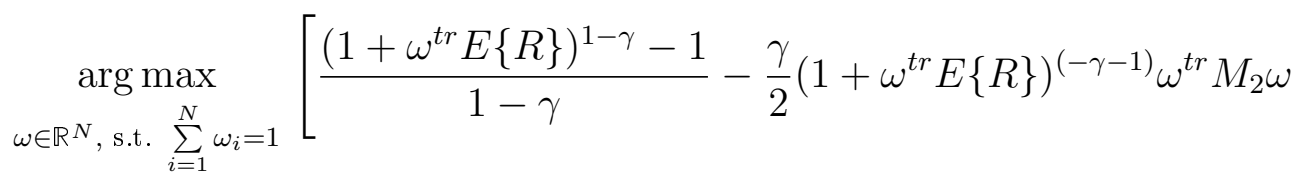

$$
\begin{aligned}
& +\frac{\gamma(\gamma+1)}{6}\left(1+\omega^{t r} E\{R\}\right)^{(-\gamma-2)} \omega^{t r} M_{3}(\omega \otimes \omega) \\
& \left.-\frac{\gamma(\gamma+1)(\gamma+2)}{24}\left(1+\omega^{t r} E\{R\}\right)^{(-\gamma-3)} \omega^{t r} M_{4}(\omega \otimes \omega \otimes \omega)\right] .
\end{aligned}
$$

Für positive $\gamma$ lassen sich unmittelbar die Präferenzen für die Momente der Renditeverteilungen ablesen. So ist die Nutzenfunktion monoton im Erwartungswert steigend ${ }^{9}$ und das Vorzeichen für die Schiefe $\left(M_{3}\right)$ positiv. Der Investor hat somit eine starke Präferenz für einen positiven Erwartungswert sowie für eine positive Schiefe. Für die geraden Momente Varianz $\left(M_{2}\right)$ und Kurtosis $\left(M_{4}\right)$ ist das Vorzeichen negativ und der Investor hat eine negative Präferenz für diese Momente.

Dass die Berücksichtigung der höheren Momente Schiefe und Kurtosis einen bedeutenden Einfluss auf die Vermögensverteilung im Vergleich zu einer Asset Allokation, welche ausschließlich auf Erwartungswert und Varianz basiert, nehmen kann, illustriert das folgende Beispiel. Des Weiteren wird die Konsequenz aus der Vernachlässigung der höheren Momente auf das Nutzenniveau studiert.

\section{Beispiel zum Einfluss höherer Momente auf die Asset Allokation}

Es werden Investoren mit CRRA Nutzenfunktionen und Risikoaversionsparameter $\gamma=$ 2, 4,6 und 8 betrachtet. Das Anfangsvermögen $W_{0}$ der Investoren ist 1. Die Investoren haben jeweils die Möglichkeit, in ein riskantes und in ein risikoloses Asset zu investieren, und bilden somit ein Portfolio aus zwei Anlagewerten. Die Verteilungsparameter der monatlichen Renditen des riskanten Assets $R_{\text {risky }}$ sind wie folgt gewählt worden: ${ }^{10}$

$$
\begin{array}{llllll}
\text { Erwartungswert: } & 10 \% & \text { (annualisiert) } & \text { Volatilität: } & 31 \% & \text { (annualisiert) } \\
\text { Schiefe: } & -0,66 & \text { (standardisiert) } & \text { Kurtosis: } & 5,75 & \text { (standardisiert) }
\end{array}
$$

$9 \quad$ Für $\gamma>0$ und $x>-1$ ist die Funktion: $x \mapsto \frac{(1+x)^{1-\gamma}-1}{1-\gamma}$ strikt monoton steigend.

10 Die Werte sind kalibriert auf monatliche Renditen des S\&P 500 Total Return Index im Zeitraum $1990-2010$. 
Der Zinssatz bzw. die Rendite der risikofreien Anlage $R_{f}$ wird mit $5 \%$ p.a. angenommen. Das zufällige Endvermögen $W$ des Investors wird gegeben durch die Gewichte in der riskanten und risikofreien Anlage $\omega_{\text {risky }}$ und $\omega_{f}$ :

$$
W=1+\omega_{\text {risky }} \cdot R_{\text {risky }}+\omega_{f} \cdot R_{f}
$$

Aus der Bedingung, dass die Summe der Gewichte eins beträgt, wird das Gewicht in der risikofreien Anlage $\omega_{f}$ durch das Gewicht in der riskanten Anlage $\omega_{\text {risky }}$ durch $\omega_{f}=$ $1-\omega_{\text {risky }}$ festgelegt. Die Asset Allokation des Investors wird somit vollständig durch die Wahl des Gewichts $\omega_{\text {risky }}$ beschrieben. Es verbleibt:

$$
W=1+\omega_{\text {risky }} \cdot R_{\text {risky }}+\left(1-\omega_{\text {risky }}\right) \cdot R_{f}
$$

Ausgehend von der spezifischen Risikoaversion $\gamma$ des Investors wird dieser das Gewicht in der riskanten Anlage $\omega_{\text {risky }}$ durch Lösen des Optimierungsproblems (2.7) bestimmen. Hierbei sollte beachtet werden, dass die Varianz und die höheren Momente Schiefe und Kurtosis der Portfoliorendite ausschließlich durch die Verteilung des riskanten Assets determiniert werden.

Um den Einfluss höherer Momente auf das Gewicht der riskanten Anlage und damit auf die optimale Asset Allokation zu untersuchen, werden Schiefe und Kurtosis abwechselnd sowie simultan gleich null gesetzt. Letzterer Fall entspricht somit der Situation, in welcher der Investor seine Asset Allokation nur auf Basis der ersten zwei Momente trifft. Das Gewicht, das aus der Berücksichtigung aller Momente hervorgeht, dient als Benchmark für die Analyse. Tabelle 2.1 zeigt die Ergebnisse.

Tabelle 2.1: Wirkung höherer Momente auf das Gewicht der riskanten Anlage

\begin{tabular}{cccccc}
\hline SKEW & KURT & RRA=2 & RRA=4 & RRA=6 & RRA=8 \\
\hline$-0,66$ & 5,75 & $88,7 \%$ & $44,8 \%$ & $29,9 \%$ & $22,5 \%$ \\
$-0,66$ & 0 & $90,2 \%$ & $45,3 \%$ & $30,2 \%$ & $22,7 \%$ \\
0 & 5,75 & $92,0 \%$ & $46,2 \%$ & $30,9 \%$ & $23,2 \%$ \\
0 & 0 & $93,9 \%$ & $46,8 \%$ & $31,2 \%$ & $23,4 \%$ \\
\hline
\end{tabular}

Diese Tabelle zeigt die Wirkung höherer Momente auf das Gewicht der riskanten Anlage für Investoren mit CRRA Nutzenfunktionen und einem monatlichen Anlagehorizont. Die Verteilungsparameter sind kalibriert auf monatliche Renditen des S\&P 500 Total Return Index im Zeitraum 1990 - 2010 (Erwartungswert 10\% (annualisiert), Volatilität 31\% (annualisiert), Schiefe -0,66 (standardisiert) und Kurtosis 5,75 (standardisiert)). Für die risikofreie Anlage wurde ein Zinssatz von $5 \%$ p.a. angenommen.

Aus dem Vergleich der Gewichte innerhalb der gleichen Verteilungsparametersituation lässt sich zunächst der Einfluss der Risikoaversion auf die optimale Allokation untersuchen. 
Je risikoaverser der Investor ist, desto geringer ist der Anteil der riskanten Anlage bzw. desto höher ist das Gewicht der risikofreien. Das Risiko des Portfolios, ausgedrückt durch Varianz, Schiefe und Kurtosis, wird determiniert durch den Anteil der riskanten Anlage. Sinkt dieser Anteil, verringert sich auch das einzugehende Risiko auf Ebene des Portfolios. Die Risikoaversion des Investors kommt somit unmittelbar in der Portfoliokomposition zum Ausdruck.

Bei Vernachlässigung der Schiefe oder Kurtosis in der optimalen Asset Allokation wird ein erwarteter Effekt festgestellt. Die Investoren haben aufgrund ihrer Präferenzen eine Abneigung für negative Schiefe und positive Kurtosis und somit bewirkt die Vernachlässigung dieser Momente, dass das riskante Asset aus Sicht der Investoren attraktiver wird. Folglich steigt der entsprechende Anteil im Portfolio. Werden sogar beide höheren Momente in der Optimierung ausgeschlossen, steigt der Anteil erneut. Insbesondere ein Vergleich zwischen letzterem und dem Fall, bei dem alle Verteilungsinformationen eingehen, zeigt den Einfluss der höheren Momente auf die optimale Asset Allokation. So divergiert beispielsweise für den Investor mit Risikoaversion 4 die optimale Komposition des Portfolios um 2 Prozentpunkte.

Aus der Vernachlässigung der höheren Momente kann ein Nutzenverlust entstehen. Die folgende Tabelle (2.2) zeigt die Differenz der entsprechenden Erwartungsnutzen.

Tabelle 2.2: Nutzenverlust bei Vernachlässigung der höheren Momente

\begin{tabular}{cccccc}
\hline SKEW & KURT & RRA=2 & RRA=4 & RRA=6 & RRA=8 \\
\hline$-0,66$ & 0 & $-0,56$ & $-0,12$ & $-0,05$ & $-0,03$ \\
0 & 5,75 & $-2,65$ & $-0,98$ & $-0,57$ & $-0,39$ \\
0 & 0 & $-6,46$ & $-1,92$ & $-1,05$ & $-0,71$ \\
\hline
\end{tabular}

Diese Tabelle zeigt den Nutzenunterschied bei Vernachlässigung der höheren Momente für Investoren mit CRRA Nutzenfunktionen und einem monatlichen Anlagehorizont. Die Differenzen sind zur Anschaulichkeit mit $10^{6}$ multipliziert angegeben. Die Verteilungsparameter sind kalibriert auf monatliche Renditen des S\&P 500 Total Return Index im Zeitraum 1990 - 2010 (Erwartungswert 10\% (annualisiert), Volatilität $31 \%$ (annualisiert), Schiefe -0,66 (standardisiert) und Kurtosis 5,75 (standardisiert)). Für die risikofreie Anlage wurde ein Zinssatz von $5 \%$ p.a. angenommen.

Die Ergebnisse zeigen übereinstimmend mit den Abweichungen in den Gewichten aus Tabelle (2.2) für den Fall, dass Schiefe und Kurtosis vernachlässigt werden, den größten Nutzenverlust. Dieser ist dabei quantitativ für den risikofreudigsten Entscheider $(R R A=2)$ am größten und steht im Einklang mit der größten Abweichung zwischen den entsprechenden Gewichten.

Zusammenfassend zeigt das betrachtete Beispiel, dass die Verwendung höherer Momente einen Einfluss auf die resultierende optimale Asset Allokation nimmt und bei Vernachlässigung zu einem Nutzenverlust führt. Jedoch ist zu beachten, dass der Nutzenverlust 
in-sample bestimmt wurde. Inwieweit sich dies in einem out-of-sample Rahmen bestätigt, bei welchem zusätzlich auch die Güte der Erwartungsbildung einfließt, ist unklar und wird Bestandteil der Analysen dieser Arbeit sein.

\subsubsection{Annahmen an die Handelsmöglichkeiten der Investoren}

Nach der obigen Spezifikation der betrachteten Investoren der Arbeit werden folgende restringierende Annahmen an die Handelsmöglichkeiten der Investoren gemacht: ${ }^{11}$

(i) Die Investoren haben nicht die Möglichkeit, während ihres Anlagehorizonts Umschichtungen in ihrem Portfolio vorzunehmen.

(ii) Die Investoren haben nicht die Möglichkeit, Short-Positionen bei ihrer Asset Allokation einzugehen und unterliegen somit einer strikten Leerverkaufsbeschränkung.

Die Einschränkung auf Umschichtungen während des Anlagehorizonts verzichten zu müssen, ist in der Untersuchung der taktischen Asset Allokation üblich, in der strategischen Asset Allokation hingegen nicht. Beispielhaft sei in diesem Zusammenhang an einen Investor gedacht, der einen Anlagehorizont von einem Jahr hat, jedoch unterjährig seine Asset Allokation wählt und anpasst. Die Betrachtung von mehreren Umschichtungszeitpunkten innerhalb des Anlagehorizonts kann dabei zu bedeutenden Unterschieden in der optimalen Asset Allokation führen. ${ }^{12}$ Im Rahmen dieser Arbeit soll jedoch der Fokus ausschließlich auf Investmententscheidungen gelegt werden, bei welchen die (perioden-) spezifische Asset Allokation für den zukünftigen Anlagehorizont einer Buy-And-Hold-Strategie gleichkommt. Kapitel 6 weist in diesem Zusammenhang auf den weiteren Forschungsbedarf hin und zitiert grundlegende Literatur.

Die strikte Leerverkaufsbeschränkung ist auch kritisch zu hinterfragen. Jedoch zeigt die verwandte Literatur ${ }^{13}$ die Vorteilhaftigkeit für die out-of-sample Performance dieser Annahme. Des Weiteren stellt sie ein realistisches und praxisnahes Setting dar, da insbesondere Privatpersonen nicht die Möglichkeit besitzen, Short-Positionen bei ihrer Asset Allokation einzugehen.

11 Die Annahmen an die Handelsmöglichkeiten der Investoren werden auf Grund des Umfanges und der Fokussierung der empirischen Studien getätigt.

12 Vgl. Merton (1969), Merton (1971) oder Brennan, Schwartz und Lagnado (1997). Der Unterschied in der optimalen Asset Allokation aus einer einperiodigen und einer mehrperiodigen Betrachtung wird dabei auch als 'Heding Demand" bezeichnet.

13 Vgl. unter anderem Kempf, Korn und Saßning (2012) und DeMiguel, Plyakha, Uppal und Vilkov (2012). 
Zur Untersuchung und Beurteilung der zentralen Forschungsfragen dieser Arbeit wird im nächsten Kapitel die strategische Asset Allokation mit impliziten Informationen betrachtet. 


\section{Kapitel 3}

\section{Strategische Asset Allokation mit impliziten Verteilungen}

\subsection{Einführung}

Die strategische Asset Allokation befasst sich mit der Verteilung von Vermögen auf unterschiedliche Anlageklassen, traditionell auf Aktien sowie kurzfristige und langfristige Anleihen. Zusätzlich werden in Forschung und Praxis auch weitere Anlageklassen, "wie Rohstoffe, Währungen, Unternehmensanleihen, Derivate, Hedge Funds und illiquide Anlagen (Immobilien, Private Equity,...)", betrachtet (DeJong, Schotman und Werker (2008)). Die strategische Asset Allokation hat große Bedeutung für institutionelle Anleger und Pensionsfonds und ist insbesondere von der taktischen Asset Allokation abzugrenzen, im Rahmen derer die Wahl von Investments innerhalb einer Anlageklasse erfolgt.

Für die strategische Asset Allokation ist die Hinzunahme einer Rohstoffklasse zum traditionellen Anlageuniversum von besonderem Interesse. Dies ist darin begründet, dass Rohstoffe geringe Korrelationen zu Aktien und Anleihen aufweisen ${ }^{1}$ und können somit bedeutende Diversifikationsmöglichkeiten und Nutzenvorteile eröffnen. ${ }^{2}$ Die Vorteile sind in einem out-of-sample Rahmen hingegen nicht gesichert. So zeigen Daskalaki und Skiadopoulos (2011), dass sich für Investoren mit Präferenzen für Schiefe und Kurtosis in-sample Nutzenvorteile durch die Hinzunahme einer Rohstoffklasse ergeben, ${ }^{3}$ out-of-sample jedoch nicht. Eine Ausnahme stellt lediglich die Periode des "Rohstoff-Booms" 2005-2008 dar. Die

1 Vgl. z. B. Gorton und Rouwenhorst (2006) und Daskalaki und Skiadopoulos (2011).

2 Die Diversifikationsmöglichkeiten werden aufgrund der fortschreitenden Integration von Rohstoffund Aktienmärkten auch als strittig angesehen (vgl. Silvennoinen und Thorp (2013)).

3 Vgl. hierzu im Rahmen eines $\mu-\sigma$ Entscheiders Jensen, Johnson und Mercer (2000). 
Ursache für die schwachen out-of-sample Ergebnisse können folglich auf Schätzfehler zurückgeführt werden.

Der Rückgriff auf implizite Informationen ist in der strategischen Asset Allokation im Rahmen einer out-of-sample Studie noch nicht betrachtet worden und soll im Folgenden untersucht werden. Neben dem traditionellen Anlageuniversum, bestehend aus den Anlageklassen Aktien, kurzfristige und langfristige Anleihen, wird auch das um eine Rohstoffklasse erweiterte Universum untersucht und die Nutzenvorteile durch die Hinzunahme reflektiert.

Für die Verwendung impliziter Informationen in der strategischen Asset Allokation existiert kein standardisiertes Modell und eine entsprechende Methodik muss daher zunächst entwickelt werden. Ausgangspunkt hierfür ist die Studie von Kostakis, Panigirtzoglou und Skiadopoulos (2011), welche die Asset Allokation mit impliziten Informationen für ein riskantes und ein risikofreies Asset betrachtet. Die Autoren verwenden zur Extraktion der implizten Informationen die modellfreie risikoneutrale Dichte nach Breeden und Litzenberger (1978) und nehmen für den Übergang zum physischen Maß die Existenz eines repräsentativen Investors an. Die Hinzunahme weiterer riskanter Anlagewerte in das Anlageuniversum kann mit dieser Methodik im Rahmen der optimalen Asset Allokation nicht direkt geleistet werden und muss um die Annahme erweitert werden, dass für jede weitere riskante Anlageklasse ein zusätzlicher repräsentativer Investor existiert. Die Modellierung der benötigten Abhängigkeitsstruktur der riskanten Assets erfolgt mit Hilfe einer Copula und die so konstruierte (implizite) Dichte kann für die Lösung der optimalen Asset Allokation herangezogen werden.

Zur Beurteilung der Performance der Asset Allokation mit impliziten Informationen werden dieser zum einen passive Anlagestrategien, zum anderen aus historischen Renditezeitreihen ermittelte Portfolios, gegenübergestellt. Im Rahmen der passiven Anlagestrategien ist insbesondere die Performance einer gleichen Verteilung des Vermögens auf alle verfügbaren Anlageklassen, kurz 1/N Strategie, von großem Interesse. So zeigen Fugazza, Guidolin und Nicodano (2010) für die strategische Asset Allokation, dass die 1/N Strategie Asset Allokationen auf Basis fortgeschrittener historischer Verfahren, wie bayesianischer und ökonometrischer Methoden, vorzuziehen ist. Als aktive Benchmark Strategien dienen Portfolios, welche auf Basis der Annahme der Schiefen-Normalverteilung aus historischen Renditezeitreihen bestimmt werden. Für die Asset Allokation mit höheren Momenten wird dieser Ansatz insbesondere in Harvey, Liechty, Liechty und Muller (2010) propagiert.

Der Einfluss höherer Momente auf die strategische Asset Allokation wird anhand der optimalen Gewichte in den riskanten Anlagewerten untersucht. So leiten im Rahmen normalverteilter Renditen Jurek und Viceira (2011) für Investoren mit CRRA Nutzenfunktionen 
eine Two-Fund Separation her. ${ }^{4}$ Diese besagt, dass sich der Grad der Risikoaversion des Investors ausschließlich im Gewicht der risikofreien Anlage widerspiegelt und nicht in der Komposition des riskanten Portfolioanteils. Weiterführend hat dies zur Konsequenz, dass für jegliche Investoren dieselbe optimale Asset Allokation in den riskanten Anlagewerten gilt. Ob dieses Ergebnis empirisch bei Berücksichtigung höherer Momente, wie Schiefe und Kurtosis, zu bestätigen ist, soll nachfolgend getestet werden.

Mit der empirischen Studie dieses Kapitels sind insbesondere die Arbeiten von Kostakis, Panigirtzoglou und Skiadopoulos (2011), Zdorovenin und Pezier (2011) und Aït-Sahalia und Brandt (2008) verwandt. Erstere studieren die optimale Asset Allokation bzw. das Market Timing mit impliziten Informationen für ein Anlageuniversum bestehend aus einer riskanten und einer risikofreien Anlage. In ihrer empirischen Studie für den S\&P 500 zeigen die Autoren auf Basis einer direkten Maximierung des Erwartungsnutzens, dass Portfoliostrategien mit impliziten Informationen solchen auf Basis historischer Renditezeitreihen out-of-sample überlegen sind. Als Grund für die bessere out-of-sample Performance identifizieren sie eine verbesserte Schätzung des Erwartungswertes.

Zdorovenin und Pezier (2011) untersuchen dasselbe Setting wie Kostakis, Panigirtzoglou und Skiadopoulos (2011) und kommen zu identischen zentralen Ergebnissen. Ein besonderes Augenmerk liegt auf unterschiedlichen Verfahren zur Extraktion der risikoneutralen Dichte. Es wird gezeigt, dass Methoden, die auf statistischer Entropie basieren, solchen Verfahren vorzuziehen sind, die numerisch geglättete Volatilitätsoberflächen heran ziehen.

Aït-Sahalia und Brandt (2008) wiederum untersuchen Konsumentscheidungen und Portfolioselektionen für die strategische Asset Allokation mit risikoneutralen Zustandspreisen. Für die Abhängigkeitsstruktur unter den riskanten Assets (Aktien und Anleihen) wird eine Plackett Copula gewählt. Der Fokus der Studie liegt jedoch nicht auf der out-of-sample Performance von Portfoliostrategien. Vielmehr wird hervorgehoben, dass Konsumentscheidungen auf Basis impliziter und historischer Informationen stark variieren.

Der übrige Teil des Kapitels ist wie folgt gegliedert. Zunächst werden die Daten der empirischen Studie vorgestellt. Es folgt eine ausführliche Beschreibung der verwendeten Methodik und eine Darstellung von Grundlagen zur Extraktion und Risikoadjustierung impliziter Verteilungen sowie zur Konstruktion multidimensionaler Verteilungen. Der darauffolgende Abschnitt stellt das Vorgehen und die Ergebnisse der empirischen Studie vor. Das Kapitel schließt mit einer Zusammenfassung der Resultate.

4 Diese ist auch als Tobin Separation bekannt. 


\subsection{Daten}

Die Untersuchung der strategischen Asset Allokation für Investoren mit einem monatlichen Anlagehorizont ${ }^{5}$ erfolgt anhand von vier alternativen Anlageklassen. Das Assetuniversum umfasst eine risikofreie Anlage, einen Aktienindex, eine langfristige Anleihe und einen Rohstoffindex. Die Repräsentanten der entsprechenden Anlageklassen sind wie folgt gewählt worden.

(i) Risikofreie Anlage: Zinssatz der IvyDB

Für die Investition in eine risikofreie Anlage über den Anlagehorizont wird der durch die Datenbank IvyDB von Optionmetrics bereitgestellte Zinssatz $r$ (p.a.) verwendet. Der Wert eines entsprechenden Investments wird durch $\exp \left(-r \cdot \frac{1}{12}\right)$ bestimmt. $^{6}$

(ii) Aktienindex: S\&P 500 Total Return Index

Als Repräsentant für den Aktienmarkt wird der S\&P 500 Total Return Index gewählt. Der Index berücksichtigt Dividenden und Aktiensplits.

(iii) Langfristige Anleihe: TNX Index

Zur Bestimmung des Preises einer langfristigen Anleihe wird der TNX Index (Ticker: TNX) herangezogen. Dieser gibt die (aktuelle) Yield-To-Maturity einer US Treasury Note mit einer Laufzeit von 10 Jahren wieder. ${ }^{7}$

Der Wert der langfristigen Anleihe wird bestimmt, indem aus dem Kurs des Index $\mathrm{TNX}_{t}$ der Wert eines Zero-Kupon Bonds mit einer Laufzeit von 10 Jahren durch

$$
\exp \left(-10 \cdot \mathrm{TNX}_{t}\right)
$$

gebildet wird. Falls nicht ausdrücklich anders erwähnt, sei im Folgenden mit der Bezeichnung TNX Index die Kurse der so konstruierten Zero-Kupon Bonds gemeint.

(iv) Rohstoffindex: XAU Index

Als Repräsentant für die Investition in die Rohstoffklasse wird der PHLX Gold Silber Sektor Index (Ticker: XAU) gewählt. Der XAU ist ein Aktienindex, welcher die Performance von Gold- und Silber-Minenbetreibern nachbildet. Der XAU Index ist ein Total Return Index und berücksichtigt Dividenden und Aktiensplits.

$5 \quad$ Vgl. die Spezifikation der betrachteten Investoren in Kapitel 2.

$6 \quad$ Der Zinssatz $r$ wird aus der Zinsstruktur abgeleitet, welche aus dem BBA Libor Zinssatz und den Settlement Preisen von CME Eurodollar Futures bestimmt wird (vgl. Optionmetrics Handbuch).

7 Der TNX Index wird auf Basis der Yield-To-Maturity der aktuellsten US Treasury Notes mit einer Laufzeit von 10 Jahren bestimmt. 
Für die empirische Studie wird die Erwartungsbildung der zukünftigen Renditen, welche die optimale Asset Allokation determiniert, anhand von impliziten Informationen aus Optionsmarktdaten und historischen Renditezeitreihen erfolgen. Für die entsprechenden Methodiken müssen verschiedene Daten verfügbar sein.

Die Optionsmarktdaten liegen in der Datenbank IvyDB von Optionmetrics vor und beziehen sich auf Optionen, die an der CBOE gehandelt wurden. Für die Studie werden insbesondere die zur Verfügung gestellten Volatilitätsoberflächen herangezogen. Im Folgenden wird als Stichtag für jeden Monat (Beobachtungszeitpunkt) der erste Montag nach dem dritten Freitag verwendet. Dies entspricht dem ersten Handelstag nach dem Verfallstermin von Optionen an der CBOE und garantiert eine möglichst gute Approximation der Laufzeit der Optionen von einem Monat. Historische Renditezeitreihen werden aus den bereitgestellten Schlusskursen der IvyDB bestimmt. Die Datenbank umfasst den Zeitraum Januar 1996 bis Oktober 2010. Fehlende (historische) Kurse vor 1996 wurden aus Datastream ergänzt. Aufgrund von in-sample Schätzungen beträgt der out-of-sample Zeitraum der empirischen Studie Februar 1999 bis Oktober 2010 und entspricht insgesamt 141 Beobachtungen.

Für den S\&P 500 Total Return Index liegen in der Datenbank keine gehandelten Optionen vor. Zur Bestimmung der impliziten Informationen wird daher auf den S\&P 500 Index zurückgegriffen. Die Anpassung erfolgt, indem der implizit geschätzte Erwartungswert um eine konstante Dividendenzahlung, bestimmt aus der durch die IvyDB bereitgestellten Dividendenrenditen, ergänzt wird.

Die Auswahl der Vetreter für die Anlageklassen wurde auf Basis der Verfügbarkeit der gehandelten Optionen getroffen. Der S\&P 500 ist als Repräsentant für einen Aktienindex in der Literatur üblich, der TNX Index als langfristige Anleihe hingegen nicht. Jedoch zeigt ein Vergleich der Investition in den TNX Index mit einer Investition in einen entsprechenden Forward ${ }^{8}$ über den out-of-sample Zeitraum eine Korrelation von 0,998. Für den XAU Index als Vetreteter für die Rohstoffklasse wird gegenüber einer direkten Investition in Gold oder Silber eine Korrelationen von 0,884 (Gold) und 0,925 (Silber) bestimmt. ${ }^{9}$ Die Korrelationen zeigen, dass die Vetreteter für die entsprechende Anlageklasse als repräsentativ einzustufen sind.

Die Abbildung 3.1 zeigt für die riskanten Anlageklassen die kumulierten Excess Returns der entsprechenden Vertreter. Es ist zu erkennen, dass im untersuchten Zeitraum sowohl

\footnotetext{
8 Der vergleichbare Forward hat eine Restlaufzeit von weniger als einen Monat (als Approximation für den Spotpreis). Die Lieferung entspricht einer US Treasury Note mit einer Restlaufzeit von 6-10 Jahren. Die Forwardkurse stammen aus Datastream.

9 Die Korrelationen basieren auf Spotpreisen der CME.
} 


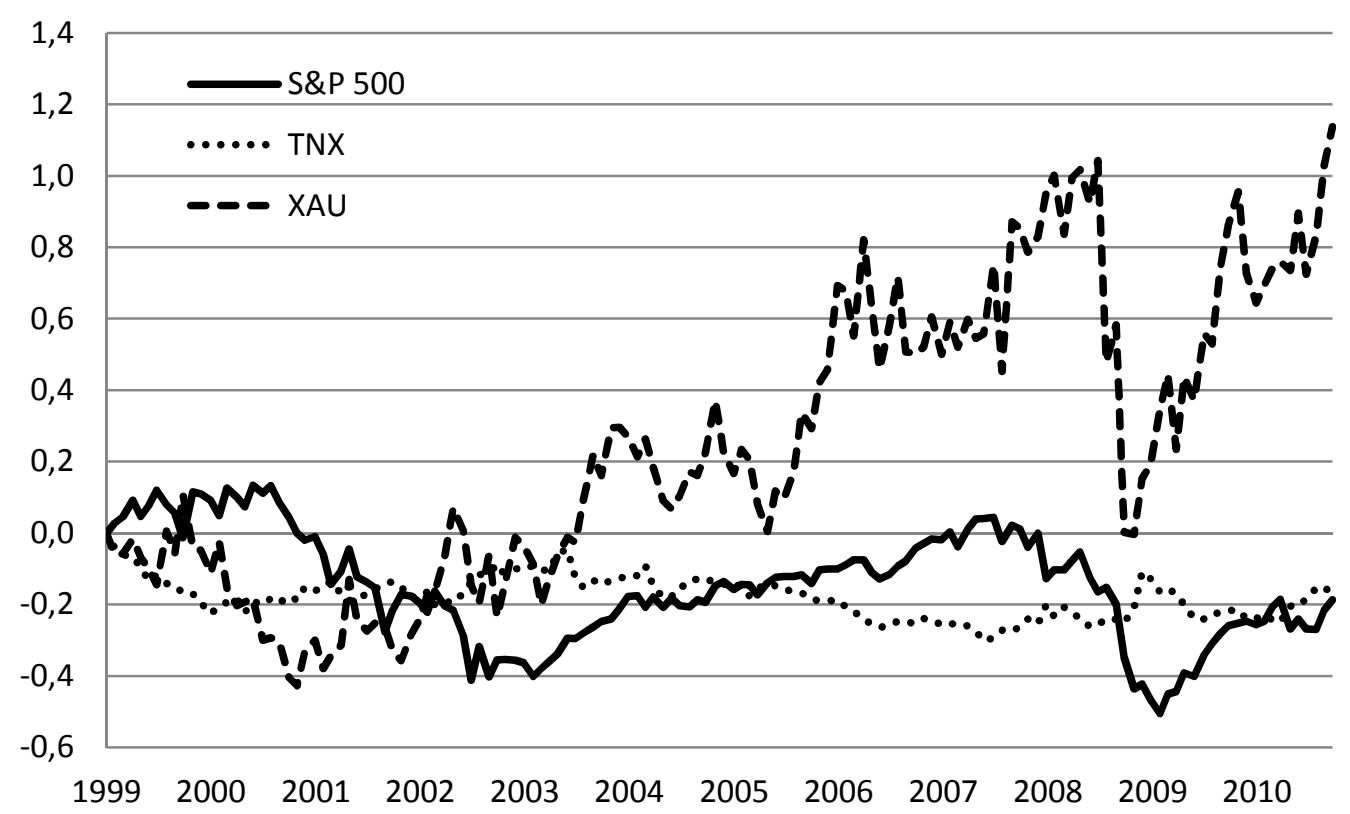

Abbildung 3.1: Diese Abbildung zeigt die kumulierten Excess Returns auf monatlicher Basis für den S\&P 500 Total Return Index, den TNX Index und den XAU Index. Der Zeitraum beträgt Februar 1999 bis Oktober 2010.

der S\&P 500 Total Return Index als auch der TNX Index kumuliert eine geringere Verzinsung als die risikofreie Anlage hatten. Der XAU Index liefert hingegen eine positive Verzinsung, wobei eine starke Schwankung (Volatilität) in der Zeitreihe zu beobachten ist.

\subsection{Implizite Verteilungen aus Optionsmarktdaten}

Dieser Abschnitt beschreibt Techniken zur Extraktion und Risikoadjustierung impliziter Verteilungen aus Optionsmarktdaten. Zum einen werden die wesentlichen theoretischen Schritte kurz dargestellt, zum anderen wird für einen Stichtag die Umsetzung beispielhaft vorgeführt. Der Abschnitt schließt mit der Formulierung einer multidimensionalen Dichte, welche schließlich in der strategischen Asset Allokation mit impliziten Informationen zur Anwendung kommt.

Die Konstruktion einer impliziten multidimensionalen Verteilung und somit die Gewinnung der impliziten Informationen für die optimale Asset Allokation wird in drei aufeinander aufbauenden Schritten geleistet:

(i) Extraktion der risikoneutralen Randdichten der riskanten Anlagewerte aus gehandelten Optionsmarktdaten, 
(ii) Risikoadjustierung bzw. Transformation der risikoneutralen Randdichten in das physische Maß,

(iii) Konstruktion der multidimensionalen Renditeverteilung.

Die Basis für die drei Schritte bilden die Methodiken von Breeden und Litzenberger (1978) zur modellfreien Bestimmung der risikoneutralen Dichte aus einem Kontinuum von Optionen und von Bliss und Panigirtzoglou (2004) zur Risikoadjustierung bzw. Überführung der risikoneutralen Dichte in das physische Maß.

\section{Extraktion der risikoneutralen Randdichten der riskanten Anlagewerte aus gehandelten Optionsmarktdaten}

Breeden und Litzenberger (1978) zeigen unter der Annahme vollständiger und insbesondere arbitragefreier Märkte wie aus einer Butterflyspread-Strategie die risikoneutrale Dichte für ein Asset aus Optionsmarktdaten gewonnen werden kann. Die wesentliche Idee soll kurz erläutert werden.

Gesucht ist das risikoneutrale Wahrscheinlichkeitsmaß $Q$ mit Dichte $q$ zum Zeitpunkt $t$ für den Kurs $S_{t+\tau}$ im Zeitpunkt $t+\tau$. Mit $C(t, \tau, x)$ ist der Wert einer europäischen CallOption zum Zeitpunkt $t$ mit der Laufzeit $\tau$ und dem Ausübungspreis $x$ bezeichnet. Ein Butterflyspread gemäß Breeden und Litzenberger (1978) entspricht einem Portfolio aus den folgenden vier europäischen Call-Positionen

$$
\text { long } C(t, \tau, x-\Delta x)+2 \text { short } C(t, \tau, x)+\operatorname{long} C(t, \tau, x+\Delta x)
$$

Dieses Portfolio liefert im Grenzwert für $\Delta x \rightarrow 0$ zum Zeitpunkt $t+\tau$ eine Auszahlung von $\Delta x$ für $S_{t+\tau}=x$ und 0 sonst. Durch Normierung (Anpassung der Positionen im Portfolio) mit $\frac{1}{\Delta x}$ entspricht das Auszahlungsprofil des Portfolios im Grenzwert der Indikatorfunktion

$$
\mathbb{1}_{\{x\}}\left(S_{t+\tau}\right) .
$$

Der Wert dieses Auszahlungsprofils zum Zeitpunkt $t$ wird gemäß risikoneutraler Bewertung durch die diskontierte risikoneutrale Wahrscheinlichkeit gegeben, dass der Kurs des Assets in $t+\tau$ mit $x$ übereinstimmt

$$
E^{Q}\left\{e^{-r \tau} \mathbb{1}_{\{x\}}\left(S_{t+\tau}\right)\right\}=e^{-r \tau} Q\left\{S_{t+\tau}=x\right\}
$$

wobei $r$ den stetigen risikolosen Zinssatz bezeichnet. Aus Duplikationsgründen entspricht dies gerade dem Preis des normierten Butterflyspreads

$$
\frac{C(t, \tau, x-\Delta x)-2 C(t, \tau, x)+C(t, \tau, x+\Delta x)}{\Delta x} .
$$


Zusammenfassend kann nun die risikoneutrale Dichte $q$ an der Stelle $x$ gewonnen werden

$$
\begin{aligned}
q(x) & =\lim _{\Delta x \rightarrow 0} \frac{Q\{x-\Delta x \leq x \leq x+\Delta x\}}{\Delta x} \\
& =\lim _{\Delta x \rightarrow 0} e^{r \tau} \frac{C(t, \tau, x-\Delta x)-2 C(t, \tau, x)+C(t, \tau, x+\Delta x)}{(\Delta x)^{2}} .
\end{aligned}
$$

Dies entspricht gerade dem Differentialquotienten zweiter Ordnung, womit für die risikoneutrale Dichte

$$
q(x)=e^{r \tau} \frac{d^{2}}{d K^{2}} C(t, \tau, K)_{\mid K=x}
$$

gilt. Die Darstellung besagt, dass die aufgezinste zweite Ableitung nach dem Ausübungspreis einer europäischen Call-Option gerade der risikoneutralen Dichtefunktion entspricht. Die beschriebene Methodik verwendet keine Annahme an die Verteilung oder Struktur des Assets $S_{t+\tau}$ und kann daher als modellfrei bezeichnet werden.

Für die Umsetzung der eingeführten Methodik werden europäische Call-Optionen zu beliebigen Ausübungspreisen bzw. ein Kontinuum von Call-Preisen benötigt. Marktdaten liegen jedoch zum einem nur zu diskreten Punkten, zum anderen für einige Basiswerte nur in der amerikanischen Form vor. Für den Fall europäischer Optionen wäre die direkte Verbindung der Marktpreise mittels numerischer Methoden eine intuitive Umsetzung. Jedoch liefert dieses Verfahren eine instabile Schätzung der risikoneutralen Dichte. In der Literatur hat sich daher der Umweg über die implizite Volatilität etabliert.

Vor diesem Hintergrund werden kurz die wesentlichen Schritte zur Bestimmung der risikoneutralen Dichte mittels einer geglätteten Volatilitätsoberfläche beschrieben. ${ }^{10}$

(i) Bestimmung der Volatilitätsoberfläche

Aus den beobachtbaren Marktpreisen wird mittels der Black-Scholes Formel oder im Falle amerikanischer Optionen mit Hilfe eines Binomialmodells die implizite Volatilität extrahiert (vgl. Shimko (1993)). Die diskreten Punkte in der Volatilitäts - Ausübungspreis - Laufzeit Oberfläche werden unter Zuhilfenahme numerischer Verfahren verbunden und an den Rändern entweder numerisch fortgesetzt oder als konstant angenommen. Durch Anwendung der Black-Scholes Formel können diese Werte wiederum für eine vorgegebene Laufzeit in die Call-Preis - Ausübungspreis Ebene für europäische Optionen überführt werden.

Eine Weiterführung der aufgezeigten Methodik ist der sogenannte Delta-Ansatz (vgl. Malz (1997a), Malz (1997b)). Hierbei wird die Volatilitätsoberfläche in die Volatilitäts - Delta - Laufzeit Oberfläche überführt. Dies hat insbesondere den Vorteil, dass

10 Für einen ausführlichen Literaturüberblick vgl. Bliss und Panigirtzoglou (2002). 


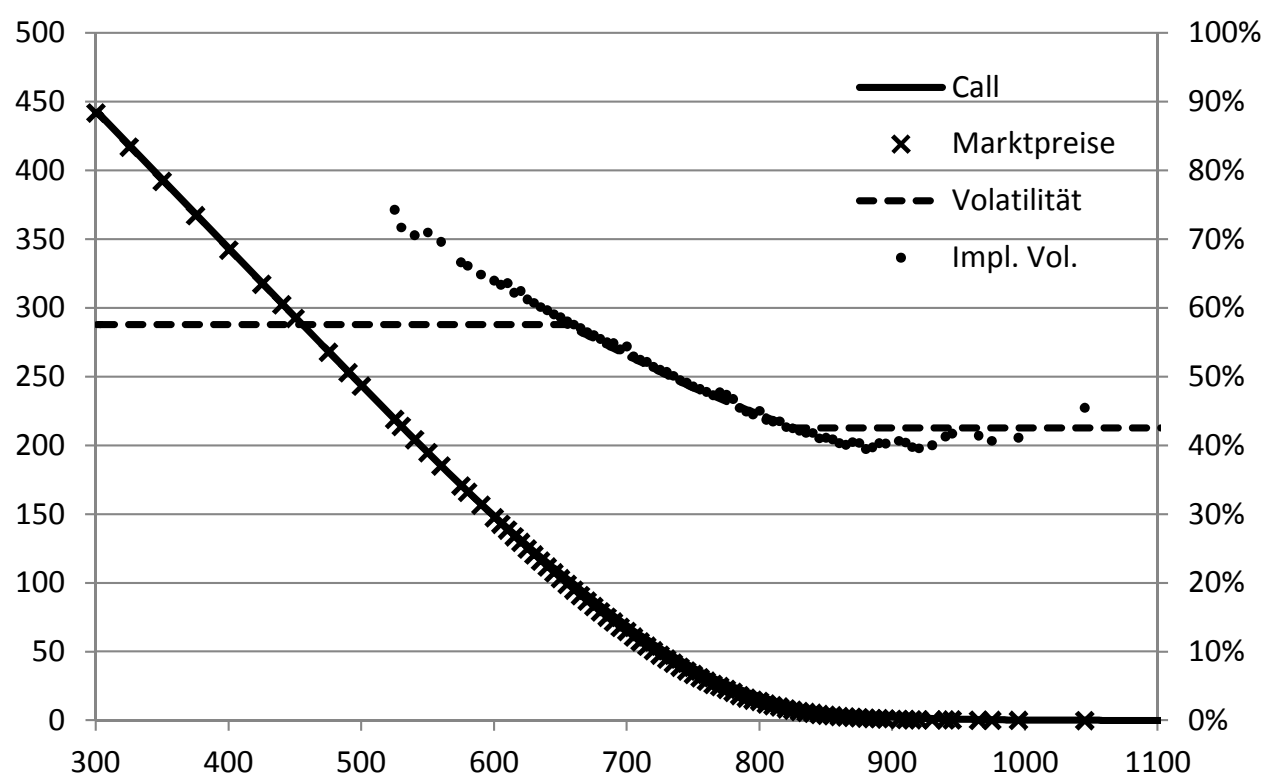

Abbildung 3.2: Diese Abbildung zeigt für den S\&P 500 die Marktpreise (linke Skala) sowie für impliziten Volatilitäten (rechte Skala) das konstruierte Kontinuum bzgl. des Ausübungspreises. Die Volatilitäten sind annualisiert. Die Marktdaten stammen vom 23.02.2009. Die Laufzeit der Optionen ist ein Monat. Der Kurs vom 23.02.2009 des S\&P 500 beträgt 743,33 .

die Stützstellen bei steigender Restlaufzeit gleichmäßiger auf der Oberfläche verteilt sind. Die resultierenden diskreten Punkte können wiederum numerisch verbunden ${ }^{11}$ und mit der Black-Scholes Formel in kontinuierliche Call-Preise überführt werden.

Für diese Studie sind für konstante diskrete Delta-Werte die Volatilitätsoberflächen (bereinigt um Dividenden) in der IvyDB verfügbar. Mit einer Cubic-Spline Glättung werden diese Punkte zu einem Kontinuum verbunden und mit der Black-Scholes Formel in die entsprechenden Call-Preise übersetzt.

Die Abbildung (3.2) zeigt grafisch die umgesetzte Methodik. Die implizite Volatilität wurde für Ausübungspreise weit aus dem bzw. weit im Geld konstant fortgesetzt. Zwischen den Marktpreisen und den kontinuierlichen Call-Preisen resultiert aus der Berücksichtigung der Dividendenrendite in der Volatilitätsoberfläche eine geringe Divergenz für Ausübungspreise um den aktuellen Kurs $S_{t}$.

(ii) Gewinnung der risikoneutralen Dichte

Aus der obigen Herleitung muss gemäß Breeden und Litzenberger (1978) die zweite Ableitung der Call-Preise nach dem Ausübungspreis bestimmt werden. Für die Umsetzung wird mit Hilfe von 1000 Stützstellen auf dem Intervall $\left[0,3 \cdot S_{t}\right]$ der Dif-

11 Z. B. mit einer Cubic-Spline Glättung (vgl. Campa, Chang und Reider (1998)). 


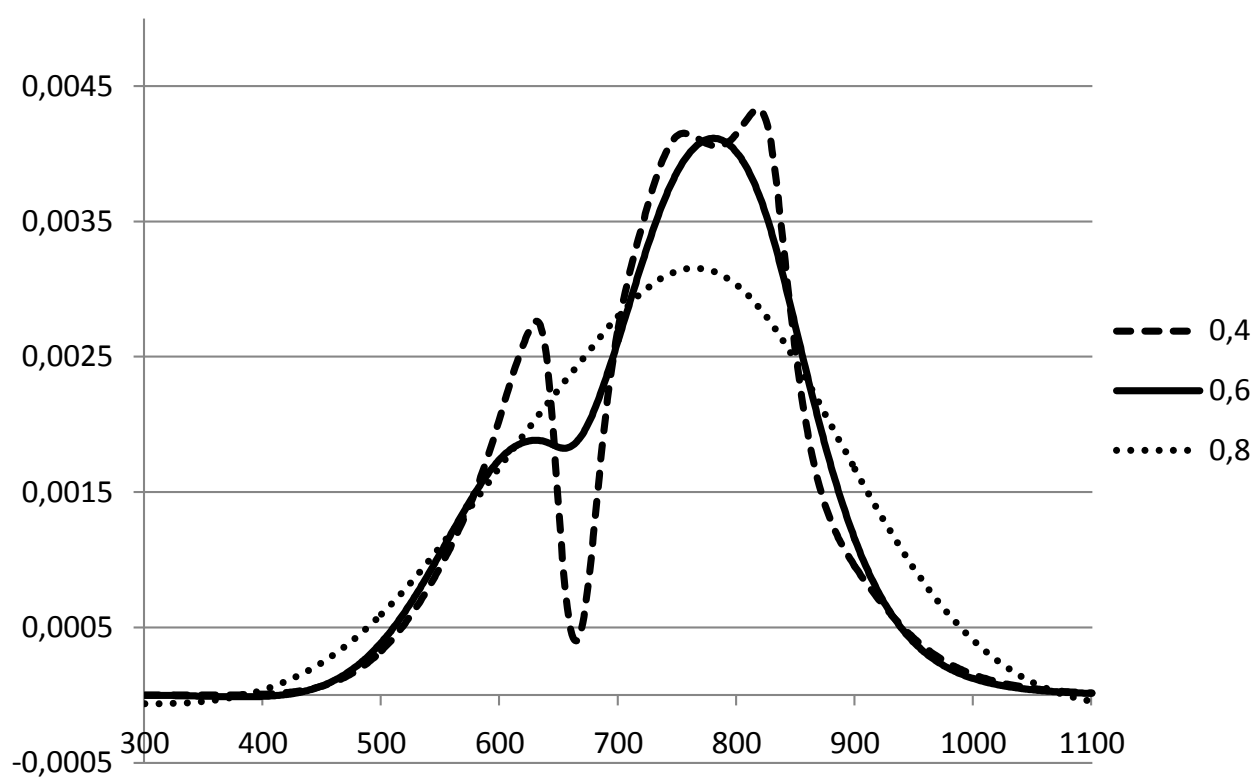

Abbildung 3.3: Diese Abbildung zeigt für den S\&P 500 die mit einem Cubic-Spline Verfahren geglättete zweite Ableitung der Call-Preise nach dem Ausübungspreis. Die Parameter 0,4;0,6;0,8 geben die Stärke der Glättung an. Die Kurven beziehen sich auf Daten von Optionen auf den S\&P 500 vom 23.02.2009 mit einer Laufzeit von einem Monat. Der Kurs vom 23.02.2009 des S\&P 500 beträgt 743,33.

ferentialquotient zweiter Ordnung gebildet und mit numerischen Verfahren zu einer stetigen Funktion zusammengefügt.

Diese Methodik garantiert keine nichtnegativen Werte für die zweite Ableitung und somit auch für die risikoneutrale Dichte. Bliss und Panigirtzoglou (2002) schlagen hierzu vor, eine Cubic-Spline Glättung der Call-Preis - Ausübungspreis Ebene vorzunehmen.

Tabelle 3.1: Einfluss des Glättungsparameters auf die Momente der Verteilung

\begin{tabular}{ccccc}
\hline & ERW & STD & SKEW & KURT \\
\hline 0,40 & 743,66 & 106,13 & $-0,22$ & 2,88 \\
0,60 & 743,66 & 106,13 & $-0,22$ & 2,72 \\
0,80 & 743,63 & 106,16 & $-0,22$ & $-1,79$ \\
\hline
\end{tabular}

Diese Tabelle zeigt den Einfluss des Glättungsparameters auf die Momente der Verteilung. Die Werte beziehen sich auf Daten von Optionen auf den S\&P 500 vom 23.02.2009 mit einer Laufzeit von einem Monat. Der Kurs vom 23.02.2009 des S\&P 500 beträgt 743,33. Schiefe und Kurtosis sind standardisiert.

Die Abbildung (3.3) zeigt wie sich unterschiedliche Glättungsparameter auf die Struktur der risikoneutralen Dichte auswirken. Der Glättungsparameter hat generell nur einen geringen Einfluss auf die Momente der Verteilung, bei starker Glättung $(0,8)$ liefert die Verteilung allerdings eine negative Kurtosis. In Tabelle (3.1) sind die ersten vier Momente für unterschiedliche Glättungsparameter aufgeführt. Zum Vergleich entspricht der risikoneutrale Erwartungswert dem mit dem risikofreien Zinssatz aufgezinstem aktuellem 
Kurs. Für das gewählte Beispiel stellt sich das Ergebnis als $(1+0,04 \%) \cdot 743,33=743,66$ dar. Für den Glättungsparameter 0,8 weicht der Erwartunsgwert empirisch ab, da an den Rändern negative Dichten ermittelt werden.

In der folgenden Studie wurde als Glättungsparameter 0,6 verwendet.

\section{Risikoadjustierung bzw. Transformation der risikoneutralen Randdichten in das physische Maß}

Die gewonnenen risikoneutralen Dichten müssen um Risikoprämien bereinigt werden bzw. in das physische "Real-World"-Maß überführt werden. Dies wird mit Hilfe der Existenz eines repräsentativen Investors, welche zum Beispiel in Constantinides (1982) oder in Kapitel 5 von Huang und Litzenberger (1988) diskutiert wird, geleistet.

Der repräsentative Investor stellt die "fairen Preise" für die riskanten Anlagewerte am Markt. In einem Kapitalmarkt bestehend ausschließlich aus einer risikofreien und einer riskanten Anlage ist dieser dadurch charakterisiert, dass er im Gleichgewichtszustand in $t=0$ sein ganzes verfügbares Einkommen in die riskante Anlage investiert und in $t=T$ den Preis der riskanten Anlage konsumiert. Bei Zugrundelegung eines vollständigen Marktes und angemessenen Annahmen hinsichtlich Präferenzen und Einkommensschwankungen (vgl. Aït-Sahalia und Lo (2000)) kann ein repräsentativer Investor mit Nutzenfunktion $U$ eingeführt werden.

Mittels des repräsentativen Investors kann die adäquate Risikoadjustierung bestimmt und der Übergang von der risikoneutralen Dichte in das physische Maß gewährleistet werden. Dieser Zusammenhang soll kurz in einer Heuristik dargestellt werden. ${ }^{12}$

Aus der arbeitragefreien Preistheorie ist bekannt, dass der Preis $p_{f}$ für einen beliebigen Payoff $f\left(S_{T}\right)$ zum Zeitpunkt $T$ bestimmt werden kann durch

$$
p_{f}=e^{-r T} E^{Q}\left\{f\left(S_{T}\right)\right\},
$$

wobei $r$ den stetigen risikolosen Zinssatz bezeichnet. Dieser Preis muss mit der Bepreisung mittels des stochastischen Diskontfaktors des repräsentativen Investors übereinstimmen (vgl. Cochrane (2005))

$$
p_{f}=E^{P}\left\{\beta \frac{U^{\prime}\left(C_{T}\right)}{U^{\prime}\left(C_{0}\right)} f\left(S_{T}\right)\right\}
$$

$\overline{12}$ Für eine detaillierte Herleitung siehe z. B. Aït-Sahalia und Lo (2000) oder Huang und Litzenberger (1988). 
wobei $\beta$ der subjektive Diskontfaktor und $C_{0}$ der Konsum in $t=0$ ist. Entscheidend für die Konstruktion des Überganges vom risikoneutralen zum physischen Wahrscheinlichkeitsmaß ist, dass der Preis bzgl. des arbitragefreien Ansatzes unter dem risikoneutralen Maß $Q$ bestimmt wird und derjenige über den repräsentativen Investor unter dem physischen Maß $P$. Aufgrund der charakterisierenden Eigenschaft des repräsentativen Investors gilt weiter $C_{T}=S_{T}$.

Da $f$ eine beliebige Funktion ist und somit insbesondere Indikatorfunktionen beinhaltet, folgt

$$
q \cdot e^{-r T}=\beta \cdot \frac{U^{\prime}\left(S_{T}\right)}{U^{\prime}\left(C_{0}\right)} \cdot p
$$

wobei $q$ und $p$ die Dichtefunktionen bzgl. des risikoneutralen und physischen Maßes bezeichnen.

Um sicherzustellen, dass $p$ eine Wahrscheinlichkeitsdichte ist, wird die Funktion normiert. Dabei kürzen sich die konstanten Terme $\beta, e^{-r T}$ und $U^{\prime}\left(C_{0}\right)$ aus dem Kalkül heraus. Abschließend ergibt sich folgender Zusammenhang zwischen risikoneutraler und physischer Dichte

$$
p(x)=\frac{\frac{q(x)}{U^{\prime}(x)}}{\int \frac{q(x)}{U^{\prime}(x)} d x} .
$$

Beispielhaft wird folgend die Transformation im Rahmen eines repräsentativen Investors mit CRRA Nutzenfunktion und log-normalverteilten Kursen illustriert. Das Beispiel zeigt die Wirkung der Transformation auf die zugrundeliegenden Verteilungen auf.

Der Kurs des Assets $S_{T}$ unter dem risikoneutralen Maß ist $\mathcal{L N}\left(\mu, \sigma^{2}\right)$ verteilt und der repräsentative Investor wird charakterisiert durch eine CRRA Nutzenfunktion mit Risikoaversionsparameter $\gamma \in \mathbb{R}(\operatorname{vgl}$. (2.6)). Dann gilt

$$
\begin{aligned}
q(x) & =\frac{1}{\sqrt{2 \pi} \sigma x} \exp \left(-\frac{(\log x-\mu)^{2}}{2 \sigma^{2}}\right) \quad \text { für } x>0, \\
U^{\prime}\left(S_{T}\right) & =S_{T}^{-\gamma} .
\end{aligned}
$$

Insgesamt ist die physische Dichte gemäß (3.1) dann proportional zu

$$
\begin{aligned}
p(x) & \propto \frac{x^{\gamma}}{\sqrt{2 \pi} \sigma x} \exp \left(-\frac{(\log x-\mu)^{2}}{2 \sigma^{2}}\right) \\
& =\frac{1}{\sqrt{2 \pi} \sigma x} \exp \left(-\frac{(\log x-\mu)^{2}-2 \sigma^{2} \gamma \log x}{2 \sigma^{2}}\right) \\
& =\exp \left(2 \mu \gamma \sigma^{2}-\gamma^{2} \sigma^{4}\right) \frac{1}{\sqrt{2 \pi} \sigma x} \exp \left(-\frac{\left(\log x-\left(\mu+\gamma \sigma^{2}\right)\right)^{2}}{2 \sigma^{2}}\right)
\end{aligned}
$$


Da $\int \frac{q(x)}{U^{\prime}(x)} d x=\exp \left(2 \mu \gamma \sigma^{2}-\gamma^{2} \sigma^{4}\right)$ ist, folgt, dass $S_{T}$ auch unter dem physischen Maß $\log$-normalverteilt ist, und zwar mit Parametern $\left(\mu+\gamma \sigma^{2}, \sigma^{2}\right)$.

Somit sind die Log-Renditen unter beiden Maßen normalverteilt. Die Volatilität bleibt von der Risikoadjustierung unberührt und ist unter beiden Maßen gleich. Die Risikoadjustierung steuert somit ausschließlich den Erwartungswert der Log-Renditen und hat keinen Einfluss auf die anderen Momente der Verteilung, insbesondere nicht auf die Varianz, Schiefe und Kurtosis.

Tabelle 3.2: Einfluss der Risikoadjustierung auf die Momente des Kurses

\begin{tabular}{ccccccc}
\hline RRA & $E^{P}\left\{S_{T}\right\}$ & $\operatorname{Var}^{P}\left\{S_{T}\right\}$ & $E^{P}\left\{R_{T}\right\}$ & $\operatorname{Var}^{P}\left\{R_{T}\right\}$ & $\operatorname{Skew}^{P}\left\{R_{T}\right\}$ & $\operatorname{Kurt}^{P}\left\{R_{T}\right\}$ \\
\hline-4 & 99,53 & 60,94 & $-0,78$ & 0,61 & 0,00 & 3,00 \\
-3 & 100,14 & 61,69 & $-0,17$ & 0,61 & 0,00 & 3,00 \\
-2 & 100,76 & 62,45 & 0,45 & 0,61 & 0,00 & 3,00 \\
-1 & 101,38 & 63,22 & 1,06 & 0,61 & 0,00 & 3,00 \\
0 & 102,00 & 64,00 & 1,67 & 0,61 & 0,00 & 3,00 \\
1 & 102,63 & 64,79 & 2,29 & 0,61 & 0,00 & 3,00 \\
2 & 103,26 & 65,59 & 2,90 & 0,61 & 0,00 & 3,00 \\
3 & 103,89 & 66,40 & 3,51 & 0,61 & 0,00 & 3,00 \\
4 & 104,53 & 67,22 & 4,13 & 0,61 & 0,00 & 3,00 \\
\hline
\end{tabular}

Diese Tabelle zeigt bei gegebener Risikoaversion des repräsentativen Investors den Einfluss der Risikoadjustierung auf die Momente des Kurses $S_{T}$ und der $\log$-Renditen $R_{T}=\log \left(\frac{S_{T}}{S_{0}}\right)$. Die risikoneutrale Dichte wird gegeben durch eine Log-Normalverteilung mit Erwartungswert $E^{Q}\left\{S_{T}\right\}=102$ und Varianz $\operatorname{Var}^{Q}\left\{S_{T}\right\}=64$. Der aktuelle Kurs $S_{0}$ sei 100 .

Tabelle 3.2 zeigt im Rahmen eines konkreten Szenarios den Einfluss der Risikoadjustierung des repräsentativen Investors bei gegebener Risikoaversion auf die Verteilung des Kurses $S_{T}$. Der Kurs $S_{T}$ ist log-normalverteilt mit Erwartungswert $E^{Q}\left\{S_{T}\right\}=102$ und Varianz $\operatorname{Var}^{Q}\left\{S_{T}\right\}=64$. Der aktuelle Kurs $S_{0}$ ist 100 und mit $R_{T}=\log \left(\frac{S_{T}}{S_{0}}\right)$ wird die LogRendite bezeichnet. Der repräsentative Investor wird durch eine CRRA Nutzenfunktion beschrieben.

Falls der repräsentative Investor durch einen Risikoaversionsparameter von $\gamma=0$ charakterisiert wird, demnach risikoneutral ist, stimmt die physische Dichte exakt mit der risikoneutralen überein und somit stehen auch die entsprechenden Momente der Verteilungen in Analogie zueinander. ${ }^{13}$ Durch einen Anstieg des Risikoaversionsparameters erhöht sich die erwartete Log-Rendite des Kurses, die Volatilität bleibt jedoch unberührt. Als Interpretation ist anzuführen, dass ein risikoaverser Investor für dasselbe einzugehende Risiko eine höhere Kompensation, ausgedrückt in der erwarteten Rendite, verlangt. Für

$13 \quad U^{\prime}\left(S_{T}\right)=\left(S_{T}\right)^{0}=1$. 
einen negativen Risikoaversionsparameter ist der repräsentative Investor hingegen risikosuchend und hat daher für dasselbe Risiko eine geringere Renditeforderung.

Zur Transformation der risikoneutralen Dichten in der empirischen Studie wird die Annahme getroffen, dass für jede riskante Anlageklasse, also für den Aktienmarkt, den Anleihemarkt und den Rohstoffmarkt, jeweils ein repräsentativer Investor existiert und diese unabhängig voneinander agieren. Des Weiteren werden die Investoren jeweils durch eine CRRA Nutzenfunktion mit Risikoaversionsparameter $\gamma \in \mathbb{R}$ charakterisiert. Für die vollständige Beschreibung der Nutzenfunktionen der repräsentativen Investoren und somit für die Risikoadjustierung der impliziten Dichten verbleibt die Schätzung der drei unbekannten Risikoaversionsparameter. Hierzu wird dem Ansatz von Bliss und Panigirtzoglou (2004) gefolgt.

Bliss und Panigirtzoglou (2004) benutzen hierfür die Berkowitz Statistik ${ }^{14}$, die insbesondere zur Bestimmung der Anpassungsgüte von vollständigen Verteilungen bei geringer Anzahl von Beobachtungen geeignet ist. ${ }^{15} \mathrm{Im}$ Wesentlichen sind für die Durchführung zwei aufeinander folgende Schritte zu betrachten. ${ }^{16}$

Sei $\gamma \in \mathbb{R}$ zum Zeitpunkt $t=T$ fixiert. Mit $f_{0}, \ldots, f_{T-1}$ werden die mit einer CRRA Nutzenfunktion und Risikoaversionsparameter $\gamma$ transformierten risikoneutralen Dichten sowie mit $X_{1}, \ldots, X_{T}$ die entsprechenden historischen Kurse des riskanten Assets bezeichnet. Die Kurse entsprechen den Realisationen des physischen bzw. des risikoadjustierten impliziten Maßes und werden als paarweise stochastisch unabhängig angenommen. Es ist im Folgenden zu prüfen, ob $\gamma$ die richtige Transformation liefert und somit den repräsentativen Investor beschreibt.

(i) Konstruktion der zu untersuchenden Zeitreihe

Unter der Null-Hypothese, dass $\gamma$ die richtige Transformation liefert, stimmt die mit $\gamma$ risikoadjustierte implizite Dichte $f_{t}$ zum Zeitpunkt $t$ mit der wahren (unbekannten, physischen) Dichte überein. Eine Transformation der Realisationen über die inverse Verteilungsfunktion

$$
y_{t}=\int_{-\infty}^{X_{t}} f_{t-1}(u) d u \quad \text { für } t=1, \ldots, T
$$

liefert unter der Null-Hypothese eine auf $[0,1]$ rechteckverteilte Zeitreihe.

14 Vgl. Berkowitz (2001).

15 Vgl. die Diskussion in Bliss und Panigirtzoglou (2004).

16 Zur Vergleichbarkeit ist die Notation aus Bliss und Panigirtzoglou (2004) in den Grundzügen übernommen worden. 
Anstatt die gewonnene Zeitreihe der $y_{t}$ direkt auf ihre Güte zu untersuchen, schlägt Berkowitz eine weitere parametrische Transformation mit Hilfe der Verteilungsfunktion der Standardnormalverteilung vor, und zwar

$$
z_{t}=\Phi^{-1}\left(y_{t}\right)=\Phi^{-1}\left(\int_{-\infty}^{X_{t}} f_{t-1}(u) d u\right) \quad \text { für } t=1, \ldots, T \text {. }
$$

Unter der Null-Hypothese sind die $z_{t}$ nun standardnormalverteilt.

(ii) Beurteilung der Güte der konstruierten Zeitreihe

Aus der im ersten Schritt konstruierten Zeitreihe der $z_{t}$ wird gemäß Berkowitz ein AR(1)-Prozess geschätzt:

$$
z_{t}-\mu=\rho\left(z_{t-1}-\mu\right)+\epsilon_{t}
$$

Die Parameter werden mittels eines Maximum Likelihood Ansatzes ermittelt und jeweils mit einem Likelihood Ratio Test auf ihre Werte geprüft. Im Einzelnen werden die Parameter unter der Null-Hypothese durch $\mu=0, \rho=0$ und $\operatorname{Var}\left(\epsilon_{t}\right)=1$ gegeben. Bezeichnet $L\left(\mu, \sigma^{2}, \rho\right)$ die Log-Likelihood Funktion, ${ }^{17}$ wird der entsprechende Likelihood Ratio Test gegeben durch

$$
L R_{3}=2\left[L(0,1,0)-L\left(\hat{\mu}, \hat{\sigma^{2}}, \hat{\rho}\right)\right]
$$

Der Likelihood Ratio Test ist unter der Null-Hypothese Chi-Quadrat mit drei Freiheitsgraden verteilt.

Mit Hilfe dieser Systematik können die Risikoaversionsparameter der repräsentativen Investoren gewählt werden. Für unterschiedliche Risikoaversionsparameter $\gamma \in \mathbb{R}$ und einem zuvor festgelegten Schätzhorizont werden obige Schritte durchgeführt und der Likelihood Ratio Test ausgewertet. Der Risikoaversionsparameter, welcher die beste Anpassung im Sinne des Likelihood Ratio Tests liefert, wird mit der Risikoaversion des entsprechenden repräsentativen Investors gleichgesetzt.

In der vorliegenden Studie wird für die Schätzung jeweils das Gitter zwischen [-5,5] in 0,01 Schritten evaluiert und wie oben beschrieben ausgewertet. Der Schätzhorizont wiederum ist auf die letzten 36 Monate festgelegt. Tabelle 3.3 zeigt beispielhaft die erhaltenen Werte aus dem Likelihood Ratio Test für den Aktienindex.

17 Für eine explizite Darstellung der Log-Likelihood Funktion siehe Berkowitz (2001). 
Tabelle 3.3: Auswertungen des Likelihood Ratio Tests für ausgewählte Risikoaversionsparameter

\begin{tabular}{cccccc}
\hline & 0,0 & 0,2 & 0,4 & 0,6 & 0,8 \\
\hline-3 & $0,1294 \%$ & $0,1429 \%$ & $0,1622 \%$ & $0,1893 \%$ & $0,2268 \%$ \\
-2 & $0,1201 \%$ & $0,1153 \%$ & $0,1138 \%$ & $0,1154 \%$ & $0,1205 \%$ \\
-1 & $0,2006 \%$ & $0,1689 \%$ & $0,1587 \%$ & $0,1411 \%$ & $0,1285 \%$ \\
-0 & $0,6192 \%$ & $0,4797 \%$ & $0,3766 \%$ & $0,3000 \%$ & $0,2431 \%$ \\
0 & $0,6192 \%$ & $0,8084 \%$ & $1,0655 \%$ & $1,4153 \%$ & $1,8914 \%$ \\
1 & $2,5381 \%$ & $3,4149 \%$ & $4,5990 \%$ & $6,1894 \%$ & $8,3118 \%$ \\
2 & $11,1200 \%$ & $14,7978 \%$ & $19,5553 \%$ & $25,6201 \%$ & $33,2182 \%$ \\
3 & $42,5226 \%$ & $53,6124 \%$ & $66,3253 \%$ & $80,0464 \%$ & $93,1732 \%$ \\
\hline
\end{tabular}

Diese Tabelle zeigt Auswertungen des Likelihood Ratio Tests für ausgewählte Risikoaversionsparameter mit Werten zwischen [-3,8;3,8]. Die Anzahl der Beobachtungen für die Schätzung des AR(1)-Prozesses beträgt 36. Die Werte beziehen sich auf den Stichtag 23.02.2009 für die Schätzung des Risikoaversionsparameters des S\&P 500.

Das untersuchte Gitter liefert eine nach oben geöffnete Parabel (vgl. Tabelle 3.3). Als Risikoaversionsparameter des repräsentativen Investors wird das Minimum des Gitters gewählt und beträgt in diesem Fall -2,4. Der repräsentative Investor für den S\&P 500 wird in diesem Fall als risikosuchend eingestuft. Die Nutzenfunktion ist über den Aversionsparameter vollständig charakterisiert und kann zur Bestimmung der vollständigen physischen Dichte gemäß (3.1) genutzt werden.

Tabelle 3.4: Einfluss der Risikoadjustierung auf die Momente der risikoneutralen Verteilung

\begin{tabular}{lcccc}
\hline & ERW & VAR & SKEW & KURT \\
\hline RRA $=0$ & 743,66 & 106,13 & $-0,22$ & 2,72 \\
RRA $=-2,4$ & 704,02 & 110,11 & 0,04 & 2,12 \\
\hline
\end{tabular}

Diese Tabelle zeigt den Einfluss der Risikoadjustierung auf die Momente der risikoneutralen Verteilung. Die Werte beziehen sich auf Daten von Optionen auf den S\&P 500 vom 23.02.2009 mit einer Laufzeit von einem Monat. Der Kurs vom 23.02.2009 des S\&P 500 beträgt 743,33. Schiefe und Kurtosis sind standardisiert. Der Risikoaversionsparameter des repräsentativen Investors, geschätzt aus der Berkowitz Statistik, beträgt $-2,4$.

Abbildung 3.4 und Tabelle 3.4 zeigen den Einfluss der Risikoadjustierung auf die Struktur der Verteilung. Die Adjustierung hat hierbei einen wesentlichen Einfluss auf den Erwartungswert und steuert somit insbesondere die erwartete Rendite. Der repräsentative Investor antizipiert für dieses Beispiel fallende Kurse. Dies steht auch im Einklang mit der Schätzung des negativen Risikoaversionsparameters von -2,4.

Um abschließend eine vollständige Zeitreihe des Risikoaversionsparameters zu erhalten, wurde der vormals festgelegte Horizont von 36 Monaten konstant gehalten und mit einem Rolling Window weitergeführt. Dies bedeutet insbesondere, dass eine zeitvariable Risi- 


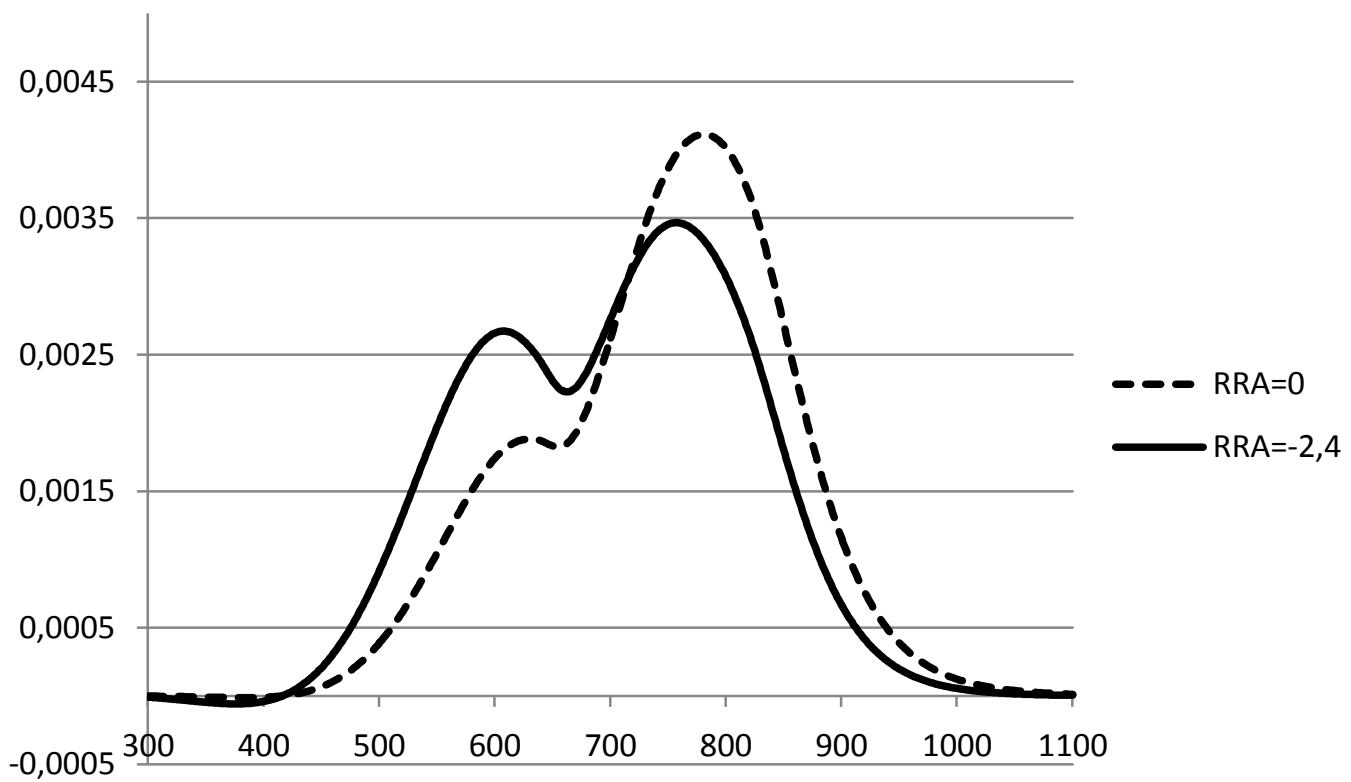

Abbildung 3.4: Diese Abbildung zeigt den Einfluss der Risikoadjustierung auf die Struktur der risikoneutralen Verteilung. Die Kurven beziehen sich auf Daten von Optionen auf den S\&P 500 vom 23.02.2009 mit einer Laufzeit von einem Monat. Der Kurs vom 23.02.2009 des S\&P 500 beträgt 743,33. Der Risikoaversionsparameter des repräsentativen Investors, geschätzt aus der Berkowitz Statistik, beträgt -2,4.

koaversion der repräsentativen Investoren unterstellt wird. Abbildung 3.5 zeigt für den untersuchten Zeitraum die abgeleiteten Risikoaversionsparameter auf Basis der CRRA Nutzenfunktion für die Anlagewerte S\&P 500, TNX Index und XAU Index.

Die Abbildung zeigt auf, dass für den S\&P 500 fast ausschließlich negative Werte und damit negative Excess Returns antizipiert werden. Dieses Ergebnis ist im Einklag mit den realisierten Excess Returns aus Abbildung 3.1. Aus der Risikoaversion des TNX Indexes leitet sich die erwartete Änderung des Zinssatzes bzw. der Yield-To-Maturity ab. Eine positive Risikoaversion bedeutet dabei für den Wert des Zero-Kupon Bonds eine erwartete negative Kursentwicklung.

Die risikoneutralen Randdichten der einzelnen riskanten Anlagewerte sind damit aus Optionsmarktdaten modellfrei abgeleitet (Schritt i) und in das physische Maß transformiert worden (Schritt ii). Es verbleibt, die gemeinsame Renditeverteilung der riskanten Anlagewerte zu modellieren und deren Abhängigkeitsstruktur zu wählen.

\section{Konstruktion der multidimensionalen Renditeverteilung}

Die Konstruktion der gemeinsamen Renditeverteilung und somit insbesondere die Modellierung der Abhängigkeiten zwischen den Assets erfolgt in zwei Schritten. In einem 


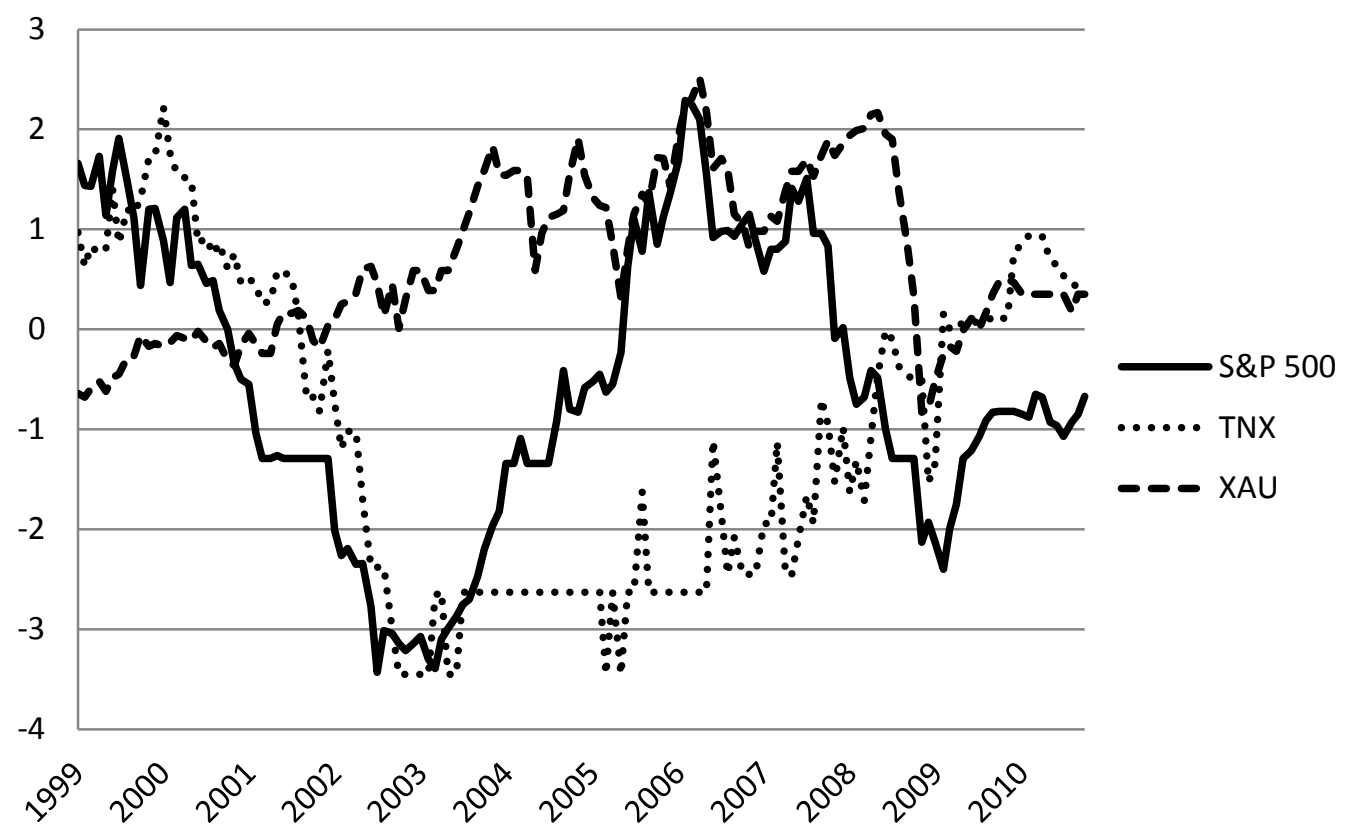

Abbildung 3.5: Diese Abbildung zeigt die geschätzten Risikoaversionsparameter für den S\&P 500 Index, TNX Index und XAU Index. Der Zeitraum der Studie beträgt Februar 1999 bis Oktober 2010.

ersten Schritt werden die Verteilungen der riskanten Asset-Kurse in die entsprechenden Renditeverteilungen mit Hilfe einer Substitution überführt.

$$
\begin{aligned}
P\left\{\frac{S_{t+\tau}-S_{t}}{S_{t}} \leq t^{\prime}\right\} & =P\left\{S_{t+\tau} \leq S_{t}\left(1+t^{\prime}\right)\right\} \\
& =\int_{0}^{S_{t}\left(1+t^{\prime}\right)} p(x) d x \\
& =\int_{-1}^{t^{\prime}} \underbrace{S_{t} \cdot p\left(S_{t}(1+x)\right)}_{\text {Dichte der Rendite }} d x
\end{aligned}
$$

wobei in der letzten Zeile mit der Funktion $z \mapsto S_{t}(1+z)$ substituiert wurde. In einem zweiten Schritt werden die Randdichten der Renditen zu einer gemeinsamen Verteilung verbunden. Hierzu kann auf die Copula Theorie bzw. Sklar's Theorem aufgebaut werden, welche besagt, dass multidimensionale Dichten im Falle von stetigen Verteilungen eindeutig durch ihre Randdichten und einer entsprechenden Copula ausgedrückt werden können. ${ }^{18}$ Dies bedeutet im Rahmen der strategischen Asset Allokation, dass die multi-

18 Vgl. z. B. Patton (2004). 
dimensionale Renditeverteilung $p\left(R_{\mathrm{S} \& \mathrm{P} 500}, R_{\mathrm{TNX}}, R_{\mathrm{XAU}}\right)$ für die riskanten Anlageklassen ausgedrückt werden kann durch

$$
\begin{aligned}
p\left(R_{\mathrm{S} \& \mathrm{P} 500}, R_{\mathrm{TNX}}, R_{\mathrm{XAU}}\right)= & p\left(R_{\mathrm{S} \& \mathrm{P} 500}\right) \cdot p\left(R_{\mathrm{TNX}}\right) \cdot p\left(R_{\mathrm{XAU}}\right) \\
& \cdot c\left(F\left(R_{\mathrm{S} \& \mathrm{P} 500}\right), F\left(R_{\mathrm{TNX}}\right), F\left(R_{\mathrm{XAU}}\right)\right),
\end{aligned}
$$

wobei $p(\cdot)$ die Dichte von $(\cdot), c(\cdot)$ die Dichte der Copula und $F(\cdot)$ die Verteilungsfunktion von $(\cdot)$ angibt. Umgekehrt gelesen, kann bei bekannter Copula mit Hilfe der Randdichten die vollständige multidimensionale Verteilung abgeleitet werden. Die Dichte der Copula $c(\cdot)$ beinhaltet dabei die gesamte Abhängigkeitsstruktur unter den Assets. Diese kann nicht direkt aus Optionsmarktdaten abgeleitet werden, da zum einen Optionen auf mehr als ein Underlying entweder nicht gehandelt werden oder nicht verfügbar sind, zum anderen noch keine Techniken zur Extraktion einer modellfreien Copula erforscht wurden. Auch eine Risikoadjustierung und Überführung in das phyische Maß ist unklar, da auch Risikoprämien in den Abhängigkeitsstrukturen, wie die Korrelations-Risikoprämie, existieren.

Um dieser Problematik zu begegnen wird im Folgenden ein parametrischer Ansatz verfolgt und eine t-Copula für die Abhängigkeitsstruktur verwendet. Als zusätzliche Parameter müssen eine Korrelationsmatrix und die Anzahl der Freiheitsgrade geschätzt werden. Hierzu wird aus einem Rolling Window der Tagesrenditen der letzten drei Monate eine multidimensionale t-Verteilung mit Hilfe eines Maximum Likelihood Ansatzes geschätzt. ${ }^{19}$ Die geschätzten Parameter werden dann für die Copula verwendet. Eine Risikoadjustierung muss nicht vorgenommen werden, da die Copula unter dem physischen Maß und nicht unter dem risikoneutralen konstruiert wird. Abbildung 3.6 zeigt beispielhaft die auf diese Weise konstruierte multidimensionale Renditeverteilung für den S\&P 500 und dem TNX Index.

\subsection{Methodik der empirischen Studie}

Dieser Abschnitt stellt den Aufbau und das Vorgehen der empirischen Studie vor. Zunächst wird das Asset Allokationsproblem für die betrachteten Investoren der Studie beschrieben. Es folgt die Vorstellung der Performance-Kennzahlen und der Benchmark Strategien.

19 R-Paket: QRMLib. 


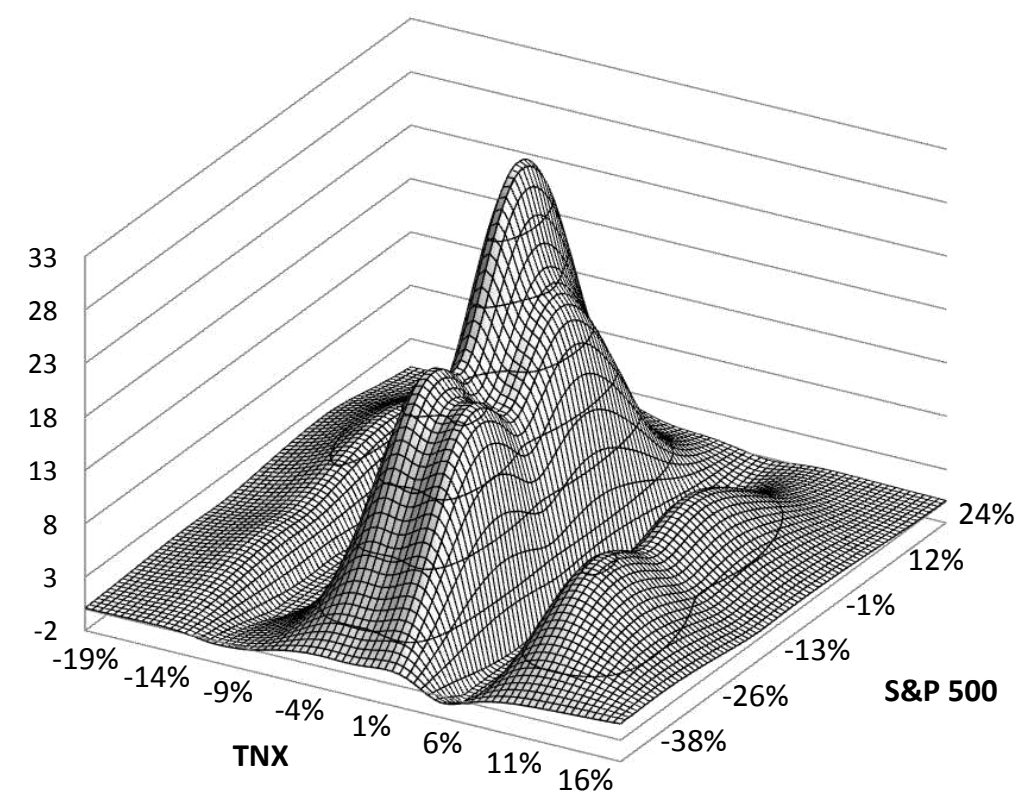

Abbildung 3.6: Diese Abbildung zeigt die 2-dimensionale physische Dichte für den S\&P 500 und den TNX Index am 23.02.2009. Für die t-Copula wurde eine Korrelation von -0,291 mit 8,127 Freiheitsgraden geschätzt.

\subsubsection{Bestimmung der optimalen Asset Allokation}

Für die empirische Studie werden, wie in Kapitel 2 beschrieben, Investoren mit CRRA Nutzenfunktionen mit Risikoaversionsparametern $\gamma=2,4,6$ und 8 untersucht. Der Anlagehorizont der Investoren beträgt einen Monat und die Investoren haben nicht die Möglichkeit, Short-Positionen bei ihrer Asset Allokation einzugehen und unterliegen somit einer strikten Leerverkaufsbeschränkung.

Für die Bestimmung der optimalen Portfoliogewichte werden die Investoren das Optimierungsproblem (2.7) lösen. Hierzu sind der Erwartungswert und die Matrizen $M_{2}, M_{3}, M_{4}$ zum jeweiligen Beobachtungszeitpunkt zu bestimmen, welche, mit der im vorherigen Abschnitt konstruierten multidimensionalen Dichte, durch numerische Integration über Hyperwürfel berechnet werden. ${ }^{20}$ Eine analytische Lösung des Optimierungsproblems bzw. der optimalen Asset Allokation kann nicht bestimmt werden. In dieser Studie wird daher ein nichtlinearer Optimierer basierend auf einem Newton-Algorithmus ${ }^{21}$ verwendet.

Um zusätzlich den Einfluss und die Wirkung von unterschiedlichen Anlagemöglichkeiten zu untersuchen, werden zwei Anlageuniversen betrachtet.

20 R-Paket: cubature.

21 R-Funktion: nlm. Als Initial Portfolio für die Optimierung (Startwert) wird das gleichgewichtete Portfolio $(1 / \mathrm{N})$ gewählt. 
$\mathbf{N}=3$ Der Investor hat die Möglichkeit, in drei verschiedene Anlageklassen zu investieren: in zwei riskante - eine langfristige Anleihe und einen Aktienindex - und eine risikofreie Anlage.

$\mathbf{N}=4$ Der Investor hat gegenüber dem Anlageuniversum N=3 die Möglichkeit, zusätzlich in die Rohstoffklasse zu investieren.

Die Bestimmung der Portfoliogewichte erfolgt zu jedem Stichtag eines Monats erneut, indem die obigen Prozesse unter Berücksichtigung der neuen Verteilungsparameter für jeden Beobachtungszeitpunkt wiederholt werden.

\subsubsection{Performance-Kennzahlen}

Für die Beurteilung und Auswertung der Performance der strategischen Asset Allokation mit impliziten Verteilungen werden für den out-of-sample Zeitraum nachfolgend die Kennzahlen Excess Return, realisierte Volatilität und Turnover sowie als zentrale Größe das Sicherheitsäquivalent eingeführt.

(i) Excess Return $\hat{\mu}$

Die Berechnung des Excess Returns erfolgt, indem zu jedem Beobachtungszeitpunkt die realisierte out-of-sample Rendite der untersuchten Strategie bestimmt und um die Rendite der entsprechenden risikofreien Anlage der Periode verringert wird. Der Mittelwert der erhaltenen Zeitreihe wird mit $\hat{\mu}$ bezeichnet.

(ii) Realisierte Volatilität $\hat{\sigma}$

Nach Bestimmung der optimalen Gewichte zu Beginn des Anlagehorizonts wird die Entwicklung des Portfolios auf Basis von Tagesrenditen verfolgt. Aus dieser Wertentwicklung wird die Standardabweichung geschätzt. Diese entspricht der realisierten Volatilität eines bestimmten Anlagehorizonts. Der Mittelwert aus der erhaltenen Zeitreihe entspricht der realisierten Volatilität $\hat{\sigma} .{ }^{22}$

(iii) Sicherheitsäquivalent $\widehat{C E Q}$

Das Sicherheitsäquivalent einer Investitionsstrategie ist der spezifische Zinssatz, bei welchem der Investor indifferent zwischen risikofreier Anlage (zu diesem Zinssatz) und der entsprechenden Strategie ist.

22 Die realisierte Volatilität entpricht zwar nicht der Standardabweichung der Excess Returns, kann jedoch aus Gründen der Interpretation als Vertreter dieser herangezogen werden. Die realisierte Volatilität ist der Standardabweichung der Excess Returns vorzuziehen, da sie eine genauere Schätzung der tatsächlichen Volatilität liefert und zugleich für Signifikanztests verwendet werden kann. 
Für die Ermittlung des Sicherheitsäquivalentes werden aus der Zeitreihe der realisierten Excess Returns $r_{t}$ die Nutzen gemäß der individuellen Nutzenfunktion des Investors mit Risikoaversion $\gamma$ bestimmt. Der Mittelwert hieraus ist dem Nutzen des Sicherheitsäquivalents gleichzusetzen

$$
\frac{(1+\widehat{C E Q})^{1-\gamma}-1}{1-\gamma}=\frac{\frac{1}{141} \sum_{t=1}^{141}\left(1+r_{t}\right)^{1-\gamma}-1}{1-\gamma}
$$

Die nach $\widehat{C E Q}$ umgestellte Gleichung wird als Sicherheitsäquivalent bezeichnet. Falls das Sicherheitsäquivalent der Excess Returns positiv ist, kann die Strategie für den Investor als lohnenswert bzw. nutzenbringend interpretiert werden. Negative Werte hingegen sind als unvorteilhaft bzw. nutzenschmälernd gegenüber der risikofreien Anlage zu verstehen.

(iv) Turnover TO

Um Transaktionskosten für die Umschichtung an den Beobachtungszeitpunkten der jeweiligen Strategie zu berücksichtigen, wird das Turnover ${ }^{23}$ bestimmt. Hierzu werden an jedem Beobachtungszeitpunkt die Gewichte des ursprünglichen Portfolios bestimmt, jeweils mit dem neuen Gewicht für den Anlagezeitraum verglichen und der Absolutbetrag aus der Differenz beider gebildet. Die Summe über alle $N$ Assetklassen ergibt die Umschichtung in einem Zeitpunkt. Der Mittelwert aus der erhaltenen Zeitreihe entspricht dem Turnover der Strategie ${ }^{24}$

$$
\mathrm{TO}=\frac{1}{140} \sum_{i=2}^{141} \sum_{j=1}^{N}\left|\omega_{j, t=i}-\omega_{j, t^{+}=i-1}\right| .
$$

Des Weiteren können mit Hilfe des Turnovers auch um Transaktionskosten bereinigte Excess Returns und Sicherheitsäquivalente bestimmt werden. Hierzu wird die realisierte Rendite zusätzlich verringert, und zwar um den entsprechenden Turnover des Beobachtungszeitpunkts multipliziert mit einem vorgegeben Transaktionskostensatz. Das vorstehend eingeführte Sicherheitsäquivalent wird dementsprechend aus der Zeitreihe bestimmt. Der Transaktionskostensatz wird in dieser Studie mit 10 Basispunkten angesetzt. ${ }^{25}$

$23 \quad$ Vgl. DeMiguel, Garlappi und Uppal (2009b).

24 + bedeutet in diesem Fall "am Ende des Anlagehorizonts". Der Turnover liegt zwischen 0 und 2.

25 Vgl. Plyakha, Uppal und Vilkov (2012), Kempf, Korn und Saßning (2012) und die darin angegebene Literatur. 
Tabelle 3.5: Sicherheitsäquivalente, Excess Returns und realisierte Volatilitäten für die riskanten Anlageklassen

\begin{tabular}{lcccccc}
\hline & $\mathrm{RRA}=2$ & $\mathrm{RRA}=4$ & $\mathrm{RRA}=6$ & $\mathrm{RRA}=8$ & $\hat{\mu}$ & $\hat{\sigma}$ \\
\hline $\mathrm{N}=1$ & & & & & & \\
\hline S\&P 500 & $-3,55 \%$ & $-7,24 \%$ & $-11,05 \%$ & $-15,01 \%$ & $0,03 \%$ & $32,23 \%$ \\
TNX & $-1,79 \%$ & $-2,69 \%$ & $-3,58 \%$ & $-4,46 \%$ & $-0,86 \%$ & $17,86 \%$ \\
$\mathrm{XAU}$ & $0,06 \%$ & $-13,18 \%$ & $-26,70 \%$ & $-40,61 \%$ & $13,35 \%$ & $69,29 \%$ \\
\hline
\end{tabular}

Diese Tabelle gibt die Sicherheitsäquivalente, die Excess Returns und die realisierten Volatilitäten für die riskanten Anlageklassen für Investoren mit CRRA Nutzenfunktionen an. Alle Werte sind annualisiert. Der out-of-sample Zeitraum beträgt Februar 1999 bis Oktober 2010.

Tabelle 3.5 zeigt die Auswertung der Performance-Kennzahlen für die riskanten Anlageklassen. Es zeigt sich, dass eine alleinige Investition in den S\&P 500 Index oder in den TNX Index für keinen der betrachteten Investoren eine erwartete lohnende Investition darstellt. Für den XAU Index bestätigt sich bis auf den Investor mit der Risikoaversion 2 diese Beobachtung. Dieses Resultat ist im Einklang mit der Zeitreihe aus Abbildung 3.1, in welcher beobachtet wurde, dass die kumulierte Anlage in den S\&P 500 und den TNX Index über den betrachteten Zeitraum Vermögen im Vergleich zur risikofreien Anlage vernichtet. Für den XAU Index wird ein hoher Excess Return (13,35 \% p.a.) und eine hohe realisierte Volatilität (69,29 \% p.a.) erzielt. Dies erklärt die entsprechenden Sicherheitsäquivalente der Investoren. Der risikofreudigste Investor $(\mathrm{RRA}=2)$ "würdigt" den hohen Excess Return, dem risikoaversesten Investor $(\mathrm{RRA}=8)$ hingegen "missfällt" die realisierte Volatilität.

\subsubsection{Benchmark Strategien}

Zur Vergleichbarkeit und Beurteilung der Performance der strategischen Asset Allokation mit impliziten Informationen werden zum einen aktive Strategien auf Basis historischer Renditezeitreihen, zum anderen passive Anlagestrategien betrachtet.

Die optimale Asset Allokation auf Basis historischer Daten erfolgt analog zu dem Fall impliziter Informationen, indem der Erwartungswert und die Matrizen $M_{2}, M_{3}, M_{4}$ aus historischen Daten gewonnen werden. Hierzu werden zu jedem Zeitpunkt rückblickend aus den Beobachtungen der letzten 36, 60 und 120 Monate für jedes riskante Asset Verteilungsparameter der Schiefen-Normalverteilung mit Hilfe eines Maximum-Likelihood An- 
satzes $^{26}$ geschätzt. ${ }^{27}$ Für die Modellierung der Abhängigkeitsstruktur unter den riskanten Assets wird dieselbe Copula wie zuvor bei den impliziten Verteilungen bemüht. Dies ist darin begründet, dass bei der Auswertung der out-of-sample Performance die Wahl der Abhängigkeitsstruktur somit als Ergebnistreiber ausgeschlossen werden kann. Der Erwartungswert und die Matrizen $M_{2}, M_{3}, M_{4}$ können schließlich wiederum mittels numerischer Integration über Hyperwürfel bestimmt werden.

Des Weiteren werden fünf passive Anlagestrategien ausgewertet. Zum einen eine $1 / N$ Strategie, welche das Vermögen gleichmäßig auf die verfügbaren Assetklassen verteilt. Zum anderen vier Strategien, welche jeweils einen festen Anteil in die risikofreie Anlage investieren und den übrigen Anteil des Vermögens gleichmäßig auf die verfügbaren riskanten Anlagewerte verteilen. Am Ende eines jeden Anlagehorizonts werden die Anteile in den Anlageklassen im Portfolio für die passiven Strategien neu berechnet und zur ursprünglichen Gewichtung wieder umgeschichtet. Dies bedeutet, dass die passiven Strategien auch Transaktionskosten verursachen.

Tabelle 3.6 zeigt einen Überblick der untersuchten Anlagestrategien im Rahmen der strategischen Asset Allokation dieser Studie.

Tabelle 3.6: Übersicht der betrachteten aktiven und passiven Strategien der empirischen Studie

\begin{tabular}{|c|c|c|c|c|c|c|c|}
\hline & $R_{f}$ & S\&P 500 & TNX & $R_{f}$ & S\&P 500 & TNX & $\mathrm{XAU}$ \\
\hline Aktiv & \multicolumn{3}{|c|}{$\mathrm{N}=3$} & \multicolumn{4}{|c|}{$\mathrm{N}=4$} \\
\hline $36 \mathrm{M}$ & \multirow{4}{*}{\multicolumn{7}{|c|}{$\begin{array}{l}\text { historische Renditen mit Schätzhorizont } 36 \text { Monate } \\
\text { historische Renditen mit Schätzhorizont } 60 \text { Monate } \\
\text { historische Renditen mit Schätzhorizont } 120 \text { Monate } \\
\text { implizite Informationen aus Optionsmarktdaten }\end{array}$}} \\
\hline $60 \mathrm{M}$ & & & & & & & \\
\hline $120 \mathrm{M}$ & & & & & & & \\
\hline Implizit & & & & & & & \\
\hline Passiv & \multicolumn{3}{|c|}{$\mathrm{N}=3$} & \multicolumn{4}{|c|}{$\mathrm{N}=4$} \\
\hline $1 / \mathrm{N}$ & $33,3 \%$ & $33,3 \%$ & $33,3 \%$ & $25,0 \%$ & $25,0 \%$ & $25,0 \%$ & $25,0 \%$ \\
\hline $\mathrm{Rf}=0 \%$ & $0,0 \%$ & $50,0 \%$ & $50,0 \%$ & $0,0 \%$ & $33,3 \%$ & $33,3 \%$ & $33,3 \%$ \\
\hline $\mathrm{Rf}=25 \%$ & $25,0 \%$ & $37,5 \%$ & $37,5 \%$ & $25,0 \%$ & $25,0 \%$ & $25,0 \%$ & $25,0 \%$ \\
\hline $\mathrm{Rf}=50 \%$ & $50,0 \%$ & $25,0 \%$ & $25,0 \%$ & $50,0 \%$ & $16,6 \%$ & $16,6 \%$ & $16,6 \%$ \\
\hline $\mathrm{Rf}=75 \%$ & $75,0 \%$ & $12,5 \%$ & $12,5 \%$ & $75,0 \%$ & $8,3 \%$ & $8,3 \%$ & $8,3 \%$ \\
\hline
\end{tabular}

Diese Tabelle zeigt eine Übersicht aller betrachteten aktiven und passiven Strategien der empirischen Studie. $\mathrm{Rf}=\cdot \%$ gibt für die entsprechende passive Strategie den Anteil in der risikofreien Anlage an.

26 R-Paket: SN.

27 Die Schiefe-Normalverteilung wird in der Portfolioselektion mit höheren Momente insbesondere in Harvey, Liechty, Liechty und Muller (2010) propagiert und bedeuted im Rahmen dieser Studie, dass die Randverteilungen der riskanten Assets schief-normalverteilt sind. Daher unterstellen die historischen Strategien weder symmetrische Randverteilungen noch eine Kurtosis von drei. 


\subsection{Ergebnisse der empirischen Studie}

Dieser Abschnitt stellt zunächst die Ergebnisse der Performance-Kennzahlen für die Anlagestrategien aus Tabelle 3.6 vor. Die weiteren Analysen konzentrieren sich auf die zentralen Forschungsfragen zur Vorteilhaftigkeit impliziter Verfahren und der Wirkung höherer Momente. Des Weiteren wird die Robustheit der Ergebnisse bei zusätzlicher Betrachtung von Transaktionskosten untersucht.

Tabelle 3.7 zeigt die Ergebnisse für die aktiven Strategien sowie für die passive 1/N Strategie. Im Vergleich zu einem reinen Investment in nur eine einzelne riskante Anlageklasse (vgl. Tabelle 3.5) wird an der 1/N Strategie für beide Anlageuniversen die Vorteilhaftigkeit der Portfoliobildung und somit der Diversifizierung des Vermögens auf unterschiedliche Anlagewerte deutlich. Jedoch liefern die aktiven ebenso wie die passive Strategie für das Anlageuniversum N=3 ausschließlich negative erwartete Sicherheitsäquivalente und stellen daher gegenüber der risikofreien Anlage eine nicht lohnenswerte Investition dar. Diese Beobachtung ist gleichsam für das erweiterte Anlageuniversum $\mathrm{N}=4$ gültig, sofern von der impliziten Strategie des Investors mit Risikoaversion 2 und dem passiven 1/N Benchmark für die Risikoaversionen 2 und 4 abgesehen wird.

Die passive $1 / \mathrm{N}$ Strategie kann weder für das Anlageuniversum $\mathrm{N}=3$ noch für das um Rohstoffe erweiterte Universum durch eine aktive historische Strategie (36M, 60M und $120 \mathrm{M}$ ) in Bezug auf das Sicherheitsäquivalent geschlagen werden. Dies ist insbesondere auf die schlechteren realisierten Excess Returns zurückzuführen und im Einklang mit den Beobachtungen aus den Studien von DeMiguel, Garlappi und Uppal (2009b) sowie Fugazza, Guidolin und Nicodano (2010). Für die impliziten Strategien wird ein ähnliches Ergebnis beobachtet. Eine Ausnahme stellt der Investor mit Risikoaversion 8 im Rahmen des Anlageuniversums $\mathrm{N}=3$ dar, in diesem Fall gilt, dass das Sicherheitsäquivalent der impliziten Strategie höher als das des passiven 1/N Benchmarks ist. Dieses Ergebnis basiert auf einer deutlich geringeren realisierten Volatilität.

In Bezug auf die Gegenüberstellung der Strategien auf Basis historischer und impliziter Informationen zeigen Kostakis, Panigirtzoglou und Skiadopoulos (2011), dass der implizite Ansatz historischen Benchmark Strategien im Sinne der Sharpe Ratio vorzuziehen sind. Dieses Resultat kann in der vorliegenden Studie hinsichtlich des Sicherheitsäquivalentes für alle Investoren bestätigt werden. Das verbesserte Sicherheitsäquivalent ist insbesondere bis auf den Fall $\mathrm{N}=3$ und Risikoaversion 2 auf die signifikant geringeren realisierten Volatilitäten der impliziten Strategien zurückzuführen. Die Excess Returns hingegen weisen keine signifikante Out-Performance auf. 


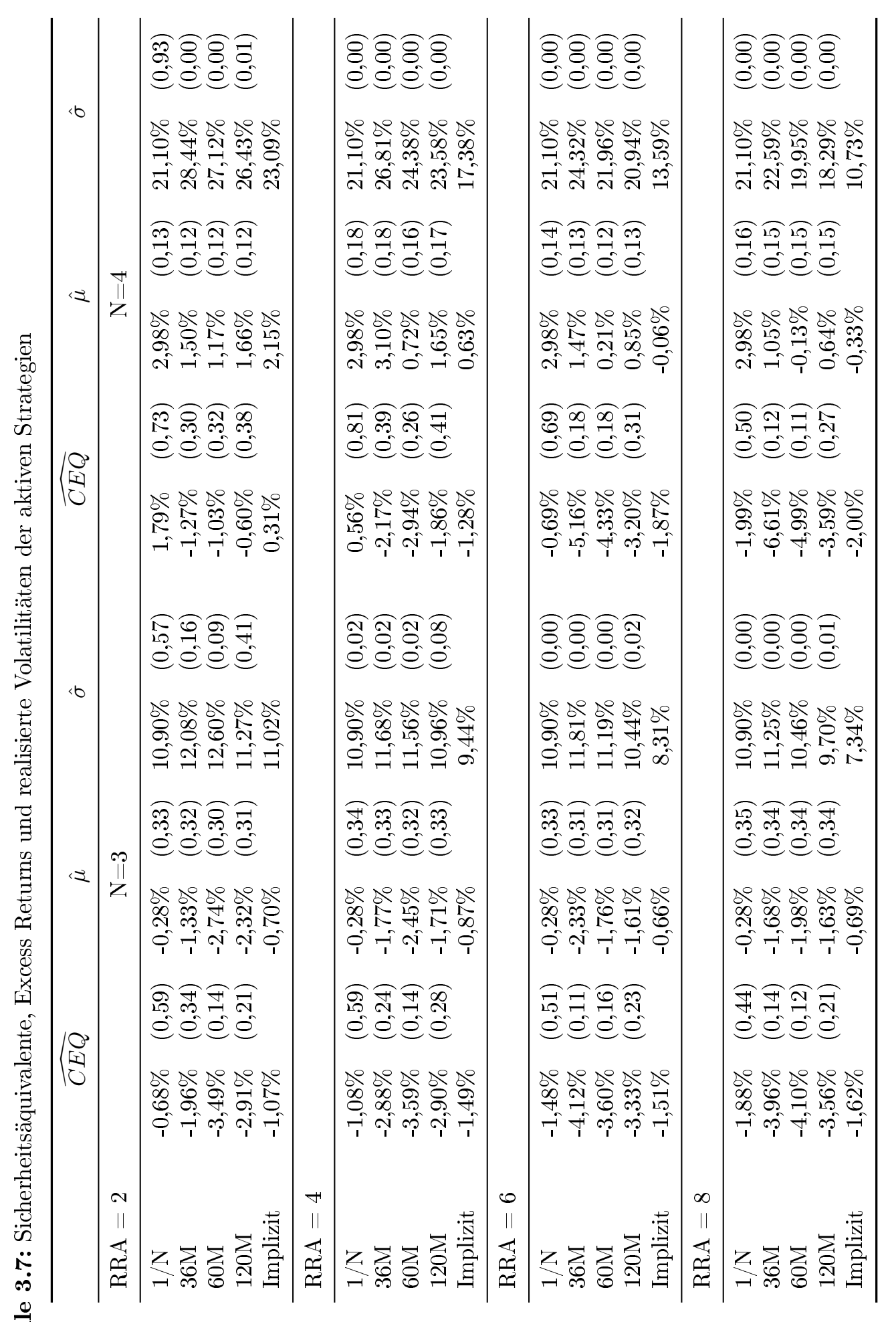

苯若离

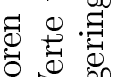

范然

至

进需容

वृ

党

专的

표용

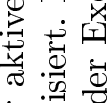

ษ)

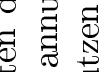

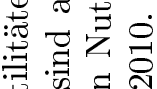

क्ष

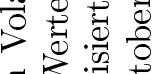

ब

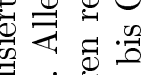

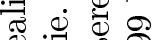

额:

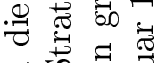

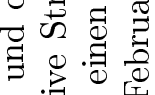

吅

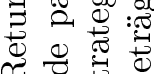

क क क

记

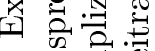

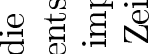

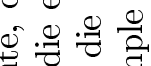

政

定

垴

눙

过

긍

语 छ

$\because$.

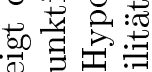

\&

呵

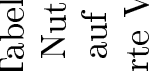

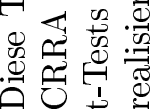


Ein Vergleich zwischen den Anlageuniversen erlaubt die Beurteilung des Nutzens einer Rohstoffklasse im Asset-Mix. Für die aktiven Strategien ergibt sich ein differenziertes Bild bzgl. der Vorteilhaftigkeit. Risikofreudigere Investoren (RRA 2 und 4) profitieren von der Hinzunahme, für risikoaversere Investoren (RRA 6 und 8) sinkt das Sicherheitsäquivalent. Diese Beobachtung kann mit einer analogen Argumentation wie beim XAU Index erläutert werden. So ergibt sich beispielhaft für die implizite Strategie, dass Investoren mit Risikoaversion 2 (und 4) den höheren Excess Return ihrer Anlage ( $\mathrm{N}=3$ : -0,70 \% (-0,87 \%) p.a. vs. $\mathrm{N}=4: 2,15 \%(0,63 \%)$ p.a.) "würdigen", die risikoaverseren Investoren 6 (und 8) hingegen die geringere realisierte Volatilität $(\mathrm{N}=3: 8,31 \%(7,34 \%)$ p.a. vs. $\mathrm{N}=4$ : $13,59 \%(10,73 \%)$ p.a.) schätzen.

Zusammenfassend liefert die passive 1/N Strategie das höchste Sicherheitsäquivalent für alle Investoren. Dennoch wird für keinen betrachteten Fall die implizite Strategie statistisch signifikant durch die passive 1/N Strategie geschlagen. Diese Erkenntnis lässt sich auch für die erzielten Excess Returns beobachten. Im Sinne der realisierten Volatilitäten zeigen die impliziten Strategien außer für den Investor mit der Risikoaversion 2 statistisch signifikant geringere realisierte Volatilitäten im Vergleich zu den historischen Benchmark Strategien und zu der passiven 1/N Strategie.

Tabelle 3.8: Sicherheitsäquivalente, Excess Returns und realisierte Volatilitäten der passiven Strategien

\begin{tabular}{lcccccc}
\hline & $\mathrm{RRA}=2$ & $\mathrm{RRA}=4$ & $\mathrm{RRA}=6$ & $\mathrm{RRA}=8$ & $\hat{\mu}$ & $\hat{\sigma}$ \\
\hline $\mathrm{N}=3$ & & & & & & \\
\hline $\mathrm{Rf}=0 \%$ & $-1,31 \%$ & $-2,21 \%$ & $-3,11 \%$ & $-4,01 \%$ & $-0,42 \%$ & $16,34 \%$ \\
$\mathrm{Rf}=25 \%$ & $-0,82 \%$ & $-1,32 \%$ & $-1,83 \%$ & $-2,34 \%$ & $-0,31 \%$ & $12,26 \%$ \\
$\mathrm{Rf}=50 \%$ & $-0,43 \%$ & $-0,66 \%$ & $-0,88 \%$ & $-1,11 \%$ & $-0,21 \%$ & $8,17 \%$ \\
$\mathrm{Rf}=75 \%$ & $-0,16 \%$ & $-0,22 \%$ & $-0,27 \%$ & $-0,33 \%$ & $-0,10 \%$ & $4,09 \%$ \\
\hline $\mathrm{N}=4$ & & & & & & \\
\hline $\mathrm{Rf}=0 \%$ & $1,84 \%$ & $-0,38 \%$ & $-2,69 \%$ & $-5,13 \%$ & $3,99 \%$ & $28,13 \%$ \\
$\mathrm{Rf}=25 \%$ & $1,79 \%$ & $0,56 \%$ & $-0,69 \%$ & $-1,99 \%$ & $2,98 \%$ & $21,10 \%$ \\
$\mathrm{Rf}=50 \%$ & $1,45 \%$ & $0,92 \%$ & $0,38 \%$ & $-0,17 \%$ & $1,98 \%$ & $14,07 \%$ \\
$\mathrm{Rf}=75 \%$ & $0,86 \%$ & $0,73 \%$ & $0,59 \%$ & $0,46 \%$ & $0,98 \%$ & $7,03 \%$ \\
\hline
\end{tabular}

Diese Tabelle zeigt die Sicherheitsäquivalente, die Excess Returns und die realisierten Volatilitäten der passiven Strategien für Investoren mit CRRA Nutzenfunktionen. Rf $=$. \% gibt für die entsprechende passive Strategie den Anteil in der risikofreien Anlage an. Alle Werte sind annualisiert. Der out-of-sample Zeitraum beträgt Februar 1999 bis Oktober 2010.

Tabelle 3.8 zeigt für die weiteren passiven Strategien die Auswertung der PerformanceKennzahlen. Es ist zu beobachten, dass ein höherer Anteil in der risikofreien Anlage zu einer Konvergenz von Excess Return und realisierter Volatilität gegen $0 \%$ führt. Dies ist darin begründet, dass zum einen der realisierte Return nur durch in einen kleineren Anteil 
im Portfolio von der risikofreien Anlage abweicht, zum anderen der Anteil an riskanten Assets die realisierte Volatilität determiniert.

Im Anlageuniversum $\mathrm{N}=3$ haben die passiven Strategien für alle betrachteten Investoren negative Sicherheitsäquivalente. Dennoch steigen die entsprechenden Werte im Vergleich zu den reinen Investments in Aktien oder langfristige Anleihen aus Tabelle 3.5 für alle Investoren deutlich. Dies lässt sich insbesondere am Fall Rf $=0 \%$ erläutern. Diese Anlagestrategie besteht ausschließlich aus riskanten Assets und zeigt somit gleichsam mögliche Diversifikationspotentiale unter den Bestandteilen auf. Die mittlere realisierte Volatilität dieser Strategie (16,34 \% p.a.) ist wie vermutet geringer als die ihrer einzelnen Bestandteile - Aktienindex (32,23 \% p.a.) und langfristige Anleihen (17,86\% p.a.) - und hat somit einen positiven Einfluss auf die Sicherheitsäquivalente der Investoren.

Die Hinzunahme von Rohstoffen in das Anlageuniversum ( $\mathrm{N}=4)$ wiederum erhöht für alle betrachteten Strategien den Excess Return sowie die mittlere realisierte Volatilität. Die Ergebnisse zeigen, dass - außer im Fall von Rf $=0 \%$ und der Risikoaversion 8 die Hinzunahme von Rohstoffen von Vorteil ist. Im Vergleich zu den zuvor diskutierten aktiven Strategien ist insbesondere festzuhalten, dass die $\mathrm{Rf}=75 \%$ - Strategie als einzige für alle betrachteten Investoren einen positives Sicherheitsäquivalent zeigt und daher als lohnenswert gegenüber der risikofreien Anlage eingestuft werden kann. 


\subsubsection{Gründe für die Out-Performance der impliziten Strategie gegenüber den historischen Benchmark Strategien}

Tabelle 3.9: Sicherheitsäquivalente, Excess Returns und realisierte Volatilitäten der aktiven historischen Strategien mit impliziten Erwartungswerten

\begin{tabular}{lcccccc}
\hline & $\widehat{C E Q}$ & $\hat{\mu}$ & $\hat{\sigma}$ & $\widehat{C E Q}$ & $\hat{\mu}$ & $\hat{\sigma}$ \\
\hline $\mathrm{RRA}=2$ & & $\mathrm{~N}=3$ & & & $\mathrm{~N}=4$ & \\
\hline $36 \mathrm{M}$ & $-2,35 \%$ & $-1,73 \%$ & $12,73 \%$ & $0,05 \%$ & $1,96 \%$ & $24,50 \%$ \\
$60 \mathrm{M}$ & $-1,34 \%$ & $-0,81 \%$ & $12,52 \%$ & $-1,01 \%$ & $1,16 \%$ & $25,77 \%$ \\
$120 \mathrm{M}$ & $-1,06 \%$ & $-0,57 \%$ & $12,32 \%$ & $-0,58 \%$ & $1,58 \%$ & $25,97 \%$ \\
\hline $\mathrm{RRA}=4$ & & & & & & \\
\hline $36 \mathrm{M}$ & $-2,25 \%$ & $-1,16 \%$ & $12,58 \%$ & $-2,33 \%$ & $0,96 \%$ & $21,98 \%$ \\
$60 \mathrm{M}$ & $-2,94 \%$ & $-1,83 \%$ & $11,95 \%$ & $-2,34 \%$ & $1,03 \%$ & $22,51 \%$ \\
$120 \mathrm{M}$ & $-1,37 \%$ & $-0,51 \%$ & $11,29 \%$ & $-1,45 \%$ & $1,64 \%$ & $22,06 \%$ \\
\hline $\mathrm{RRA}=6$ & & & & & & \\
\hline $36 \mathrm{M}$ & $-2,85 \%$ & $-1,26 \%$ & $12,25 \%$ & $-3,19 \%$ & $0,31 \%$ & $19,20 \%$ \\
$60 \mathrm{M}$ & $-2,86 \%$ & $-1,46 \%$ & $11,20 \%$ & $-3,14 \%$ & $0,44 \%$ & $19,06 \%$ \\
$120 \mathrm{M}$ & $-2,25 \%$ & $-1,12 \%$ & $10,62 \%$ & $-3,21 \%$ & $0,00 \%$ & $18,44 \%$ \\
\hline $\mathrm{RRA}=8$ & & & & & & \\
\hline $36 \mathrm{M}$ & $-2,88 \%$ & $-0,94 \%$ & $11,68 \%$ & $-4,11 \%$ & $-0,28 \%$ & $17,22 \%$ \\
$60 \mathrm{M}$ & $-2,96 \%$ & $-1,22 \%$ & $10,80 \%$ & $-4,60 \%$ & $-0,86 \%$ & $16,65 \%$ \\
$120 \mathrm{M}$ & $-2,55 \%$ & $-0,99 \%$ & $10,28 \%$ & $-3,54 \%$ & $-0,39 \%$ & $15,91 \%$ \\
\hline
\end{tabular}

Diese Tabelle zeigt die Sicherheitsäquivalente, die Excess Returns und die realisierten Volatilitäten der aktiven historischen Strategien mit impliziten Erwartungswerten für Investoren mit CRRA Nutzenfunktionen. Alle Werte sind annualisiert. Der out-of-sample Zeitraum beträgt Februar 1999 bis Oktober 2010.

Die Resultate aus Tabelle 3.7 zeigen eine Vorteilhaftigkeit der impliziten Strategien gegenüber den historischen Benchmark Strategien im Sinne des Sicherheitsäquivalentes. In diesem Zusammenhang identifizieren Kostakis, Panigirtzoglou und Skiadopoulos (2011) eine verbesserte Schätzung des Erwartungswertes durch die implizite Methodik als Grund für die bessere Performance.

Tabelle 3.9 zeigt die Ergebnisse für die historischen Strategien, wobei der Erwartungswert durch den aus der impliziten Strategie geschätzten Wert ersetzt wurde. Im Vergleich zu den Resultaten aus Tabelle 3.7 zeigt sich, dass sich die Vorteilhaftigkeit impliziter Strategien insbesondere für den Fall eines risikoaverseren Investors bestätigen lässt. Dabei ist $\mathrm{zu}$ beobachten, dass für das Anlageuniversum $\mathrm{N}=3$ vor allem die Excess Returns, für das erweiterte Anlageuniversum $\mathrm{N}=4$ die realisierte Volatilität von der impliziten Schätzung des Erwartungswertes profitieren. Da der Erwartungswert aus rein historischen 
Daten als schwierig zu schätzen gilt (vgl. z. B. Merton (1980)), die Asset Allokation jedoch sehr sensitiv gegenüber diesem Wert ist,${ }^{28}$ übersetzt sich eine verbesserte Schätzung unmittelbar in die out-of-sample Performance.

In der Diskussion der Ergebnisse von Tabelle 3.7 wurde festgestellt, dass sich die impliziten Strategien gegenüber den historischen Benchmarks in den überwiegenden Fällen durch eine signifikant geringere realisierte Volatilität auszeichnen. Aus der empirischen Forschung ist bekannt, dass implizite Verfahren insbesondere eine verbesserte Prognose zukünftiger Volatilitäten gegenüber klassischen Methoden gewährleisten. ${ }^{29}$ In der Asset Allokation führt dies zu einer unterschiedlichen Gewichtung riskanter Assets bzw. im Fall der Möglichkeit der Anlage in ein risikofreies Asset zu einer veränderten Gewichtung dieses Titels. Letztere hat unmittelbaren Einfluss auf die realisierten Volatilitäten, da diese auschließlich durch den riskanten Anteil im Portfolio determiniert wird und somit ein höheres Gewicht der risikofreien Anlage zu einer Reduktion der realisierten Volatilität führt.

Tabelle 3.10: Deskriptive Statistiken für das Gewicht der risikofreien Anlage

\begin{tabular}{lcccccc}
\hline & ERW & STD & MED & ERW & STD & MED \\
\hline $\mathrm{N}=3$ & \multicolumn{3}{c}{ RRA $=4$} & \multicolumn{3}{c}{ RRA $=8$} \\
\hline $36 \mathrm{M}$ & $50,8 \%$ & $34,8 \%$ & $58,1 \%$ & $54,5 \%$ & $33,7 \%$ & $62,5 \%$ \\
$60 \mathrm{M}$ & $50,4 \%$ & $38,4 \%$ & $57,4 \%$ & $57,2 \%$ & $33,9 \%$ & $68,7 \%$ \\
$120 \mathrm{M}$ & $60,8 \%$ & $25,5 \%$ & $73,4 \%$ & $65,2 \%$ & $24,1 \%$ & $78,4 \%$ \\
Implizit & $53,0 \%$ & $31,7 \%$ & $63,0 \%$ & $63,9 \%$ & $27,6 \%$ & $70,7 \%$ \\
\hline $\mathrm{N}=4$ & \multicolumn{3}{c}{$\mathrm{RRA}=4$} & \multicolumn{3}{c}{$\mathrm{RRA}=8$} \\
\hline $36 \mathrm{M}$ & $32,8 \%$ & $24,6 \%$ & $40,2 \%$ & $40,2 \%$ & $23,8 \%$ & $45,4 \%$ \\
$60 \mathrm{M}$ & $35,6 \%$ & $22,9 \%$ & $41,2 \%$ & $45,1 \%$ & $20,3 \%$ & $46,2 \%$ \\
$120 \mathrm{M}$ & $41,2 \%$ & $19,5 \%$ & $43,1 \%$ & $52,5 \%$ & $21,7 \%$ & $54,8 \%$ \\
Implizit & $44,6 \%$ & $28,8 \%$ & $52,5 \%$ & $59,7 \%$ & $26,8 \%$ & $69,5 \%$ \\
\hline
\end{tabular}

Diese Tabelle zeigt für die aktiven Strategien von Investoren mit CRRA Nutzenfunktionen für die Risikoaversionen 4 und 8 deskriptive Statistiken für das Gewicht in der risikofreien Anlage. Der out-of-sample Zeitraum beträgt Februar 1999 bis Oktober 2010.

Tabelle 3.10 zeigt für die betrachteten Strategien deskriptive Statistiken für das Gewicht in der risikofreien Anlage für Investoren mit Risikoaversionen 4 und 8. Für alle betrachteten Fälle ist zu beobachten, dass für einen risikoaverseren Investor (Vergleich $R R A=4$ mit $\mathrm{RRA}=8$ ) das Gewicht im Mittel steigt und die Standardabweichung sinkt. Dies ist im Einklang mit dem in Kapitel 2 diskutierten Beispiel, in welchem gezeigt wurde, dass sich die Risikoaversion auch im Gewicht der risikofreien Anlage widerspiegelt und eine höhere Risikoaversion zu einem höheren Anteil der risikofreien Anlage führt.

28 Aus diesem Grund liefert das globale Minimum-Varianz-Portfolio in vielen Portfoliostudien eine bessere out-of-sample Performance als das optimale Tangentialportfolio (vgl. z. B. DeMiguel, Garlappi und Uppal (2009b)).

29 Vgl. Motivation in der Einleitung. 
Des Weiteren ist zu erkennen, dass wie vermutet das Gewicht in der risikofreien Anlage für die implizite Strategie im Mittel am höchsten ist. Lediglich für die historische 120M Strategie und Risikoaversion 4 ist das Gewicht in der risikofreien Anlage höher. Dies ist auch im Einklang mit dem Ergebnis aus Tabelle 3.7, dass für diesen Fall die Hypothese einer geringeren Volatilität der impliziten Strategie nicht auf dem 5\%-Niveau verworfen werden kann. Für das erweiterte Anlageuniversum $\mathrm{N}=4$ ist weiter zu beobachten, dass für die impliziten Strategien für beide Risikoaversionen das Gewicht deutlich stärker variiert als das entsprechende Gewicht für die historische Benchmark Strategie, was einen höheren Turnover vermuten lässt.

\subsubsection{Analyse der Komposition des riskanten Anteils der aktiven Strategien in der Asset Allokation}

In der klassischen Portfoliotheorie nach Markowitz (1952) lässt sich die Asset Allokation durch die Aufteilung des Vermögens auf die risikofreie Anlage und das Tangentialportfolio beschreiben. Dies wird als Two-Fund Separation oder Tobin Separation bezeichnet. Investoren mit unterschiedlicher Risikoaversion halten somit dieselbe Komposition an riskanten Assets im Portfolio. Die Risikoaversion steuert lediglich die Höhe des Anteils der risikofreien Anlage bzw. des für alle Investoren gleich zusammengesetzten riskanten Portfolios. Die Separation gilt im Fall von normalverteilten Renditen und Investoren mit CRRA Nutzenfunktionen. ${ }^{30}$

In dieser Studie werden jedoch keine normalverteilten Renditen unterstellt und die höhere Momente Schiefe und Kurtosis in das Nutzenkalkül mit einbezogen. Daher kann die Komposition des riskanten Anteils des Portfolios für Investoren mit unterschiedlichen Risikoaversionen voneinander abweichen. Ob dies empirisch zutrifft, wird folgend untersucht.

30 Vgl. Jurek und Viceira (2011). 
Tabelle 3.11: Durchschnittliche Komposition des riskanten Anteils der Asset Allokation

\begin{tabular}{lrrrrrr}
\hline & S\&P500 & TNX & XAU & S\&P500 & TNX & XAU \\
\hline $\mathrm{N}=3$ & \multicolumn{3}{r}{ RRA=4 } & & $\mathrm{RRA}=8$ & \\
\hline $36 \mathrm{M}$ & $60,2 \%$ & $39,8 \%$ & & $60,9 \%$ & $39,1 \%$ & \\
$60 \mathrm{M}$ & $63,9 \%$ & $36,1 \%$ & & $65,0 \%$ & $35,0 \%$ & \\
$120 \mathrm{M}$ & $77,8 \%$ & $22,2 \%$ & & $78,4 \%$ & $21,6 \%$ & \\
Implizit & $54,5 \%$ & $45,5 \%$ & & $52,9 \%$ & $47,1 \%$ & \\
\hline $\mathrm{N}=4$ & \multicolumn{3}{r}{$\mathrm{RRA}=4$} & \multicolumn{3}{r}{$\mathrm{RRA}=8$} \\
\hline $36 \mathrm{M}$ & $24,8 \%$ & $28,8 \%$ & $46,4 \%$ & $25,8 \%$ & $30,2 \%$ & $44,0 \%$ \\
$60 \mathrm{M}$ & $25,3 \%$ & $25,7 \%$ & $49,0 \%$ & $24,7 \%$ & $27,9 \%$ & $47,4 \%$ \\
$120 \mathrm{M}$ & $31,6 \%$ & $13,6 \%$ & $54,8 \%$ & $30,6 \%$ & $14,5 \%$ & $54,9 \%$ \\
Implizit & $24,5 \%$ & $35,8 \%$ & $39,7 \%$ & $27,0 \%$ & $42,2 \%$ & $30,8 \%$ \\
\hline
\end{tabular}

Diese Tabelle zeigt für die aktiven Strategien von Investoren mit CRRA Nutzenfunktionen und Risikoaversionen 4 und 8 die durchschnittliche Komposition des riskanten Anteils in der Asset Allokation. Die entsprechenden Gewichte sind normiert zu eins. Der out-of-sample Zeitraum beträgt Februar 1999 bis Oktober 2010.

Tabelle 3.11 zeigt für die betrachteten Strategien die durchschnittlichen Gewichte in den riskanten Assets. Die Werte sind zur Vergleichbarkeit auf eins normiert und sind somit um den Anteil in der risikofreien Anlage bereinigt dargestellt. Für das Anlageuniversum N=3 lässt sich erkennen, dass die Gewichtung für unterschiedliche Strategien deutlich variiert. Die historische 120M Strategie hält im Vergleich zu der 36M, 60M und impliziten Strategie einen höheren Anteil im S\&P 500. Ein Vergleich der unterschiedlichen Investoren innerhalb derselben Strategie zeigt jedoch nur marginale Unterschiede in der Komposition. Es lässt sich folgern, dass die Risikoaversion wie in der klassischen Portfoliotheorie vor allem in der Aufteilung zwischen riskantem und risikofreiem Anteil im Portfolio zum Ausdruck kommt. Hingegen lassen sich für das um die Rohstoffklasse erweiterte Anlageuniversum deutliche Unterschiede in den Gewichten erkennen. Tabelle 3.12 zeigt hierzu deskriptive Statistiken für die Differenz in den Gewichten von Investoren mit Risikoaversionen 4 und 8 sowie p-Werte auf die Hypothese, dass die Differenz null ist. 
Tabelle 3.12: Deskriptive Statistiken für die Differenz der normierten Gewichte zwischen den Risikoaversionen 4 und 8 der riskanten Assets

\begin{tabular}{|c|c|c|c|c|c|c|c|}
\hline & & ERW & STD & $75 \%$ & MED & $25 \%$ & p-Wert \\
\hline \multicolumn{8}{|l|}{$\mathrm{N}=3$} \\
\hline \multirow[t]{2}{*}{$36 \mathrm{M}$} & $\Delta$-S\&P 500 & $-0,7 \%$ & $13,2 \%$ & $0,0 \%$ & $0,0 \%$ & $0,0 \%$ & 0,5047 \\
\hline & $\Delta$-TNX & $0,7 \%$ & $13,2 \%$ & $0,0 \%$ & $0,0 \%$ & $0,0 \%$ & 0,5047 \\
\hline \multirow[t]{2}{*}{$60 \mathrm{M}$} & $\Delta$-S\&P 500 & $-1,1 \%$ & $13,3 \%$ & $0,0 \%$ & $0,0 \%$ & $0,0 \%$ & 0,3454 \\
\hline & $\Delta$-TNX & $1,1 \%$ & $13,3 \%$ & $0,0 \%$ & $0,0 \%$ & $0,0 \%$ & 0,3454 \\
\hline \multirow[t]{2}{*}{$120 \mathrm{M}$} & $\Delta$-S\&P 500 & $-0,6 \%$ & $9,2 \%$ & $0,0 \%$ & $0,0 \%$ & $0,0 \%$ & 0,4597 \\
\hline & $\Delta$-TNX & $0,6 \%$ & $9,2 \%$ & $0,0 \%$ & $0,0 \%$ & $0,0 \%$ & 0,4597 \\
\hline \multirow[t]{2}{*}{ Implizit } & $\Delta$-S\&P 500 & $1,6 \%$ & $9,8 \%$ & $0,0 \%$ & $0,0 \%$ & $0,0 \%$ & 0,0567 \\
\hline & $\Delta$-TNX & $-1,6 \%$ & $9,8 \%$ & $0,0 \%$ & $0,0 \%$ & $0,0 \%$ & 0,0567 \\
\hline \multicolumn{8}{|l|}{$\mathrm{N}=4$} \\
\hline \multirow[t]{3}{*}{$36 \mathrm{M}$} & $\Delta$-S\&P 500 & $-0,9 \%$ & $9,3 \%$ & $0,5 \%$ & $0,0 \%$ & $-0,1 \%$ & 0,2353 \\
\hline & $\Delta$-TNX & $-1,4 \%$ & $7,1 \%$ & $0,0 \%$ & $-0,7 \%$ & $-3,5 \%$ & 0,0193 \\
\hline & $\Delta-\mathrm{XAU}$ & $2,3 \%$ & $7,5 \%$ & $3,7 \%$ & $1,4 \%$ & $0,0 \%$ & 0,0003 \\
\hline \multirow[t]{3}{*}{$60 \mathrm{M}$} & $\Delta$-S\&P 500 & $0,6 \%$ & $7,5 \%$ & $2,7 \%$ & $0,0 \%$ & $-0,2 \%$ & 0,3273 \\
\hline & $\Delta$-TNX & $-2,2 \%$ & $7,1 \%$ & $0,0 \%$ & $-2,7 \%$ & $-4,5 \%$ & 0,0004 \\
\hline & $\Delta$-XAU & $1,5 \%$ & $7,5 \%$ & $5,1 \%$ & $2,9 \%$ & $0,0 \%$ & 0,0153 \\
\hline \multirow[t]{3}{*}{$120 \mathrm{M}$} & $\Delta$-S\&P 500 & $1,1 \%$ & $8,8 \%$ & $7,4 \%$ & $0,0 \%$ & $-4,1 \%$ & 0,1514 \\
\hline & $\Delta$-TNX & $-0,9 \%$ & $5,7 \%$ & $0,0 \%$ & $0,0 \%$ & $-1,8 \%$ & 0,0556 \\
\hline & $\Delta-\mathrm{XAU}$ & $-0,1 \%$ & $8,7 \%$ & $5,4 \%$ & $3,5 \%$ & $-8,0 \%$ & 0,8521 \\
\hline \multirow[t]{3}{*}{ Implizit } & $\Delta$-S\&P 500 & $-2,5 \%$ & $9,1 \%$ & $0,0 \%$ & $0,0 \%$ & $-4,7 \%$ & 0,0013 \\
\hline & $\Delta-\mathrm{TNX}$ & $-6,4 \%$ & $14,2 \%$ & $0,0 \%$ & $-1,0 \%$ & $-8,1 \%$ & 0,0000 \\
\hline & $\Delta-\mathrm{XAU}$ & $8,9 \%$ & $13,3 \%$ & $11,2 \%$ & $5,2 \%$ & $1,5 \%$ & 0,0000 \\
\hline
\end{tabular}

Diese Tabelle zeigt für die aktiven Strategien von Investoren mit CRRA Nutzenfunktionen und Risikoaversionen 4 und 8 die deskriptive Statistik für die Differenz der normierten Gewichte zwischen den Risikoaversionen im entsprechenden riskanten Asset. Der p-Wert gibt die Auswertung des t-Tests auf die Hypothese, dass der Wert gleich null ist, an. Der out-of-sample Zeitraum beträgt Februar 1999 bis Oktober 2010.

Es lässt sich feststellen, wie bereits an den durchschnittlichen Gewichten aus Tabelle 3.11 beobachtet, dass für $\mathrm{N}=3$ die Differenz für die historischen Strategien nicht signifikant von null verschieden ist. Lediglich für die implizite Strategie ist die Differenz noch auf einem 10\%-Niveau, aber nicht mehr auf einem 5\%-Niveau, signifikant. Dennoch lässt sich an den Quantilen und dem Median erkennen, dass die Verteilung der Differenz um null konzentriert ist.

Für das erweiterte Anlageuniversum lassen sich insbesondere für die implizite Strategie signifikante Differenzen erkennen. In diesem Fall ist ein deutlicher Unterschied im Gewicht des S\&P 500 und des XAU Indexes zu erkennen. Die deskriptive Statistik zeigt, dass der risikofreudigere Investor (RRA 4) einen stärkeren Anteil in der Rohstoffklasse als der risikoaversere Investor (RRA 8) hält. Dies ist im Einklang mit den vorherigen Überlegungen, 
dass der risikofreudigere Investor den hohen Excess Return des XAU Index "würdigt" bzw. der risikoaversere die höhere Volatilität "meidet".

Um den höheren Anteil im XAU Index auszugleichen, verringert der risikofreudigere Investor die Positionen fast ausschließlich im TNX Index. Die Reduktion der langfristigen Anleihe im Portfolio ist über alle Strategien insbesondere am Median und an den entsprechenden p-Werten zu erkennen. Zusammenfassend ist die Two-Fund Separation für das betrachtete Szenario nicht haltbar.

\subsubsection{Berücksichtigung von Transaktionskosten}

Implizite Strategien zeichnen sich gegenüber historischen Benchmark Strategien in vielen Fällen durch einen erhöhten Turnover aus. ${ }^{31}$ Dies dürfte darauf zurückzuführen sein, dass implizite Strategien unmittelbar auf veränderte Marktsituationen reagieren können und diese "neuen" Informationen in die optimale Asset Allokation einfließen lassen. Historische Verfahren reagieren hingegen verzögert auf diese Informationen, da sie aktuelle Ereignisse nur gemittelt im Nutzenkalkül berücksichtigen. Dass die bessere out-of-sample Performance der impliziten Strategien gegenüber den historischen Benchmark Strategien im Falle von Transaktionskosten weiterhin gilt, zeigt Tabelle 3.13.

31 Vgl. Kostakis, Panigirtzoglou und Skiadopoulos (2011), DeMiguel, Plyakha, Uppal und Vilkov (2012) und Kempf, Korn und Saßning (2012). 
Tabelle 3.13: Turnover sowie um Transaktionskosten bereinigte Sicherheitsäquivalente und Excess Returns der aktiven Strategien

\begin{tabular}{|c|c|c|c|c|c|c|}
\hline & $\widehat{C E Q}-\mathrm{TC}$ & $\hat{\mu}-\mathrm{TC}$ & TO & $\widehat{C E Q}-\mathrm{TC}$ & $\hat{\mu}-\mathrm{TC}$ & TO \\
\hline $\mathrm{RRA}=2$ & & $\mathrm{~N}=3$ & & & $\mathrm{~N}=4$ & \\
\hline $1 / \mathrm{N}$ & $-0,70 \%$ & $-0,30 \%$ & $2,15 \%$ & $1,74 \%$ & $2,94 \%$ & $3,62 \%$ \\
\hline $36 \mathrm{M}$ & $-2,20 \%$ & $-1,57 \%$ & $20,08 \%$ & $-1,51 \%$ & $1,25 \%$ & $20,96 \%$ \\
\hline $60 \mathrm{M}$ & $-3,71 \%$ & $-2,96 \%$ & $19,11 \%$ & $-1,28 \%$ & $0,91 \%$ & $21,53 \%$ \\
\hline $120 \mathrm{M}$ & $-3,02 \%$ & $-2,43 \%$ & $9,33 \%$ & $-0,76 \%$ & $1,49 \%$ & $13,90 \%$ \\
\hline Implizit & $-1,40 \%$ & $-1,03 \%$ & $27,73 \%$ & $-0,01 \%$ & $1,83 \%$ & $26,40 \%$ \\
\hline \multicolumn{7}{|l|}{$\mathrm{RRA}=4$} \\
\hline $1 / \mathrm{N}$ & $-1,10 \%$ & $-0,30 \%$ & $2,15 \%$ & $0,52 \%$ & $2,94 \%$ & $3,62 \%$ \\
\hline $36 \mathrm{M}$ & $-3,08 \%$ & $-1,98 \%$ & $17,77 \%$ & $-2,43 \%$ & $2,84 \%$ & $21,16 \%$ \\
\hline $60 \mathrm{M}$ & $-3,79 \%$ & $-2,65 \%$ & $16,60 \%$ & $-3,19 \%$ & $0,48 \%$ & $20,24 \%$ \\
\hline $120 \mathrm{M}$ & $-2,99 \%$ & $-1,80 \%$ & $8,13 \%$ & $-2,00 \%$ & $1,51 \%$ & $12,16 \%$ \\
\hline Implizit & $-1,78 \%$ & $-1,16 \%$ & $24,75 \%$ & $-1,59 \%$ & $0,31 \%$ & $26,53 \%$ \\
\hline \multicolumn{7}{|l|}{$\mathrm{RRA}=6$} \\
\hline $1 / \mathrm{N}$ & $-1,50 \%$ & $-0,30 \%$ & $2,15 \%$ & $-0,74 \%$ & $2,94 \%$ & $3,62 \%$ \\
\hline $36 \mathrm{M}$ & $-4,33 \%$ & $-2,54 \%$ & $17,70 \%$ & $-5,42 \%$ & $1,21 \%$ & $21,40 \%$ \\
\hline $60 \mathrm{M}$ & $-3,79 \%$ & $-1,95 \%$ & $16,17 \%$ & $-4,57 \%$ & $-0,02 \%$ & $19,58 \%$ \\
\hline $120 \mathrm{M}$ & $-3,42 \%$ & $-1,70 \%$ & $7,51 \%$ & $-3,35 \%$ & $0,70 \%$ & $12,05 \%$ \\
\hline Implizit & $-1,77 \%$ & $-0,93 \%$ & $22,63 \%$ & $-2,17 \%$ & $-0,36 \%$ & $25,96 \%$ \\
\hline \multicolumn{7}{|l|}{$\mathrm{RRA}=8$} \\
\hline $1 / \mathrm{N}$ & $-1,90 \%$ & $-0,30 \%$ & $2,15 \%$ & $-2,04 \%$ & $2,94 \%$ & $3,62 \%$ \\
\hline $36 \mathrm{M}$ & $-4,18 \%$ & $-1,89 \%$ & $17,75 \%$ & $-6,86 \%$ & $0,81 \%$ & $20,30 \%$ \\
\hline $60 \mathrm{M}$ & $-4,29 \%$ & $-2,16 \%$ & $14,81 \%$ & $-5,23 \%$ & $-0,36 \%$ & $19,50 \%$ \\
\hline $120 \mathrm{M}$ & $-3,66 \%$ & $-1,72 \%$ & $7,72 \%$ & $-3,73 \%$ & $0,51 \%$ & $10,85 \%$ \\
\hline Implizit & $-1,87 \%$ & $-0,94 \%$ & $21,10 \%$ & $-2,30 \%$ & $-0,64 \%$ & $26,01 \%$ \\
\hline
\end{tabular}

Diese Tabelle zeigt die um Transaktionskosten bereinigten Sicherheitsäquivalente und Excess Returns sowie den Turnover der aktiven Strategien für Investoren mit CRRA Nutzenfunktionen. 1/N bezeichnet die entsprechende passive Strategie. Die Sicherheitsäquivalente und Excess Returns sind annualisiert. Der out-of-sample Zeitraum beträgt Februar 1999 bis Oktober 2010.

Es lässt sich die Beobachtung bestätigen, dass implizite Strategien einen höheren Turnover haben. Die Sicherheitsäquivalente zeigen unter Berücksichtigung von Transaktionskosten weiterhin bessere Ergebnisse für die impliziten Strategien. Die Ergebnisse und Resultate aus Tabelle 3.7 werden unter Berücksichtigung von Transaktionskosten somit bestätigt.

Weiter ist zu beobachten, dass für steigende Risikoaversionen für alle betrachteten aktiven Strategien der Turnover sinkt. Dies lässt sich folgendermaßen begründen. Je risikoaverser der Investor ist, desto weniger werden extreme Positionen in den Assets eingegangen und somit wird in den Beobachtungszeitpunkten nach Bildung neuer Erwartungen das Portfolio weniger stark umgeschichtet. 


\subsection{Diskussion}

In diesem Kapitel wurde die strategische Asset Allokation für Investoren mit CRRA Nutzenfunktionen anhand von zwei Anlageuniversen untersucht. Im Rahmen eines traditionellen Anlageuniversums $(\mathrm{N}=3)$ hatten die Investoren die Möglichkeit, ihr Vermögen in eine risikofreie Anlage sowie in einen Aktienindex und eine langfristige Anleihe zu investieren. Für das zweite Anlageuniversum $(\mathrm{N}=4)$ wurde das traditionelle Universum um die Investitionsmöglichkeit in eine Rohstoffklasse ergänzt.

Die Nutzenvorteile durch die Hinzunahme von Rohstoffen sind in der Literatur strittig ${ }^{32}$ und wurden im Rahmen der empirischen Studie reflektiert. Die Ergebnisse zeigen für risikofreudigere Investoren (RRA 2 und 4) einen positiven Beitrag, für risikoaversere Investoren (RRA 6 und 8) jedoch nicht. Eine allgemeine Handelsempfehlung lässt sich somit nicht ableiten und liefert ein differenziertes Resultat in Abhängigkeit der Risikoaffinität des Investors.

Zur Untersuchung des Einflusses der höheren Momente Schiefe und Kurtosis wurde die optimale Asset Allokation für Investoren mit unterschiedlichen Risikoaversionen analysiert und verglichen. Da keine normalverteilten Renditen unterstellt und höhere Momente im Kalkül berücksichtigt werden, kommt die Risikoaversion nicht nur in der Aufteilung zwischen riskanter und risikofreier Anlage zum Ausdruck, sondern auch in der Komposition des riskanten Anteils im Portfolio. Es wurde allerdings empirisch festgestellt, dass für das Anlageuniversum $\mathrm{N}=3$ die Asset Allokation im Wesentlichen durch eine Two-Fund Separation beschrieben werden kann. Im erweiterten Universum variiert die Komposition des riskanten Portfolioanteils jedoch deutlich.

Über alle betrachteten Strategien wurde festgestellt, dass der risikofreudigere gegenüber dem risikoaverseren Investor einen größeren Anteil an Rohstoffen im Gegenzug für den Verzicht von langfristigen Anleihen hält. Dieses Resultat hat weitreichende Implikationen für die Praxis. Es zeigt, dass sich die Risikoaffinität des Investors in einem Anlageuniversum mit einer Rohstoffklasse nicht nur im Gewicht der risikofreien Anlage, sondern auch in der Komposition des riskanten Anteils des Portfolios widerspiegeln sollte.

Zur Beurteilung, ob implizite Informationen im Rahmen der strategischen Asset Allokation vorteilhaft sind, wurde die Performance der impliziten Strategie passiven und aktiven Benchmarks gegenübergestellt. Die Ergebnisse zeigen, dass aktive Strategien, die auf impliziten Informationen basieren, historischen Benchmark Strategien in Bezug auf das Sicherheitsäquivalent und auf die realisierte Volatilität vorzuziehen sind. Es wurde ver-

32 Vgl. Daskalaki und Skiadopoulos (2011). 
deutlicht, dass die Out-Performance auf eine verbesserte Schätzung des Erwartungswertes zurückzuführen ist. Hieraus resultiert eine stärkere Gewichtung der risikofreien Anlage und somit eine signifikant geringere realisierte Volatilität. Diese Resultate sind bei Hinzunahme von Transaktionskosten weiterhin robust. Im Vergleich zu passiven Strategien und im Besonderen zu der 1/N Strategie konnte die implizite Strategie jedoch keine überzeugende Performance liefern. ${ }^{33}$ Insgesamt lässt sich festhalten, dass die implizite Strategie gegenüber aktiven Strategien auf Basis historischer Renditezeitreihen Nutzenvorteile erzielt, gegenüber einer $1 / \mathrm{N}$ Strategie jedoch nicht.

In diesem Kapitel wurden die zentralen Forschungsfragen dieser Arbeit im Rahmen der strategischen Asset Allokation untersucht. Die verwendete Methodik lässt sich allerdings nicht auf den Fall der taktischen Asset Allokation übertragen. Für (deutlich) mehr riskante Assets ist insbesondere die Integration einer beliebigen multidimensionalen Dichte numerisch nicht leistbar. Falls dennoch der modellfreie Ansatz zur Extraktion der impliziten Informationen weiter verfolgt werden soll, müssen modellfreie implizite Momente nach Britten-Jones und Neuberger (2000) oder Bakshi, Kapadia und Madan (2003) betrachtet werden. Diese Momente werden wiederum unter dem risikoneutralen Maß gebildet und sind somit nicht in das physische Maß transformiert bzw. um Risikoprämien bereinigt. Um dieser Problematik zu begegnen, wird im folgenden Kapitel das Konzept der risikoadjustierten modellfreien impliziten Momente eingeführt. Die taktische Asset Allokation mit impliziten Information erfolgt im darauffolgenden Kapitel.

33 Die Ergebnisse sind im Einklang mit der bestehenden Literatur (vgl. Kostakis, Panigirtzoglou und Skiadopoulos (2011), DeMiguel, Garlappi und Uppal (2009b) und Fugazza, Guidolin und Nicodano (2010)). 



\section{Kapitel 4}

\section{Risikoadjustierte modellfreie implizite Momente}

\section{$4.1 \quad$ Einführung}

Zur Untersuchung der Asset Allokation mit impliziten Informationen wurde im vorherigen Kapitel die modellfreie implizite Dichte nach Breeden und Litzenberger (1978) herangezogen. Die Verwendung einer vollständigen Dichte ist für den Fall vieler riskanter Anlagewerte jedoch problematisch, da zur Bestimmung der Momente jeweils über die vollständige Dichte integriert werden muss. Daher hat sich die Verwendung direkt abgeleiteter risikoneutraler Momente aus dem modellfreien Ansatz von Bakshi, Kapadia und Madan (2003) auch in der Asset Allokation etabliert. ${ }^{1}$ Diese basieren auf einem Kontinuum von Out-of-the-Money Put und Call Optionen und können ohne Verwendung numerischer Ableitungen bestimmt werden. ${ }^{2}$ Dies ist von großem Vorteil, denn numerische Ableitungen bergen einerseits große Ungenauigkeiten bei der Ermittlung der Momente ${ }^{3}$ und können andererseits nicht sicherstellen, dass die resultierende Wahrscheinlichkeitsdichte nur nicht negative Werte besitzt ${ }^{4}$.

Allerdings werden die impliziten Momente unter dem risikoneutralen Wahrscheinlichkeitsmaß gebildet und haben somit aus theoretischer Sicht nur bedingte Aussagefähigkeit für die Verteilung der Anlagewerte unter dem physischen Maß. Um implizite Momente unter

1 Die modellfreien impliziten Momente von Bakshi, Kapadia und Madan (2003) kommen in einer Vielzahl von wissenschaftlichen Beiträgen zur Anwendung. Siehe hierzu eine Übersicht in Chang, Christoffersen und Jacobs (2011b).

2 In diesem Kapitel werden ausschließlich Optionen europäischen Typs betrachtet.

3 Vgl. z. B. Jondeau und Rockinger (2001).

4 Vgl. z. B. Abbildung 3.3. 
dem physischen Maß zu erhalten, muss eine Risikoadjustierung vorgenommen werden. Dass die Risikoadjustierung nicht zu vernachlässigen ist, kann z. B. empirisch anhand der Varianz-Risikoprämie beobachtet werden.

Um das Problem der Überführung vom risikoneutralen zum physischen Maß für die Momente direkt zu leisten, werden in diesem Kapitel risikoadjustierte modellfreie implizite Momente hergeleitet. Die Transformation basiert dabei ausschließlich auf der Nutzenfunktion eines repräsentativen Investors sowie auf Out-of-the-Money Optionen. Die hergeleiteten impliziten Momente stellen eine direkte Erweiterung der Momente von Bakshi, Kapadia und Madan (2003) dar und sind für den Spezialfall, dass der repräsentative Investor risikoneutral ist, in das erarbeitete Konzept eingebettet. Bevor jedoch die hergeleiteten impliziten Momente in der taktischen Asset Allokation zur Anwendung kommen, werden zunächst Wirkungen der Risikoadjustierung auf die Momente sowie die Prognosegüte der realisierten Momente für den S\&P 500 untersucht.

Empirische Studien, welche insbesondere die Wirkung des Überganges vom risikoneutralen Maß zum physischen untersuchen und eine vergleichbare Methodik verwenden, sind die Beiträge von Duan und Zhang (2010) und Karoui (2011). Zentrale Größe dieser Studien ist die aus einem Vergleich von impliziten und physischen Momenten eines Indexes gewonnene Markt-Risikoprämie (Excess Return).

Duan und Zhang (2010) benutzen zur Transformation der risikoneutralen Dichte einen repräsentativen Investor mit einer exponentiellen Nutzenfunktion gemäß Bakshi, Kapadia und Madan (2003). ${ }^{5}$ Mit Hilfe der charakteristischen Funktion der physischen Verteilung wird der Drift des riskanten Assets approximativ durch die physischen höheren Momente bis zur vierten Ordnung und die Risikoaversion des repräsentativen Investors ausgedrückt. Letztere schätzen Duan und Zhang (2010) mit Hilfe des Volatility-Spreads ${ }^{6}$ gemäß Bakshi und Madan (2006). Die durchschnittlich geschätzte Risikoaversion für den S\&P 500 ist 4,25. Dieses Resultat steht im Einklang mit der geschätzten Risikoaversion von 4,08 in Bliss und Panigirtzoglou (2004).

Karoui (2011) konstruiert mit Hilfe von No-Arbitrage Argumenten Portfolios, die den Drift eines riskanten Assets duplizieren. Hierzu wird die Annahme getroffen, dass neben der risikofreien Anlage und dem riskanten Asset weitere Instrumente zu Duplikationszwecken bzw. zur Vervollständigung des Marktes zur Verfügung stehen. Dies ermöglicht es, auf die Existenz eines repräsentativen Investors zu verzichten. Für die Anwendung werden die von Bakshi, Kapadia und Madan (2003) eingeführten Volatility-, Cubic- und Quartic-

$5 \quad$ Die Verwendung einer CARA Nutzenfunktion zur Transformation der Renditen entspricht in diesem Kontext einer CRRA Nutzenfunktion zur Transformation des Kurses.

6 Der Volatility-Spread entspricht der relativen Varianz-Risikoprämie. 
Kontrakte betrachtet. Die untersuchte Markt-Risikoprämie wird schließlich aus einer gewichteten Differenz zwischen dem Kontrakt und dem entsprechenden physischen Moment gewonnen. Die auf diese Weise bestimmte Markt-Risikoprämie outperformt klassische historische Verfahren, wie in Goyal und Welch (2008), und liefert ein Bestimmtheitsmaß für die Prognose von realisierten Excess Returns von $R^{2}=10 \%$.

Die Progonosegüte impliziter Momente für entsprechende realisierte Momente wurde insbesondere für die Volatilität untersucht. So wurde gezeigt ${ }^{7}$, dass die modellfreie implizite Volatilität, bestimmt durch die Methodik von Bakshi, Kapadia und Madan (2003) oder Britten-Jones und Neuberger (2000), ${ }^{8}$ eine sehr gute Prognosefähigkeit für die realisierte Volatilität liefert. Inwieweit nun der risikoadjustierte Moment, trotz der theoretischen Fundierung, eine Verbesserung der Prognosefähigkeit gegenüber dem rein impliziten darstellt, ist unklar und wird im Folgenden untersucht. Des Weiteren wird auch die Prognosefähigkeit für die Schiefe und Kurtosis, welche in der Literatur noch nicht eingehend betrachtet wurde, getestet. Lediglich untersuchen Kozhan, Neuberger und Schneider (2013) in diesem Zusammenhang den "Skew Swap", wessen Auszahlung der realisierten Schiefe entspricht, für die Prognosefähigkeit der Schiefe.

Der sich anschließende Teil des Kapitels ist wie folgt organisiert. Der nachfolgende Abschnitt leitet das Konzept der risikoadjustierten modellfreien impliziten Momente her. Es folgt die Analyse der Wirkung der Risikoadjustierung auf die impliziten Momente. Weiterführend wird die Fähigkeit zur Prognose realisierter höherer Momente thematisiert. Das Kapitel schließt mit einem Fazit und diskutiert insbesondere Implikationen für die Anwendung in der Asset Allokation.

\subsection{Herleitung risikoadjustierter modellfreier implizi- ter Momente}

Zur Bestimmung risikoadjustierter modellfreier impliziter Momente werden zunächst einige Vorüberlegungen benötigt. Das endgültige Resultat findet sich in Korrolar 2. Hierzu werden in einem ersten Schritt in Lemma 1 die erwarteten diskontierten Auszahlungen von Call- und Put-Optionen unter dem physischen Maß betrachtet. Das Ergebnis stellt

$7 \quad$ Vgl. Literaturüberblick in der Einleitung.

8 Die modellfreie implizite Volatilität nach Britten-Jones und Neuberger (2000) stimmt nicht zwangsweise mit der entsprechenden Volatiltät von Bakshi, Kapadia und Madan (2003) überein. Der wesentliche Unterschied besteht darin, dass bei dieser Methodik nicht der Moment der Log-Rendite bestimmt wird, sondern die erwartete quadratische Variation des zugrundeliegenden stochastischen Prozesses. Eine Erweiterung der Methodik von Britten-Jones und Neuberger (2000) zur Berücksichtung von Jumps wurde in Jiang und Tian (2005) entwickelt. 
den wesentlichen theoretischen Beitrag des Kapitels dar. Darauf aufbauend werden in Analogie zu Bakshi, Kapadia und Madan (2003) in einem zweiten Schritt die diskontierten nicht-zentralen Momente der Log-Rendite Verteilung unter dem physischen Maß betrachtet. Das Ergebnis präsentiert Satz 1. Korrolar 1 liefert die für Anwendungen wichtigen Werte des Volatility-, Cubic- und Quartic-Kontraktes. Finalisierend wird in Satz 2 hergeleitet, wie die Markt-Risikoprämie bzw. der Erwartungswert der Log-Rendite unter dem physischen Maß approximativ bestimmt werden kann. Korrolar 2 liefert schließlich die Varianz, Schiefe und Kurtosis der Log-Rendite Verteilung.

Lemma 1: Es existiere ein repräsentativer Investor mit einer Nutzenfunktion aus der Klasse $^{9}$

$$
U \in \mathcal{A}_{\infty}=\left\{U:(-1)^{k} U^{(k+1)}(x) \geq 0 \text { für alle } x>0 \text { und } k=0,1,2, \ldots\right\} .
$$

Für ein Asset $S_{t}$, mit $S_{t}>0$ Q-f.s., und dem deterministischen Zinssatz $r>0$ sei die erwartete diskontierte Auszahlung unter dem physischen Maß (kurz: EDAP) zum Zeitpunkt $t$ für den Call und Put mit Laufzeit $\tau$ und Ausübungspreis $K$ definiert durch

$$
\begin{aligned}
& C^{P}(t, \tau, K):=E^{P}\left\{e^{-r \tau}\left(S_{t+\tau}-K\right)^{+}\right\}, \\
& P^{P}(t, \tau, K):=E^{P}\left\{e^{-r \tau}\left(K-S_{t+\tau}\right)^{+}\right\} .
\end{aligned}
$$

Die EDAP's für den Call (4.1) und für den Put (4.2) können durch die Marktpreise ${ }^{10}$ von Call-, Put- und Digitaloptionen, bezeichnet mit $C(t, \tau, K), P(t, \tau, K)$ und $D(t, \tau, K)$, sowie der Nutzenfunktion des repräsentativen Investors bestimmt werden.

Der Call $C^{P}(t, \tau, K)$ durch

$$
\frac{C(t, \tau, K)}{c \cdot U^{\prime}(K)}+\int_{K}^{\infty} \frac{-U^{\prime \prime}(x)}{c \cdot U^{\prime}(x)^{2}}\{C(t, \tau, x)+(x-K) D(t, \tau, x)\} d x
$$

$9 \quad \mathcal{A}_{\infty}$ entspricht der Klasse von Nutzenfunktionen, deren Grenznutzen mittels des Lemmas von Bernstein ausgedrückt werden kann durch

$$
U^{\prime}(x)=\int \exp (-r x) \mu(d r), \quad x>0,
$$

wobei $r$ den stetigen risikolosen Zinssatz und $\mu$ ein spezifisches Maßs bezeichnet. Funktionen $U \in \mathcal{A}_{\infty}$ haben die Eigenschaft der vollständigen Monotonie. Für eine ausführliche Diskussion dieser Klasse von Nutzenfunktionen, welche unter anderem die HARA-Klasse enthält, siehe z. B. Brockett und Golden (1987) und Caballé und Pomansky (1996).

10 Die Marktpreise entsprechen den erwarteten diskontierten Auszahlungen unter dem risikoneutralen Wahrscheinlichkeitsmaß. 
der Put $P^{P}(t, \tau, K)$ durch

$$
\frac{P(t, \tau, K)}{c \cdot U^{\prime}(K)}-\int_{0}^{K} \frac{-U^{\prime \prime}(x)}{c \cdot U^{\prime}(x)^{2}}\left\{P(t, \tau, x)+(K-x)\left(e^{-r \tau}-D(t, \tau, x)\right)\right\} d x
$$

wobei die Normierungskonstante $c$ bestimmt wird durch

$$
c=\int_{0}^{\infty} \frac{-U^{\prime \prime}(x)}{U^{\prime}(x)^{2}} e^{r \tau} D(t, \tau, x) d x+\frac{1}{U^{\prime}(0)} .
$$

Beweis: Der Beweis verwendet einen Satz aus der Maßtheorie. ${ }^{11}$

Sei $(\Omega, \mathcal{A}, \mu)$ ein endlicher Maßraum, $f$ eine nichtnegative reelle Funktion und $\varphi:[0, \infty) \rightarrow$ $[0, \infty)$ monoton steigend, stetig differenziebar mit $\varphi(0)=0$, dann gilt

$$
\int \varphi \circ f d \mu=\int_{0}^{\infty} \varphi^{\prime}(t) \mu(f>t) d t
$$

Da $U \in \mathcal{A}_{\infty}$, hat die Funktion $\frac{1}{U^{\prime}(x)}$ folgende Eigenschaften:

(i) Als Verknüpfung stetig differenzierbarer Funktionen ist $\frac{1}{U^{\prime}(x)}$ stetig differenzierbar.

(ii) Da $\left(\frac{1}{U^{\prime}(x)}\right)^{\prime}=\frac{-U^{\prime \prime}(x)}{U^{\prime}(x)^{2}}>0$ für alle $x>0$, ist $\frac{1}{U^{\prime}(x)}$ monoton steigend.

(iii) Für $x \rightarrow 0$ ist $\frac{1}{U^{\prime}(x)}$ monoton fallend und beschränkt gegen 0 und konvergiert somit gegen $\frac{1}{U^{\prime}(0)} \geq 0$.

Zusammen erfüllt $\frac{1}{U^{\prime}(x)}-\frac{1}{U^{\prime}(0)}$ die Bedingungen an $\varphi$ aus dem obigen Satz.

Die Dichte des physischen Maßes $p$ wird gemäß (3.1) gegeben durch

$$
p\left(S_{t}\right)=\frac{q\left(S_{t}\right)}{c \cdot U^{\prime}\left(S_{t}\right)}, \quad \text { mit } \quad c:=\int \frac{q(x)}{U^{\prime}(x)} d x .
$$

Für den Call muss nach Definition (4.1) folgendes Integral betrachtet werden

$$
\begin{aligned}
& E^{P}\left\{e^{-r \tau}\left(S_{t+\tau}-K\right)^{+}\right\} \\
= & \int_{0}^{\infty} e^{-r \tau}\left(S_{t+\tau}-K\right)^{+} P\left(d S_{t+\tau}\right)
\end{aligned}
$$

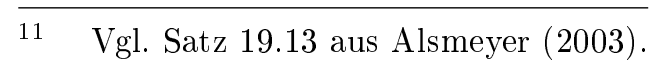




$$
\begin{aligned}
& =\frac{1}{c} \cdot \int_{0}^{\infty} e^{-r \tau}\left(S_{t+\tau}-K\right)^{+} \frac{1}{U^{\prime}\left(S_{t+\tau}\right)} Q\left(d S_{t+\tau}\right) \\
& =\frac{1}{c} \cdot \int_{0}^{\infty}\left\{\frac{1}{U^{\prime}\left(S_{t+\tau}\right)}-\frac{1}{U^{\prime}(0)}+\frac{1}{U^{\prime}(0)}\right\} \underbrace{e^{-r \tau}\left(S_{t+\tau}-K\right)^{+} Q\left(d S_{t+\tau}\right)}_{=: \tilde{P_{C}}\left(d S_{t+\tau}\right)} \\
& =\frac{1}{c} \cdot \int_{0}^{\infty} \frac{-U^{\prime \prime}(x)}{U^{\prime}(x)^{2}} \tilde{P}_{C}\left\{S_{t+\tau}>x\right\} d x+\frac{C(t, \tau, K)}{c \cdot U^{\prime}(0)} \\
& =\frac{1}{c} \cdot \int_{0}^{\infty} \frac{-U^{\prime \prime}(x)}{U^{\prime}(x)^{2}}\left\{\int_{x}^{\infty} e^{-r \tau}\left(S_{t+\tau}-K\right)^{+} Q\left(d S_{t+\tau}\right)\right\} d x+\frac{C(t, \tau, K)}{c \cdot U^{\prime}(0)},
\end{aligned}
$$

wobei in der vorletzten Zeile der obige Satz benutzt wurde.

Das innere Integral $\int_{x}^{\infty} e^{-r \tau}\left(S_{t+\tau}-K\right)^{+} Q\left(d S_{t+\tau}\right)$ entspricht für $x<K$ dem Wert einer Call-Option zum Ausübungspreis $K$. Für $x>K$ hingegen ergibt sich

$$
\begin{aligned}
& \int_{x}^{\infty} e^{-r \tau} \underbrace{\left(S_{t+\tau}-K\right)^{+}}_{>0} Q\left(d S_{t+\tau}\right) \\
= & \int_{x}^{\infty} e^{-r \tau}\left(S_{t+\tau}-x\right) Q\left(d S_{t+\tau}\right)+\int_{x}^{\infty} e^{-r \tau}(x-K) Q\left(d S_{t+\tau}\right) \\
= & C(t, \tau, x)+(x-K) \underbrace{e^{-r \tau} Q\left\{S_{t+\tau}>x\right\}}_{\text {Digital-Option zum Ausübungspreis x }} \\
= & C(t, \tau, x)+(x-K) D(t, \tau, x) .
\end{aligned}
$$

Eingesetzt folgt für den Call die Behauptung

$$
\begin{aligned}
& C^{P}(t, \tau, K) \\
= & \frac{1}{c} \cdot \int_{0}^{\infty} \frac{-U^{\prime \prime}(x)}{U^{\prime}(x)^{2}} C(t, \tau, K) \mathbb{1}_{\{x<K\}} d x+\frac{C(t, \tau, K)}{c \cdot U^{\prime}(0)} \\
& +\frac{1}{c} \cdot \int_{0}^{\infty} \frac{-U^{\prime \prime}(x)}{U^{\prime}(x)^{2}}\{C(t, \tau, x)+(x-K) D(t, \tau, x)\} \mathbb{1}_{\{x>K\}} d x \\
= & \frac{1}{c} \cdot C(t, \tau, K)\left\{\frac{1}{U^{\prime}(K)}-\frac{1}{U^{\prime}(0)}\right\}+\frac{C(t, \tau, K)}{c \cdot U^{\prime}(0)} \\
& +\frac{1}{c} \cdot \int_{K}^{\infty} \frac{-U^{\prime \prime}(x)}{U^{\prime}(x)^{2}}\{C(t, \tau, x)+(x-K) D(t, \tau, x)\} d x .
\end{aligned}
$$


Mit einer analogen Argumentation folgt für den Put (vgl. Definition (4.2)):

$$
\begin{aligned}
& E^{P}\left\{e^{-r \tau}\left(K-S_{t+\tau}\right)^{+}\right\} \\
= & \int_{0}^{\infty} e^{-r \tau}\left(K-S_{t+\tau}\right)^{+} P\left(d S_{t+\tau}\right) \\
= & \frac{1}{c} \cdot \int_{0}^{\infty} e^{-r \tau}\left(K-S_{t+\tau}\right)^{+} \frac{1}{U^{\prime}\left(S_{t+\tau}\right)} Q\left(d S_{t+\tau}\right) \\
= & \frac{1}{c} \cdot \int_{0}^{\infty}\left\{\frac{1}{U^{\prime}\left(S_{t+\tau}\right)}-\frac{1}{U^{\prime}(0)}+\frac{1}{U^{\prime}(0)}\right\} \underbrace{e^{-r \tau}\left(K-S_{t+\tau}\right)^{+} Q\left(d S_{t+\tau}\right)}_{=: \tilde{P_{P}\left(d S_{t+\tau}\right)}} \\
= & \frac{1}{c} \cdot \int_{0}^{\infty} \frac{-U^{\prime \prime}(x)}{U^{\prime}(x)^{2}} \tilde{P}_{P}\left\{S_{t+\tau}>x\right\} d x+\frac{P(t, \tau, K)}{c \cdot U^{\prime}(0)} \\
= & \frac{1}{c} \cdot \int_{0}^{\infty} \frac{-U^{\prime \prime}(x)}{U^{\prime}(x)^{2}}\left\{\int_{x}^{\infty} e^{-r \tau}\left(K-S_{t+\tau}\right)^{+} Q\left(d S_{t+\tau}\right)\right\} d x+\frac{P(t, \tau, K)}{c \cdot U^{\prime}(0)} .
\end{aligned}
$$

Für $x>K$ ist $\int_{x}^{\infty} e^{-r \tau}\left(K-S_{t+\tau}\right)^{+} Q\left(d S_{t+\tau}\right)=0$. Falls $x<K$, gilt

$$
\begin{aligned}
& \int_{x}^{\infty} e^{-r \tau}\left(K-S_{t+\tau}\right)+Q\left(d S_{t+\tau}\right) \\
= & \int_{0}^{K} e^{-r \tau}\left(K-S_{t+\tau}\right) Q\left(d S_{t+\tau}\right)-\int_{0}^{x} e^{-r \tau}\left(K-S_{t+\tau}\right) Q\left(d S_{t+\tau}\right) \\
= & P(t, \tau, K)-\int_{0}^{x} e^{-r \tau}\left(x-S_{t+\tau}\right) Q\left(d S_{t+\tau}\right)-(K-x) e^{-r \tau} \underbrace{\int_{0}^{x} Q\left(d S_{t+\tau}\right)}_{=1-Q\left\{S_{t+\tau}>x\right\}} \\
= & P(t, \tau, K)-P(t, \tau, x)-(K-x)\left(e^{-r \tau}-D(t, \tau, x)\right) .
\end{aligned}
$$

Eingesetzt folgt für den Put die Behauptung

$$
\begin{aligned}
& P^{P}(t, \tau, K) \\
= & \frac{1}{c} \cdot \int_{0}^{\infty} \frac{-U^{\prime \prime}(x)}{U^{\prime}(x)^{2}} P(t, \tau, K) \mathbb{1}_{\{x<K\}} d x+\frac{P(t, \tau, K)}{c \cdot U^{\prime}(0)} \\
& -\frac{1}{c} \cdot \int_{0}^{\infty}\left\{P(t, \tau, x)+(K-x)\left(e^{-r \tau}-D(t, \tau, x)\right)\right\} \mathbb{1}_{\{x<K\}} d x
\end{aligned}
$$




$$
\begin{aligned}
= & \frac{1}{c} \cdot P(t, \tau, K)\left\{\frac{1}{U^{\prime}(K)}-\frac{1}{U^{\prime}(0)}\right\}+\frac{P(t, \tau, K)}{c \cdot U^{\prime}(0)} \\
& -\frac{1}{c} \cdot \int_{0}^{K} \frac{-U^{\prime \prime}(x)}{U^{\prime}(x)^{2}}\left\{P(t, \tau, x)+(K-x)\left(e^{-r \tau}-D(t, \tau, x)\right)\right\} d x .
\end{aligned}
$$

Die Normierungskonstante $c$ lässt sich mit analogen Argumenten bestimmen:

$$
\begin{aligned}
& \int_{0}^{\infty}\left\{\frac{1}{U^{\prime}\left(S_{t+\tau}\right)}-\frac{1}{U^{\prime}(0)}+\frac{1}{U^{\prime}(0)}\right\} Q\left(d S_{t+\tau}\right) \\
= & \int_{0}^{\infty} \frac{-U^{\prime \prime}(x)}{U^{\prime}(x)^{2}}\left\{\int_{x}^{\infty} Q\left(d S_{t+\tau}\right)\right\} d x+\frac{1}{U^{\prime}(0)} \\
= & \int_{0}^{\infty} \frac{-U^{\prime \prime}(x)}{U^{\prime}(x)^{2}} e^{r \tau} D(t, \tau, x) d x+\frac{1}{U^{\prime}(0)} .
\end{aligned}
$$

Für einen risikoneutralen repräsentativen Investor gilt, dass die physische Dichte mit der risikoneutralen übereinstimmt und somit die EDAP's gleich den entsprechenden Marktpreisen sind. Dies lässt sich leicht aus dem obigen Satz erkennen, denn für einen risikoneutralen repräsentativen Investor ist die erste Ableitung seiner Nutzenfunktion konstant eins, die zweite Ableitung folglich null.

$$
U^{\prime}(\cdot) \equiv 1 \quad \Rightarrow \quad U^{\prime \prime}(\cdot) \equiv 0
$$

Damit gilt, dass die Normierungskonstante $c$ gleich eins ist und somit die EDAP's mit den Marktpreisen $C(t, \tau, K)$ und $P(t, \tau, K)$ übereinstimmen. Tabelle 4.1 zeigt zusammenfassend für klassische Nutzenfunktionen die benötigten Terme für die Risikoadjustierung.

Tabelle 4.1: Parameter für die Risikoadjustierung bei konstanter absoluter Risikoaversion (CARA) und konstanter relativer Risikoaversion (CRRA)

\begin{tabular}{ccccc}
\hline & $U\left(S_{t}\right)$ & $\frac{1}{U^{\prime}\left(S_{t}\right)}$ & $\frac{-U^{\prime \prime}\left(S_{t}\right)}{U^{\prime}\left(S_{t}\right)^{2}}$ & RRA \\
\hline CARA & $-\frac{e^{-\gamma S_{t}}}{\gamma}$ & $e^{\gamma S_{t}}$ & $\gamma e^{\gamma S_{t}}$ & $\gamma S_{t}$ \\
CRRA & $\frac{S_{t}^{1-\gamma}-1}{1-\gamma}$ & $S_{t}^{\gamma}$ & $\gamma S_{t}^{\gamma-1}$ & $\gamma$ \\
\hline
\end{tabular}

Diese Tabelle zeigt für Nutzenfunktionen mit konstanter absoluter Risikoaversion (CARA) und konstanter relativer Risikoaversion (CRRA) die entsprechenden Koeffizienten für die Formel der EDAP's. Für $\gamma>$ 0 gehören diese Nutzenfunktionen zur Klasse $\mathcal{A}_{\infty}$. Mit RRA ist die relative Risikoaversion $\frac{-S_{t} U^{\prime \prime}\left(S_{t}\right)}{U^{\prime}\left(S_{t}\right)}$ bezeichnet. 
Im Folgenden wird ein Beispiel eingeführt, welches zeigt, dass sich im Falle log-normalverteilter Kurse mit einem repräsentativen CRRA Investor die bekannte Black-Scholes Formel für den Call mit Drift $r+\gamma \sigma^{2}$ ergibt. Dies ist im Einklang mit dem im vorherigen Kapitel diskutierten Beispiel, im Rahmen dessen gezeigt wurde, dass die Risikoadjustierung vor allem den Erwartungswert steuert. Das Beispiel zeigt auch, dass im Fall log-normalverteilter Kurse die EDAP's durch geschlossene Formeln dargestellt werden können.

\section{Beispiel der Risikoadjustierung bei log-normalverteilten Kursen}

Der Kurs $S_{t+\tau}$ besitzt unter dem risikoneutralen Maß eine $\mathcal{L} \mathcal{N}\left(r, \sigma^{2}\right)$ Verteilung. Dann gilt, dass der Wert einer Digitaloption gerade der negativen Ableitung des Call-Preises nach dem Ausübungspreis entspricht

$$
D(t, \tau, K)=-\frac{d}{d K} C(t, \tau, K)
$$

Weiter wird angenommen, dass der repräsentative Investor durch eine CRRA Nutzenfunktion mit Risikoaversionsparameter $\gamma \in \mathbb{R}$ (vgl. (2.6) oder Tabelle 4.1) gegeben wird. Nach Lemma 1 wird das EDAP für den Call gegeben durch

$$
\begin{aligned}
C^{P}(t, \tau, K)= & \frac{1}{c} C(t, \tau, K) K^{\gamma}+\frac{1}{c} \underbrace{\int_{K}^{\infty} \gamma x^{\gamma-1} C(t, \tau, x) d x}_{(i)} \\
& +\frac{1}{c} \int_{K}^{\infty} \gamma x^{\gamma-1}(x-K) D(t, \tau, x) d x .
\end{aligned}
$$

Zunächst sei nur das Integral (i) betrachtet. Es folgt mit partieller Integration

$$
\begin{aligned}
\int_{K}^{\infty} \gamma x^{\gamma-1} C(t, \tau, x) d x & \left.=x^{\gamma} C(t, \tau, x)\right]_{K}^{\infty}+\int_{K}^{\infty} x^{\gamma} D(t, \tau, x) d x \\
& =-C(t, \tau, K) K^{\gamma}+\int_{K}^{\infty} x^{\gamma} D(t, \tau, x) d x
\end{aligned}
$$

wobei ausgenutzt wurde, dass $C(t, \tau, x)$ schneller gegen null als $x^{\gamma}$ gegen unendlich strebt. Es verbleibt für $C^{P}(t, \tau, K)$

$$
\frac{1}{c} \int_{K}^{\infty}\left(x^{\gamma}+\gamma x^{\gamma}-K \gamma x^{\gamma-1}\right) D(t, \tau, x) d x .
$$


Durch Anwendung des Satzes von Fubini können die Integrationen vertauscht werden und es folgt

$$
\begin{aligned}
& \frac{1}{c} \int_{K}^{\infty}\left\{\int_{K}^{S_{t+\tau}}(\gamma+1) x^{\gamma}-K \gamma x^{\gamma-1} d x\right\} e^{-r \tau} Q\left(d S_{t+\tau}\right) \\
= & \left.\frac{1}{c} \int_{K}^{\infty}\left\{x^{\gamma+1}-K x^{\gamma}\right]_{K}^{S_{t+\tau}}\right\} e^{-r \tau} Q\left(d S_{t+\tau}\right) \\
= & \frac{1}{c} \int_{K}^{\infty}\left\{S_{t+\tau}^{\gamma+1}-K S_{t+\tau}^{\gamma}-K^{\gamma+1}+K^{\gamma+1}\right\} e^{-r \tau} Q\left(d S_{t+\tau}\right) \\
= & \frac{1}{c} \int_{K}^{\infty} e^{-r \tau}\left(S_{t+\tau}-K\right) S_{t+\tau}^{\gamma} Q\left(d S_{t+\tau}\right) .
\end{aligned}
$$

Da die physische Verteilung $P\left(d S_{t+\tau}\right)$ gerade $\frac{1}{c} S_{t+\tau}^{\gamma} Q\left(d S_{t+\tau}\right)$ entspricht, stimmt dies mit

$$
E^{P}\left\{e^{-r \tau}\left(S_{t+\tau}-K\right)^{+}\right\}
$$

überein. Nach dem Beispiel aus dem vorherigen Kapitel ist $P\left(d S_{t+\tau}\right)$ wiederum lognormalverteilt mit den Parametern $r+\gamma \sigma^{2}$ und $\sigma^{2}$. Dies bedeutet, dass das EDAP für den Call mit Hilfe der Black-Scholes Formel mit Drift $r+\gamma \sigma^{2}$ bestimmt werden kann. ${ }^{12}$

Der folgende Satz zeigt, wie mit Hilfe von Lemma 1 die diskontierten nicht-zentralen Momente der Log-Rendite Verteilung unter dem physischen Maß gewonnen werden können.

Satz 1: Sei $R_{t+\tau}:=\log \left(S_{t+\tau}\right)-\log \left(S_{t}\right)$ die Log-Rendite des Assets $S_{t+\tau}$ und

$$
H_{k}\left(S_{t+\tau}\right)=R_{t+\tau}^{k} \quad \text { für } k=2,3,4, \ldots
$$

12 Je nach Definition und Darstellung der Black-Scholes Formel ist diese entsprechend anzupassen bzw. sind die Parameter adäquat einzusetzen. Beispielhaft sei hier eine Darstellung aus Kapitel 13 in Hull (2006) illustriert. Sei $S \log$-normalverteilt mit Erwartungswert $E\{S\}$ und Varianz $\sigma^{2}$, dann gilt

$$
E\left\{(S-K)^{+}\right\}=E\{S\} \mathcal{N}\left(d_{1}\right)-K \mathcal{N}\left(d_{2}\right),
$$

mit

$$
d_{1}=\frac{\log (E\{S\} / K)+\sigma^{2} / 2}{\sigma}, \quad \text { und } \quad d_{2}=\frac{\log (E\{S\} / K)-\sigma^{2} / 2}{\sigma} .
$$


die entsprechenden Potenzen höherer Ordnung. Dann gilt

$$
E^{P}\left\{e^{-r \tau} H_{k}\left(S_{t+\tau}\right)\right\}=\int_{S_{t}}^{\infty} H_{k}^{\prime \prime}(K) C^{P}(t, \tau, K) d K+\int_{0}^{S_{t}} H_{k}^{\prime \prime}(K) P^{P}(t, \tau, K) d K .
$$

Beweis: Für die Ableitung von $H_{k}\left(S_{t+\tau}\right)$ gilt

$$
H_{k}^{\prime}\left(S_{t+\tau}\right)=\left(R_{t+\tau}^{k}\right)^{\prime}=\frac{k R_{t+\tau}^{k-1}}{S_{t+\tau}}
$$

Damit ist sowohl für $H_{k}$ als auch für $H_{k}^{\prime}$ die Auswertung an der Stelle $S_{t}$ gleich null:

$$
H_{k}\left(S_{t}\right)=H_{k}^{\prime}\left(S_{t}\right)=0
$$

Für die zweite Ableitung von $H_{k}$ gilt

$$
H_{k}^{\prime \prime}\left(S_{t+\tau}\right)=\left(R_{t+\tau}^{k}\right)^{\prime \prime}=\frac{k \cdot(k-1) R_{t+\tau}^{k-2}-k R_{t+\tau}^{k-1}}{S_{t+\tau}^{2}} .
$$

In Analogie zu Bakshi, Kapadia und Madan (2003) wird das Spanning-Argument aus Carr und Madan (2001) verwendet. ${ }^{13}$ Mit diesem lässt sich $H_{k}$ als 2-fach stetig differenzierbare Funktion in vier Bestandteile zerlegen:

$$
\begin{aligned}
H_{k}\left(S_{t+\tau}\right)= & H_{k}\left(S_{t}\right)+\left(S_{t+\tau}-S_{t}\right) H_{k}^{\prime}\left(S_{t}\right) \\
& +\int_{S_{t}}^{\infty} H_{k}^{\prime \prime}(K)\left(S_{t+\tau}-K\right)^{+} d K+\int_{0}^{S_{t}} H_{k}^{\prime \prime}(K)\left(K-S_{t+\tau}\right)^{+} d K \\
= & \int_{S_{t}}^{\infty} H_{k}^{\prime \prime}(K)\left(S_{t+\tau}-K\right)^{+} d K+\int_{0}^{S_{t}} H_{k}^{\prime \prime}(K)\left(K-S_{t+\tau}\right)^{+} d K .
\end{aligned}
$$

Diskontierung und Erwartungswertbildung sowie die Anwendung des Satzes von Fubini liefern schließlich die erwarteten nicht-zentralen Momente der Log-Rendite Verteilung unter dem physischen Maß.

$$
\begin{aligned}
& E^{P}\left\{e^{-r \tau} H_{k}\left(S_{t+\tau}\right)\right\} \\
= & E^{P}\left\{e^{-r \tau} \int_{S_{t}}^{\infty} H_{k}^{\prime \prime}(K)\left(S_{t+\tau}-K\right)^{+} d K+e^{-r \tau} \int_{0}^{S_{t}} H_{k}^{\prime \prime}(K)\left(K-S_{t+\tau}\right)^{+} d K\right\}
\end{aligned}
$$

13 Es gelten weiterhin die Annahmen an $S_{t}$ aus Lemma 1. 


$$
\begin{aligned}
= & \int_{S_{t}}^{\infty} H_{k}^{\prime \prime}(K)\left\{\int_{0}^{\infty} e^{-r \tau}\left(S_{t+\tau}-K\right)^{+} P\left(d S_{t+\tau}\right)\right\} d K \\
& +\int_{S_{t}}^{\infty} H_{k}^{\prime \prime}(K)\left\{\int_{0}^{\infty} e^{-r \tau}\left(K-S_{t+\tau}\right)^{+} P\left(d S_{t+\tau}\right)\right\} d K \\
= & \int_{S_{t}}^{\infty} H_{k}^{\prime \prime}(K) C^{P}(t, \tau, K) d K+\int_{0}^{S_{t}} H_{k}^{\prime \prime}(K) P^{P}(t, \tau, K) d K .
\end{aligned}
$$

Der diskontierte nicht-zentrale physische $k$-te Moment der Log-Rendite lässt sich demnach durch Integration der zweiten Ableitung von $H_{k}$ über Out-of-the-Money Call- und Put-EDAP's darstellen. Für Anwendungen sind insbesondere die Werte für $k=2,3$ und 4 von Bedeutung, für welche sich die Bezeichnungen, Volatility- , Cubic- und QuarticKontrakt in der Literatur etabliert haben. ${ }^{14}$ Korrolar 1 zeigt, wie diese bestimmt werden.

Korrolar 1: Speziell für den Volatility- $V(t, \tau)$, Cubic- $W(t, \tau)$ und Quartic- $X(t, \tau)$ -Kontrakt gilt

$$
\begin{aligned}
V(t, \tau)= & \int_{S_{t}}^{\infty} \frac{2\left(1-\log \left(\frac{K}{S_{t}}\right)\right)}{K^{2}} C^{P}(t, \tau, K) d K \\
& +\int_{0}^{S_{t}} \frac{2\left(1+\log \left(\frac{S_{t}}{K}\right)\right)}{K^{2}} P^{P}(t, \tau, K) d K, \\
W(t, \tau)= & \int_{S_{t}}^{\infty} \frac{6 \log \left(\frac{K}{S_{t}}\right)-3\left(\log \left(\frac{K}{S_{t}}\right)\right)^{2}}{K^{2}} C^{P}(t, \tau, K) d K \\
& -\int_{0}^{S_{t}} \frac{6 \log \left(\frac{S_{t}}{K}\right)+3\left(\log \left(\frac{S_{t}}{K}\right)\right)^{2}}{K^{2}} P^{P}(t, \tau, K) d K, \\
X(t, \tau)= & \int_{S_{t}}^{\infty} \frac{12\left(\log \left(\frac{K}{S_{t}}\right)\right)^{2}-4\left(\log \left(\frac{K}{S_{t}}\right)\right)^{3}}{K^{2}} C^{P}(t, \tau, K) d K \\
& +\int_{0}^{S_{t}} \frac{12\left(\log \left(\frac{S_{t}}{K}\right)\right)^{2}+4\left(\log \left(\frac{S_{t}}{K}\right)\right)^{3}}{K^{2}} P^{P}(t, \tau, K) d K .
\end{aligned}
$$

14 Vgl. Bakshi, Kapadia und Madan (2003). 
Beweis: Die Behauptung folgt unmittelbar aus Satz 1 und Gleichung (4.3) für $k=2,3$ und 4 .

Für den Übergang zu den zentralen Momenten der Verteilung der Log-Renditen, wie Varianz, Schiefe und Kurtosis, muss zusätzlich der Erwartungswert von $R_{t+\tau}$ unter dem physischen Maß bestimmt werden.

Falls der repräsentative Investor risikoneutral ist und somit die physische mit der risikoneutralen Dichte übereinstimmt, kann der Erwartungswert der Log-Rendite mit Hilfe einer Taylorentwicklung der Exponentialfunktion approximiert werden. ${ }^{15}$ Unter Verwendung der Tatsache, dass der Erwartungswert unter dem risikoneutralen Maß des Kurses des Assets $S_{t+\tau}$ gerade dem aktuellen aufgezinsten Kurs entspricht,

$$
E^{Q}\left\{\exp \left(R_{t+\tau}\right)\right\}=E^{Q}\left\{\exp \left(\log \left(\frac{S_{t+\tau}}{S_{t}}\right)\right)\right\}=\frac{E^{Q}\left\{S_{t+\tau}\right\}}{S_{t}}=e^{r \tau},
$$

erhält man aus der Talyorentwicklung folgende Approximation für den Erwartungswert:

$$
E^{Q}\left\{R_{t+\tau}\right\} \approx e^{r \tau}-1-\frac{1}{2} E^{Q}\left\{R_{t+\tau}^{2}\right\}-\frac{1}{6} E^{Q}\left\{R_{t+\tau}^{3}\right\}-\frac{1}{24} E^{Q}\left\{R_{t+\tau}^{4}\right\}
$$

Mit der präsentierten Methodik kann der Erwartungswert unter dem risikoneutralen Maß sogar exakt angegeben werden (vgl. Jiang und Tian (2005)). Hierzu sei das SpanningArgument aus Carr und Madan (2001) direkt auf die Log-Rendite $R_{t+\tau}$ angewandt und auf beiden Seiten der Gleichung der diskontierte risikoneutrale Erwartungswert gebildet. Es folgt

$$
E^{Q}\left\{R_{t+\tau}\right\}=e^{r \tau}-1-e^{r \tau} \int_{S_{t}}^{\infty} \frac{C(t, \tau, K)}{K^{2}} d K-e^{r \tau} \int_{0}^{S_{t}} \frac{P(t, \tau, K)}{K^{2}} d K .^{16}
$$

Für den Fall, dass $P \neq Q$ gilt, kann der Erwartungswert approximativ mit Hilfe der charakterisierenden Eigenschaft des repräsentativen Investors dargestellt werden. Der nächste Satz leitet die Approximation her: ${ }^{17}$

15 Vgl. Bakshi, Kapadia und Madan (2003).

16 Für die empirische Studie wurde die approximierte Version aus Bakshi, Kapadia und Madan (2003) verwendet.

17 Ein Ansatz zur exakten Bestimmung des Erwartungswertes $E^{P}\left\{R_{t+\tau}\right\}$ liefert das Auszahlungsprofil eines Portfolios, das aus einer long Call- und einer short Put-Position mit jeweiligen Ausübungspreis $S_{t}$ besteht. Dieses Portfolio dupliziert die Auszahlung des Assets $S_{t+\tau}$ verschoben um den Kurs $S_{t}$. Unter dem physischen Maß wird unter Verwendung der EDAP's der Erwartungswert des Portfolios durch $e^{r \tau} C^{P}\left(t, \tau, S_{t}\right)-e^{r \tau} P^{P}\left(t, \tau, S_{t}\right)$ gegeben, was wiederum mit dem Erwartungswert von $\frac{S_{t+\tau}}{S_{t}}$ übereinstimmt. Der Erwartungswert $E^{P}\left\{R_{t+\tau}\right\}$ der Log-Rendite wird nun in Analogie zu der exakten 
Satz 2: Sei $E^{P}\left\{R_{t+\tau}\right\}$ mit $\mu_{t, \tau}$ bezeichnet. Dann sind die Nullstellen des Polynoms

$$
\begin{aligned}
& {\left[\frac { d } { d \omega } \left(U\left(\omega\left(1+\mu_{t, \tau}\right)+(1-\omega) e^{r \tau}\right)\right.\right.} \\
& \quad+\frac{U^{(2)}\left(\omega\left(1+\mu_{t, \tau}\right)+(1-\omega) e^{r \tau}\right)}{2} E^{P}\left\{\left(R_{t+\tau}-\mu_{t, \tau}\right)^{2}\right\} \\
& \quad+\frac{U^{(3)}\left(\omega\left(1+\mu_{t, \tau}\right)+(1-\omega) e^{r \tau}\right)}{6} E^{P}\left\{\left(R_{t+\tau}-\mu_{t, \tau}\right)^{3}\right\} \\
& \left.\left.\quad+\frac{U^{(4)}\left(\omega\left(1+\mu_{t, \tau}\right)+(1-\omega) e^{r \tau}\right)}{24} E^{P}\left\{\left(R_{t+\tau}-\mu_{t, \tau}\right)^{4}\right\}\right)\right]_{\mid \omega=1}
\end{aligned}
$$

die möglichen (approximativen) Kandidaten für $\mu_{t, \tau}$.

Beweis: Im Falle eines Anlageuniversums von zwei Assets - einem risikolosen und einem riskanten - kann das Vermögen $W$ eines Portfolios mit einem Anteil von $\omega$ im riskanten Asset durch die Log-Rendite des riskanten Assets mit Hilfe einer Taylorentwicklung der Exponentialfunktion bis zur ersten Ordnung folgendermaßen approximiert werden:

$$
W=\omega e^{R_{t+\tau}}+(1-\omega) e^{r \tau} \approx \omega\left(1+R_{t+\tau}\right)+(1-\omega) e^{r \tau}
$$

Dementsprechend wird das erwartete Vermögen approximativ gegeben durch

$$
E^{P}\{W\} \approx \omega\left(1+\mu_{t, \tau}\right)+(1-\omega) e^{r \tau}
$$

Die charakterisierende Eigenschaft des repräsentativen Investors besagt für dieses Problem, dass das optimale Gewicht des riskanten Assets $\omega$ zu Beginn des Anlagehorizonts gleich 1 ist. Genauer gilt somit für die First-Order-Condition, dass

$$
\left[\frac{d}{d \omega} E^{P}\{U(\omega)\}\right]_{\mid \omega=1}=0
$$

Darstellung aus Jiang und Tian (2005) für den Erwartungswert unter dem risikoneutralen Maß gegeben durch

$$
E^{P}\left\{R_{t+\tau}\right\}=e^{r \tau} \frac{C^{P}\left(t, \tau, S_{t}\right)}{S_{t}}-e^{r \tau} \frac{P^{P}\left(t, \tau, S_{t}\right)}{S_{t}}-e^{r \tau} \int_{S_{t}}^{\infty} \frac{C^{P}(t, \tau, K)}{K^{2}} d K-e^{r \tau} \int_{0}^{S_{t}} \frac{P^{P}(t, \tau, K)}{K^{2}} d K .
$$

Für die empirische Studie wurde die (folgende) approximierte Version aus Satz 2 verwendet. 
Eine Taylorentwicklung der Nutzenfunktion bis zur vierten Ordnung ${ }^{18}$ und Erwartungswertbildung liefert folgende Approximation des Erwartungsnutzens:

$$
\begin{aligned}
E^{P}\{U(W)\} \approx & U\left(E^{P}\{W\}\right)+\frac{U^{(2)}\left(E^{P}\{W\}\right)}{2} \mu^{(2)} \\
& +\frac{U^{(3)}\left(E^{P}\{W\}\right)}{6} \mu^{(3)}+\frac{U^{(4)}\left(E^{P}\{W\}\right)}{24} \mu^{(4)}
\end{aligned}
$$

wobei $\mu^{(n)}$ den $n$-ten zentralen Moment von $W$ bezeichnet, d. h.

$$
\mu^{(n)}=E^{P}\left\{\left(W-E^{P}\{W\}\right)^{n}\right\} .
$$

Durch Einsetzen des approximierten erwarteten Vermögens und der Talyorentwicklung in die First-Order-Condition folgt die Behauptung.

Mit den Formeln aus Korollar 1 lassen sich nun bei bekannter Nutzenfunktion $U$ des repräsentativen Investors die höheren nicht-zentralen Momente von $R_{t+\tau}{ }^{19}$ bestimmen. Der risikolose Zinssatz ist bekannt. Es verbleibt nunmehr die Aufgabe, das Polynom aus Satz 2 in Abhängigkeit des Erwartungswertes von $R_{t+\tau}$ zu lösen. ${ }^{20}$

Die Nullstellen des Polynoms sind nach Satz 2 die möglichen Kandidaten für $E^{P}\left\{R_{t+\tau}\right\}$, welche unter ökonomischen Gesichtspunkten untersucht werden müssen. Als Resultat ergibt sich schließlich eine Approximation für den Erwartungswert $E^{P}\left\{R_{t+\tau}\right\} .{ }^{21}$ Hiermit können nun die zentralen Momente von $R_{t+\tau}$ bestimmt werden. Korrolar 2 zeigt die entsprechenden Formeln für Varianz, Schiefe und Kurtosis.

Korrolar 2: Die zentralen höheren Momente der Log-Renditeverteilung unter dem physischen Maß werden gegeben durch

$$
\begin{aligned}
\text { Varianz: } & e^{r \tau} V(t, \tau)-\mu_{t, \tau}^{2}, \\
\text { Schiefe: } & e^{r \tau} W(t, \tau)-3 e^{r \tau} V(t, \tau) \mu_{t, \tau}+2 \mu_{t, \tau}^{3}, \\
\text { Kurtosis: } & e^{r \tau} X(t, \tau)-4 e^{r \tau} W(t, \tau) \mu_{t, \tau}+6 e^{r \tau} V(t, \tau) \mu_{t, \tau}^{2}-3 \mu_{t, \tau}^{3},
\end{aligned}
$$

18 Vgl. Gleichung (2.3).

19 Man beachte, dass zur Bestimmung der nicht-zentralen Momente die entsprechenden Kontrakte aufgezinst werden müssen.

20 Für die empirische Studie wurden die Erwartungswerte $E^{P}\left\{\left(R_{t+\tau}-\mu_{t, \tau}\right)^{k}\right\}$ (vgl. Korrolar 2) ausmultipliziert und die Differentation nach $\omega$ für eine CRRA-Nutzenfunktion mit Hilfe von DERIVE durchgeführt. Das Polynom fünften Grades wurde abschließend in Abhängigkeit der nicht-zentralen Momente, des Zinssatzes und des Erwartungswertes dargestellt und die Nullstellen bestimmt (RPaket: polynom, base).

21 Z. B. Imaginärteil gleich null, extreme Werte, etc. 
wobei mit $V(t, \tau), W(t, \tau)$ und $X(t, \tau)$ der Volatility-, Cubic- bzw. Quartic-Kontrakt sowie mit $\mu_{t, \tau}$ der approximierte Erwartungswert bezeichnet ist.

Beweis: Die Behauptung folgt z. B. unmittelbar aus dem Pascal'schen Dreieck.

Zusammenfassend sind somit für die Varianz und die höheren Momente Schiefe und Kurtosis implizite modellfreie Momente unter dem physischen Maß hergeleitet worden. Bei bekannter Nutzenfunktion des repräsentativen Investors können diese aus der hergeleiteten Systematik ohne Verwendung numerischer Ableitungen bestimmt werden. Sie stellen eine direkte Erweiterung der modellfreien risikoneutralen Momente von Bakshi, Kapadia und Madan (2003) dar und können als Spezialfall, dass der repräsentative Investor risikoneutral ist, abgeleitet werden. Bevor jedoch die risikoadjustierten Momente im Rahmen der taktischen Asset Allokation zur Anwendung kommen, werden im folgenden Teil des Kapitels zunächst Wirkungen der Risikoadjustierung auf die impliziten Momente sowie die Prognosefähigkeit entsprechender realisierter Momente für den S\&P 500 untersucht.

\subsection{Daten}

Für den folgenden empirischen Teil des Kapitels werden europäische Optionen mit einer Laufzeit von einem Monat auf den S\&P 500 Index betrachtet. Die Optionsmarktdaten, insbesondere die zur Verfügung gestellten Volatilitätsoberflächen ${ }^{22}$ sowie entsprechende Schlusskurse stammen aus der Optionsdatenbank IvyDB von Optionmetrics. Diese Daten beziehen sich auf Optionen, die an der CBOE gehandelt werden. Der verfügbare Zeitraum umfasst Januar 1996 bis Oktober 2010. Als Stichtag für jeden Monat wird der erste Montag nach dem dritten Freitag verwendet. Dies entspricht dem ersten Handelstag nach dem Verfallstermin von Optionen an der CBOE und garantiert eine möglichst gute Approximation der Laufzeit der Optionen von einem Monat. Insgesamt liegen 177 Beobachtungszeitpunkte vor.

Als risikofreie Verzinsung wird der durch die IvyDB bereitgestellte Zinssatz $r$ genutzt. Die Zinsstruktur wird hierbei aus dem BBA Libor Zinssatz und den Settlement Preisen von CME Eurodollar Futures berechnet. ${ }^{23}$

22 Vgl. Methodik Kapitel 1. Die Volatilitätsoberflächen liegen um Dividenden bereinigt in der IvyDB vor. Da in dieser Studie die höheren Momente der Verteilung untersucht werden, wird auf eine weitere Berücksichtigung der Dividendenrendite verzichtet.

23 Vgl. Optionmetrics Handbuch. 
Zur Bestimmung realisierter Momente werden Intraday Daten aus der "Historical Market Data" Datenbank von Pi Trading ${ }^{24}$ verwendet. Die Daten liegen auf minütlicher Basis für den gesamten Zeitraum vor.

\subsection{Methodik und Design der empirischen Studie}

Für die Berechnung der EDAP's wird ein Kontinuum von Out-of-the-Money Put- und Call-Optionen bzgl. des Ausübungspreises sowie ein entsprechendes Kontinuum von Digitaloptionen benötigt. Zur Konstruktion wird ein analoger Vorgang wie im vorherigen Kapitel $^{25}$ verwendet. Diese Methodik liefert die benötigten Put- und Call-Optionen. Zur Bestimmung der Werte der Digitaloptionen wird die Annahme getroffen, dass die aus Putund Call-Optionen vorhandene Volatilitätsoberfläche zur Bewertung verwendet werden kann. Mit der Formel für Digitaloptionen ${ }^{26}$ lässt sich schließlich das Kontinuum bestimmen.

Die durchzuführenden Integrationen bzgl. der Optionspreise werden mit Hilfe numerischer Integration auf dem Intervall $\left[0 ; 3 \cdot S_{t}\right]$ mit Hilfe von 1000 Stützstellen mit der Trapez-Regel durchgeführt.

Um realisierte Momente aus Intraday-Daten zu erhalten, wird die Methodik aus Amaya, Christoffersen, Jacobs und Vasquez (2011) übernommen. Diese Methodik erweitert das für die realisierte Volatilität konstruierte Verfahren auf die höheren Momente Schiefe und Kurtosis aus Andersen und Bollerslev (1998) und Andersen, Bollerslev, Diebold und Labys (2003).

Hierzu seien zunächst aus den Intraday-Kursen die Log-Intraday Returns gebildet. Unter der Annahme, dass Erwartungswert und Dividenden ${ }^{27}$ bei Intraday-Daten zu vernachlässigen sind, wird die realisierte Varianz des Tages $t$ gegeben durch

$$
\operatorname{RDV}_{\operatorname{lar}}=\sum_{i=1}^{N} r_{t, i}^{2}
$$

wobei $r_{t, i}$ den $i$-ten Log-Intraday Return und $N$ die absolute Anzahl von Beobachtungen des Tages angibt.

\footnotetext{
$24 \quad$ Siehe pitrading.com.

25 Vgl. Methodik Kapitel 3.

$26 D(t, \tau, K)=e^{-r \tau} \Phi\left(d_{2}\right)$ (abgeleitet aus der Black-Scholes Formel für den Call).

27 Vgl. Jiang und Tian (2005).
} 
Die standardisierte Intraday-Schiefe und -Kurtosis können analog formuliert werden

$$
\operatorname{RDSkew}_{t}=\frac{\sqrt{N} \sum_{i=1}^{N} r_{t, i}^{3}}{R D V a r_{t}^{3 / 2}}, \quad \text { RDKurt }_{t}=\frac{N \sum_{i=1}^{N} r_{t, i}^{4}}{R D V a r_{t}^{2}}
$$

Schließlich werden mittels Durchschnittsbildung aus den gewonnenen Momenten jedes Tages die entsprechenden Momente für den betrachteten Horizont bestimmt. Für die Volatilität muss der Wert adäquat skaliert, werden z. B. mit $\sqrt{252}$ annualisiert. Die durchschnittliche Schiefe und Kurtosis können hingegen als standardisierte Werte direkt verwendet werden.

In den folgenden Abschnitten werden repräsentative Investoren mit CRRA Nutzenfunktionen und Risikoaversionsparametern 0, 2, 4, 6, 8, 10 und 12 betrachtet und die entsprechenden risikoadjustierten impliziten Momente gebildet. Die Momente für den Risikoaversionsparameter 0, also im Fall, dass der repräsentative Investor risikoneutral ist, entsprechen hierbei den "rein" impliziten Momenten aus Bakshi, Kapadia und Madan (2003). Die Nutzenfunktionen werden im Zeitablauf als konstant angenommen. Es wird also davon ausgegangen, dass der jeweilige repräsentative Investor keine zeitvariable Risikoaversion hat. Das Design der empirischen Studie entspricht einer komparativ-statischen Analyse.

\subsection{Konsequenzen aus der Risikoadjustierung}

In diesem Abschnitt wird die Wirkung der Risikoadjustierung auf die impliziten Momente untersucht. Zunächst wird hierzu der Einfluss auf die EDAP's aus Lemma 1 betrachtet. Es folgt eine Diskussion zu der abgeleiteten Markt-Risikoprämie aus Satz 2. Abschließend wird die Wirkung auf die impliziten Momente untersucht. Zum einen wird auf den Vergleich zwischen der exakt hergeleiteten Risikoadjustierung und einer Approximation im Sinne von Bakshi, Kapadia und Madan (2003), zum anderen auf die Differenz zwischen risikoadjustiertem und risikoneutralem Moment mit besonderem Augenmerk auf die zeitliche Struktur eingegangen.

\subsubsection{Wirkung der Risikoadjustierung auf OTM-Optionen}

Abbildung 4.1 zeigt den Einfluss der Risikoadjustierung auf die EDAP's, wobei die linke Hälfte sich auf den Put und die rechte Hälfte auf den Call bezieht. Es lässt sich beobachten, dass mit steigender Risikoaversion des repräsentativen Investors die EDAP's für den Put 


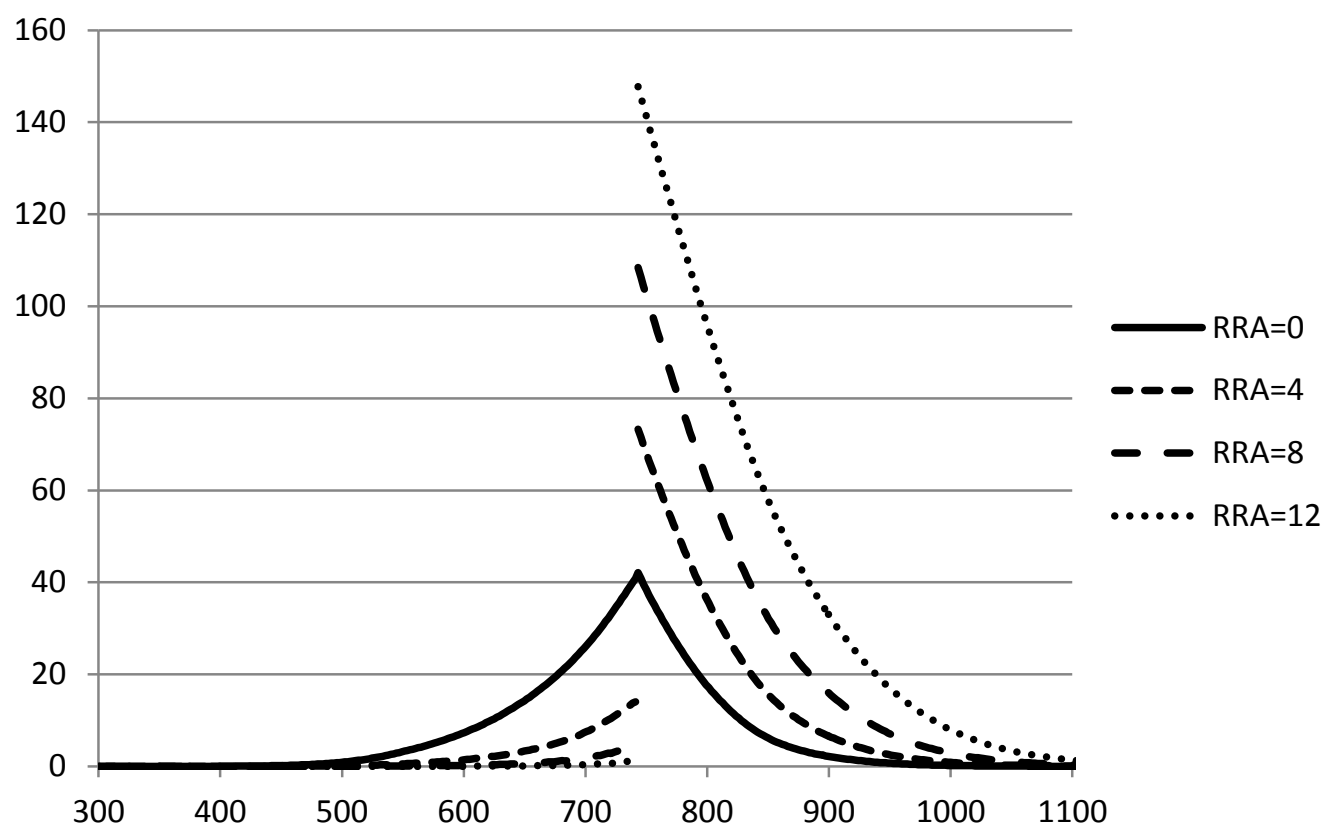

Abbildung 4.1: Diese Abbildung zeigt den Einfluss der Risikoadjustierung für repräsentative Investoren mit CRRA Nutzenfunktionen auf die EDAP's in Abhängigkeit des Ausübungspreises. Auf $[0 ; 743,33]$ sind die EDAPS's für den Put, auf $[743,33 ; 1100]$ die EDAPS's für den Call abgebildet. Die Kurven beziehen sich auf Daten von Optionen auf den S\&P 500 vom 23.02.2009 mit einer Laufzeit von einem Monat. Der Kurs vom 23.02.2009 des S\&P 500 beträgt 743,33.

sinken und die EDAP's für den Call steigen. Dies ist im Einklang mit der Intuition, dass durch die Risikoaversion insbesondere der Erwartungswert des Kurses gesteuert wird.

Ein risikoaverser repräsentativer Investor verlangt für die Kompensation des eingegangenen Risikos gegenüber der risikolosen Anlage eine höhere erwartete Rendite. Dies hat zur Folge, dass der EDAP für den Put sinkt, da dieser weniger wahrscheinlich ausgeübt wird. Für den Call ergibt sich für die Wertsteigerung eine analoge Argumentation, da dieser nun mit einer höheren Wahrscheinlichkeit ins Geld kommt.

Für die Marktpreise $(\mathrm{RRA}=0)$ wird ungefähr im aktuellen Kurs 743,33, genauer im risikoneutralen Erwartungswert $S_{t} \cdot(1+r f)=743,33 \cdot(1+0,04 \%)=743,66,{ }^{28}$ die Gleichheit zwischen Call und Put Wert erreicht. Für die risikoadjustierten EDAP's ist dieser Punkt in der Abbildung nach rechts verlagert, und zwar an die Stelle, an welcher der Ausübungsübungspreis mit dem erwarteten Kurs übereinstimmt. 


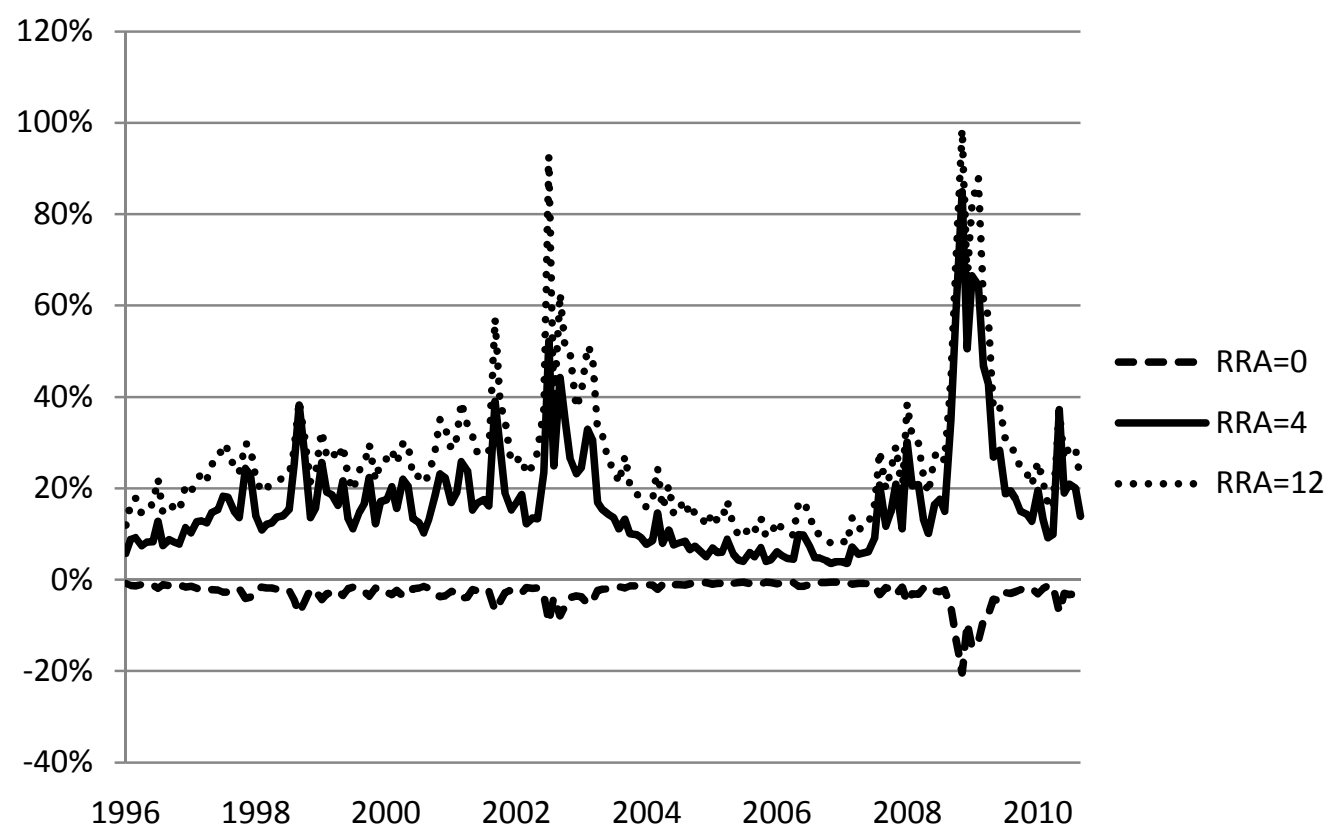

Abbildung 4.2: Diese Abbildung zeigt die approximierten Excess Returns für unterschiedliche Risikoaversionen von Investoren mit CRRA Nutzenfunktionen. RRA $=0$ gibt den impliziten (nicht-risikoadjustierten) Wert an. Die Werte sind annualisiert und beziehen sich auf Daten von Optionen auf den S\&P 500 mit einer Laufzeit von einem Monat. Der Zeitraum beträgt Januar 1996 bis Oktober 2010.

\subsubsection{Die Markt-Risikoprämie}

Tabelle 4.2: Deskriptive Statistiken für die approximierten (impliziten) Excess Returns

\begin{tabular}{cccccc}
\hline & ERW & STD & $25 \%$ & MED & $75 \%$ \\
\hline 0 & $-0,027$ & 0,026 & $-0,031$ & $-0,021$ & $-0,012$ \\
2 & 0,101 & 0,090 & 0,048 & 0,079 & 0,119 \\
4 & 0,167 & 0,123 & 0,088 & 0,138 & 0,200 \\
6 & 0,208 & 0,132 & 0,118 & 0,181 & 0,249 \\
8 & 0,233 & 0,136 & 0,142 & 0,212 & 0,279 \\
10 & 0,249 & 0,140 & 0,156 & 0,230 & 0,296 \\
12 & 0,261 & 0,150 & 0,165 & 0,234 & 0,294 \\
\hline
\end{tabular}

Diese Tabelle zeigt deskriptive Statistiken für die approximierten Excess Returns für unterschiedliche Risikoaversionen von Investoren mit CRRA Nutzenfunktionen. RRA $=0$ gibt den impliziten (nichtrisikoadjustierten) Wert an. Die Werte sind annualisiert und beziehen sich auf Daten von Optionen auf den S\&P 500 mit einer Laufzeit von einem Monat. Der Zeitraum beträgt Januar 1996 bis Oktober 2010.

Von besonderem Interesse sind die aus dem Modell abgeleiteten (erwarteten) Überrenditen des S\&P 500. Diese können als implizite Markt-Risikoprämie interpretiert werden. Abbildung 4.2 zeigt die Excess Returns für verschiedene Risikoaversionen im Zeitablauf,

28 Dividenden werden nicht berücksichtigt. 
Tabelle 4.2 entsprechende deskriptive Statistiken. Es lässt sich erkennen, dass die geschätzten Excess Returns für repräsentative Investoren mit positiver Risikoaversion größer als null sind.

Des Weiteren steigen die erwarteten Markt-Risikoprämien sowie die Standardabweichungen mit der Risikoaversion des Investors an. Dies ist im Einklang mit der Intuition, dass ein risikoaverserer Investor für dasselbe Risiko eine höhere erwartete Rendite bzw. Kompensation verlangt. Der Vergleich aus Erwartungswert und Median zeigt eine asymmetrische rechtsschiefe Verteilung der erwarteten Prämien.

In der Abbildung sind die "Dot-Com-Krise" und die Finanzmarktkrise klar zu erkennen. In diesen Perioden steigt die "verlangte" erwartete Risikoprämie aufgrund des erhöhten impliziten Risikos sprunghaft an. Diese Werte erklären die rechtsschiefe, asymmetrische Verteilung sowie die steigenden Werte in der Standardabweichung. Je risikoaverser der repräsentative Investor ist, desto überzeichneter tritt dieser Effekt auf. Dass implizite Risikoprämien sehr sensibel auf verändertes Risiko reagieren, zeigen auch die Ergebnisse von Duan und Zhang (2010). In den Krisen schätzen die Autoren Risikoprämien von bis zu $208 \%$ p.a. ("Dot-Com-Krise") und $539 \%$ p.a. (Finanzmarktkrise).

\subsubsection{Wirkung der Risikoadjustierung auf Varianz, Schiefe und Kurtosis}

In diesem Abschnitt wird die Wirkung der Risikoadjustierung auf die Momente der LogRendite Verteilung untersucht. Hierfür wird zunächst die Differenz zwischen dem risikoadjustierten und dem risikoneutralen Moment gebildet. Abbildung 4.3 zeigt die Differenzen im Zeitablauf. Tabelle 4.3 liefert entsprechende deskriptive Statistiken.

Für die Volatilität lassen sich hinsichtlich der Risikoaversion nur marginale Differenzen im Zeitablauf erkennen. Dies ist im Einklang mit dem im vorherigen Kapitel diskutierten Beispiel, in dem die physische mit der risikoneutralen Volatilität übereinstimmt und somit die Risikoaffinität insbesondere die erwartete Rendite steuert. In ruhigen Marktphasen lässt sich ein negativer Spread zwischen den Momenten beobachten, der jedoch bei steigender Risikoaversion nicht stark variiert. Die negative Differenz deutet auf eine Reduktion der Varianz-Risikoprämie hin. In Krisenphasen hingegen divergiert der Spread und es lassen sich Unterschiede zwischen den Risikoaversionen erkennen. Daraus ist ableitbar, dass gerade in Zeiten hoher Marktunsicherheit die Einschätzung zukünftiger Volatilitäten durch unterschiedliche Investoren voneinander abweicht. Insbesondere im Fall stark risikoaverser Investoren $(\mathrm{RRA}=12)$ besteht eine Differenz von über 20 (p.a.) Prozentpunkte ("Dot-Com-Krise") bzw. 27,5 (p.a.) Prozentpunkte (Finanzmarktkrise) zwischen dem risikoadjustierten und dem impliziten Moment. In Phasen von hohen Marktturbulen- 


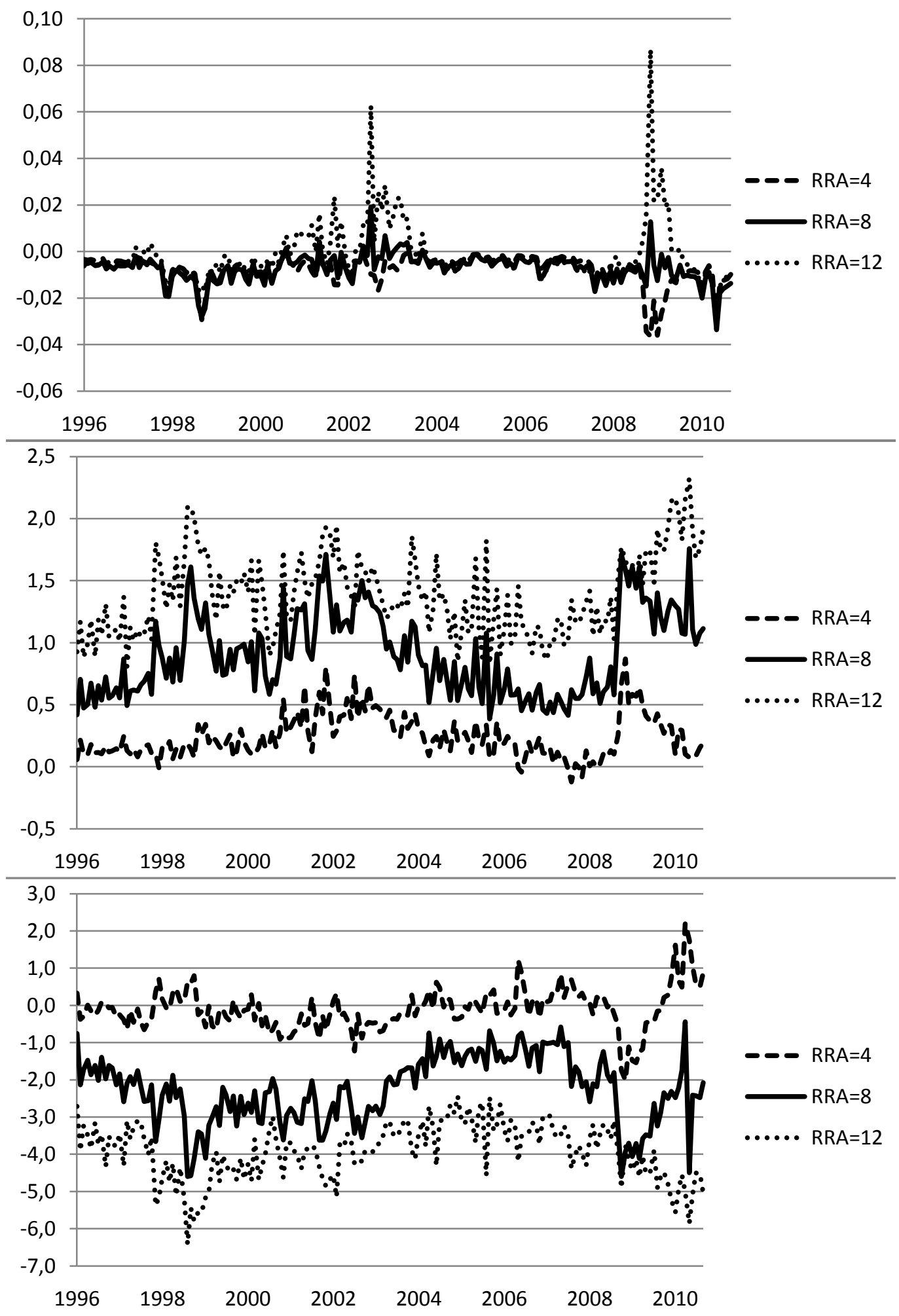

Abbildung 4.3: Diese Abbildung zeigt die Differenz zwischen der (nicht annualisierten) risikoadjustierten (CRRA Nutzenfunktionen, Risikoaversion 4, 8 und 12) Volatilität, (standardisierte) Schiefe und Kurtosis und den impliziten Momenten. Die Werte beziehen sich auf Daten von Optionen auf den S\&P 500 mit einer Laufzeit von einem Monat. Der Zeitraum beträgt Januar 1996 bis Oktober 2010. 
zen überschätzen stark risikoaverse Investoren das Marktrisiko und neigen somit zu einer überzeichneten Prognose der zukünftigen realisierten Volatilität. Dies drückt sich auch in der impliziten Markt-Risikoprämie aus dem vorherigen Abschnitt aus.

In den Abbildungen für die Schiefe und Kurtosis lassen sich im Gegensatz zu der Volatilität systematische Unterschiede zwischen den unterschiedlichen Risikoaversionen erkennen. Im Einzelnen wird eine jeweilige Verschiebung in Abhängigkeit der Risikoaversion festgestellt. Für die Differenz in der Schiefe zeigt sich ein steigender, für die Differenz in der Kurtosis ein sinkender Trend. Diese Verschiebungen lassen sich aus den Präferenzen der repräsentativen Investoren ableiten und erklären. Die Risikoaversionen der Investoren werden allesamt als positiv angenommen, dies bedeutet, dass die Investoren eine Präferenz für positive Schiefe und eine Abneigung gegen Kurtosis-Risiken haben (vgl. Kapitel 2).

Der spezifische repräsentative Investor, welcher die physische Verteilung am Markt determiniert, wird die Momente der Renditeverteilung so wählen, dass im Gleichgewichtszustand die First-Order Condition erfüllt ist (vgl. Beweis zu Satz 2). Da in diesem Rahmen keine Verteilungsannahme an die Renditeverteilung getroffen wurde, ist im Gegensatz zu dem Fall, dass ein log-normalverteilter Kurs vorliegt, die Risikoadjustierung auch in der Schiefe und Kurtosis wirksam. Es gilt, je risikoaverser der Investor ist, desto höher ist die Schiefe unter dem physischen Maß. Eine analoge Argumentation gilt für die Kurtosis. Der repräsentative Investor meidet hohe Tail-Risiken und reduziert daher die Kurtosis.

Des Weiteren zeigen die Abbildungen für Schiefe und Kurtosis im deutlichen Gegensatz zu der Zeitreihe für die Volatilität einen nicht-konstanten, zeitvariablen Anteil am Spread zwischen den Momenten. Insbesondere an der Schiefe lässt sich erkennen, dass in unruhigen Marktphasen die Differenz divergiert, in ruhigen Phasen hingegen der Spread wieder rückläufig ist. Dies kann dahingehend interpretiert werden, dass in unruhigen Marktphasen der repräsentative Investor eine größere Kompensation für die Übernahme von Risiken aus höheren Momenten verlangt als in ruhigen Marktphasen. 
Tabelle 4.3: Erwartungswerte und Standardabweichungen für die Differenz aus risikoadjustiertem und implizitem Moment

\begin{tabular}{|c|c|c|c|c|c|c|}
\hline & ERW & STD & ERW & STD & ERW & STD \\
\hline & \multicolumn{2}{|c|}{ Volatilität } & \multicolumn{2}{|c|}{ Schiefe } & \multicolumn{2}{|c|}{ Kurtosis } \\
\hline 2 & $-0,017$ & 0,016 & $-0,009$ & 0,095 & 0,399 & 0,395 \\
\hline 4 & $-0,026$ & 0,022 & 0,238 & 0,174 & $-0,108$ & 0,566 \\
\hline 6 & $-0,029$ & 0,020 & 0,576 & 0,281 & $-1,137$ & 0,803 \\
\hline 8 & $-0,025$ & 0,021 & 0,908 & 0,333 & $-2,264$ & 0,903 \\
\hline 10 & $-0,016$ & 0,030 & 1,185 & 0,339 & $-3,219$ & 0,852 \\
\hline 12 & $-0,005$ & 0,043 & 1,391 & 0,326 & $-3,927$ & 0,755 \\
\hline
\end{tabular}

Diese Tabelle zeigt die Erwartungswerte und Standardabweichungen für die Differenz aus risikoadjustierten (CRRA Nutzenfunktionen) und impliziten Momenten. Die Volatilitäten sind annualisiert. Schiefen und Kurtosin sind standardisiert. Die Werte beziehen sich auf Daten von Optionen auf den S\&P 500 mit einer Laufzeit von einem Monat. Der Zeitraum beträgt Januar 1996 bis Oktober 2010.

Tabelle 4.3 zeigt deskriptive Statistiken für die Differenz aus dem Spread zwischen dem risikoadjustierten und dem risikoneutralen Moment. Für die Volatilität ergeben sich im Einklang mit Abbildung 4.3 leicht negative Differenzen zwischen dem risikoneutralen und dem physischen Moment. Für den repräsentativen Investor mit Risikoaversion 12 ist die Differenz jedoch auf einem 10\%-Niveau statistisch nicht mehr signifikant von null verschieden. Für die Risikoaversion 6 ist die Differenz (absolut gemessen) am größten und lässt in diesem Fall die größte Reduktion der Varianz-Risikoprämie vermuten. Dies kommt auch in einer geringen Standardabweichung zum Ausdruck.

Für Schiefe und Kurtosis ist die in der Abbildung 4.3 beobachtete systematische Verschiebung zu verifizieren. Für steigende Risikoaversionen steigt die Schiefe, die Kurtosis fällt. Lediglich für die Risikoaversion 2 kann kein auf einem 10\%-Niveau statistisch signifikanter Unterschied für die Schiefe festgestellt werden.

Abschließend ist festzuhalten, dass durch die Risikoadjustierung für Risikoaversionen größer als 8 approximativ folgende Wirkungen auf die höheren Momente der Verteilung zu beobachten sind. Die Volatilität bleibt annähernd unverändert, die Schiefe steigt und die Kurtosis sinkt. Dies bedeutet im Vergleich zu der Normalverteilung eine rechtsschiefe und abgeflachte Verteilung.

Zur weiteren Untersuchung der zeitlichen Struktur des Spreads aus Abbildung 4.3 wird eine approximative Darstellung in Abhängigkeit der risikoneutralen Momente und der Risikoaversion des repräsentativen Investors erarbeitet. Bakshi, Kapadia und Madan (2003) leiten mit Hilfe der Eigenschaften der momenterzeugenden Funktion der Log-Rendite Verteilung eine approximative Beziehung zwischen den risikoneutralen und physischen Momenten der Verteilung her. In der Literatur wurde diese Approximation unter ande- 
rem dazu verwendet, Volatilitäts-Spreads (vgl. Bakshi und Madan (2006)) oder "implizite" Markt-Risikoprämien (vgl. Duan und Zhang (2010)) zu studieren. Für diese Analyse wird für die umgekehrte Richtung, d. h. der physische Moment wird in Abhängigkeit der risikoneutralen Momente dargestellt, die Approximation im Folgenden kurz reproduziert.

\section{Herleitung der Approximation}

Da eine konstante Verschiebung einer Zufallsvariablen keinen Einfluss auf die höheren Momente der Verteilung hat, sei o. B. d. A. $E^{Q}\{R\}=0$. Für einen repräsentativen Investor mit CARA Nutzenfunktion und Risikoaversion $\gamma$ besteht folgende Beziehung zwischen den Dichten von $Q$ und $P:{ }^{29}$

$$
p(R)=\frac{e^{\gamma R} \cdot q(R)}{\int e^{\gamma R} \cdot q(R) d R}
$$

Sei $\mathcal{M}[\lambda]=\int e^{\lambda R} q(R) d R$ die momenterzeugende Funktion von $Q$ und $\overline{\mathcal{M}}[\lambda]$ diejenige von $P$.

Dann gilt die Beziehung

$$
\overline{\mathcal{M}}[\lambda]=\int e^{\lambda R} p(R) d R=\frac{\int e^{\lambda R} e^{\gamma R} q(R) d R}{\int e^{\gamma R} q(R) d R}=\frac{\mathcal{M}[\lambda+\gamma]}{\mathcal{M}[\gamma]} .
$$

Die momenterzeugende Funktion von $P$ kann somit durch die von $Q$ ausgedrückt werden. Eine Taylorentwicklung von $\mathcal{M}[\lambda]$ um $E^{Q}\{R\}=0$ liefert

$$
\mathcal{M}[\lambda]=1+\frac{\lambda^{2}}{2} E^{Q}\left\{R^{2}\right\}+\frac{\lambda^{3}}{6} E^{Q}\left\{R^{3}\right\}+\frac{\lambda^{4}}{24} E^{Q}\left\{R^{4}\right\}+\frac{\lambda^{5}}{120} E^{Q}\left\{R^{5}\right\}+\frac{\lambda^{6}}{720} E^{Q}\left\{R^{6}\right\} .
$$

Aus der Taylorentwicklung folgt, dass $\mathcal{M}[\gamma]=1+\frac{\gamma^{2}}{2} E^{Q}\left\{R^{2}\right\}+o\left(\gamma^{3}\right)$ ist. Die Ableitungen nach $\lambda$ liefern nun folgendes Gleichungssystem:

$$
\begin{aligned}
& \frac{d}{d \lambda} \mathcal{M}[\lambda]=\lambda E^{Q}\left\{R^{2}\right\}+\frac{\lambda^{2}}{2} E^{Q}\left\{R^{3}\right\}+\frac{\lambda^{3}}{6} E^{Q}\left\{R^{4}\right\}+\frac{\lambda^{4}}{24} E^{Q}\left\{R^{5}\right\}+\frac{\lambda^{5}}{120} E^{Q}\left\{R^{6}\right\} \\
& \frac{d^{2}}{d \lambda^{2}} \mathcal{M}[\lambda]=E^{Q}\left\{R^{2}\right\}+\lambda E^{Q}\left\{R^{3}\right\}+\frac{\lambda^{2}}{2} E^{Q}\left\{R^{4}\right\}+\frac{\lambda^{3}}{6} E^{Q}\left\{R^{5}\right\}+\frac{\lambda^{4}}{24} E^{Q}\left\{R^{6}\right\} \\
& \frac{d^{3}}{d \lambda^{3}} \mathcal{M}[\lambda]=E^{Q}\left\{R^{3}\right\}+\lambda E^{Q}\left\{R^{4}\right\}+\frac{\lambda^{2}}{2} E^{Q}\left\{R^{5}\right\}+\frac{\lambda^{3}}{6} E^{Q}\left\{R^{6}\right\}
\end{aligned}
$$

29 In diesem Fall wird die Renditeverteilung und nicht die Verteilung des Kurses transformiert. Es gilt, dass eine Risikoadjustierung der Renditeverteilung mit Hilfe einer CARA Nutzenfunktion der Risikoadjustierung der Verteilung des Kurses mit einer CRRA Nutzenfunktion entspricht. Um diesen Unterschied zu berücksichtigen, wird für die empirischen Auswertungen in die Approximationen die Risikoaversion $\gamma-1$ anstatt $\gamma$ eingesetzt. Dieser Zusammenhang folgt aus einer Substitution im Sinne von (3.2) für die Log-Rendite. 


$$
\frac{d^{4}}{d \lambda^{4}} \mathcal{M}[\lambda]=E^{Q}\left\{R^{4}\right\}+\lambda E^{Q}\left\{R^{5}\right\}+\frac{\lambda^{2}}{2} E^{Q}\left\{R^{6}\right\}
$$

Bis zur zweiten Ordnung von $\gamma$ ergibt sich für die nicht-zentralen Momente unter $P$ durch Einsetzen von $\lambda=0$ folgende rekursive Approximation durch die Momente unter Q:

$$
\begin{aligned}
& E^{P}\{R\} \approx\left(1+\frac{\gamma^{2}}{2} E^{Q}\left\{R^{2}\right\}\right)^{-1}\left(E^{Q}\{R\}+\gamma E^{Q}\left\{R^{2}\right\}+\frac{\gamma^{2}}{2} E^{Q}\left\{R^{3}\right\}\right) \\
& E^{P}\left\{R^{2}\right\} \approx\left(1+\frac{\gamma^{2}}{2} E^{Q}\left\{R^{2}\right\}\right)^{-1}\left(E^{Q}\left\{R^{2}\right\}+\gamma E^{Q}\left\{R^{3}\right\}+\frac{\gamma^{2}}{2} E^{Q}\left\{R^{4}\right\}\right) \\
& E^{P}\left\{R^{3}\right\} \approx\left(1+\frac{\gamma^{2}}{2} E^{Q}\left\{R^{2}\right\}\right)^{-1}\left(E^{Q}\left\{R^{3}\right\}+\gamma E^{Q}\left\{R^{4}\right\}+\frac{\gamma^{2}}{2} E^{Q}\left\{R^{5}\right\}\right) \\
& E^{P}\left\{R^{4}\right\} \approx\left(1+\frac{\gamma^{2}}{2} E^{Q}\left\{R^{2}\right\}\right)^{-1}\left(E^{Q}\left\{R^{4}\right\}+\gamma E^{Q}\left\{R^{5}\right\}+\frac{\gamma^{2}}{2} E^{Q}\left\{R^{6}\right\}\right) .
\end{aligned}
$$

Eine Polynomdivision liefert bis zur zweiten Ordnung von $\gamma$

$$
\begin{aligned}
& E^{P}\{R\} \approx E^{Q}\{R\}+\gamma E^{Q}\left\{R^{2}\right\}+\frac{\gamma^{2}}{2} E^{Q}\left\{R^{3}\right\} \\
& E^{P}\left\{R^{2}\right\} \approx E^{Q}\left\{R^{2}\right\}+\gamma E^{Q}\left\{R^{3}\right\}+\frac{\gamma^{2}}{2}\left(E^{Q}\left\{R^{4}\right\}-E^{Q}\left\{R^{2}\right\}\right) \\
& E^{P}\left\{R^{3}\right\} \approx E^{Q}\left\{R^{3}\right\}+\gamma E^{Q}\left\{R^{4}\right\}+\frac{\gamma^{2}}{2}\left(E^{Q}\left\{R^{5}\right\}-E^{Q}\left\{R^{2}\right\} E^{Q}\left\{R^{3}\right\}\right) \\
& E^{P}\left\{R^{4}\right\} \approx E^{Q}\left\{R^{4}\right\}+\gamma E^{Q}\left\{R^{5}\right\}+\frac{\gamma^{2}}{2}\left(E^{Q}\left\{R^{6}\right\}-E^{Q}\left\{R^{2}\right\} E^{Q}\left\{R^{4}\right\}\right) .
\end{aligned}
$$

Varianz, Schiefe und Kurtosis unter $P$ können nun durch die Momente von $Q$ bis zur zweiten Ordnung von $\gamma$ approximiert werden. Es ergeben sich folgende Darstellungen:

Für die Varianz:

$$
\begin{aligned}
V A R^{P}(R)= & E^{P}\left\{R^{2}\right\}-\left(E^{P}\{R\}\right)^{2} \\
\approx & E^{Q}\left\{R^{2}\right\}+\gamma E^{Q}\left\{R^{3}\right\}+\frac{\gamma^{2}}{2}\left(E^{Q}\left\{R^{4}\right\}-3 E^{Q}\left\{R^{2}\right\}^{2}\right) \\
\approx & V A R^{Q}(R)+\gamma S K E W^{Q}(R) V A R^{Q}(R)^{3 / 2} \\
& +\frac{\gamma^{2}}{2}\left(K U R T^{Q}(R)-3\right) V A R^{Q}(R)^{2}
\end{aligned}
$$

Für die Schiefe:

$$
S K E W^{P}(R)=\frac{E^{P}\left\{\left(R-E^{P}[R]\right)^{3}\right\}}{V A R^{P}(R)^{3 / 2}}
$$




$$
\begin{aligned}
= & \frac{E^{P}\left\{R^{3}\right\}-3 E^{P}\{R\} E^{P}\left\{R^{2}\right\}+2\left(E^{P}\{R\}\right)^{3}}{V A R^{P}(R)^{3 / 2}} \\
\approx & \frac{E^{Q}\left\{R^{3}\right\}+\gamma\left(E^{Q}\left\{R^{4}\right\}-3 \gamma E^{Q}\left\{R^{2}\right\}^{2}\right)}{E^{Q}\left\{R^{2}\right\}^{3 / 2}} \\
& +\frac{\frac{\gamma^{2}}{2}\left(E^{Q}\left\{R^{5}\right\}-10 E^{Q}\left\{R^{3}\right\} E^{Q}\left\{R^{2}\right\}\right)}{E^{Q}\left\{R^{2}\right\}^{3 / 2}} \\
\approx & S K E W^{Q}(R)+\gamma\left(K U R T^{Q}(R)-3\right) V A R^{Q}(R)^{1 / 2} \\
& +\frac{\gamma^{2}}{2}\left(P K E W^{Q}(R)-10 \cdot S K E W^{Q}(R)\right) V A R^{Q}(R)
\end{aligned}
$$

Für die Kurtosis:

$$
\begin{aligned}
K U R T^{P}(R)= & \frac{E^{P}\left\{\left(R-E^{P}\{R\}\right)^{4}\right\}}{V A R^{P}(R)^{2}} \\
= & \frac{E^{P}\left\{R^{4}\right\}-4 E^{P}\{R\} E^{P}\left\{R^{3}\right\}+6 E^{P}\{R\}^{2} E^{P}\left\{R^{2}\right\}-3 E^{P}\{R\}^{4}}{V A R^{P}(R)^{2}} \\
\approx & \frac{E^{Q}\left\{R^{4}\right\}+\gamma\left(E^{Q}\left\{R^{5}\right\}-4 E^{Q}\left\{R^{2}\right\} E^{Q}\left\{R^{3}\right\}\right)}{E^{Q}\left\{R^{2}\right\}^{2}} \\
& +\frac{\frac{\gamma^{2}}{2}\left(E^{Q}\left\{R^{6}\right\}-9 E^{Q}\left\{R^{4}\right\} E^{Q}\left\{R^{2}\right\}-4 E^{Q}\left\{R^{3}\right\}^{2}+12 E^{Q}\left\{R^{2}\right\}^{3}\right)}{E^{Q}\left\{R^{2}\right\}^{2}} \\
\approx & K U R T^{Q}(R)+\gamma\left(P K E W^{Q}(R)-4 \cdot S K E W^{Q}(R)\right) V A R^{Q}(R)^{1 / 2} \\
& +\frac{\gamma^{2}}{2}\left(H E X I C^{Q}(R)-9 \cdot K U R T^{Q}(R)\right. \\
& \left.-4 \cdot S K E W^{Q}(R)^{2}+12\right) V A R^{Q}(R),
\end{aligned}
$$

wobei mit $P K E W^{Q}(R)$ der fünfte, mit $V A R^{Q}(R)^{5 / 2}$ normierte, und mit $\operatorname{HEXIC}(R)$ der sechste, mit $V A R^{Q}(R)^{3}$ normierte, zentrale Moment bezeichnet wird.

Zusammenfassend können die Differenzen für Varianz, Schiefe und Kurtosis zwischen dem risikoadjustierten und dem risikoneutralen Moment bis zur ersten und zweiten Ordnung von $\gamma$ folgendermaßen approximiert werden. ${ }^{30}$

30 Für die Approximation bis zur zweiten Ordnung der Varianz vgl. auch Bakshi und Madan (2006). 
Für die Varianz:

$$
\begin{aligned}
& V A R^{P}(R)-V A R^{Q}(R) \\
& \approx \underbrace{\underbrace{\gamma S K E W^{Q}(R) V A R^{Q}(R)^{3 / 2}}_{\text {bis zur ersten Ordnung }}+\frac{\gamma^{2}}{2}\left(K U R T^{Q}(R)-3\right) V A R^{Q}(R)^{2}}_{\text {bis zur zweiten Ordnung }}
\end{aligned}
$$

Für die Schiefe:

$$
\begin{aligned}
& S K E W^{P}(R)-S K E W^{Q}(R) \\
\approx & \underbrace{\gamma\left(K U R T^{Q}(R)-3\right) V A R^{Q}(R)^{1 / 2}}_{\text {bis zur ersten Ordnung }} \\
& \underbrace{+\frac{\gamma^{2}}{2}\left(P K E W^{Q}(R)-10 \cdot S K E W^{Q}(R)\right) V A R^{Q}(R)}_{\text {bis zur zweiten Ordnung }}
\end{aligned}
$$

Für die Kurtosis:

$$
\begin{aligned}
& K U R T^{P}(R)-K U R T^{Q}(R) \\
\approx & \underbrace{\gamma\left(P K E W^{Q}(R)-4 \cdot S K E W^{Q}(R)\right) V A R^{Q}(R)^{1 / 2}}_{\text {bis zur ersten Ordnung }} \\
& \underbrace{+\frac{\gamma^{2}}{2}\left(H E X I C^{Q}(R)-9 \cdot K U R T^{Q}(R)-4 \cdot S K E W^{Q}(R)^{2}+12\right) V A R^{Q}(R)}_{\text {bis zur zweiten Ordnung }}
\end{aligned}
$$

Aus den hergeleiteten Beziehungen lassen sich die Beobachtungen aus Abbildung 4.3 unmittelbar ableiten. So ist beispielhaft aus der Approximation für die Differenz in der Schiefe abzulesen, dass diese proportional zum Produkt aus Excess Kurtosis und Volatilität ist. Damit folgt, dass in unruhigen Phasen, in denen hohe Unsicherheit herrscht, ausgedrückt durch eine hohe implizite Volatilität und Kurtosis, die Differenz zwischen physischem und risikoneutralem Moment steigt. Für ruhige Marktphasen hingegen sinkt dieser Spread aufgrund der abnehmenden impliziten Unsicherheit.

Um zu beurteilen, in welchem Maße die exakte Differenz aus risikoadjustiertem und implizitem Moment durch die hergeleiteten Approximationen reproduziert werden kann, wird folgendes Regressionsmodell geschätzt:

$$
\text { Differenz }=\alpha+\beta \cdot \text { Approximation. }
$$


Tabelle 4.4 zeigt die adjustierten Bestimmtheitsmaße aus den Regressionen, jeweils für die Approximation bis zur ersten und bis zur zweiten Ordnung.

Tabelle 4.4: Bestimmtheitsmaße für die Erklärung der Differenz aus risikoadjustiertem und implizitem Moment durch die Approximation bis zur ersten und zweiten Ordnung

\begin{tabular}{ccccccc}
\hline & \multicolumn{3}{c}{ bis zur ersten Ordnung } & \multicolumn{2}{c}{ bis zur zweiten Ordnung } \\
\hline & VAR & SKEW & KURT & VAR & SKEW & KURT \\
\hline 2 & $99,88 \%$ & $16,91 \%^{\dagger}$ & $79,41 \%^{\dagger}$ & $99,91 \%$ & $18,00 \%^{\dagger}$ & $79,23 \%^{\dagger}$ \\
4 & $96,90 \%$ & $4,28 \%$ & $15,72 \%^{\dagger}$ & $97,47 \%$ & $8,66 \%$ & $18,06 \%^{\dagger}$ \\
6 & $73,95 \%$ & $28,68 \%$ & $1,61 \%$ & $76,97 \%$ & $42,54 \%$ & $-0,33 \%$ \\
8 & $1,29 \%$ & $49,24 \%$ & $19,26 \%$ & $4,70 \%$ & $53,83 \%$ & $6,12 \%$ \\
10 & $29,67 \%^{\dagger}$ & $65,16 \%$ & $41,40 \%$ & $5,64 \%$ & $42,11 \%$ & $11,33 \%$ \\
12 & $52,45 \%^{\dagger}$ & $75,26 \%$ & $64,78 \%$ & $47,87 \%$ & $19,21 \%$ & $10,52 \%$ \\
\hline
\end{tabular}

Diese Tabelle zeigt die adjustierten Bestimmtheitsmaße des Regressionsmodells Differenz $=\alpha+\beta$. Approximation für die Differenz aus risikoadjustierten (CRRA Nutzenfunktionen) und impliziten Momenten und der Approximation durch die risikoneutralen Momente bis zur ersten und zweiten Ordnung. $\dagger$ gibt an, ob der geschätzte Slope-Koeffizient negativ ist. Die Werte beziehen sich auf Daten von Optionen auf den S\&P 500 mit einer Laufzeit von einem Monat. Der Zeitraum beträgt Januar 1996 bis Oktober 2010.

Für die Varianz kann die Approximation bis zur ersten Ordnung die Dynamik des exakten Spreads für weniger risikoaverse Investoren (RRA 2, 4 und 6) zufriedenstellend reproduzieren. Die Güte wird bei Verwendung der Approximation bis zur zweiten Ordnung leicht erhöht. Für risikoaversere Investoren (RRA 8, 10 und 12) hingegen sind die Regressionsergebnisse im Fall der Approximation bis zur ersten Ordnung nicht akzeptal. Das adjustierte Bestimmtheitsmaß ist niedriger als 2\% (RRA 8) oder die Koeffizienten des Slope Koeffizienten sind negativ (RRA 10 und 12). Durch die genauere Approximation zeigen die Ergebnisse für die Aversionen 8 und 10 erneut Bestimmtheitsmaße unter $6 \%$. Für die Aversion 12 jedoch ist das Ergebnis zufriedenstellend und zeigt, dass die genauere Approximation der Approximation bis zur ersten Ordnung vorzuziehen ist, insbesondere werden für alle betrachteten Fälle positive Slope-Koeffizienten geschätzt.

Für Schiefe und Kurtosis zeigen die Regressionsmodelle im Gegensatz zur Varianz, dass die Approximation bis zur ersten Ordnung die Dynamik des exakten Spreads für risikoaversere Investoren (RRA 8, 10 und 12) zufriedenstellend reproduzieren kann. Die Güte der Prognose wird bei Verwendung der genaueren Approximation jedoch verringert. Dieses Ergebnis könnte darauf zurückzuführen sein, dass diese Approximationen fünfte und sechste Momente verwenden und dass diese Größen numerisch schwer unverzerrt bestimmbar sind. Für weniger risikoaverse Investoren hingegen (RRA 2 und 4) liefert das Regressionsmodell für die Approximation bis zur ersten und zweiten Ordnung geringe Bestimmtheitsmaße oder sogar negative Slope-Koeffizienten. Im Vergleich der Prognosegüten für die Approximation bis zur ersten und zweiten Ordnung liefert die genauere 
Approximation keine unmittelbaren Vorteile gegenüber der Approximation bis zur ersten Ordnung.

Es lässt sich festhalten, dass die Approximationen die exakte Differenz in den Tendenzen abbildet, die Prognosegüten für unterschiedliche Risikoaversionen jedoch stark varriieren. Die Dynamik kann durch die Approximation bis zur ersten Ordnung für die Varianz für weniger risikoaverse Investoren, für Schiefe und Kurtosis hingegen für stark risikoaverse Investoren zufriedenstellend reproduziert werden. Die Verwendung der exakteren Approximation ist für die Varianz im Vergleich zu der Approximation bis zur ersten Ordnung vorzuziehen, für Schiefe und Kurtosis zeigt sich keine Verbesserung.

Im Fall von zeitvariablen Risikoaversionen haben die Ergebnisse zur Konsequenz, dass in ruhigen Marktphasen, in denen eher geringe Risikoaversionen zu vermuten sind, die Dynamik des Spreads für die Varianz durch die Approximation bis zur ersten Ordnung nachgebildet werden kann, während in Marktturbulenzen, in welchen hohe Risikoaffinität herrscht, ausschließlich für Schiefe und Kurtosis. In diesem Fall kann die Prognose für die Varianz durch die Verwendung der Approximation bis zur zweiten Ordnung deutlich verbessert werden. Dieses Ergebnis steht auch im Einklang mit den Erkenntnissen aus Abbildung 4.3, in welcher deutlich wurde, dass gerade in unruhigen Marktphasen die Spreads zwischen den Risikoaversionen stark divergieren. Insbesondere die Hinzunahme der Kurtosis in die Approximation, welche als Indikator für extreme Kursschwankungen gesehen werden kann, hat somit einen positiven Einfluss auf die Prognosegüte.

\subsection{Fähigkeit zur Prognose realisierter Momente}

Der folgende Abschnitt untersucht die Prognosefähigkeit der risikoadjustierten modellfreien impliziten Momente für die entsprechenden realisierten Größen. Die Beurteilung der Prognosegüte erfolgt auf Basis des Regressionsmodells

$$
\text { Realized }=\alpha+\beta \cdot \text { Predicted }
$$

Im Weiteren erfolgt die Überprüfung der Signifikanz der Koeffizienten $\alpha$ und $\beta$ durch Wald-Tests auf die Hypothesen $\alpha=0, \beta=1$ sowie auf die gemeinsame Hypothese $\alpha=0$ und $\beta=1$. Die Momente, welche bzgl. der Risikoaversion 0 bestimmt werden, entsprechen den impliziten Momenten aus Bakshi, Kapadia und Madan (2003) und dienen für die Untersuchung als wichtiger Benchmark gegenüber den risikoadjustierten. 


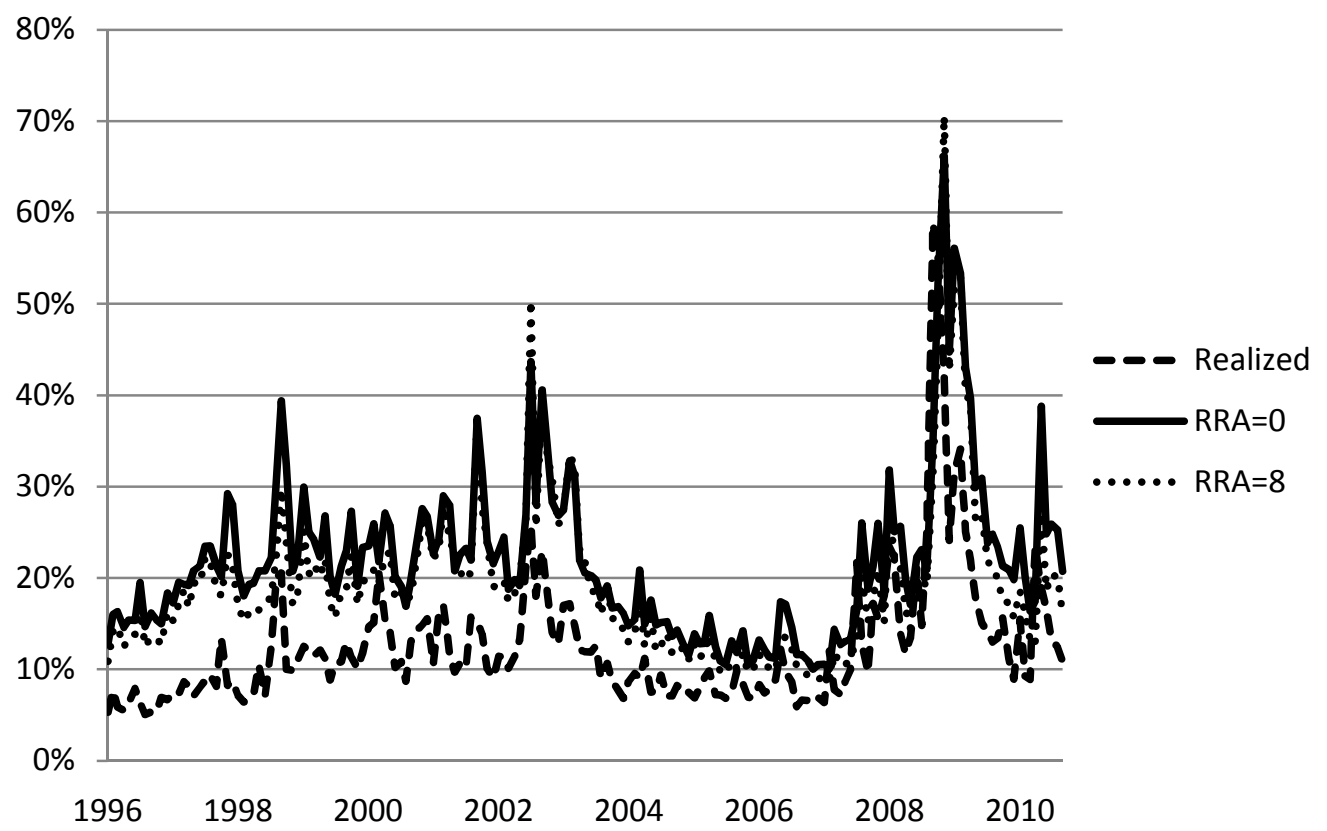

Abbildung 4.4: Diese Abbildung zeigt die realisierte, die implizite $(R R A=0)$ und die risikoadjustierte (CRRA Nutzenfunktion, RRA=8) Volatilität. Alle Werte sind annualisiert. Die Werte beziehen sich auf Daten von Optionen auf den S\&P 500 mit einer Laufzeit von einem Monat. Der Zeitraum beträgt Januar 1996 bis Oktober 2010.

\subsubsection{Ergebnisse für die Volatilität}

Tabelle 4.5: Deskriptive Statistiken und Ergebnisse des Regressionsmodells für die realisierten, impliziten und risikoadjustierten Volatilitäten

\begin{tabular}{ccccccc}
\hline & ERW & STD & MAE & Intercept & Slope & Adj. $R^{2}$ \\
\hline 0 & 0,219 & 0,089 & 0,099 & $-0,013$ & $0,631^{* * *}$ & $58,15 \%$ \\
2 & 0,202 & 0,075 & 0,084 & $-0,026^{* *}$ & $0,746^{* * *}$ & $56,83 \%$ \\
4 & 0,192 & 0,071 & 0,076 & $-0,026^{* *}$ & $0,782^{* * *}$ & $56,30 \%$ \\
6 & 0,190 & 0,077 & 0,074 & $-0,012$ & $0,718^{* * *}$ & $56,37 \%$ \\
8 & 0,194 & 0,090 & 0,077 & 0,005 & $0,619^{* * *}$ & $56,04 \%$ \\
10 & 0,202 & 0,105 & 0,085 & $0,018^{* *}$ & $0,527^{* * *}$ & $55,39 \%$ \\
12 & 0,214 & 0,120 & 0,095 & $0,027^{* * *}$ & $0,454^{* * *}$ & $54,70 \%$ \\
Realized & 0,125 & 0,074 & & & & \\
\hline
\end{tabular}

Diese Tabelle zeigt deskriptive Statistiken und Ergebnisse des Regressionsmodells Realized $=\alpha+\beta$. Predicted für die realisierte (Realized) und die implizite (RRA 0) Volatilität sowie die risikoadjustierten Volatilitäten (CRRA Nutzenfunktionen). Alle Werte sind annualisiert. MAE gibt den mittleren absoluten Fehler zwischen dem entsprechenden und dem realisierten Wert an. Intercept und Slope entsprechen den geschätzten Werten von $\alpha$ und $\beta$. Adj. $R^{2}$ zeigt das adjustierte Bestimmtheitsmaß des Modells. Die Signifikanz der Koeffizienten, abgeleitet aus dem Wald-Test, wird auf dem 10\%-Niveau mit $*$, auf dem $5 \%$-Niveau mit $* *$ und auf dem 1\%-Niveau mit $* * *$ angegeben. Die Werte beziehen sich auf Daten von Optionen auf den S\&P 500 mit einer Laufzeit von einem Monat. Der Zeitraum beträgt Januar 1996 bis Oktober 2010. 
Abbildung 4.4 und Tabelle 4.5 zeigen die empirischen Ergebnisse für die Volatilität. Es lässt sich ein deutlicher Unterschied zwischen der realisierten und der rein impliziten $(\mathrm{RRA}=0)$ Volatilität erkennen. Dies kommt insbesondere auch in den Mittelwerten 21,9 $\%$ p.a. und 12,5 \% p.a. zum Ausdruck. Genauer liefert ein t-Test eine auf einem 1\%-Niveau statistisch signifikante positive Varianz-Risikoprämie.

Für die risikoadjustierten Momente sinkt die durchschnittliche Volatilität ausgehend von der rein impliziten $(\mathrm{RRA}=0)$ zunächst, bevor sie ab der Risikoaversion 6 wieder ansteigt. Dies gilt auch für die Standardabweichungen der Volatilitäten und insbesondere für den mittleren absoluten Fehler. Das Minimum im Sinne des mittleren absoluten Fehlers wird zwischen den Risikoaversionen 4 und 6 erreicht. Dies hat die Interpretation, dass für eine Risikoaversion des repräsentativen Investors aus diesem Intervall die Varianz-Risikoprämie am stärksten reduziert wird. Im weiteren Sinne bedeutet dies, dass der Übergang von der risikoneutralen zu der physischen Dichte für diese Risikoaversion die deutlichste Auswirkung hat. Die Ergebnisse des Regressionsmodells bestätigen die dargelegte Beobachtung. Für den Fall des Risikoaversionsparameters 4 wird der Slope-Koeffizient durch das Modell am nächsten bei eins geschätzt, ist davon jedoch statistisch signifikant verschieden. Dieses Resultat ist im Einklang mit der bestehenden Literatur. Bei Unterstellung einer CRRA Nutzenfunktion des repräsentativen Investors für den S\&P 500 schätzen Duan und Zhang (2010) einen Risikoaversionsparameter in Höhe von 4,25, Bliss und Panigirtzoglou (2004) wiederum einen Parameter von 4,08.

Das adjustierte Bestimmtheitsmaß wird nicht von der Risikoadjustierung verbessert, sondern wird gegenteilig leicht reduziert. Dennoch wird der sehr gute Erklärungsgehalt der impliziten Verfahren zur Beschreibung der zeitlichen Struktur der realisierten Volatilität (mit adjustierten Bestimmtheitsmaßen um 55\% - 60\%) bestätigt. ${ }^{31}$ 


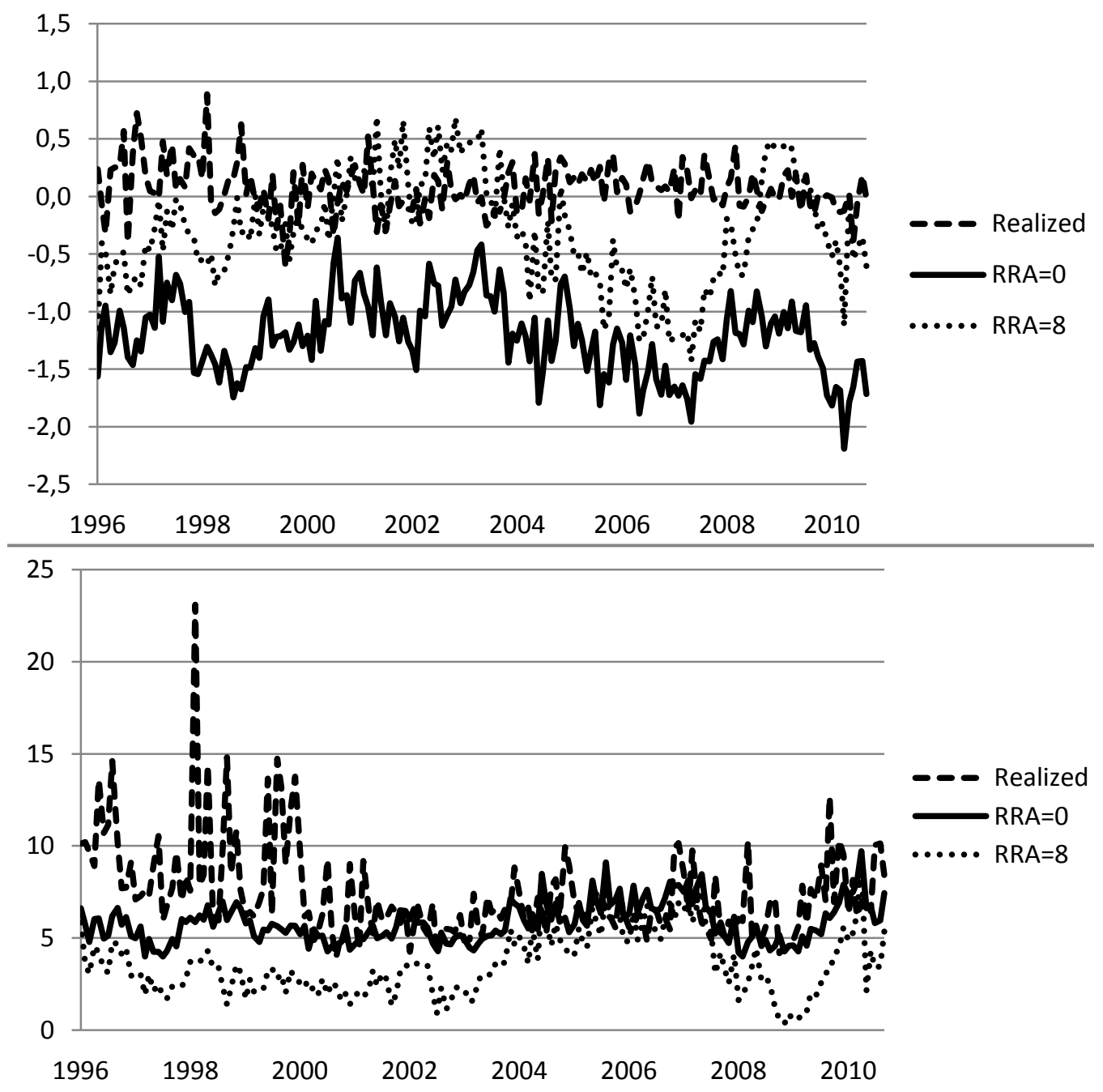

Abbildung 4.5: Diese Abbildung zeigt die realisierte, die implizite ( $R A A=0)$ und die risikoadjustierte (CRRA Nutzenfunktion, RRA=8) Schiefe und Kurtosis. Schiefe und Kurtosis sind standardisiert dargestellt. Die Werte beziehen sich auf Daten von Optionen auf den S\&P 500 mit einer Laufzeit von einem Monat. Der Zeitraum beträgt Januar 1996 bis Oktober 2010. 


\subsubsection{Ergebnisse für Schiefe und Kurtosis}

Tabelle 4.6: Deskriptive Statistiken und Ergebnisse des Regressionsmodells für die realisierten, impliziten und risikoadjustierten Schiefen und Kurtosin

\begin{tabular}{ccccccc}
\hline & ERW & STD & MAE & Intercept & Slope & Adj. $R^{2}$ \\
\hline Schiefe & & & & & & \\
\hline 0 & $-1,217$ & 0,334 & 1,287 & 0,029 & $-0,034^{* * *}$ & $-0,29 \%$ \\
2 & $-1,226$ & 0,384 & 1,296 & 0,011 & $-0,048^{* * *}$ & $0,17 \%$ \\
4 & $-0,979$ & 0,435 & 1,055 & 0,022 & $-0,049^{* * *}$ & $0,41 \%$ \\
6 & $-0,642$ & 0,482 & 0,763 & 0,041 & $-0,045^{* * *}$ & $0,45 \%$ \\
8 & $-0,309$ & 0,483 & 0,552 & $0,055^{* * *}$ & $-0,049^{* * *}$ & $0,64 \%$ \\
10 & $-0,032$ & 0,443 & 0,424 & $0,068^{* * *}$ & $-0,061^{* * *}$ & $1,00 \%$ \\
12 & 0,174 & 0,379 & 0,390 & $0,084^{* * *}$ & $-0,080^{* * *}$ & $1,42 \%$ \\
Realized & 0,070 & 0,215 & & & & \\
\hline Kurtosis & & & & & & \\
\hline 0 & 5,829 & 1,103 & 2,119 & $6,273^{* * *}$ & $0,193^{* * *}$ & $0,12 \%$ \\
2 & 6,229 & 1,287 & 2,041 & $6,367^{* * *}$ & $0,165^{* * *}$ & $0,13 \%$ \\
4 & 5,721 & 1,531 & 2,338 & $6,298^{* * *}$ & $0,192^{* * *}$ & $0,76 \%$ \\
6 & 4,692 & 1,707 & 3,090 & $6,737^{* * *}$ & $0,141^{* * *}$ & $0,31 \%$ \\
8 & 3,565 & 1,694 & 3,980 & $7,138^{* * *}$ & $0,073^{* * *}$ & $-0,34 \%$ \\
10 & 2,610 & 1,507 & 4,803 & $7,366^{* * *}$ & $0,012^{* * *}$ & $-0,57 \%$ \\
12 & 1,902 & 1,232 & 5,494 & $7,477^{* * *}$ & $-0,042^{* * *}$ & $-0,53 \%$ \\
Realized & 7,397 & 2,557 & & & & \\
\hline
\end{tabular}

Diese Tabelle zeigt deskriptive Statistiken und Ergebnisse des Regressionsmodells Realized $=\alpha+\beta$. Predicted für die realisierte (Realized), die implizite (RRA 0) und die risikoadjustierten standardisierten Schiefen und Kurtosin (CRRA Nutzenfunktionen). MAE gibt den mittleren absoluten Fehler zwischen dem entsprechenden und dem realsiserten Wert an. Intercept und Slope entsprechen den geschätzten Werten von $\alpha$ und $\beta$. Adj. $R^{2}$ zeigt das adjustierte Bestimmtheitsmaß des Modells. Die Signifikanz der Koeffizienten, abgeleitet aus dem Wald-Test, wird auf dem 10\%-Niveau mit $*$, auf dem $5 \%$-Niveau mit $* *$ und auf dem 1\%-Niveau mit $* * *$ angegeben. Die Werte beziehen sich auf Daten von Optionen auf den S\&P 500 mit einer Laufzeit von einem Monat. Der Zeitraum beträgt Januar 1996 bis Oktober 2010.

Abbildung 4.5 und Tabelle 4.6 stellen die Ergebnisse der Momentenprognose für die standardisierte Schiefe und Kurtosis dar. Im Gegensatz zu der Volatilität hat die Risikoadjustierung, wie im letzten Abschnitt beobachtet, einen deutlichen Einfluss auf die resultierenden Momente. Mit zunehmendem Risikoaversionsparameter steigt die mittlere Schiefe an und ist für den Risikoaversionsparameter 12 sogar positiv. Für die Kurtosis hingegen wird eine Verschiebung nach unten bzw. eine Verringerung erzielt. Für steigende Risikoaversionsparameter sinkt der mittlere absolute Fehler für die Schiefe, während er für die Kurtosis erhöht wird. Die Verschiebung bewirkt somit eine Reduktion bzw. Vergrößerung der Abstände zu den realisierten Momenten.

$31 \quad$ Vgl. Jiang und Tian (2005). 
Zur weiteren Beurteilung der Güte der Prognosefähigkeit für die realisierte Schiefe und Kurtosis werden die Ergebnisse der Regressionsmodelle betrachtet. Sowohl für Schiefe als auch Kurtosis sind die Resultate für die "rein" impliziten sowie für die risikoadjustierten Momente nicht zufriedenstellend. Es werden adjustierte Bestimmtheitsmaße unter $2 \%$ und zum Teil sogar negative Slope-Koeffizienten geschätzt, insbesondere für die Schiefe sind ausschließlich negative Koeffizienten ermittelt worden. Es lässt sich festhalten, dass durch die betrachteten impliziten Methoden sich der mittlere absolute Fehler, durch die Risikoadjustierung (insbesondere für die Schiefe) reduzieren lässt, die zeitliche Struktur jedoch nicht wiedergegeben werden kann. Insgesamt ist die Prognosefähigkeit für die realisierte Schiefe und Kurtosis nicht zufriedenstellend.

\subsubsection{Ergebnisse für Subperioden}

Zur Überprüfung der Robustheit der Ergebnisse wird der out-of-sample Zeitraum in zwei Subperioden unterteilt. Tabelle 4.7 zeigt die entsprechenden Resultate. 
Tabelle 4.7: Ergebnisse des Regressionsmodells für die realisierten, impliziten und risikoadjustierten höheren Momente für zwei Subperioden

\begin{tabular}{|c|c|c|c|c|c|c|}
\hline & Intercept & Slope & $\operatorname{Adj} . R^{2}$ & Intercept & Slope & $\operatorname{Adj} . R^{2}$ \\
\hline Volatilität & \multicolumn{3}{|c|}{$1996-2003$} & \multicolumn{3}{|c|}{$2003-2010$} \\
\hline 0 & $-0,017$ & $0,568^{* * *}$ & $60,95 \%$ & $-0,008$ & $0,695^{* * *}$ & $65,20 \%$ \\
\hline 2 & $-0,031^{* *}$ & $0,674^{* * *}$ & $63,72 \%$ & $-0,024^{*}$ & $0,845^{* *}$ & $65,11 \%$ \\
\hline 4 & $-0,032^{* * *}$ & $0,712^{* * *}$ & $64,89 \%$ & $-0,024^{*}$ & 0,893 & $64,83 \%$ \\
\hline 6 & $-0,018$ & $0,648^{* * *}$ & $64,25 \%$ & $-0,008$ & $0,812^{* * *}$ & $64,08 \%$ \\
\hline 8 & 0,000 & $0,547^{* * *}$ & $63,03 \%$ & 0,010 & $0,697^{* * *}$ & $63,08 \%$ \\
\hline 10 & 0,014 & $0,458^{* * *}$ & $62,04 \%$ & $0,025^{* *}$ & $0,593^{* * *}$ & $62,03 \%$ \\
\hline 12 & $0,024^{* * *}$ & $0,391^{* * *}$ & $61,33 \%$ & $0,035^{* * *}$ & $0,513^{* * *}$ & $61,16 \%$ \\
\hline Schiefe & \multicolumn{3}{|c|}{$1996-2003$} & \multicolumn{3}{|c|}{$2003-2010$} \\
\hline 0 & $-0,095$ & $-0,160^{* * *}$ & $2,79 \%$ & 0,130 & $0,054^{* * *}$ & $-0,14 \%$ \\
\hline 2 & $-0,098$ & $-0,163^{* * *}$ & $4,85 \%$ & $0,129^{*}$ & $0,052^{* * *}$ & $0,01 \%$ \\
\hline 4 & $-0,041$ & $-0,145^{* * *}$ & $4,62 \%$ & 0,082 & $0,021^{* * *}$ & $-0,89 \%$ \\
\hline 6 & 0,021 & $-0,125^{* * *}$ & $3,47 \%$ & 0,055 & $-0,005^{* * *}$ & $-1,14 \%$ \\
\hline 8 & $0,064^{* *}$ & $-0,123^{* * *}$ & $3,04 \%$ & $0,048^{*}$ & $-0,022^{* * *}$ & $-0,75 \%$ \\
\hline 10 & $0,099^{* * *}$ & $-0,147^{* * *}$ & $3,49 \%$ & $0,052^{* * *}$ & $-0,036^{* * *}$ & $-0,19 \%$ \\
\hline 12 & $0,142^{* * *}$ & $-0,199^{* * *}$ & $4,53 \%$ & $0,061^{* * *}$ & $-0,048^{* * *}$ & $0,20 \%$ \\
\hline Kurtosis & \multicolumn{3}{|c|}{$1996-2003$} & \multicolumn{3}{|c|}{$2003-2010$} \\
\hline 0 & 1,547 & 1,184 & $6,43 \%$ & $5,548^{* * *}$ & $0,210^{* * *}$ & $1,40 \%$ \\
\hline 2 & 3,035 & 0,855 & $6,01 \%$ & $5,842^{* * *}$ & $0,152^{* * *}$ & $0,46 \%$ \\
\hline 4 & $2,767^{*}$ & 1,007 & $9,87 \%$ & $5,837^{* * *}$ & $0,162^{* * *}$ & $1,79 \%$ \\
\hline 6 & $3,241^{* *}$ & 1,183 & $12,90 \%$ & $6,224^{* * *}$ & $0,118^{* * *}$ & $0,86 \%$ \\
\hline 8 & $4,600^{* * *}$ & 1,206 & $11,42 \%$ & $6,541^{* * *}$ & $0,074^{* * *}$ & $-0,41 \%$ \\
\hline 10 & $5,647^{* * *}$ & 1,235 & $8,07 \%$ & $6,689^{* * *}$ & $0,052^{* * *}$ & $-0,87 \%$ \\
\hline 12 & $6,316^{* * *}$ & 1,277 & $4,55 \%$ & $6,694^{* * *}$ & $0,067^{* * *}$ & $-0,84 \%$ \\
\hline
\end{tabular}

Diese Tabelle zeigt die Ergebnisse des Regressionsmodells Realized $=\alpha+\beta \cdot$ Predicted für die realisierten (Realized), die impliziten (RRA 0) und risikoadjustierten höheren Momente (CRRA Nutzenfunktionen) für zwei Subperioden. Intercept und Slope entsprechen den geschätzten Werten von $\alpha$ und $\beta$. Adj. $R^{2}$ zeigt das adjustierte Bestimmtheitsmaß des Modells. Die Signifikanz der Koeffizienten, abgeleitet aus dem Wald-Test, wird auf dem 10\%-Niveau mit *, auf dem 5\%-Niveau mit ** und auf dem $1 \%$-Niveau mit $* * *$ angegeben. Die Werte beziehen sich auf Daten von Optionen auf den S\&P 500 mit einer Laufzeit von einem Monat. Die erste Subperiode umfasst den Zeitraum Januar 1996 bis Mai 2003. Der Zeitraum der zweiten Subperiode beträgt Juni 2003 bis Oktober 2010.

Die wesentlichen, bisher gewonnen Erkenntnisse werden bestätigt. In beiden Subperioden wird die gute Prognosefähigkeit der realisierten Volatilität verifiziert. Die SlopeKoeffizienten sind wiederum für den Risikoaversionsparameter 4 in beiden Perioden am nächsten an eins. In der zweiten Subperiode ist dieser Wert sogar nicht statistisch signifikant von eins verschieden. Dies bedeutet, dass der Intercept-Parameter in diesem Fall als Reduktion der Varianz-Risikoprämie interpretiert werden kann. Das adjustierte Bestimmtheitsmaß bleibt durch die Risikoadjustierung in beiden Subperioden gegenüber der impliziten Volatilität fast unverändert. 
In beiden Subperioden zeigen die Regressionen für die Schiefe eine schlechte Erklärung der zeitlichen Struktur durch die impliziten Verfahren. In der ersten Subperiode sind die adjustierten Bestimmtheitsmaße zwar geringfügig erhöht, jedoch werden in allen betrachteten Fällen negative Slope-Koeffizienten geschätzt.

Für die Kurtosis wiederum liefern die Ergebnisse der Regressionsmodelle gegensätzliche Resultate und müssen differenziert betrachtet werden. In der ersten Subperiode werden in allen Fällen für den Slope-Koeffizienten Werte um eins geschätzt. Der Wald-Test kann sogar die Hypothese, dass der Koeffizient eins ist, auf einem 10\%-Niveau für kein Modell verwerfen. Für die "rein" implizite Kurtosis und die risikoadjustierte Kurtosis mit der Risikoaversion 2 ist in diesem Fall der Intercept-Koeffizient sogar nicht statistisch verschieden von null. Die gemeinsame Hypothese $\alpha=0$ und $\beta=1$ wird jedoch auf einem 1\%-Niveau verworfen. Ab der Risikoadjustierung mit einem Risikoaversionsparameter größer als 2 wird die realisierte Kurtosis durch den impliziten und risikoadjustierten Moment, ausgedrückt durch den um eins geschätzten Intercept-Koeffizienten, systematisch unterschätzt. Auch die Erklärung der zeitlichen Struktur wird durch die Risikoadjustierung verbessert. Beispielsweise verdoppelt sich das adjustierte Bestimmtheitsmaß im Vergleich zwischen dem "rein" impliziten und dem mit der Risikoaversion 6 risikoadjustiertem Modell annähernd (6,43\% vs. 12,90\%). Insgesamt ist insbesondere im Vergleich zu dem vollständigen Zeitraum in dieser Subperiode die Güte der Prognose in allen Fällen deutlich erhöht. In der zweiten Subperiode indes sind die Ergebnisse, insbesondere im Hinblick auf das adjustierte Bestimmtheitsmaß, enttäuschend und erklären die aggregiert schlechte Prognose für den gesamten Zeitraum.

\subsubsection{Ergebnisse für zeitvariable Risikoaversionen}

In den bisherigen Untersuchungen ist die restriktive Annahme getroffen worden, dass die Risikoaversion des repräsentativen Investors im Zeitverlauf konstant ist. Der Übergang zu zeitvariablen Aversionen bedingt die Verwendung historischer Daten für die Parameterschätzung. Die Risikoaversion kann dabei auf verschiedene Weise gewonnen werden. Bliss und Panigirtzoglou (2004) nutzen hierfür die Berkowitz-Statistik (vgl. Kapitel 3). Duan und Zhang (2010) verwenden eine Approximation über den Volatility-Spread gemäß Bakshi und Madan (2006). Diese Werte liegen für den Zeitraum von Januar 2001 bis Oktober 2009 vor (vgl. Abbildung 4.6) und sollen im Folgenden für die Risikoadjustierung verwendet werden. Der mittlere Wert entspricht 4,253.

Zunächst sei jedoch ein weiterer historischer Ansatz ohne Verwendung von Optionsmarktdaten betrachtet. Zur Bestimmung der Risikoaversion wird die First-Order-Condition des repräsentativen CRRA Investors erneut bemüht. Hierzu sei das Polynom aus Satz 2 nicht 


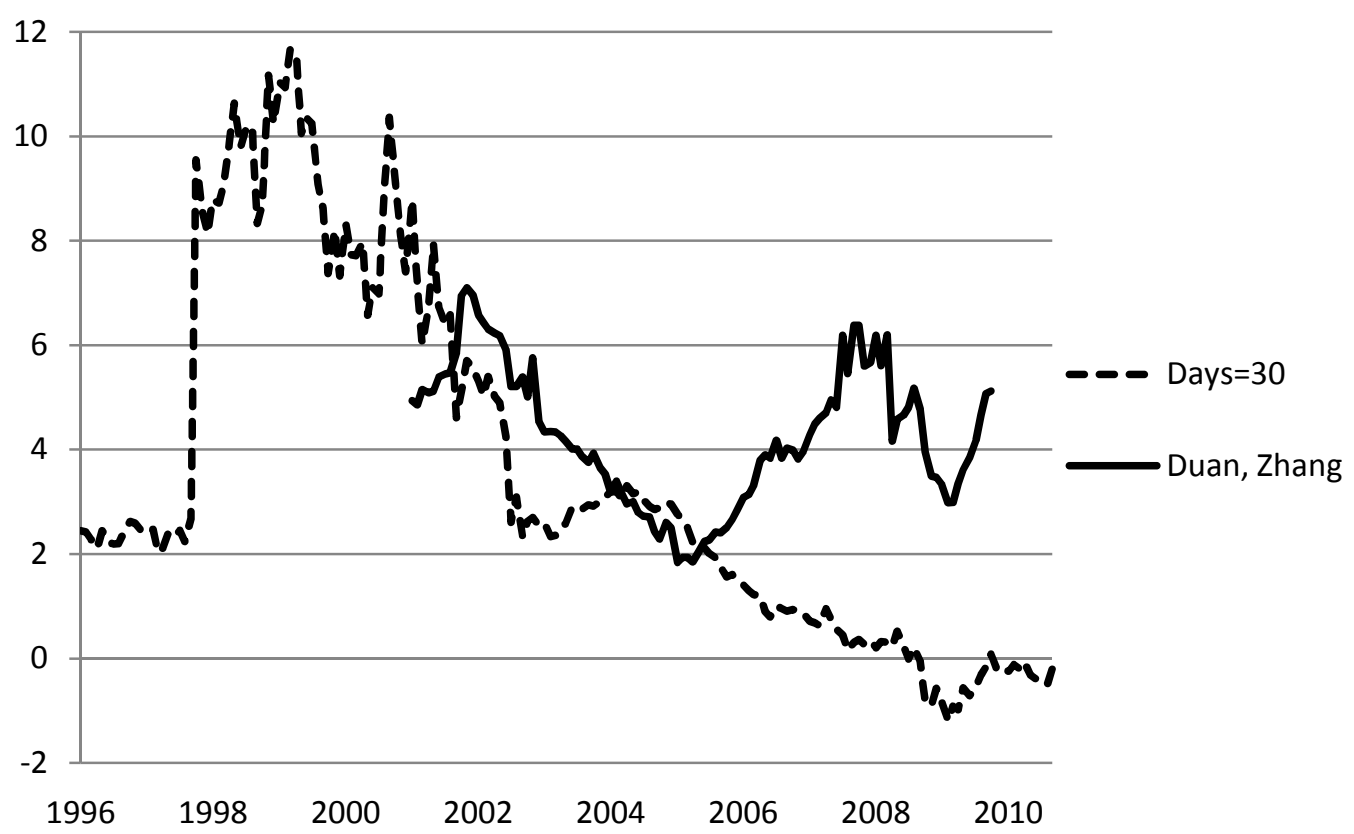

Abbildung 4.6: Diese Abbildung zeigt die geschätzten Risikoaversionen des S\&P 500 auf Basis von historischen Daten der letzten 120 Monate für einen Horizont von 30 Tagen sowie die geschätzten Risikoaversionen von Duan und Zhang (2010). Der Zeitraum beträgt Januar 1996 bis Oktober 2010 bzw. Januar 2001 bis Oktober 2009.

nach dem Erwartungswert, sondern nach der Risikoaversion aufgelöst. Konkret werden die höheren Momente sowie der Erwartungswert aus den letzten 120 Monaten geschätzt und in die First-Order Condition eingesetzt. Eine Zielwertsuche liefert die gewünschte Risikoaversion. Abbildung 4.6 zeigt die ermittelten Risikoaversionen im Zeitverlauf. Der mittlere Wert der Zeitreihe ist 3,566. Dieser Wert ist vergleichbar mit dem Wert aus Bliss und Panigirtzoglou (2004) von 4,08. 
Tabelle 4.8: Deskriptive Statistiken für die Excess Returns und die risikoadjustierten höheren Momenten für geschätzte Risikoaversionsparameter und Ergebnisse des Regressionsmodells

\begin{tabular}{lcccccc}
\hline & ERW & STD & MAE & Intercept & Slope & Adj. $R^{2}$ \\
\hline Excess Return & 0,088 & 0,168 & & & & \\
Volatilität & 0,241 & 0,252 & 0,133 & $0,087^{* * *}$ & $0,154^{* * *}$ & $27,44 \%$ \\
Schiefe & $-1,062$ & 1,460 & 1,191 & $0,055^{* * *}$ & $-0,014^{* * *}$ & $0,32 \%$ \\
Kurtosis & 11,210 & 32,970 & 10,111 & $7,453^{* * *}$ & $-0,005^{* * *}$ & $-0,14 \%$ \\
\hline
\end{tabular}

Diese Tabelle zeigt für geschätzte Risikoaversionsparameter für einen Investor mit CRRA Nutzenfunktion auf Basis von 120 Monaten historischer Beobachtungen für einen Horizont von 30 Tagen deskriptive Statistiken für die Excess Returns und die risikoadjustierten höheren Momente. Volatilitäten sind annualisert, Schiefen und Kurtosin standardisiert. MAE gibt den mittleren absoluten Fehler zwischen dem entsprechenden und dem realisierten Wert an. Des Weiteren zeigt die Tabelle die Ergebnisse des Regressionsmodells Realized $=\alpha+\beta \cdot$ Predicted. Intercept und Slope entsprechen den geschätzten Werten von $\alpha$ und $\beta$. Adj. $R^{2}$ zeigt das adjustierte Bestimmtheitsmaß des Modells. Die Signifikanz der Koeffizienten, abgeleitet aus dem Wald-Test, wird auf dem 10\%-Niveau mit $*$, auf dem 5\%-Niveau mit $* *$ und auf dem $1 \%$-Niveau mit $* * *$ angegeben. Die Werte beziehen sich auf Daten von Optionen auf den S\&P 500 mit einer Laufzeit von einem Monat. Der Zeitraum beträgt Januar 1996 bis Oktober 2010.

Tabelle 4.8 zeigt die Ergebnisse bei Verwendung der zeitvariablen Risikoaversionen. Die Resultate sind im Vergleich zu den "rein" impliziten Momenten und insbesondere zu der Risikoadjustierung mit dem Parameter 4 enttäuschend. Das Regressionsmodell liefert zwar für die Volatilität ein adjustiertes Bestimmtheitsmaß von 27,44\%, der Slope-Parameter wird jedoch deutlich geringer als eins geschätzt. Auch für die Schiefe und Kurtosis liefert der Übergang zu zeitvariablen Risikoaversionen in diesem Fall keine Verbesserung gegenüber dem statischen Modell. 
Tabelle 4.9: Deskriptive Statistiken für die risikoadjustierten Momente für die zeitvariablen Risikoaversionen aus Duan und Zhang (2010) und Ergebnisse des Regressionsmodells

\begin{tabular}{ccccccc}
\hline & ERW & STD & MAE & Intercept & Slope & Adj. $R^{2}$ \\
\hline Volatilität & & & & & & \\
\hline 0 & 0,219 & 0,108 & 0,089 & $-0,006$ & $0,651^{* * *}$ & $63,75 \%$ \\
2 & 0,202 & 0,091 & 0,076 & $-0,018$ & $0,762^{* * *}$ & $61,93 \%$ \\
4 & 0,194 & 0,087 & 0,069 & $-0,016$ & $0,786^{* * *}$ & $60,66 \%$ \\
6 & 0,194 & 0,096 & 0,069 & $-0,001$ & $0,711^{* * *}$ & $60,00 \%$ \\
8 & 0,200 & 0,111 & 0,075 & 0,015 & $0,608^{* * *}$ & $59,11 \%$ \\
Zeitvariabel & 0,194 & 0,087 & 0,070 & $-0,014$ & $0,776^{* * *}$ & $59,35 \%$ \\
\hline Schiefe & & & & & & \\
\hline 0 & $-1,188$ & 0,328 & 1,244 & $-0,008$ & $-0,054^{* * *}$ & $0,13 \%$ \\
2 & $-1,173$ & 0,373 & 1,229 & $-0,010$ & $-0,057^{* * *}$ & $0,63 \%$ \\
4 & $-0,905$ & 0,464 & 0,971 & 0,013 & $-0,048^{* * *}$ & $0,74 \%$ \\
6 & $-0,573$ & 0,559 & 0,709 & 0,033 & $-0,040^{* * *}$ & $0,81 \%$ \\
8 & $-0,267$ & 0,577 & 0,584 & $0,045^{* *}$ & $-0,043^{* * *}$ & $1,15 \%$ \\
Zeitvariabel & $-0,829$ & 0,553 & 0,931 & 0,006 & $-0,061^{* * *}$ & $2,97 \%$ \\
\hline Kurtosis & & & & & & \\
\hline 0 & 5,887 & 1,140 & 1,331 & $4,942^{* * *}$ & $0,257^{* * *}$ & $3,02 \%$ \\
2 & 6,233 & 1,173 & 1,344 & $5,332^{* * *}$ & $0,180^{* * *}$ & $1,11 \%$ \\
4 & 5,701 & 1,521 & 1,599 & $5,371^{* * *}$ & $0,190^{* * *}$ & $2,91 \%$ \\
6 & 4,771 & 1,853 & 2,200 & $5,698^{* * *}$ & $0,159^{* * *}$ & $3,04 \%$ \\
8 & 3,793 & 1,869 & 2,863 & $5,890^{* * *}$ & $0,149^{* * *}$ & $2,63 \%$ \\
Zeitvariabel & 5,428 & 1,716 & 1,787 & $5,488^{* * *}$ & $0,178^{* * *}$ & $3,37 \%$ \\
\hline
\end{tabular}

Diese Tabelle zeigt für die zeitvariablen Risikoaversionsparameter aus Duan und Zhang (2010) sowie für konstante Werte (CRRA Nutzenfunktion) die deskriptive Statistik für die risikoadjustierten Momente. Die Volatilitäten sind annualisert, Schiefen und Kurtosin standardisiert. MAE gibt den mittleren absoluten Fehler zwischen dem entsprechenden und dem realisierten Wert an. Des Weiteren zeigt die Tabelle die Ergebnisse des Regressionsmodells Realized $=\alpha+\beta \cdot$ Predicted. Intercept und Slope entsprechen den geschätzten Werten von $\alpha$ und $\beta$. Adj. $R^{2}$ zeigt das adjustierte Bestimmtheitsmaß des Modells. Die Signifikanz der Koeffizienten, abgeleitet aus dem Wald-Test, wird auf dem 10\%-Niveau mit $*$, auf dem $5 \%$-Niveau mit $* *$ und auf dem $1 \%$-Niveau mit $* * *$ angegeben. Die Werte beziehen sich auf Daten von Optionen auf den S\&P 500 mit einer Laufzeit von einem Monat. Der Zeitraum beträgt Januar 2001 bis Oktober 2009.

Die Ergebnisse für die Risikoaversionen aus Duan und Zhang (2010) zeigt Tabelle 4.9. Zum Vergleich sind auch die Resultate für den statischen Fall mit konstanten Risikoaversionen ergänzt. Für die Volatilität liefern die zeitvariablen Aversionen sowohl im Sinne der deskriptiven Metriken als auch im Sinne des Regressionsmodells vergleichbare Ergebnisse wie die konstante Risikoaversion 4. Für Schiefe und Kurtosis sind die adjustierten Bestimmtheitsmaße gegenüber dem statischen Modell geringfügig erhöht, jedoch wird wiederum für die Schiefe ein negativer Slope-Koeffizient, für die Kurtosis Bestimmtheitsmaße unter 4\% geschätzt. Es lässt sich festhalten, dass durch die Verwendung zeitvariabler Aversionen 
im Vergleich zu dem statischen Modell keine wesentlichen Verbesserungen erzielt werden können.

\subsection{Diskussion}

In dieser Studie wurde eine analytische Lösung zur direkten Risikoadjustierung impliziter höherer Momente der Log-Renditeverteilung präsentiert. Das Resultat beruht ausschließlich auf der Existenz eines repräsentativen Investors mit bekannter Nutzenfunktion und europäischen OTM-Optionen. Die hergeleiteten Momente stellen eine direkte Erweiterung der Momente aus Bakshi, Kapadia und Madan (2003) dar.

In einer empirischen Studie wurde die Prognosefähigkeit der risikoadjustierten Momente auf Basis von repräsentativen Investoren mit CRRA Nutzenfunktionen und unterschiedlichen Risikoaversionen für realisierte Momente des S\&P 500 untersucht und insbesondere mit den nicht-risikoadjustierten bzw. risikoneutralen Momenten verglichen. Um vollständig auf historische Daten verzichten zu können, wurde die Annahme getroffen, dass der repräsentative Investor durch einen konstanten, nicht-zeitvariablen Risikoaversionsparameter beschrieben wird.

Die Ergebnisse zeigen, dass die realisierte Volatilität im Einklang mit der bestehenden Literatur durch die impliziten Verfahren zufriedenstellend prognostiziert werden kann. Die Erklärung der zeitlichen Struktur, ausgedrückt durch das adjustierte Bestimmtheitsmaß, konnte im Vergleich zu dem "rein" impliziten Fall nicht durch die Risikoadjustierung verbessert werden. Die adjustierten Momente liefern jedoch teilweise eine geringere Verzerrung und somit eine Reduktion der Varianz-Risikoprämie. Dies gelingt insbesondere für Risikoaversionen aus dem Intervall 4 bis 6 . Dieses Resultat ist konform mit der bestehenden Literatur, in welcher Risikoaversionen für den repräsentativen Investor des S\&P 500 in Höhe von 4,25 (vgl. Duan und Zhang (2010)) und 4,08 (vgl. Bliss und Panigirtzoglou (2004)) ermittelt wurden.

Neben der Volatilität wurde auch die Prognosefähigkeit für die höheren Momente Schiefe und Kurtosis untersucht. Die realisierten Momente werden jedoch weder durch die risikoneutralen noch durch die risikoadjustierten Momente zufriedenstellend prognostiziert. Zwar kann der absolute Fehler durch die Risikoadjustierung (insbesondere für die Schiefe) verbessert werden, die zeitliche Struktur hingegen wird durch die impliziten Verfahren nicht wiedergegeben.

In diesem Zusammenhang sollte auch die verwendete Methodik zur Bestimmung realisierter Momente kritisch hinterfragt werden. Inwieweit insbesondere Intraday-Daten auf minütlicher Basis realisierte Schiefen und Kurtosin auf Monatsbasis approximieren, ist ei- 
ne weiterführende Frage. ${ }^{32}$ Im Speziellen ist für eine monatliche Betrachtungsweise unklar, wie eine Skalierung von Intraday-Momenten vorgenommen werden kann. Ein vielversprechenderer Ansatz zur Lösung dieses Problems ist die Methodik zur Bestimmung der realisierten Schiefe von Neuberger (2012). Eine Anwendung dieser Methodik zeigt, durch den Vergleich von realisierter und impliziter Schiefe, die Existenz einer Schiefe-Risikoprämie (vgl. Kozhan, Neuberger und Schneider (2013)).

Die Überprüfung der Robustheit der Ergebnisse erfolgte anschließend, indem der untersuchte Zeitraum in zwei Subperioden unterteilt und die Prognosefähigkeit erneut überprüft wurde. Die wesentlichen Resultate konnten bestätigt werden. Lediglich in der ersten Subperiode zeigen die Regressionen für die Kurtosis eine verbesserte Güte der Prognose, jedoch verbleibt eine systematische Unterschätzung des realisierten Wertes. Die restriktive Annahme von konstanten Risikoaversionsparametern des repräsentativen Investors wurde aufgegeben, indem auch zeitvariable Aversionen getestet wurden. Zum einen wurden diese Aversionen aus historischen Renditezeitreihen der letzten 120 Monate bestimmt, welches zu einer Verschlechterung der Prognosefähigkeit führt. Zum anderen wurden die aus Duan und Zhang (2010) bereitgestellten Aversionen, welche aus dem Volatilitäts-Spread gemäß Bakshi und Madan (2006) bestimmt wurden, getestet. Die Ergebnisse zeigen keine wesentliche Verbesserung der erzielten Resultate. Die erhaltenen Werte sind vergleichbar zu dem Fall, dass die Risikoaversion konstant gleich 4 ist. Es lässt sich festhalten, dass der Übergang zu zeitvariablen Aversionen im Vergleich zu dem statischen Fall keine deutliche Verbesserung bewirkt.

Zusammenfassend lässt sich das Fazit ziehen, dass die Volatilität im Einklang mit der bestehenden Literatur durch implizite Verfahren zufriedenstellend prognostiziert werden kann. Die Risikoadjustierung bewirkt eine Reduzierung der Varianz-Risikoprämie, jedoch keine Verbesserung der Prognose im Sinne des adjustierten Bestimmtheitsmaßes. Für Schiefe und Kurtosis hingegen zeigen die Ergebnisse sowohl für die risikoneutralen als auch für die risikoadjustierten Momente keine gute Prognosefähigkeit für die entsprechenden (aus Intraday-Daten bestimmten) realisierten Werte.

Ein weiterer Schwerpunkt wurde in der empirischen Studie auf die Wirkung der Risikoadjustierung auf die impliziten Momente gelegt. Hierzu wurde insbesondere die Differenz zwischen risikoadjustiertem und risikoneutralem Moment betrachtet.

Für die Volatilität wurde eine geringe negative Differenz zwischen den Momenten beobachtet. Im Speziellen liefert die Risikoadjustierung für unterschiedliche Risikoaversionen

32 Die Untersuchung für Momente geschätzt aus Tagesrenditen ergaben vergleichbare Resultate. 
vergleichbare Volatilitäten. Lediglich in Phasen hoher Marktunsicherheit divergieren die Momente.

Für die Schiefe und Kurtosis hingegen wird durch die Risikoadjustierung eine systematische Verschiebung bewirkt. Die Verschiebung folgt dabei den Präferenzen eines nichtgesättigten, risikoscheuen Investors. Für die Differenz der Schiefe zeigt sich bei Erhöhung des Risikoaversionsparameters ein steigender, für die Differenz der Kurtosis ein sinkender Trend. Diese Differenzen sind nicht konstant und zeitvariabel. In Phasen hoher Marktunsicherheit, welche insbesondere in der impliziten Volatilität zum Ausdruck kommt, steigt die Differenz, im Falle ruhiger Marktphasen verringert sich der Spread. Es lässt sich abschließend folgern, dass in Phasen hoher Marktunsicherheit der repräsentative Investor eine höhere Kompensation für die Übernahme von Risiken aus Schiefe und Kurtosis verlangt.

Um die Dynamik des Spreads genauer zu studieren, wurden Approximationen in Anlehnung an Bakshi, Kapadia und Madan (2003) über die risikoneutralen Momente und die Risikoaversion des repräsentativen Investors hergeleitet. Anhand dieser Approximationen lässt sich die Zeitvariabilität des Spreads ableiten. Eine Regressionsanalyse zeigt, dass die Dynamik für die Volatilität durch die Approximation bis zur zweiten Ordnung zum Teil akzeptabel reproduziert werden kann. Für Schiefe und Kurtosis sind die Ergebnisse lediglich für risikoaversere Investoren für die Approximation bis zur ersten Ordnung zufriedenstellend.

Im Rahmen der zentralen Forschungsfragen dieser Arbeit zur Asset Allokation mit höheren Momenten und impliziten Informationen sind zwei Erkenntnisse aus der empirischen Studie festzuhalten. Zum einen wird durch die Risikoadjustierung die Erklärung der zeitlichen Struktur realisierter Momente der Log-Renditeverteilung nicht verbessert, zum anderen wird lediglich die Verzerrung der Varianz und der Schiefe durch die Transformation (leicht) verringert.

In Bezug auf die Anwendung der risikoadjustierten Momente in der Asset Allokation muss beachtet werden, dass in der obigen Situation ein statisches Design mit konstanten, nicht zeitvariablen Aversionen gewählt wurde. Diese restriktive Annahme ist in Anwendungen jedoch nicht haltbar, vielmehr muss, wie beispielsweise in Duan und Zhang (2010) oder Kapitel 3, eine Risikoaversion des repräsentativen Investors geschätzt werden. Um dieses zu erreichen wird jedoch auf eine historische Zeitreihe zurückgegriffen und ein vollständig impliziter Rahmen verlassen. Für die Asset Allokation muss daher abgewägt werden, ob die Vorteile aus einem risikoadjustierten bzw. um Risikoprämien bereinigten Modell, denjenigen aus einem vollständig impliziten Ansatz überwiegen. 
Der wesentliche Vorteil aus der Risikoadjustierung im Gegensatz zu einem rein impliziten Ansatz ist eine geringere Verzerrung für die Prognose der realisierten Varianz und Schiefe. Der entscheidende Vorteil der risikoneutralen Momente wiederum ist, dass diese aus Daten von einem einzelnen Stichtag bestimmt werden und in der Konsequenz die Asset Allokation auf einem vollständig impliziten Ansatz basiert.

Im folgenden Kapitel, welches die taktische Asset Allokation untersucht, wird ein vollständig impliziter Ansatz gewählt. Die Vorteile aus der Risikoadjustierung gegenüber einem rein impliziten Ansatz überzeugen nicht und die optimale Asset Allokation wird somit ohne die Verwendung historischer Zeitreihen auf Basis von Daten eines einzelnen Stichtages gelöst. 


\section{Kapitel 5}

\section{Taktische Asset Allokation mit impliziten höheren Momenten}

\section{$5.1 \quad$ Einführung}

${ }^{1}$ Die taktische Asset Allokation untersucht die Aufteilung von Vermögen innerhalb einer Anlageklasse. Ein klassisches Anwendungsgebiet ist die Auswahl und Gewichtung unterschiedlicher Titel in einem Aktienmarkt und die Zusammenstellung eines entsprechenden Portfolios. Die taktische Asset Allokation ist abzugrenzen von der strategischen Asset Allokation aus Kapitel 3, bei welcher der Fokus auf der Gewichtung unterschiedlicher Anlageklassen wie Anleihen und Rohstoffe im Portfolio liegt. Eine "taktische" Fragestellung wäre in diesem Zusammenhang, welche Anleihe zum Beispiel unter Durationsgesichtspunkten stärker im Portfolio gewichtet wird oder welche expliziten Rohstoffe, wie Rohöl oder Gold, im Portfolio berücksichtigt werden. Die passiven und aktiven Strategien aus der taktischen Asset Allokation sind in der Praxis von großer Bedeutung, da sie die Grundlage für die Zusammensetzung von Indices bilden.

Die Anwendung impliziter Informationen im Rahmen der taktischen Asset Allokation mit höheren Momenten wurde in der Literatur bisher noch nicht betrachtet. Der Fokus bisheriger Forschung lag im Rahmen der Asset Allokation mit impliziten Informationen auf Investoren mit $\mu-\sigma$ Nutzenfunktionen und im Rahmen der Asset Allokation mit höheren Momenten auf der Auswertung von Schätzern auf Basis historischer Renditezeitreihen. Dieses Kapitel schließt diese Lücke und ist beiden Literatursträngen zuzuordnen.

$1 \quad$ Teile dieses Kapitels verwenden Resultate aus dem folgenden, in English verfassten Working Paper: Brinkmann, Kempf und Korn (2013): "Foward-Looking Measures of Higher Order Dependencies with an Application to Portfolio Selection", Working Paper Georg-August-Universität Göttingen und Centre for Financial Research Cologne (CFR). 
Martellini und Ziemann (2010) untersuchen die taktische Asset Allokation mit höheren Momenten auf Basis historischer Renditezeitreihen. Der spezielle Fokus der Arbeit ist der Umgang mit Schätzfehlern im Rahmen der Koschiefe- und Kokurtosismatrix. Die Autoren erweitern hierzu die Shrinkage Verfahren von Ledoit und Wolf (2003) und Ledoit und Wolf (2004) für die Kovarianzmatrix und konstruieren strukturierte Entsprechungen. In einer out-of-sample Studie wird ein erweiterter Minimum-Varianz-Ansatz unter Berücksichtigung der Koschiefe- und Kokurtosismatrix untersucht. Die Ergebnisse der Studie zeigen, dass, im Einklang zu dem Fall des Minimum-Varianz-Portfolios, die strukturierende Annahme eines Constant-Correlation-Ansatzes und die eines Ein-Faktor-Modells eine Verbesserung des Nutzens gegenüber dem unstrukturierten Stichprobenschätzer darstellt. Im Vergleich mit der Performance des Minimum-Varianz-Portfolios wird mit dem Portfolio auf Basis des um Koschiefe- und Kokurtosismatrix erweiterten Ansatzes ein geringerer Nutzen für einen monatlichen Anlagehorizont erzielt.

Die taktische Asset Allokation mit impliziten Informationen für Investoren mit $\mu$ - $\sigma$ Nutzenfunktionen untersuchen die Studien von DeMiguel, Plyakha, Uppal und Vilkov (2012) und Kempf, Korn und Saßning (2012). Erstere verfolgen insbesondere den Ansatz der parametrischen Portfolios nach Brandt, Santa-Clara und Valkanov (2009). Ausgehend von einem 1/N Portfolio zeigen die Autoren für die Mitglieder des S\&P 500, dass die Hinzunahme von impliziter Volatilität und Schiefe bei der Bildung der parametrischen Portfolios zu signifikant besseren Ergebnissen im Sinne der Sharpe Ratio gegenüber passiven und historischen Benchmark Strategien führen. Um Risikoprämien in Optionspreisen, wie die Varianz-Risikoprämie, zu berücksichtigen, greifen DeMiguel, Plyakha, Uppal und Vilkov (2012) auf historische Daten zurück und verwenden somit keinen vollständig impliziten Ansatz.

Kempf, Korn und Saßning (2012) hingegen verzichten auf die Berücksichtigung etwaiger Prämien und verfolgen einen Ansatz, welcher ausschließlich auf impliziten Informationen basiert. In ihrer Studie zeigen die Autoren für die Mitglieder des DJIA (Dow Jones Industrial Average), dass Minimum-Varianz-Portfolios auf Basis impliziter Informationen aktive und passive Benchmarks im Sinne der realisierten Volatilität statistisch signifikant schlagen. Diese Out-Performance wird im Speziellen in Phasen eines unruhigen Marktumfeldes erzielt.

Zur Untersuchung der taktischen Asset Allokation mit höheren Momenten unter Verwendung impliziter Informationen besteht eine besondere Herausforderung darin, die Erwartungswerte sowie die Kovarianz-, Koschiefe- und Kokurtosismatrix aus impliziten Informationen zu gewinnen. Die Vorgehensweise aus Kapitel 3, in welcher hierzu zunächst eine multidimensionale Dichte formuliert und die Momente aus der Integration dieser gewon- 
nen wurde, ist auf Grund der enormen Rechenintensität nicht ohne Weiteres auf mehrere riskante Anlagewerte übertragbar.

Die zentralen Momente können wie im vorherigen Kapitel mit Hilfe eines modellfreien Ansatzes bestimmt werden. Jedoch müssen zusätzlich auch Komomente, also die Abhängigkeitsstruktur, unter den Assets ermittelt werden, um insbesondere Diversifikationspotentiale richtig zu identifizieren und in der Asset Allokation zu berücksichtigen. Da Derivate, die auf Korrelationen basieren, wie Exchange-Optionen oder Quantos, nur begrenzt verfügbar sind, werden im Folgenden spezielle Strukturen unter den Assetrenditen unterstellt und mit Hilfe dieser und entsprechender Index-Optionen die Matrizen höherer Ordnung vollständig aus impliziten Informationen gewonnen. Konkret werden zwei Ansätze parallel getestet: zum einen die Annahme eines Constant-Correlation-Ansatzes, welche unterstellt, dass die Korrelationen unter den Assets konstant sind, und die Annahme eines Ein-Faktor-Modells, welche besagt, dass die Assetrenditen durch die Rendite eines Marktportfolios determiniert werden.

Der Constant-Correlation-Ansatz wird insbesondere in Skintzi und Refenes (2005), Driessen, Maenhout und Vilkov (2009) und Buss und Vilkov (2012) propagiert und in der Portfolioselektion mit impliziten Informationen in DeMiguel, Plyakha, Uppal und Vilkov (2012) angewendet. Hierbei wird aus der impliziten Volatilität einer Index-Option und der impliziten Volatilität der einzelnen Mitglieder eine konstante Korrelation gewonnen, welche wiederum zur Formulierung der vollständigen Kovarianzmatrix genutzt werden kann. Die Annahme eines Ein-Faktor-Modells wird unter anderem in Chang, Christoffersen, Jacobs und Vainberg (2011a) zur Gewinnung von impliziten Abhängigkeitsstrukturen verwendet und findet in Kempf, Korn und Saßning (2012) für die Portfolioselektion Anwendung. Die Kovarianzmatrix wird aus den Marktfaktoren ( $\beta$-Faktoren) und der Volatilität eines entsprechenden Indexes gewonnen. In beiden Ansätzen wird deutlich, dass zur Gewinnung einer impliziten Korrelation Optionen auf einen Index zur Verfügung stehen müssen. Dies ist darin begründet, dass in Index-Optionen neben den Informationen zu den einzelnen Assets zusätzlich auch deren Abhängigkeitsstruktur untereinander eingepreist ist und somit durch die unterschiedlichen Annahmen aus den Index-Optionen extrahiert werden kann.

Die erhaltenen impliziten Matrizen höherer Ordnung werden im Folgenden, wie in Kempf, Korn und Saßning (2012), ausschließlich unter dem risikoneutralen Maß gebildet. Sie sind daher nicht risikoadjustiert oder anderweitig in das physische Maß transformiert. Dies bedeutet insbesondere, dass sie nicht um Risikoprämien, wie der Varianz-Risikoprämie ${ }^{2}$

2 Vgl. z. B. Carr und Wu (2009). 
oder der Korrelations-Risikoprämie ${ }^{3}$, bereinigt sind. Eine Berücksichtigung dieser Prämien würde dazu führen, dass historische Daten zur Bestimmung der Matrizen verwendet werden müssen und somit kein vollständig impliziter Ansatz mehr gewährleistet ist.

Die Untersuchung der zentralen Forschungsfragen dieser Arbeit wird auf Basis eines Datensatzes für die Mitglieder des DJIA vorgenommen. Das Design der empirischen Studie orientiert sich an dem erweiterten Minimum-Varianz-Ansatz von Martellini und Ziemann (2010). Die von den Autoren entwickelten Anlagestrategien auf Basis historischer Renditezeitreihen werden übernommen und dienen neben passiven Anlageformen als Benchmarks zur Beurteilung der Performance der impliziten Strategien. Zur Frage, welchen Beitrag die höheren impliziten Momente Schiefe und Kurtosis für die out-of-sample Performance leisten, werden die Ergebnisse mit der Performance des Minimum-Varianz-Portfolios verglichen.

Der übrige Teil des Kapitels ist nun wie folgt gegliedert. Zunächst wird hergeleitet, wie aus rein impliziten Informationen Matrizen höherer Ordnung konstruiert werden können. Die unterstellten Annahmen und Modellimplikationen werden daraufhin anhand des Datensatzes für den DJIA empirisch untersucht. Es folgt die Studie zur Performance der Asset Allokations Strategien und dem Nutzen höherer Momente. Das Kapitel schließt mit einer Diskussion der Ergebnisse.

\subsection{Vollständig implizite Schätzer für Matrizen höherer Ordnung}

In diesem Abschnitt wird aufgezeigt, welche Strukturen bzw. Vereinfachungen es ermöglichen, eine Identifikation bzw. Schätzung der Matrizen $M_{2}, M_{3}$ und $M_{4}$ (vgl. Kapitel 2) vollständig aus impliziten Informationen zu erreichen. Hierzu werden die in der Einführung vorgestellten Ansätze, der Constant-Correlation-Ansatz und das Ein-Faktor-Modell, näher erläutert und diskutiert.

\subsubsection{Constant-Correlation-Ansatz}

Martellini und Ziemann (2010) leiten aus der Cauchy-Schwarz Ungleichung ${ }^{4}$ Korrelationen für die Komomente dritter und vierter Ordnung her. Diese Korrelationen sind aufgrund

$3 \quad$ Vgl. Driessen, Maenhout und Vilkov (2009).

4 Die Cauchy-Schwarz'sche Ungleichung für Zufallsvariablen $X, Y$ lautet:

$$
|E\{X Y\}| \leq \sqrt{E\left\{X^{2}\right\} E\left\{Y^{2}\right\}} .
$$


ihrer Definition beschränkt und liegen zwischen minus eins und eins. Mit Hilfe dieser Korrelationen für die Komomente höherer Ordnung wird folgend gezeigt, wie die Matrizen $M_{2}, M_{3}$ und $M_{4}$ aus impliziten Informationen identifiziert werden können. Die Herausforderung besteht insbesondere in der Identifikation der Komomente, da, wie im vorherigen Kapitel hergeleitet, die zentralen Momente der Log-Rendite Verteilung, wie Varianz, Schiefe und Kurtosis, modellfrei aus dem Ansatz von Bakshi, Kapadia und Madan (2003) aus Optionsmarktdaten bestimmt werden können.

Formal sei ein Portfolio ${ }^{5}$ aus $N$-Assets gegeben, auf welches Optionen gehandelt werden. Für das $i$-te Asset sei mit $\mu_{i}^{(n)}=E^{Q}\left\{\left(R_{i}-E^{Q}\left\{R_{i}\right\}\right)^{n}\right\}$ der $n$-te zentrale Moment der LogRendite Verteilung unter dem risikoneutralen Maß und mit $\omega_{i}$ das Gewicht im Portfolio bezeichnet. Mit dem Index $m$ sei der entsprechende Moment des Portfolios gekennzeichnet. ${ }^{6}$

\section{Implizite Kovarianzmatrix $M_{2}$}

Sei $M_{2}$ die Kovarianzmatrix der $N$-Assets. Dann werden die Einträge von $M_{2}$ gegeben durch

$$
M_{2_{i j}}=E\left\{\left(R_{i}-E\left\{R_{i}\right\}\right)\left(R_{j}-E\left\{R_{j}\right\}\right)\right\} .
$$

Wird unterstellt, dass die Korrelationen zwischen den Assets konstant sind, gilt

$$
M_{2_{i j}}=\rho^{M_{2}} \cdot \sqrt{\mu_{i}^{(2)} \mu_{j}^{(2)}} \quad \text { für alle } i \neq j .
$$

Die konstante Korrelation $\rho^{M_{2}}$ lässt sich mit Hilfe der Hilfsmatrix $\tilde{\Sigma} \in M_{N \times N}(\mathbb{R})$, welche durch das Produkt der Standardabweichungen auf den Nebendiagonalen und null auf der Hauptdiagonalen definiert ist, d. h.

$$
\tilde{\Sigma}_{i i}=0, \quad \tilde{\Sigma}_{i j}=\sqrt{\mu_{i}^{(2)} \mu_{j}^{(2)}}, \quad \text { für alle } i \neq j
$$

$5 \quad$ In dieser Studie sei das Portfolio typischerweise ein Index.

6 Die folgenden Darstellungen sind unter diesen Annahmen allgemeingültig und gelten daher nicht ausschließlich im Fall von impliziten Informationen. Um dies zu verdeutlichen, wird der Index $Q$ für das risikoneutrale Wahrscheinlichkeitsmaß vernachlässigt (vgl. Martellini und Ziemann (2010)). 
aus der Portfoliovarianz $\mu_{m}^{(2)}=\omega^{\text {tr }} M_{2} \omega$ gewinnen $^{7}$ :

$$
\rho^{M_{2}}=\frac{\mu_{m}^{(2)}-\sum_{i} \omega_{i}^{2} \mu_{i}^{(2)}}{\omega^{\operatorname{tr} \tilde{\Sigma} \omega}} .
$$

Die Korrelation $\rho^{M_{2}}$ lässt sich also durch die Portfoliovarianz $\mu_{m}^{(2)}$, die Gewichte $\omega_{1}, \ldots, \omega_{N}$ und die Varianzen der einzelnen Assets $\mu_{1}^{(2)}, \ldots, \mu_{N}^{(2)}$ bestimmen.

Die strukturierte (implizite) Kovarianzmatrix kann schließlich formuliert werden:

$$
M_{2_{C C}}^{i m p l}=\operatorname{diag}\left(\mu_{1}^{(2)}, \ldots, \mu_{N}^{(2)}\right)+\rho^{M_{2}} \cdot \tilde{\Sigma}
$$

\section{Implizite Koschiefematrix $M_{3}$}

Sei $M_{3}$ die Koschiefematrix der $N$-Assets. Dann werden die Einträge von $M_{3}$ gegeben durch:

$$
M_{3_{i j k}}=E\left\{\left(R_{i}-E\left\{R_{i}\right\}\right)\left(R_{j}-E\left\{R_{j}\right\}\right)\left(R_{k}-E\left\{R_{k}\right\}\right)\right\}
$$

Es wird unterstellt, dass die Korrelationen $\rho_{1}^{M_{3}}$ und $\rho_{1}^{M_{3}}$ für die Matrix $M_{3}$ gemäß Martellini und Ziemann (2010) zwischen den Assets konstant sind, d. h.

$$
\begin{aligned}
& M_{3_{i i j}}=\rho_{1}^{M_{3}} \cdot \sqrt{\mu_{i}^{(4)} \mu_{j}^{(2)}} \\
& M_{3_{i j k}}=\rho_{2}^{M_{3}} \cdot \sqrt{\mu_{k}^{(2)} \sqrt{\mu_{i}^{(4)} \mu_{j}^{(4)}}} \quad \text { für alle } i \neq j \neq k .
\end{aligned}
$$

Es wird weiter angenommen, dass zusätzlich $\rho_{1}^{M_{3}}=\rho_{2}^{M_{3}}=: \rho^{M_{3}}$ gilt. Dann lässt sich die Korrelation $\rho^{M_{3}}$ mit Hilfe der Hilfsmatrix $\tilde{S} \in M_{N \times N^{2}}(\mathbb{R})$, welche definiert wird durch

$$
\begin{aligned}
& \tilde{s}_{i i i}=0 \\
& \tilde{s}_{i i j}=\sqrt{\mu_{i}^{(4)} \mu_{j}^{(2)}} \\
& \tilde{s}_{i j k}=\sqrt{\mu_{k}^{(2)} \sqrt{\mu_{i}^{(4)} \mu_{j}^{(4)}}}, \quad \text { für alle } i \neq j \neq k
\end{aligned}
$$

aus der Portfolioschiefe $\mu_{m}^{(3)}=\omega^{t r} M_{3} \omega \otimes \omega$ gewinnen:

$$
\rho^{M_{3}}=\frac{\mu_{m}^{(3)}-\sum_{i} \omega_{i}^{3} \mu_{i}^{(3)}}{\omega^{t r} \tilde{S} \omega \otimes \omega} .
$$

Vgl. für diesen Ansatz z. B. Driessen, Maenhout und Vilkov (2009), Skintzi und Refenes (2005) und DeMiguel, Plyakha, Uppal und Vilkov (2012). 
Die Korrelation $\rho^{M_{3}}$ lässt sich also durch die Portfolioschiefe $\mu_{m}^{(3)}$, die Gewichte $\omega_{1}, \ldots, \omega_{N}$ und die Schiefen der einzelnen Assets $\mu_{1}^{(3)}, \ldots, \mu_{N}^{(3)}$ sowie durch die Varianzen $\mu_{1}^{(2)}, \ldots, \mu_{N}^{(2)}$ und die Kurtosin $\mu_{1}^{(4)}, \ldots, \mu_{N}^{(4)}$ der einzelnen Assets bestimmen.

Die strukturierte (implizite) Koschiefematrix kann nun formuliert werden als

$$
M_{3_{C C}}^{i m p l}=\operatorname{diag}\left(\mu_{1}^{(3)}, \ldots, \mu_{N}^{(3)}\right)+\rho^{M_{3}} \cdot \tilde{S},
$$

wobei diag in diesem Fall die $N \times N^{2}$ Matrix mit Einträgen in iii bezeichnet.

\section{Implizite Kokurtosismatrix $M_{4}$}

Analog sei $M_{4}$ die Kokurtosismatrix der $N$-Assets. Dann werden die Einträge von $M_{4}$ gegeben durch:

$$
M_{4_{i j k l}}=E\left\{\left(R_{i}-E\left\{R_{i}\right\}\right)\left(R_{j}-E\left\{R_{j}\right\}\right)\left(R_{k}-E\left\{R_{k}\right\}\right)\left(R_{l}-E\left\{R_{l}\right\}\right)\right\}
$$

Wird unterstellt, dass die Korrelationen $\rho_{1}^{M_{4}}, \rho_{2}^{M_{4}}, \rho_{3}^{M_{4}}$ und $\rho_{4}^{M_{4}}$ für die Matrix $M_{4}$ gemäß Martellini und Ziemann (2010) zwischen den Assets konstant sind, gilt

$$
\begin{aligned}
& M_{4_{i i i j}}=\rho_{1}^{M_{4}} \cdot \sqrt{\mu_{i}^{(6)} \mu_{j}^{(2)}} \\
& M_{4_{i i j j}}=\rho_{2}^{M_{4}} \cdot \sqrt{\mu_{i}^{(4)} \mu_{j}^{(4)}} \\
& M_{4_{i i j k}}=\rho_{3}^{M_{4}} \cdot \sqrt{\mu_{i}^{(4)} \sqrt{\mu_{j}^{(4)} \mu_{k}^{(4)}}} \\
& M_{4_{i j k l}}=\rho_{4}^{M_{4}} \cdot \sqrt{\sqrt{\mu_{i}^{(4)} \mu_{j}^{(4)}} \sqrt{\mu_{k}^{(4)} \mu_{l}^{(4)}}} \quad \text { für alle } i \neq j \neq k \neq l .
\end{aligned}
$$

Unter der Annahme, dass zusätzlich $\rho_{1}^{M_{4}}=\rho_{2}^{M_{4}}=\rho_{3}^{M_{4}}=\rho_{4}^{M_{4}}=: \rho^{M_{4}}$ gilt, lässt sich die Korrelation $\rho^{M_{4}}$ mit Hilfe der Hilfsmatrix $\tilde{K} \in M_{N \times N^{3}}(\mathbb{R})$, welche definiert wird durch

$$
\begin{aligned}
& \tilde{k}_{i i i i}=0 \\
& \tilde{k}_{i i i j}=\sqrt{\mu_{i}^{(6)} \mu_{j}^{(2)}} \\
& \tilde{k}_{i i j j}=\sqrt{\mu_{i}^{(4)} \mu_{j}^{(4)}} \\
& \tilde{k}_{i i j k}=\sqrt{\mu_{i}^{(4)} \sqrt{\mu_{j}^{(4)} \mu_{k}^{(4)}}} \\
& \tilde{k}_{i j k l}=\sqrt{\sqrt{\mu_{i}^{(4)} \mu_{j}^{(4)}} \sqrt{\mu_{k}^{(4)} \mu_{l}^{(4)}}} \quad \text { für alle } i \neq j \neq k \neq l
\end{aligned}
$$


aus der Portfoliokurtosis $\mu_{m}^{(4)}=\omega^{\text {tr }} M_{4} \omega \otimes \omega \otimes \omega$ gewinnen:

$$
\rho^{M_{4}}=\frac{\mu_{m}^{(4)}-\sum_{i} \omega_{i}^{4} \mu_{i}^{(4)}}{\omega^{t r} \tilde{K} \omega \otimes \omega \otimes \omega} .
$$

Die Korrelation $\rho^{M_{4}}$ lässt sich also durch die Portfoliokurtosis $\mu_{m}^{(4)}$, die Gewichte $\omega_{1}, \ldots, \omega_{N}$ und den Kurtosin der einzelnen Assets $\mu_{1}^{(4)}, \ldots, \mu_{N}^{(4)}$ sowie der Varianzen $\mu_{1}^{(2)}, \ldots, \mu_{N}^{(2)}$ und der zentralen sechsten Momente $\mu_{1}^{(6)}, \ldots, \mu_{N}^{(6)}$ bestimmen.

Die strukturierte (implizite) Kokurtosismatrix kann nun formuliert werden:

$$
M_{4_{C C}}^{i m p l}=\operatorname{diag}\left(\mu_{1}^{(4)}, \ldots, \mu_{N}^{(4)}\right)+\rho^{M_{4}} \cdot \tilde{K}
$$

wobei diag in diesem Fall die $N \times N^{3}$ Matrix mit Einträgen in iiii bezeichnet.

Durch die obigen Annahmen an die Struktur der Komomente basieren die benötigten Parameter zur Formulierung der Matrizen $M_{2_{C C}}^{i m p l}, M_{3_{C C}}^{i m p l}$ und $M_{4_{C C}}^{i m p l}$ ausschließlich auf den zentralen Momenten der einzelnen Assets und des Indexes und können somit aus Daten von einem einzelnen Stichtag identifiziert werden. Es werden keine weiteren historischen Daten benötigt, d. h. die Darstellung basiert folglich auf einem vollständig impliziten Ansatz.

Zur Unterstützung der Annahme, die unterschiedlichen Korrelationen in den Matrizen höherer Ordnung seien gleich, zeigt Tabelle 5.1, in welcher Anzahl diese in den Matrizen vorkommen. Dabei wird aufgeschlüsselt, wie oft die entsprechenden Komomente in den einzelnen Blöcken $(N \times N$ Matrizen $)$ und in der gesamten Matrix vorkommen. ${ }^{8}$

8 Vgl. hierzu die Interpretation der Koschiefe- und Kokurtosismatrix aus Kapitel 2 als aneinandergereihte $N \times N$ Matrizen. 
Tabelle 5.1: Anzahl der Komomente und der Korrelationen höherer Ordnung in den Matrizen $M_{2}, M_{3}$ und $M_{4}$

\begin{tabular}{|c|c|c|c|}
\hline Varianz $M_{2}$ & \multicolumn{2}{|c|}{1 Block } & $N^{2}-N$ Komomente \\
\hline$\rho^{M_{2}}$ & \multicolumn{2}{|c|}{$N(N-1)$} & $N(N-1)$ \\
\hline Schiefe $M_{3}$ & \multicolumn{2}{|c|}{$N$ Blöcke } & $N^{3}-N$ Komomente \\
\hline $\begin{array}{l}\rho_{1}^{M_{3}} \\
\rho_{2}^{M_{3}}\end{array}$ & \multicolumn{2}{|c|}{$\begin{array}{c}3(N-1) \\
(N-1)(N-2)\end{array}$} & $\begin{array}{l}3 N(N-1) \\
N(N-1)(N-2)\end{array}$ \\
\hline Kurtosis $M_{4}$ & $\begin{array}{l}\quad N^{2} \\
\quad i=j \\
N \text { Blöcke }\end{array}$ & $\begin{array}{l}\text { löcke } \\
\qquad \begin{array}{l}i \neq j \\
N(N-1) \text { Blöcke }\end{array}\end{array}$ & $N^{4}-N$ Komomente \\
\hline $\begin{array}{l}\rho_{1}^{M_{4}} \\
\rho_{2}^{M_{4}} \\
\rho_{3}^{M_{4}} \\
\rho_{4}^{M_{4}}\end{array}$ & $\begin{array}{c}2(N-1) \\
(N-1) \\
(N-1)(N-2) \\
-\end{array}$ & $\begin{array}{c}2 \\
2 \\
5(N-2) \\
(N-2)(N-3)\end{array}$ & $\begin{array}{l}4 N(N-1) \\
3 N(N-1) \\
6 N(N-1)(N-2) \\
N(N-1)(N-2)(N-3)\end{array}$ \\
\hline
\end{tabular}

Diese Tabelle zeigt, in welcher Anzahl die Komomente und die Korrelationen höherer Ordnung in den Matrizen $M_{2}, M_{3}$ und $M_{4}$ auftreten. Mit $N$ sei die Anzahl der Assets bezeichnet.

Es lässt sich für die Matrizen $M_{3}$ und $M_{4}$ erkennen, dass für große $N$ die Komomente in den Matrizen ausschließlich durch die Korrelationen $\rho_{2}^{M_{3}}$ und $\rho_{4}^{M_{4}}$ bestimmt werden. Für $N \rightarrow \infty$ konvergieren die relativen Häufigkeiten für diese Werte sogar gegen eins, die der übrigen Korrelationen gegen null.

\subsubsection{Annahme eines Ein-Faktor-Modells ${ }^{9}$}

Als weiterer Ansatz zur Formulierung der Matrizen $M_{2}, M_{3}$ und $M_{4}$ aus rein impliziten Informationen kann die Annahme eines Ein-Faktor-Modells bemüht werden. Hierzu wird unterstellt, dass die Renditen der Assets einem verallgemeinerten Marktmodell mit zeitva-

$9 \quad$ Dieser Abschnitt orientiert sich an Kapitel 3.4.2 aus Saßning (2012) und Martellini und Ziemann (2010). 
riablen Koeffizienten im Sinne von Sharpe (1963) folgen. Im Speziellen seien die Renditen der Assets beschrieben durch ${ }^{10}$

$$
R_{i t}=\alpha_{i t}+\beta_{i t} R_{M t}+\epsilon_{i t}
$$

wobei $\alpha_{i t}$ den Achsenabschnitt, $\beta_{i t}$ die Steigung und $\epsilon_{i t}$ die idiosynkratischen Störterme zum Zeitpunkt $t$ für das $i$-te Asset bezeichnen. Es wird angenommen, dass im Querschnitt die Störterme $\epsilon_{i t}$ und $\epsilon_{j t}$ für $i \neq j$ sowie $\epsilon_{i t}$ und $R_{M t}$ stochastisch unabhängig sind. Der Erwartungswert von $\epsilon_{i t}$ ist null.

Unter diesen Annahmen lassen sich die Matrizen $M_{2}, M_{3}$ und $M_{4}$ wie folgt darstellen:11

$$
\begin{aligned}
M_{2} & =E\left\{\left(\beta\left(R_{M}-\mu_{m}^{(1)}\right)+\epsilon\right)\left(\beta\left(R_{M}-\mu_{m}^{(1)}\right)+\epsilon\right)^{t r}\right\} \\
& =\left(\beta \beta^{t r}\right) \mu_{m}^{(2)}+\Psi
\end{aligned}
$$

wobei $\beta$ und $\epsilon$ jeweils die $N \times 1$ - Vektoren $\operatorname{der} \beta_{i}$-Faktoren und der Störterme $\epsilon_{i}$ bezeichnen. $\Psi \in M_{N \times N}(\mathbb{R})$ ist die Kovarianzmatrix der idiosynkratischen Störterme $\epsilon_{i}$ und aufgrund der paarweisen Unkorreliertheit (bzw. der stochastischen Unabhängigkeit) der Störterme gilt

$$
\begin{aligned}
\Psi_{i i} & =\mu_{\epsilon_{i}}^{(2)} \\
\Psi_{i j} & =0, \quad \text { für alle } i \neq j .
\end{aligned}
$$

Die Einträge von $M_{2}$ werden gegeben durch

$$
\begin{aligned}
& M_{2_{i i}}=\mu_{i}^{(2)} \\
& M_{2_{i j}}=\beta_{i} \beta_{j} \cdot \mu_{m}^{(2)} \quad \text { für alle } i \neq j .
\end{aligned}
$$

Die Koschiefematrix kann in ähnlicher Weise dargestellt werden.

$$
\begin{aligned}
M_{3} & =E\left\{\left(\beta\left(R_{M}-\mu_{m}^{(1)}\right)+\epsilon\right)\left(\beta\left(R_{M}-\mu_{m}^{(1)}\right)+\epsilon\right)^{\operatorname{tr}} \otimes\left(\beta\left(R_{M}-\mu_{m}^{(1)}\right)+\epsilon\right)^{t r}\right\} \\
& =\left(\beta \beta^{t r} \otimes \beta^{t r}\right) \mu_{m}^{(3)}+\Phi
\end{aligned}
$$

10 Vgl. auch Kempf, Korn und Saßning (2012).

11 Diese Darstellung ist unter diesen Annahmen allgemeingültig und gilt daher nicht ausschließlich im Fall von impliziten Informationen. Um dies zu verdeutlichen, wird der Index $Q$ für das Wahrscheinlichkeitsmaß vernachlässigt (vgl. Martellini und Ziemann (2010)). Zur Übersichtlichkeit sei auch der Index $t$ vernachlässigt, welcher verdeutlichen würde, dass die Matrizen zu jedem Zeitpunkt $t$ neu definiert werden. 
wobei $\Phi \in M_{N \times N^{2}}(\mathbb{R})$ die Koschiefematrix der idiosynkratischen Störterme $\epsilon_{i}$ bezeichnet. Da die Störterme als paarweise stochastisch unabhängig angenommen sind, sind auch die Komomente der höheren Momente gleich null. Es gilt

$$
\begin{aligned}
\Phi_{i i i} & =\mu_{\epsilon_{i}}^{(3)} \\
\Phi_{i i j} & =0 \\
\Phi_{i j k} & =0, \quad \text { für alle } i \neq j \neq k .
\end{aligned}
$$

Zusammengefasst werden die Einträge von $M_{3}$ gegeben durch

$$
\begin{aligned}
& M_{3_{i i i}}=\mu_{i}^{(3)} \\
& M_{3_{i i j}}=\beta_{i}^{2} \beta_{j} \cdot \mu_{m}^{(3)} \\
& M_{3_{i j k}}=\beta_{i} \beta_{j} \beta_{k} \cdot \mu_{m}^{(3)}, \quad \text { für alle } i \neq j \neq k .
\end{aligned}
$$

Die Kokurtosismatrix muss gesondert betrachtet werden, da selbst bei der unterstellten stochastischen Unabhängigkeit auch Produkte aus der Varianz des Marktes $\mu_{m}^{(2)}$ und der Varianz der Störterme $\mu_{\epsilon_{i}}^{(2)}$ berücksichtig werden müssen.

$$
\begin{aligned}
M_{4}= & E\left\{\left(\beta\left(R_{M}-\mu_{m}^{(1)}\right)+\epsilon\right)\left(\beta\left(R_{M}-\mu_{m}^{(1)}\right)+\epsilon\right)^{t r}\right. \\
& \left.\otimes\left(\beta\left(R_{M}-\mu_{m}^{(1)}\right)+\epsilon\right)^{t r} \otimes\left(\beta\left(R_{M}-\mu_{m}^{(1)}\right)+\epsilon\right)^{t r}\right\} \\
= & \left(\beta \beta \otimes \beta^{t r} \otimes \beta^{t r}\right) \mu_{m}^{(4)}+\Upsilon
\end{aligned}
$$

wobei $\Upsilon \in M_{N \times N^{3}}(\mathbb{R})$ neben der Kurtosis der Störterme auch die Produkte aus den Varianzen berücksichtigt. Die Einträge von $\Upsilon$ werden gegeben durch

$$
\begin{aligned}
& \Upsilon_{i i i i}=6 \beta_{i}^{2} \mu_{m}^{(2)} \mu_{\epsilon_{i}}^{(2)}+\mu_{\epsilon_{i}}^{(4)} \\
& \Upsilon_{i i i j}=3 \beta_{i} \beta_{j} \mu_{m}^{(2)} \mu_{\epsilon_{i}}^{(2)} \\
& \Upsilon_{i i j j}=\beta_{i}^{2} \mu_{m}^{(2)} \mu_{\epsilon_{j}}^{(2)}+\beta_{j}^{2} \mu_{m}^{(2)} \mu_{\epsilon_{i}}^{(2)}+\mu_{\epsilon_{i}}^{(2)} \mu_{\epsilon_{j}}^{(2)} \\
& \Upsilon_{i i j k}=\beta_{j} \beta_{k} \mu_{m}^{(2)} \mu_{\epsilon_{i}}^{(2)} \\
& \Upsilon_{i j k l}=0, \quad \text { für alle } i \neq j \neq k \neq l .
\end{aligned}
$$

Die Einträge der Matrix $M_{4}$ werden schließlich gegeben durch

$$
\begin{aligned}
& M_{4_{i i i i}}=\mu_{i}^{(4)} \\
& M_{4_{i i i j}}=\beta_{i}^{3} \beta_{j} \cdot \mu_{m}^{(4)}+3 \beta_{i} \beta_{j} \mu_{m}^{(2)} \mu_{\epsilon_{i}}^{(2)} \\
& M_{4_{i i j j}}=\beta_{i}^{2} \beta_{j}^{2} \cdot \mu_{m}^{(4)}+\beta_{i}^{2} \mu_{m}^{(2)} \mu_{\epsilon_{j}}^{(2)}+\beta_{j}^{2} \mu_{m}^{(2)} \mu_{\epsilon_{i}}^{(2)}+\mu_{\epsilon_{i}}^{(2)} \mu_{\epsilon_{j}}^{(2)} \\
& M_{4_{i i j k}}=\beta_{i}^{2} \beta_{j} \beta_{k} \cdot \mu_{m}^{(4)}+\beta_{j} \beta_{k} \mu_{m}^{(2)} \mu_{\epsilon_{i}}^{(2)} \\
& M_{4_{i j k l}}=\beta_{i} \beta_{j} \beta_{k} \beta_{l} \cdot \mu_{m}^{(4)}, \quad \text { für alle } i \neq j \neq k \neq l .
\end{aligned}
$$


Zur Bestimmung der Matrizen $M_{2}, M_{3}$ und $M_{4}$ aus einem vollständig impliziten Ansatz müssen neben den zentralen Momenten Varianz, Schiefe und Kurtosis der einzelnen Assets und des Marktes insbesondere die $\beta$-Faktoren und die Varianz der Störterme bestimmt werden. Die zentralen Momente können wie schon im Constant-Correlation-Ansatz aus dem modellfreien Ansatz nach Bakshi, Kapadia und Madan (2003) (vgl. Kapitel 4) abgeleitet werden. Für die verbleibenden Größen ( $\beta$-Faktoren und Varianz der Störterme) wird das Faktormodell und dessen Modellimplikationen mit Hilfe weiterer Annahmen erneut bemüht.

Im Ein-Faktor-Modell kann die Varianz eines einzelnen Assets in ihren systematischen und idiosynkratischen Anteil zerlegt werden:

$$
\mu_{i}^{(2)}=\beta_{i}^{2} \mu_{m}^{(2)}+\mu_{\epsilon_{i}}^{(2)}
$$

Aus dieser Darstellung kann die Varianz der Störterme durch Umstellung der Gleichung direkt gewonnen werden:

$$
\mu_{\epsilon_{i}}^{(2)}=\mu_{i}^{(2)}-\beta_{i}^{2} \mu_{m}^{(2)}
$$

Es verbleibt die Identifizierung der $\beta$-Faktoren aus dem Modell. Hierzu wird die zusätzliche Annahme getroffen, dass zum Zeitpunkt $t$ der systematische Anteil der Varianz an der Gesamtvarianz von $R_{i t}$ für alle Assets im Querschnitt $c_{t}$ beträgt. Die idiosynkratische Varianz $\mu_{\epsilon_{i}}^{(2)}$ kann dann als Anteil $\left(1-c_{t}\right)$ der Gesamtvarianz von $R_{i t}$ dargestellt werden: ${ }^{12}$

$$
\mu_{\epsilon_{i}}^{(2)}=\left(1-c_{t}\right) \mu_{i}^{(2)}
$$

Der Anteil $c_{t}$ ist also zeitvariabel, aber dennoch unabhängig von $i$. Durch Einsetzen ergibt sich für $\beta_{i}$

$$
\beta_{i}=\sqrt{\frac{c_{t} \mu_{i}^{(2)}}{\mu_{m}^{(2)}}} .
$$

Der Anteil $c_{t}$ kann wiederum aus der Eigenschaft des Markportfolios, dass die gewichtete Summe der $\beta$-Faktoren eins ist, $\sum_{i} \omega_{i} \beta_{i}=1$, gewonnen werden:

$$
c_{t}=\frac{\mu_{m}^{(2)}}{\left(\sum_{i} \omega_{i} \sqrt{\mu_{i}^{(2)}}\right)^{2}},
$$

12 Vgl. hierzu Saßning (2012), Baule, Korn und Saßning (2013) und Kempf, Korn und Saßning (2012). 
womit schließlich eingesetzt für die $\beta$-Faktoren gilt, dass diese aus der gewichteten Summe der impliziten Volatilitäten identifiziert werden können:

$$
\beta_{i}=\frac{\sqrt{\mu_{i}^{(2)}}}{\sum_{i} \omega_{i} \sqrt{\mu_{i}^{(2)}}}
$$

Durch die Annahme, dass der Anteil der systematischen Varianz an der Gesamtvarianz für sämtliche Assets übereinstimmt, lassen sich die $\beta$-Faktoren und somit auch die Matrizen $M_{2}, M_{3}$ und $M_{4}$ ausschließlich durch implizite Informationen und den Gewichten der Assets im Marktportfolio bestimmen. Dieser Ansatz stellt somit einen weiteren vollständig impliziten Ansatz zur Identifikation der strukturierten Matrizen $M_{2_{S F}}^{i m p l}, M_{3_{S F}}^{i m p l}$ und $M_{4_{S F}}^{i m p l}$ dar.

Die vorgestellte Identifikation der $\beta$-Faktoren basiert ausschließlich auf der Verwendung von impliziten Varianzen. Die Faktoren können mit vergleichbaren Argumenten auch auf Basis der Schiefe und Kurtosis identifiziert werden. ${ }^{13}$ Baule, Korn und Saßning (2013) zeigen jedoch, dass diese weiteren impliziten $\beta$-Faktoren in Hinsicht auf Prognosequalität und Verzerrung realisierter $\beta$-Faktoren keine Verbesserungen gegenüber der vorgestellten Identifikation auf Basis der Varianz darstellen. Aufgrund dessen wird im Folgenden darauf verzichtet, weitere implizite $\beta$-Faktoren in der empirischen Studie zu berücksichtigen und zu untersuchen.

\subsection{Daten}

Die Untersuchung der taktischen Asset Allokation für Investoren mit einem monatlichen Anlagehorizont erfolgt auf Basis eines Datensatzes für die Mitglieder des DJIA. Die Investoren haben die Möglichkeit, ihr Vermögen auf die 30 Unternehmen zu verteilen und ein Portfolio zu bilden. Aktiensplits und Dividenden werden in der Anlage berücksichtigt. Tabelle 5.2 zeigt die Unternehmen (Ticker), welche in dem betrachteten Zeitraum Mitglied des DJIA waren.

13 Vgl. Chang, Christoffersen, Jacobs und Vainberg (2011a) und Saßning (2012). 
Tabelle 5.2: Zeitraum und Ticker der Unternehmen im DJIA

\begin{tabular}{lclc}
\hline Ticker & Zeitraum & Ticker & Zeitraum \\
\hline UTX & $01 / 98-10 / 10$ & TRV & $06 / 09-10 / 10$ \\
CAT & $01 / 98-10 / 10$ & UK & $01 / 98-11 / 99$ \\
MCD & $01 / 98-10 / 10$ & MO & $01 / 98-02 / 08$ \\
XOM & $01 / 98-10 / 10$ & CVX & $01 / 98-11 / 99$ \\
WMT & $01 / 98-10 / 10$ & T & $11 / 05-10 / 10$ \\
IBM & $01 / 98-10 / 10$ & CSCO & $06 / 09-10 / 10$ \\
MMM & $01 / 98-10 / 10$ & HON & $01 / 98-02 / 08$ \\
HPQ & $01 / 98-10 / 10$ & VZ & $04 / 04-10 / 10$ \\
JNJ & $01 / 98-10 / 10$ & KFT & $09 / 08-10 / 10$ \\
PG & $01 / 98-10 / 10$ & CVX & $02 / 08-10 / 10$ \\
AXP & $01 / 98-10 / 10$ & IP & $01 / 98-04 / 04$ \\
JPM & $01 / 98-10 / 10$ & MSFT & $11 / 99-10 / 10$ \\
BA & $01 / 98-10 / 10$ & HD & $11 / 99-10 / 10$ \\
KO & $01 / 98-10 / 10$ & INTC & $11 / 99-10 / 10$ \\
DIS & $01 / 98-10 / 10$ & PFE & $04 / 04-10 / 10$ \\
AA & $01 / 98-10 / 10$ & SBC & $11 / 99-11 / 05$ \\
MRK & $01 / 98-10 / 10$ & EK & $01 / 98-04 / 04$ \\
DD & $01 / 98-10 / 10$ & C & $01 / 98-06 / 09$ \\
GE & $01 / 98-10 / 10$ & T & $01 / 98-04 / 04$ \\
& & S & $01 / 98-11 / 99$ \\
& & MTLQQ & $01 / 98-06 / 09$ \\
& & GT & $01 / 98-11 / 99$ \\
& & BAC & $02 / 08-10 / 10$ \\
& & AIG & $04 / 04-09 / 08$ \\
\hline
\end{tabular}

Diese Tabelle zeigt die Ticker der Unternehmen, welche Mitglieder des DJIA im out-of-sample Zeitraum waren. Die linke Reihe bezieht sich auf Unternehmen, die während des gesamten Zeitraumes Bestandteil des DJIA waren. Die rechte Reihe zeigt nicht-permanente Mitglieder.

Für die empirische Studie wird die Erwartungsbildung der zukünftigen Renditen, welche die optimale Asset Allokation determiniert, anhand von impliziten Informationen aus Optionsmarktdaten und historischen Renditezeitreihen erfolgen. Für die entsprechenden Methodiken müssen verschiedene Daten verfügbar sein.

Zur Identifikation der impliziten Informationen werden neben Daten von Optionen auf die Mitglieder des DJIA auch solche von Optionen auf den Index selbst benötigt. Die entsprechenden Daten liegen in der Optionsdatenbank IvyDB von Optionmetrics für den Zeitraum Januar 1998 bis Oktober 2010 vor, welche sich auf an der CBOE gehandelte Optionen beziehen. Für die Studie werden insbesondere die zur Verfügung gestellten Volatilitätsoberflächen verwendet. Im Folgenden wird als Stichtag für jeden Monat (Beobachtungszeitpunkt) der erste Montag nach dem dritten Freitag verwendet. Dies entspricht dem ersten Handelstag nach dem Verfallstermin von Optionen an der CBOE und garantiert eine möglichst gute Approximation der Laufzeit der Optionen von einem Monat. 
Historische Renditezeitreihen stammen aus Datastream, wobei die historischen Kurse um Aktiensplits und Dividenden bereinigt wurden. Der out-of-sample Zeitraum der Studie beträgt Februar 1998 bis November 2010, dies entspricht (bei monatlicher Betrachtung) 153 Beobachtungen. Als risikofreier Zinssatz wird der durch die IvyDB bereitgestellte Zinssatz $r$ genutzt. Die Zinsstruktur wird hierbei aus dem BBA Libor Zinssatz und den Settlement Preisen von CME Eurodollar Futures berechnet. ${ }^{14}$

Zur weiteren Robustheit der Studie werden neben dem vollständigen Datensatz von $N=30$ Assets auch zwei Subportfolios mit $N=10$ und $N=20$ Assets untersucht. Hierzu werden in jedem Beobachtungszeitpunkt $t$ die nach Marktkapitalisierung größten 10 bzw. 20 Unternehmen im Index ausgewählt und als gesonderte Portfolios untersucht. Für passive Strategien werden die entsprechenden Gewichte normiert, um zu gewährleisten, dass sich die Gewichte in den Subportfolios zu eins summieren.

\subsection{Methodik der empirischen Studie}

Dieser Abschnitt stellt den Aufbau und das Vorgehen der empirischen Studie vor. Zunächst wird das Asset Allokationsproblem auf Basis impliziter Informationen für die betrachteten Investoren der Studie beschrieben. Es folgt die Vorstellung der Benchmark Strategien und der Performance-Kennzahlen.

\subsubsection{Bestimmung der optimalen Asset Allokation}

Für die empirische Studie werden, wie in Kapitel 2 beschrieben, Investoren mit CRRA Nutzenfunktionen mit Risikoaversionsparameter $\gamma=2,4,6$ und 8 untersucht. Der Anlagehorizont der Investoren beträgt einen Monat und die Investoren haben nicht die Möglichkeit, Short-Positionen bei ihrer Asset Allokation einzugehen und unterliegen somit einer strikten Leerverkaufsbeschränkung.

Zur Bestimmung der optimalen Asset Allokation bzw. der optimalen Gewichte im Portfolio wird dem Ansatz von Martellini und Ziemann (2010) gefolgt. Dieser kann als erweiterter Minimum-Varianz-Ansatz verstanden werden. Hierzu wird das Optimierungsproblem (2.7)

$14 \quad$ Vgl. Optionmetrics Handbuch. 
für die Risikoaversion $\gamma>0$ betrachtet, wobei zusätzlich der Erwartungswert konstant null gesetzt wird. Es verbleibt folgendes Asset Allokationsproblem:

$$
\begin{aligned}
\underset{\omega \in \mathbb{R}^{N}, \text { s.t. } \sum_{i=1}^{N} \omega_{i}=1}{\arg \max _{\omega}}[ & -\frac{\gamma}{2} \omega^{t r} M_{2} \omega+\frac{\gamma(\gamma+1)}{6} \omega^{t r} M_{3}(\omega \otimes \omega) \\
& \left.-\frac{\gamma(\gamma+1)(\gamma+2)}{24} \omega^{t r} M_{4}(\omega \otimes \omega \otimes \omega)\right] .
\end{aligned}
$$

Die Matrizen $M_{2}, M_{3}$ und $M_{4}$ werden zu jedem Stichtag je nach Methodik durch die impliziten Matrizen $M_{2_{C C}}^{i m p l}, M_{3_{C C}}^{i m p l}$ und $M_{4_{C C}}^{i m p l}$ bzw. $M_{2_{S F}}^{i m p l}, M_{3_{S F}}^{i m p l}$ und $M_{4_{S F}}^{i m p l}$ ersetzt und das Optimierungsproblem (5.1) wird mit Hilfe eines nicht-linearen Algorithmus ${ }^{15}$ unter der zusätzlichen Annahme der strikten Leerverkaufsbeschränkung gelöst.

Die Bestimmung der impliziten Matrizen $M_{2_{C C}}^{i m p l}, M_{3_{C C}}^{i m p l}$ und $M_{4_{C C}}^{i m p l}$ bzw. $M_{2_{S F}}^{i m p l}, M_{3_{S F}}^{i m p l}$ und $M_{4_{S F}}^{i m p l}$ basiert auf den zentralen Momenten der Mitglieder des Indexes sowie derjenigen des Indexes selbst. Die Berechnung dieser erfolgt mit Hilfe der modellfreien Methodik aus Bakshi, Kapadia und Madan $(2003)^{16}$ (vgl. Kapitel 4). ${ }^{17}$ Die erhaltenen optimalen

15 R-Funktion: nlm. Als Initial Portfolio für die Optimierung (Startwert) werden die Gewichte des Minimum-Varianz-Portfolios mit Leerverkaufsbeschränkungen als 'Best-Guess" gewählt. Die Bestimmung dieses erfolgt mit Hilfe des Critical Line Algorithmusses (Vgl. Saßning (2012)).

16 Die Methodik aus Bakshi, Kapadia und Madan (2003) bestimmt die Momente der risikoneutralen Log-Rendite Verteilung. In der Portfolioselektion werden jedoch diskrete Renditen benötigt. Im Folgenden wird daher davon ausgegangen, dass die Momente der Log-Rendite Verteilung als Approximation der Momente der diskreten Rendite Verteilung verwendet werden können. Aus Gründen der Konsistenz werden die Benchmark Strategien auf Basis historischer Log-Renditezeitreihen gebildet. Ein Übergang zu diskreten Renditen kann ohne weitere Annahmen über das Spanning Argument aus Carr und Madan (2001) geleistet werden (eine explizite Formulierung der diskreten impliziten Momente findet sich z. B. in Chang, Christoffersen und Jacobs (2011b)).

17 Korrolar 2 zeigt leidglich die Werte bis zur vierten Ordnung. Für diese Studie wird zusätzlich das sechste Moment benötigt. Dieses kann analog bestimmt werden, in dem für die Funktionen $H_{5}^{\prime \prime}\left(S_{t+\tau}\right)$ und $H_{6}^{\prime \prime}\left(S_{t+\tau}\right)$ die entsprechenden Kontrakte gebildet werden:

$$
\begin{aligned}
E^{Q}\left\{e^{-r \tau} H_{5}^{\prime \prime}\left(S_{t+\tau}\right)\right\}= & \int_{S_{t}}^{\infty} \frac{20\left(\log \left(\frac{K}{S_{t}}\right)\right)^{3}-5\left(\log \left(\frac{K}{S_{t}}\right)\right)^{4}}{K^{2}} C(t, \tau, K) d K \\
& -\int_{0}^{S_{t}} \frac{20\left(\log \left(\frac{S_{t}}{K}\right)\right)^{3}+5\left(\log \left(\frac{S_{t}}{K}\right)\right)^{4}}{K^{2}} P(t, \tau, K) d K \\
E^{Q}\left\{e^{-r \tau} H_{6}^{\prime \prime}\left(S_{t+\tau}\right)\right\}= & \int_{S_{t}}^{\infty} \frac{30\left(\log \left(\frac{K}{S_{t}}\right)\right)^{4}-6\left(\log \left(\frac{K}{S_{t}}\right)\right)^{5}}{K^{2}} C(t, \tau, K) d K \\
& +\int_{0}^{S_{t}} \frac{30\left(\log \left(\frac{S_{t}}{K}\right)\right)^{4}+6\left(\log \left(\frac{S_{t}}{K}\right)\right)^{5}}{K^{2}} P(t, \tau, K) d K .
\end{aligned}
$$


Portfolios für die impliziten Strategien werden am Ende des Anlagehorizonts bzgl. der Performancemetriken in Abschnitt 5.4.3 in einem out-of-sample Rahmen ausgewertet.

\subsubsection{Benchmark Strategien}

Für die Beurteilung der Performance der impliziten Strategien werden passive Anlageformen und aktive Vergleichsstrategien auf Basis historischer Renditezeitreihen für die betrachteten Investoren ausgewertet. Hierzu werden als aktive Benchmarks die Matrizen $M_{2}, M_{3}$ und $M_{4}$ aus den historischen Log-Renditen geschätzt und in das Asset Allokationsproblem (5.1) eingesetzt und ausgewertet. Neben den Stichprobenschätzern werden auch die in Martellini und Ziemann (2010) eingeführten strukturierten Schätzer $\widehat{M_{k}^{C C}}$ bzw. $\widehat{M_{k}^{S F}}$ auf Basis des Constant-Correlation-Ansatzes und der Annahme eines Ein-FaktorModells untersucht. In Analogie zu Martellini und Ziemann (2010) werden als historischer Schätzzeitraum die Renditen die letzten 60 Monate gewählt.

Des Weiteren werden auch auf Shrinkage Ansätzen basierende Schätzer aus Martellini und Ziemann (2010) untersucht. $^{18}$ Für diese Ansätze werden optimale Mischungen zwischen dem Stichprobenschätzer $\widehat{M}_{k}$ der Matrix höherer Ordnung und der entsprechenden geschätzten strukturierten Matrix $\widehat{M_{k}^{C C}}$ bzw. $\widehat{M_{k}^{S F}}$ gesucht. Zur Bestimmung der optimalen Mischung dient die Frobeniusnorm ${ }^{19}$. Hierzu wird die Differenz (bzw. der Abstand) aus der Konvexkombination der Schätzer und der wahren (unbekannten Matrix) $M_{k}$ minimiert:

$$
\min _{\alpha_{k}^{C C} \in[0,1]}\left\|\alpha_{k}^{C C} \widehat{M_{k}^{C C}}+\left(1-\alpha_{k}^{C C}\right) \widehat{M_{k}}-M_{k}\right\|_{F} \quad k=2,3,4
$$

bzw.

$$
\min _{\alpha_{k}^{S F} \in[0,1]}\left\|\alpha_{k}^{S F} \widehat{M_{k}^{S F}}+\left(1-\alpha_{k}^{S F}\right) \widehat{M_{k}}-M_{k}\right\|_{F} \quad k=2,3,4 .
$$

Für die optimalen Shrinkageparameter $\alpha_{k}^{*}$ lassen sich asymptotische Eigenschaften herleiten und Schätzverfahren bestimmen. ${ }^{20}$ Tabelle 5.3 zeigt beispielhaft deskriptive Statistiken für die geschätzten Werte des optimalen Shrinkageparameters $\alpha_{k}^{C C}$ im Falle des vollstän-

Das sechste Moment wird dann gegeben durch:

$$
\begin{aligned}
& e^{r \tau} E^{Q}\left\{e^{-r \tau} H_{6}^{\prime \prime}\left(S_{t+\tau}\right)\right\}-6 e^{r \tau} E^{Q}\left\{e^{-r \tau} H_{5}^{\prime \prime}\left(S_{t+\tau}\right)\right\} \mu_{t, \tau} \\
& +15 e^{r \tau} X(t, \tau) \mu_{t, \tau}^{2}-20 e^{r \tau} W(t, \tau) \mu_{t, \tau}^{3}+15 e^{r \tau} V(t, \tau) \mu_{t, \tau}^{4}-5 \mu_{t, \tau}^{6}
\end{aligned}
$$

Für die Kovarianzmatrix $M_{2}$ sind diese Schätzer durch Ledoit und Wolf (2003) und Ledoit und Wolf (2004) entwickelt worden.

Die Frobeniusnorm einer Matrix entspricht der Summe aller quadrierten Einträge.

Die entsprechenden Formeln finden sich in Martellini und Ziemann (2010). 
digen Datensatzes $N=30$. Die erhaltenen Werte stimmen in der Tendenz mit denen aus der Studie von Martellini und Ziemann (2010) überein. Die Methodik liefert für den Shrinkageparameter der Koschiefematrix $M_{3}$ den höchsten erwarteten Wert und suggeriert, dass in diesem Fall fast ausschließlich der strukturierte Schätzer für den Shrinkage Ansatz gewählt wird. Dies ist im Einklang mit der Intuition, dass Schätzer für ungerade Momente stark streuen. ${ }^{21}$

Tabelle 5.3: Deskriptive Statistiken für die optimalen Shrinkageintensitäten für die strukturierten Matrizen $M_{2}, M_{3}$ und $M_{4}$

\begin{tabular}{lccccc}
\hline & ERW & STD & $25 \%$ & MED & $75 \%$ \\
\hline$\alpha_{2}^{C C}$ & 0,660 & 0,162 & 0,523 & 0,637 & 0,754 \\
$\alpha_{3}^{C C}$ & 0,998 & 0,015 & 1,000 & 1,000 & 1,000 \\
$\alpha_{4}^{C C}$ & 0,892 & 0,081 & 0,828 & 0,893 & 0,989 \\
\hline
\end{tabular}

Diese Tabelle zeigt deskriptive Statistiken für die optimalen Shrinkageintensitäten für die strukturierten Matrizen $M_{2}, M_{3}$ und $M_{4}$. Der Zeitraum umfasst Januar 1998 bis November 2010.

Neben den aktiven historischen Benchmark Strategien werden auch drei passive Anlageformen betrachtet: zum einen eine Investition in einen gleichgewichteten Index $(1 / N)$, zum anderen Investitionen, welche den marktkapitalisierten (Dow_MCap) und preisgewichteten (Dow) Index duplizieren. Im Fall $N=30$ entspricht der preisgewichtete Index nicht einer direkten Investition in den DJIA, da dieser kein Performance-Index ist und somit insbesondere keine Dividenden berücksichtigt. Tabelle 5.4 liefert eine Übersicht aller betrachteten Strategien, welche in der empirischen Studie für die betrachteten Investoren ausgewertet worden sind.

21 Vgl. Diskussion in Martellini und Ziemann (2010). 
Tabelle 5.4: Übersicht der betrachteten aktiven und passiven Anlagestrategien der empirischen Studie

\begin{tabular}{lc}
\hline \multicolumn{1}{c}{ Symbol } & \multicolumn{1}{c}{ Beschreibung } \\
\hline \multicolumn{1}{c}{ Passive Anlagestrategien } \\
\hline Dow & Gleichgewichteter Index \\
Dow_MCap & Preisgewichteter Index \\
\hline Aktive Anlagestrategien auf Basis historischer Renditezeitreihen \\
\hline Sample & Stichprobenschätzer \\
ConstCor & Annahme konstanter Korrelationen \\
Shrink_CC & Optimale Mischung aus Sample und ConstCor \\
SingleFactor & Annahme eines Ein-Faktor-Markt-Modells \\
Shrink_SF & Optimale Mischung aus Sample und SingleFactor \\
\hline \multicolumn{2}{c}{ Aktive Anlagestrategien auf Basis impliziter Informationen } \\
\hline Impl_CC & Constant Correlation Ansatz \\
Impl_SF & Annahme eines Ein-Faktor-Modells \\
\hline
\end{tabular}

Diese Tabelle zeigt eine Übersicht aller betrachteten aktiven und passiven Anlagestrategien der empirischen Studie.

\subsubsection{Performance-Kennzahlen}

Die Beurteilung der Performance der untersuchten Strategien aus Tabelle 5.4 erfolgt gemäß der nachfolgenden vier Metriken: Excess Return, realisierte Volatilität und Turnover sowie als zentrale Größe der Monetary Utility Gain.

(i) Excess Return $\hat{\mu}$

Die Berechnung des Excess Returns erfolgt, indem zu jedem Beobachtungszeitpunkt die realisierte out-of-sample Log-Rendite der untersuchten Strategie bestimmt und um die Rendite der entsprechenden risikofreien Anlage der Periode verringert wird. Der Mittelwert der erhaltenen Zeitreihe wird mit $\hat{\mu}$ bezeichnet.

(ii) Realisierte Volatilität $\hat{\sigma}$

Nach Bestimmung der optimalen Gewichte zu Beginn des Anlagehorizonts wird die Entwicklung des Portfolios auf Basis von Tages-Log-Renditen verfolgt. Aus dieser Wertentwicklung wird die Standardabweichung geschätzt. Diese entspricht der rea- 
lisierten Volatilität einer bestimmten Periode. Der Mittelwert aus der erhaltenen Zeitreihe entspricht der realisierten Volatilität $\hat{\sigma} .{ }^{22}$

(iii) Monetary Utility Gain $M U G$

Der Monetary Utility Gain für zwei Strategien 1 und 2 entspricht dem spezifischen Zinssatz $M U G$, bei welchem der Investor mit Nutzenfunktion $U$ indifferent zwischen dem Nutzen der realisierten Endvermögen der Strategie $1\left(1+r_{t}^{1}\right)$ und dem Nutzen aus dem mit dem Zinssatz $M U G$ aufgezinsten Endvermögen der Renditen der Strategie $2\left(1+r_{t}^{2}\right)$ ist. Speziell für den Fall eines Investors mit CRRA Nutzenfunktion mit Risikoaversion $\gamma$ berechnet sich der Monetary Utility Gain MUG der Strategien 1 und 2 durch

$$
\begin{aligned}
& \frac{1}{153} \sum_{t=1}^{153} \frac{\left(1+r_{t}^{1}\right)^{1-\gamma}-1}{1-\gamma} & = & \frac{1}{153} \sum_{t=1}^{153} \frac{\left((1+M U G) \cdot\left(1+r_{t}^{2}\right)\right)^{1-\gamma}-1}{1-\gamma} \\
\Rightarrow & & 1+M U G & =\left(\frac{\frac{1}{153} \sum_{t=1}^{153}\left(1+r_{t}^{1}\right)^{1-\gamma}}{\frac{1}{153} \sum_{t=1}^{153}\left(1+r_{t}^{2}\right)^{1-\gamma}}\right)^{\frac{1}{1-\gamma}} .
\end{aligned}
$$

Falls der Monetary Utility Gain positiv ist, kann die Strategie 1 (Benchmark) für den Investor als lohnenswert bzw. nutzenbringend gegenüber der Strategie 2 interpretiert werden. Negative Werte hingegen sind dementsprechend als unvorteilhaft bzw. nutzenschmälernd zu verstehen. Die Annualisierung der Kennzahl erfolgt durch $12 \cdot \log (1+M U G)$.

(iv) Turnover TO

Um Transaktionskosten für die Umschichtung an den Beobachtungszeitpunkten der jeweiligen Strategie zu berücksichtigen, wird das Turnover ${ }^{23}$ bestimmt. Hierzu werden an jedem Beobachtungszeitpunkt die Gewichte des "ursprünglichen" Portfolios bestimmt, jeweils mit dem "neuen" Gewicht für den Anlagezeitraum verglichen und der Absolutbetrag aus der Differenz beider gebildet. Die Summe über alle $N$ Port-

22 Die realisierte Volatilität entpricht zwar nicht der Standardabweichung der Excess Returns, kann jedoch aus Gründen der Interpretation als Vertreter dieser herangezogen werden. Die realisierte Volatilität ist der Standardabweichung der Excess Returns vorzuziehen, da sie eine genauere Schätzung der tatsächlichen Volatilität liefert und zugleich für Signifikanztests verwendet werden kann. 
foliopositionen ergibt die Umschichtung in einem Zeitpunkt. Der Mittelwert aus der erhaltenen Zeitreihe entspricht dem Turnover der Strategie: ${ }^{24}$

$$
\mathrm{TO}=\frac{1}{153} \sum_{i=1}^{153} \sum_{j=1}^{N}\left|\omega_{j, t=i+1}-\omega_{j, t^{+}=i}\right| .
$$

Des Weiteren können mit Hilfe des Turnovers auch Excess Returns und um Transaktionskosten bereinigte MUGs bestimmt werden. Hierzu wird die realisierte Rendite zusätzlich verringert, und zwar um den entsprechenden Turnover des Beobachtungszeitpunkts multipliziert mit einem vorgegebenen Transaktionskostensatz. Der Monetary Utility Gain wird dementsprechend aus den bereinigten Zeitreihen bestimmt. Als Transaktionskostensatz werden in dieser Studie 10 Basispunkte angesetzt. ${ }^{25}$

Für die nachfolgende Studie werden zur Beurteilung der Performance der aktiven historischen und der passiven Strategien die Monetary Utility Gains gegenüber der impliziten Strategien gebildet. Dies bedeutet, dass der Investor die Performance der Benchmark Strategien aus Tabelle 5.4 mit der Performance der aktiven Strategien auf Basis impliziter Informationen ins Verhältnis setzt. Ein positiver MUG bedeutet, dass die implizite Strategie lohnenswerter gegenüber dem Benchmark ist bzw. zu einem Nutzenvorteil führt.

Der Vorteil der MUGs gegenüber anderen Performancemaßen, wie der Sharpe-Ratio, welche auf Erwartungswert und Varianz basiert, ist, dass diese Kennzahl beim Vergleich zweier Strategien auch die höheren Momente der Renditeverteilung berücksichtigt. Im Rahmen dieser Studie ist dies essentiell, da die betrachteten Investoren ja gerade die höheren Momente in ihr Nutzenkalkül einbeziehen und ihre Asset Allokation auf Basis von Schiefe und Kurtosis treffen. Ein Performancemaß, welches ausschließlich aus einer $\mu-\sigma$ Nutzenfunktion abgeleitet wird, kann somit nicht das erzielte Nutzenniveau der betrachteten Strategien wiedergeben. Das Monetary Utility Gain ist eng mit dem Sicherheitsäquivalent aus Kapitel 3 verwandt. Im weiteren Sinne kann das Sicherheitsäquivalent als MUG gegenüber der sicheren Anlage interpretiert werden.

\subsection{Ergebnisse der empirischen Studie}

Bevor die Ergebnisse der empirischen Studie zur taktischen Asset Allokation präsentiert werden, werden zunächst die im vorherigen Abschnitt eingeführten Korrelationen höherer

24 + bedeutet in diesem Fall "am Ende des Anlagehorizonts". Der Turnover liegt zwischen 0 und 2.

25 Vgl. Plyakha, Uppal und Vilkov (2012), Kempf, Korn und Saßning (2012) und die darin angegebene Literatur. 
Momente auf Basis impliziter Informationen betrachtet und insbesondere mit den historisch geschätzten Werten verglichen.

\subsubsection{Korrelationen höherer Momente}

Zur Formulierung der Matrizen $M_{2}, M_{3}$ und $M_{4}$ auf Basis impliziter Informationen nach dem Constant-Correlation-Ansatz wurden im vorherigen Abschnitt die Korrelationen $\rho^{M_{2}}$, $\rho^{M_{3}}$ und $\rho^{M_{4}}$ definiert. Diese Korrelationen stellen für die Varianz, Schiefe und Kurtosis die Abhängigkeitsstruktur in den höheren Momenten dar. Sie enthalten somit wichtige Informationen über mögliche Diversifikationseffekte für die Portfolioselektion.

Abbildung 5.1 zeigt die implizit ermittelten Korrelationen über den out-of-sample Zeitraum. Des Weiteren sind zur Vergleichbarkeit auch die realisierten und historisch bestimmten Werte abgebildet. ${ }^{26}$ Die realisierten Korrelationen für Varianz und Kurtosis sowie die entsprechenden geschätzten Werte sind ausschließlich positiv. Dies ist durchaus erwartet, da die Mitglieder eines Indexes im Querschnitt eine tendenziell ähnliche Entwicklung aufweisen. Die ermittelten Korrelationen sind deutlich unter eins, womit auf bedeutende Diversifikationspotentiale unter den Assets geschlossen werden kann. Die Schiefe-Korrelation wird realisiert und historisch um null ermittelt bzw. geschätzt. Der implizite Wert hingegen ist ausschließlich negativ. Diese Beobachtung lässt sich darauf zurückführen, dass die implizite Schiefe von Index-Optionen tendenziell negativer ist als die impliziten Schiefen der individuellen Mitglieder des Indexes und verdeutlicht die Wirkung der Koschiefen auf die Index-Schiefe. ${ }^{27}$

Aus der Darstellung der realisierten Korrelation in Abbildung 5.1 für die Varianz ist der Vorteil der impliziten Identifikation gegenüber den historischen Schätzwerten unmittelbar zu erfassen. Richtung und Tendenz des realisierten und des prognostizierten Wertes stimmen überein. Zudem wird die zeitliche Struktur verbessert abgebildet. Eine Überschätzung des realisierten Wertes durch den impliziten Wert ist insbesondere im Zeitraum bis 2006 zu erkennen und lässt auf ein positives Korrelations-Risikoprämie schließen. ${ }^{28}$ Der historische Schätzer wird bis Mitte 2008 fast konstant auf einen Wert um 0,25 geschätzt. Ab Mitte 2008 wird ein Schock in Form eines Sprungs auf ein Niveau von 0,45 während der Finanzmarktkrise beobachtet. Die zeitliche Struktur des realisierten Wertes wird durch den historischen Schätzer nicht abgebildet. Dies hat zur Folge, dass die Korrelation im

26 Die realisierten Korrelationen werden aus Tagesrenditen des entsprechenden Anlagehorizonts ermittelt. Für die Schiefe und Kurtosis sind gemäß den Häufigkeiten aus Tabelle 5.1 die gewichteten Korrelationen $\bar{\rho}$ für die realisierten und historischen Werte abgebildet.

27 Vgl. Bakshi, Kapadia und Madan (2003).

28 Vgl. Driessen, Maenhout und Vilkov (2009) und DeMiguel, Plyakha, Uppal und Vilkov (2012). 

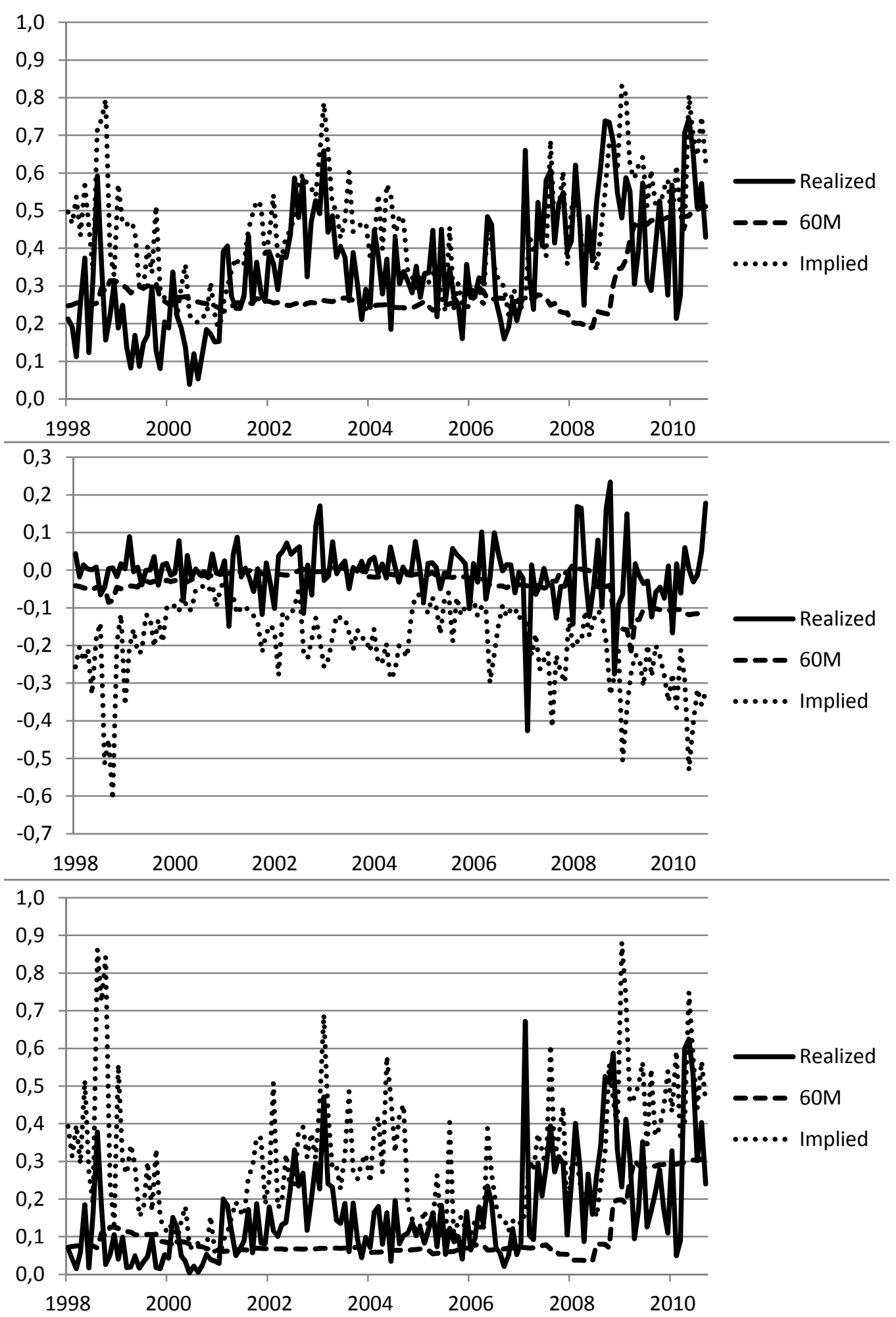

Abbildung 5.1: Diese Abbildung zeigt die realisierte, historisch (60M) und implizit geschätzte Korrelation $\rho^{M_{2}}$ sowie die gemäß den Häufigkeiten aus Tabelle 5.1 gewichteten Korrelationen $\overline{\rho^{M_{3}}}$ und $\overline{\rho^{M_{4}}}$. Der Zeitraum beträgt Februar 1998 bis November 2010. 
Zeitablauf teilweise über- und unterschätzt wird. Eine Betrachtung des realisierten Wertes zeigt einen antizyklischen Verlauf der Korrelation, womit insbesondere in Krisenzeiten auf ein geringeres Diversifikationspotential zu schließen ist.

Ein vergleichbares Ergebnis zeigt die Abbildung für die Kurtosis-Korrelation. Die zeitliche Struktur des realisierten Wertes wird im Vergleich zwischen historischen und impliziten Verfahren ausschließlich durch den impliziten Wert abgebildet. Jedoch lässt sich eine starke Verzerrung, in Form einer deutlichen Überschätzung des realisierten Wertes, erkennen. Erneut wird ein antizyklischer Verlauf der Korrelation beobachtet. Ein Vergleich zwischen den realisierten Werten der Varianz- und Kurtosis-Korrelation zeigt eine vergleichbare zeitliche Struktur auf.

Für die implizite Schiefe-Korrelation hingegen wird eine deutliche Unterschätzung des realisierten Wertes im Gegensatz zum historischen Schätzwert, welcher keine systematische Verzerrung aufzeigt, festgestellt. Die zeitliche Struktur des realisierten Wertes ist weder prozyklisch noch antizyklisch und kann durch keinen der beiden betrachteten Schätzmethoden abgebildet werden.

Zur weiteren Beurteilung der Prognose der realisierten Korrelationen aus Tabelle 5.1 durch die implizite und historische Methodik wird folgendes Regressionsmodell betrachtet:

$$
\text { Realized }=\alpha+\beta \cdot \text { Predicted }
$$

Das adjustierte Bestimmheitsmaß des Modells zeigt die Erklärung der zeitlichen Struktur des realisierten Wertes. Zur Beurteilung der Verzerrung wird die mittlere Differenz aus realisiertem und prognostiziertem Wert bestimmt. Der durchschnittliche absolute Abstand dient als weiteres Maß zur Beurteilung der Prognosqualität. Tabelle 5.5 fasst die Resultate zusammen. 
Tabelle 5.5: Mittlere Differenz und absoluter Fehler aus der Differenz der realisierten Korrelationen und den geschätzten Werten sowie das Ergebnis des Regressionsmodells

\begin{tabular}{ccccccccc}
\hline & ERW & MAE & ERW & MAE & Adj. $R^{2}$ & ERW & MAE & Adj. $R^{2}$ \\
\hline & \multicolumn{1}{c}{ Konstant Null } & \multicolumn{3}{c}{ Historisch $60 \mathrm{M}$} & \multicolumn{3}{c}{ Implizit } \\
\hline$\rho^{M_{2}}$ & 0,348 & 0,348 & 0,061 & 0,135 & $3,41 \%$ & $-0,094$ & 0,134 & $28,96 \%$ \\
& & & & & & & & \\
$\rho_{1}^{M_{3}}$ & $-0,001$ & 0,054 & 0,050 & 0,077 & $1,17 \%$ & 0,185 & 0,190 & $-0,64 \%$ \\
$\rho_{2}^{M_{3}}$ & $-0,006$ & 0,050 & 0,029 & 0,060 & $2,35 \%$ & 0,180 & 0,186 & $-0,62 \%$ \\
$\rho^{M_{3}}$ & $-0,005$ & 0,050 & 0,031 & 0,062 & $2,25 \%$ & 0,181 & 0,186 & $-0,62 \%$ \\
& & & & & & & & \\
$\rho_{1}^{M_{4}}$ & 0,280 & 0,280 & 0,072 & 0,121 & $5,56 \%$ & $-0,021$ & 0,122 & $21,01 \%$ \\
$\rho_{2}^{M_{4}}$ & 0,450 & 0,450 & 0,056 & 0,084 & $4,49 \%$ & 0,149 & 0,189 & $16,33 \%$ \\
$\rho_{3}^{M_{4}}$ & 0,216 & 0,216 & 0,064 & 0,116 & $7,64 \%$ & $-0,085$ & 0,135 & $19,78 \%$ \\
$\rho_{4}^{M_{4}}$ & 0,151 & 0,151 & 0,089 & 0,115 & $10,76 \%$ & $-0,150$ & 0,172 & $19,93 \%$ \\
$\rho^{M_{4}}$ & 0,164 & 0,164 & 0,061 & 0,105 & $9,28 \%$ & $-0,137$ & 0,162 & $19,96 \%$ \\
\hline
\end{tabular}

Diese Tabelle zeigt für die Korrelationen $\rho^{M_{2}}, \rho_{1}^{M_{3}}, \rho_{2}^{M_{3}}, \rho_{1}^{M_{4}}, \rho_{2}^{M_{4}}, \rho_{3}^{M_{4}}$ und $\rho_{4}^{M_{4}}$ sowie die gemäß den Häufigkeiten aus Tabelle 5.1 gewichteten Korrelationen $\overline{\rho^{M_{3}}}$ und $\overline{\rho^{M_{4}}}$ die mittlere Differenz (ERW) aus dem realisierten Wert und dem historisch geschätzten Wert (60M), dem implizit geschätzten Wert sowie für die Vorhersage von Null. MAE gibt den mittleren absoluten Fehler zwischen dem geschätzten und dem realisierten Wert an. Adj. $R^{2}$ zeigt das adjustierte Bestimmtheitsmaßs des Regressionsmodells Realized = $\alpha+\beta \cdot$ Predicted. Der out-of-sample Zeitraum beträgt Februar 1998 bis November 2010.

Für die Korrelation $\rho^{M_{2}}$ der Kovarianzmatrix ist an den deskriptiven Kennzahlen zu erkennen, dass der historische Schätzwert und der implizite Wert eine annähernd gleiche Verzerrung im Sinne des mittleren absoluten Abstandes aufweisen. Die mittlere Differenz zeigt weiter, dass der historische Schätzwert den realisierten Wert unterschätzt, der implizite ihn hingegen überschätzt. Dies ist im Einklang mit Driessen, Maenhout und Vilkov (2009) und deutet auf eine positive Korrelations-Risikoprämie hin. Das adjustierte Bestimmtheitsmaßs bestätigt die Beobachtung aus der Abbildung 5.1, dass die zeitliche Struktur des realisierten Wertes ausschließlich durch den impliziten Wert wiedergegeben wird.

Die Schiefe-Korrelationen $\rho_{1}^{M_{3}}$ und $\rho_{2}^{M_{3}}$ können weder durch die historische noch durch die implizite Methodik zufriedenstellend prognostiziert werden. Das adjustierte Bestimmtheitsmaß weist sogar für den impliziten Schätzer negative Werte auf. Die deskriptiven Statistiken zeigen für den impliziten Wert, wie in Abbildung 5.1 für die Schiefe beobachtet, eine deutliche Unterschätzung des realisierten Wertes. Die Verzerrung des historischen Wertes ist zwar deutlich geringer als die des impliziten, jedoch liefert dieser im Vergleich zu einem konstanten Null Schätzwert kein überzeugendes Verfahren. Zusammenfassend wird durch keine der betrachteten Schätzer der realisierte Wert zufriedenstellend prognostiziert. 
Abschließend sei die Prognose für die Korrelationen $\rho_{1}^{M_{4}}, \rho_{2}^{M_{4}}, \rho_{3}^{M_{4}}$ und $\rho_{4}^{M_{4}}$ der Kokurtosismatrix betrachtet. Die deskriptiven Ergebnisse zeigen, wie schon für die Varianz gesehen, eine deutliche Überschätzung, mit Ausnahme der Korrelation $\rho_{2}^{M_{4}}$, durch den impliziten Schätzer. Der entsprechende historische Wert weist eine Unterschätzung auf. Der historische Schätzer zeigt weiter eine geringere Verzerrung, gemessen am mittleren absoluten Fehler, gegenüber dem impliziten Wert. Die zeitliche Struktur wird wiederum von dem impliziten Schätzer zufriedenstellender wiedergegeben. Zusammenfassend liefert der historische Schätzer eine geringere Verzerrung der realisierten Werte, der implizite jedoch die bessere Prognose der zeitlichen Struktur.

Die realisierten Korrelationen für Schiefe und Kurtosis werden mit Hilfe von vierten und sechsten Momenten definiert. Inwieweit eine stabile Schätzung dieser Größen aus Tageskursen eines Monats möglich ist, ist nicht Bestandteil dieser Untersuchung und sollte kritisch hinterfragt. Da die Korrelationen im Gegensatz zu zentralen Momenten normierte Größen sind, ist die Schätzung der Parameter jedoch weniger problematisch einzustufen als die Bestimmung der realisierten Werte aus Kapitel 4.

Von weiterem Interesse sind die Abhängigkeiten der impliziten Korrelationen untereinander. Aus der Abbildung 5.1 ist zu beobachten, dass zwischen der impliziten VarianzKorrelation und Kurtosis-Korrelation eine vergleichbare zeitliche Struktur besteht. Genauer ergibt sich eine Korrelation von 0,9412 zwischen den Zeitreihen und eine lineare Regressionsanalyse liefert ein adjustiertes Bestimmtheitsmaß von 88,53\%. Für die implizite Schiefe-Korrelation besteht eine ähnliche Abhängigkeit zu der impliziten VarianzKorrelation, jedoch eine stark negative. Konkret ist die Korrelation -0,8571 und das adjustierte Bestimmtheitsmaß 73,28\%. Dies zeigt eine hohe Abhängigkeit unter den impliziten Korrelation höherer Ordnung.

Abbildung 5.2 zeigt die Zeitreihen der Differenzen aus der impliziten Schiefe-Korrelation und der impliziten Varianz-Korrelation sowie die Differenzen aus der impliziten Kurtosisund Varianz-Korrelation. Für die letztere Differernz ist eine fast konstante Zeitreihe ermittelt worden. Dies impliziert, dass sich die Abhängigkeitsstrukturen für Varianz und Kurtosis im Zeitablauf konstant bewegen und auf neue Marktsituation parallel reagieren. Für die Schiefe hingegen wird ein antizyklischer Verlauf beobachtet.

Albuquerque (2012) stellt in diesem Zusammenhang auf der Grundlage von modelltheoretischen Überlegegungen fest, dass die Koschiefe in einem Marktportfolio als Maß für die Sensitivität von Volatilitäten und Korrelationen der übrigen Mitglieder bei einem Schock eines individuellen Mitglieds, in Form eines starken Kursrückganges, zu interpretieren ist. Diese Anschauung kann herangezogen werden, um den antizyklischen Verlauf in den groben Ansätzen zu verstehen. In ruhigen Marktphasen wird der Einfluss von einem individuellen Schock auf die Volatilitäten und Korrelationen der übrigen Mitglieder somit 


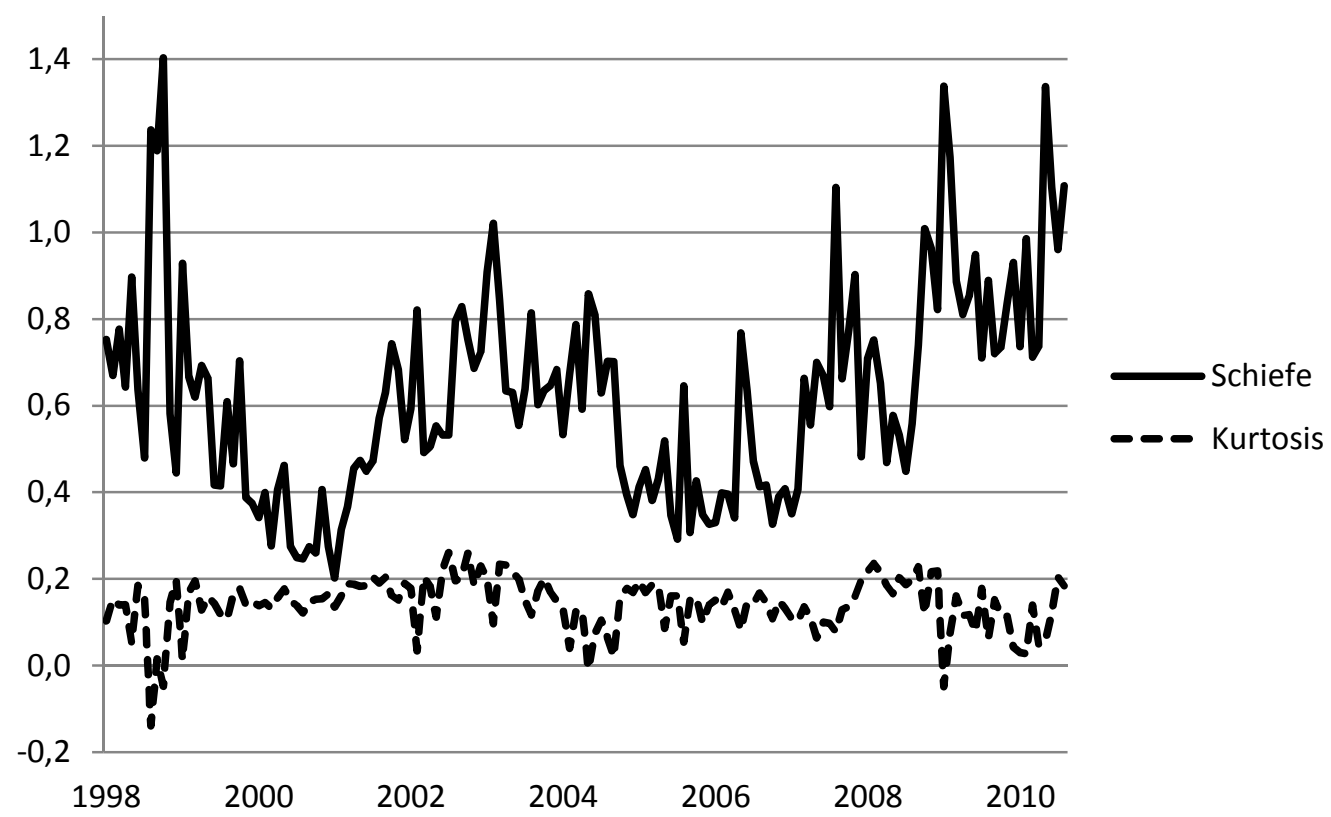

Abbildung 5.2: Diese Abbildung zeigt die Differenzen aus der implizit geschätzten Korrelation $\rho^{M_{2}}$ und der impliziten Korrelation $\rho^{M_{3}}$ (Schiefe) sowie der impliziten Korrelation $\rho^{M_{4}}$ (Kurtosis). Der Zeitraum beträgt Februar 1998 bis November 2010.

geringer eingeschätzt als in Phasen eines unruhigen bzw. volatilen Marktumfeldes. Im weiteren Sinne kann die Differenz somit als Grad der "Nervosität des Marktes" interpretiert werden.

Zusammenfassend ist festzuhalten, dass die impliziten Korrelation deutlich miteinander korrelieren und voneinander abhängen. Die Kurtosis-Korrelation unterscheidet sich fast auschließlich durch einen konstanten Term von der Varianz-Korrelation. Für die Schiefe hingegen wird eine antizyklische Beziehung ermittelt.

\subsubsection{Ergebnisse der Portfoliostudie}

Zur Untersuchung der zentralen Forschungsfrage zum Nutzen von impliziten Informationen in der Asset Allokation werden folgend die Ergebnisse der Portfoliostudie differenziert nach dem Constant-Correlation-Ansatz bzw. der Annahme eines Ein-Faktor-Modells für die Strategien aus Tabelle 5.4 vorgestellt. Die Diskussion konzentriert sich auf den Constant-Correlation-Ansatz. Zur Untersuchung der Robustheit werden die PerformanceKennzahlen auch bei Berücksichtigung von Transaktionskosten bestimmt. Die Resultate im Falle der Annahme eines Ein-Faktor-Modells werden aufgrund der Ähnlichkeit zu den Ergebnissen aus dem Constant-Correlation-Ansatz nur ausgewählt vorgestellt und dienen als weiterer Robustheitstest für die Interpretation und Diskussion. 
Tabelle 5.6: MUGs der passiven und historischen Strategien gegenüber der impliziten Strategie auf Basis des Constant-Correlation-Ansatzes

\begin{tabular}{lcccccccc}
\hline & \multicolumn{3}{c}{$\mathrm{RRA}=2$} & \multicolumn{2}{c}{$\mathrm{RRA}=4$} & \multicolumn{2}{c}{$\mathrm{RRA}=6$} & \multicolumn{2}{c}{$\mathrm{RRA}=8$} \\
\hline \multicolumn{1}{c}{$\mathrm{N}=10$} & & & & & & & & \\
\hline 1/N & $3,62 \%$ & $(0,09)$ & $4,79 \%$ & $(0,05)$ & $5,93 \%$ & $(0,03)$ & $6,46 \%$ & $(0,04)$ \\
Dow & $2,76 \%$ & $(0,15)$ & $3,60 \%$ & $(0,10)$ & $4,38 \%$ & $(0,08)$ & $4,54 \%$ & $(0,09)$ \\
Dow_MCap & $4,38 \%$ & $(0,06)$ & $5,68 \%$ & $(0,03)$ & $6,94 \%$ & $(0,01)$ & $7,59 \%$ & $(0,02)$ \\
Sample & $-0,08 \%$ & $(0,51)$ & $0,46 \%$ & $(0,41)$ & $1,05 \%$ & $(0,30)$ & $1,14 \%$ & $(0,31)$ \\
ConstCor & $1,03 \%$ & $(0,28)$ & $1,71 \%$ & $(0,18)$ & $2,42 \%$ & $(0,10)$ & $2,65 \%$ & $(0,10)$ \\
Shrink_CC & $0,24 \%$ & $(0,44)$ & $0,79 \%$ & $(0,33)$ & $1,38 \%$ & $(0,23)$ & $1,51 \%$ & $(0,23)$ \\
\hline \multicolumn{1}{c}{$\mathrm{N}=20$} & & & & & & & & \\
\hline 1/N & $2,78 \%$ & $(0,22)$ & $4,01 \%$ & $(0,15)$ & $5,50 \%$ & $(0,09)$ & $7,06 \%$ & $(0,06)$ \\
Dow & $2,38 \%$ & $(0,23)$ & $3,04 \%$ & $(0,19)$ & $3,88 \%$ & $(0,15)$ & $4,71 \%$ & $(0,12)$ \\
Dow_MCap & $4,44 \%$ & $(0,11)$ & $5,50 \%$ & $(0,07)$ & $6,74 \%$ & $(0,04)$ & $7,97 \%$ & $(0,03)$ \\
Sample & $0,80 \%$ & $(0,36)$ & $0,88 \%$ & $(0,35)$ & $1,15 \%$ & $(0,33)$ & $1,46 \%$ & $(0,29)$ \\
ConstCor & $2,17 \%$ & $(0,14)$ & $2,61 \%$ & $(0,12)$ & $3,23 \%$ & $(0,07)$ & $3,90 \%$ & $(0,05)$ \\
Shrink_CC & $1,13 \%$ & $(0,29)$ & $1,34 \%$ & $(0,27)$ & $1,75 \%$ & $(0,22)$ & $2,19 \%$ & $(0,18)$ \\
\hline \multicolumn{1}{c}{$\mathrm{N}=30$} & & & & & & & & \\
\hline 1/N & $1,41 \%$ & $(0,36)$ & $3,40 \%$ & $(0,20)$ & $5,65 \%$ & $(0,10)$ & $7,45 \%$ & $(0,06)$ \\
Dow & $1,02 \%$ & $(0,38)$ & $2,21 \%$ & $(0,26)$ & $3,59 \%$ & $(0,18)$ & $4,43 \%$ & $(0,14)$ \\
Dow_MCap & $3,23 \%$ & $(0,17)$ & $4,45 \%$ & $(0,10)$ & $5,77 \%$ & $(0,06)$ & $6,46 \%$ & $(0,05)$ \\
Sample & $1,41 \%$ & $(0,28)$ & $1,58 \%$ & $(0,26)$ & $1,81 \%$ & $(0,24)$ & $1,38 \%$ & $(0,31)$ \\
ConstCor & $1,87 \%$ & $(0,18)$ & $2,48 \%$ & $(0,12)$ & $3,14 \%$ & $(0,07)$ & $3,27 \%$ & $(0,08)$ \\
Shrink_CC & $1,41 \%$ & $(0,24)$ & $1,78 \%$ & $(0,19)$ & $2,21 \%$ & $(0,14)$ & $2,04 \%$ & $(0,19)$ \\
\hline
\end{tabular}

Diese Tabelle zeigt die annualisierten MUGs der passiven und historischen Strategien gegenüber der impliziten Strategie auf Basis des Constant-Correlation-Ansatzes für Investoren mit CRRA Nutzenfunktionen. In Klammern sind aus einem Bootstrap-Verfahren mit 10.000 Simulationen abgeleitete p-Werte auf die Hypothese angegeben, dass der entsprechende MUG größer null ist. Der out-of-sample Zeitraum beträgt Februar 1998 bis November 2010.

Tabelle 5.6 zeigt die Monetary Utility Gains für die betrachteten Strategien und Anlageuniversen gegenüber der impliziten Strategie Impl_CC. Über alle Anlageuniversen und Risikoaversionen zeigt sich, dass die implizite Strategie den passiven Benchmarks und aktiven Vergleichsstrategien vorzuziehen ist. Der marktkapitalisierte (passive) Benchmark Dow_MCap wird für fast alle betrachteten Fälle sogar durch die implizite Strategie auf einem 10\%-Niveau statistisch signifikant geschlagen. Lediglich im Falle des Anlageuniversums $N=10$ für die Risikoaversion 2 liefert der Stichprobenschätzer einen marginal negativen MUG und ist somit als nutzenbringender gegenüber dem impliziten Ansatz einzuordnen.

Für die passiven Benchmark Strategien ist über alle Anlageuniversen für die betrachteten Investoren zu beobachten, dass der erzielte MUG durch die implizite Strategie umso höher ist, je risikoaverser der Investor ist. Dies lässt vermuten, dass die implizite Strategie ins- 
besondere im Bezug auf die höheren Momente überzeugt, da risikoaverse Investoren diese stärker in ihrem Nutzenkalkül einbeziehen. Diese Vermutung wird zusätzlich dadurch unterstützt, dass für die Investoren mit Risikoaversion 6 und 8 die MUGs auch im Vergleich zum 1/N Benchmark auf einem 10\%-Niveau statistisch signifikant sind.

In der Betrachtung der MUGs für die historischen Vergleichsstrategien lässt sich die Beobachtung, dass die implizite Strategie mit steigender Risikoaffinität der Investoren vorteilhafter wird, robust über alle Anlageuniversen bestätigen. Unter den betrachteten historischen Strategien liefert der Stichprobenschätzer den geringsten Nutzenverlust gegenüber der impliziten Strategie. Der Shrinkage Ansatz ist wiederum dem vollständig strukturierten Schätzer vorzuziehen, welcher für risikoaverse Investoren statistisch signifikant geschlagen wird.

Das Resultat bezüglich der Reihenfolge der Vorteilhaftigkeit der historischen Strategien steht im Widerspruch zu den Ergebnissen aus Martellini und Ziemann (2010), bei welchen gerade ein Vorteil der strukturierten Schätzer gegenüber dem Stichprobenschätzer festgestellt wird. Eine mögliche Erklärung für dieses Resultat ist, dass in dieser Studie im Gegensatz zu Martellini und Ziemann (2010) eine strikte Leerverkaufsbeschränkung gefordert wird. Restriktionen der Portfoliogewichte haben strukturierende Wirkung auf die entsprechenden Schätzer und diese Strukturierung dominiert diejenige durch die Annahme der konstanten Korrelation. ${ }^{29}$ Jagannathan und Ma (2003) zeigen in diesem Zusammenhang für das Minimum-Varianz-Portfolio, dass bei Annahme einer konstanten Korrelation bzw. eines entsprechenden Shrinkage Ansatzes Portfoliorestriktionen die Performance sogar "leicht" verschlechtern.

Zur weiteren Beurteilung und Überprüfung der Beobachtung aus den MUGs zeigt Tabelle 5.7 Excess Returns und realisierte Volatilitäten der betrachteten Strategien. ${ }^{30}$

29 Vgl. Jagannathan und Ma (2003) und DeMiguel, Garlappi, Nogales und Uppal (2009a).

30 Zur Übersichtlichkeit werden für die Performancemaße Excess Return und realisierte Volatilität die Ergebnisse nur für die Risikoaversionen 4 und 8 wiedergegeben. 
Tabelle 5.7: Excess Returns und realisierte Volatilitäten der passiven, historischen und impliziten Strategien auf Basis des Constant-Correlation-Ansatzes

\begin{tabular}{|c|c|c|c|c|c|c|c|c|}
\hline & \multicolumn{2}{|c|}{$\hat{\mu}$} & \multicolumn{2}{|c|}{$\hat{\sigma}$} & \multicolumn{2}{|c|}{$\hat{\mu}$} & \multicolumn{2}{|c|}{$\hat{\sigma}$} \\
\hline $\mathrm{N}=10$ & \multicolumn{4}{|c|}{$\mathrm{RRA}=4$} & \multicolumn{4}{|c|}{$\mathrm{RRA}=8$} \\
\hline $1 / \mathrm{N}$ & $-1,87 \%$ & $(0,27)$ & $20,93 \%$ & $(0,00)$ & $-1,87 \%$ & $(0,37)$ & $20,93 \%$ & $(0,00)$ \\
\hline Dow & $-1,16 \%$ & $(0,38)$ & $20,06 \%$ & $(0,00)$ & $-1,16 \%$ & $(0,50)$ & $20,06 \%$ & $(0,00)$ \\
\hline Dow_MCap & $-2,57 \%$ & $(0,19)$ & $21,46 \%$ & $(0,00)$ & $-2,57 \%$ & $(0,28)$ & $21,46 \%$ & $(0,00)$ \\
\hline Sample & $1,51 \%$ & $(0,90)$ & $18,89 \%$ & $(0,00)$ & $1,52 \%$ & $(0,77)$ & $18,90 \%$ & $(0,00)$ \\
\hline ConstCor & $0,47 \%$ & $(0,68)$ & $18,41 \%$ & $(0,00)$ & $0,47 \%$ & $(0,83)$ & $18,43 \%$ & $(0,00)$ \\
\hline Shrink_CC & $1,22 \%$ & $(0,98)$ & $18,42 \%$ & $(0,00)$ & $1,27 \%$ & $(0,85)$ & $18,44 \%$ & $(0,00)$ \\
\hline Impl_ēC & $1,26 \%$ & & $17,07 \%$ & & $0,90 \%$ & & $17,34 \%$ & \\
\hline $\mathrm{N}=20$ & \multicolumn{4}{|c|}{$\mathrm{RRA}=4$} & \multicolumn{4}{|c|}{$\mathrm{RRA}=8$} \\
\hline $1 / \mathrm{N}$ & $0,49 \%$ & $(0,59)$ & $21,25 \%$ & $(0,00)$ & $0,49 \%$ & $(0,55)$ & $21,25 \%$ & $(0,00)$ \\
\hline Dow & $0,63 \%$ & $(0,59)$ & $19,82 \%$ & $(0,00)$ & $0,63 \%$ & $(0,55)$ & $19,82 \%$ & $(0,00)$ \\
\hline Dow_MCap & $-1,24 \%$ & $(0,31)$ & $21,21 \%$ & $(0,00)$ & $-1,24 \%$ & $(0,29)$ & $21,21 \%$ & $(0,00)$ \\
\hline Sample & $1,94 \%$ & $(0,84)$ & $18,01 \%$ & $(0,00)$ & $1,95 \%$ & $(0,77)$ & $18,05 \%$ & $(0,00)$ \\
\hline ConstCor & $0,75 \%$ & $(0,44)$ & $17,88 \%$ & $(0,00)$ & $0,72 \%$ & $(0,39)$ & $17,90 \%$ & $(0,00)$ \\
\hline Shrink_CC & $1,69 \%$ & $(0,73)$ & $17,70 \%$ & $(0,00)$ & $1,68 \%$ & $(0,66)$ & $17,73 \%$ & $(0,00)$ \\
\hline Impl_ēC & $2,44 \%$ & & $16,72 \%$ & & $2,67 \%$ & & $16,97 \%$ & \\
\hline $\mathrm{N}=30$ & \multicolumn{4}{|c|}{$\mathrm{RRA}=4$} & \multicolumn{4}{|c|}{$\mathrm{RRA}=8$} \\
\hline $1 / \mathrm{N}$ & $1,40 \%$ & $(0,97)$ & $22,24 \%$ & $(0,00)$ & $1,40 \%$ & $(0,93)$ & $22,24 \%$ & $(0,00)$ \\
\hline Dow & $1,42 \%$ & $(0,97)$ & $20,29 \%$ & $(0,00)$ & $1,42 \%$ & $(0,91)$ & $20,29 \%$ & $(0,00)$ \\
\hline Dow_MCap & $-0,76 \%$ & $(0,51)$ & $21,23 \%$ & $(0,00)$ & $-0,76 \%$ & $(0,61)$ & $21,23 \%$ & $(0,00)$ \\
\hline Sample & $0,63 \%$ & $(0,72)$ & $17,67 \%$ & $(0,00)$ & $0,65 \%$ & $(0,88)$ & $17,68 \%$ & $(0,00)$ \\
\hline ConstCor & $0,29 \%$ & $(0,57)$ & $17,70 \%$ & $(0,00)$ & $0,28 \%$ & $(0,74)$ & $17,72 \%$ & $(0,00)$ \\
\hline Shrink_CC & $0,61 \%$ & $(0,67)$ & $17,41 \%$ & $(0,00)$ & $0,61 \%$ & $(0,85)$ & $17,45 \%$ & $(0,00)$ \\
\hline Impl_ $\bar{C} C$ & $1,54 \%$ & & $16,43 \%$ & & $1,04 \%$ & & $16,79 \%$ & \\
\hline
\end{tabular}

Diese Tabelle zeigt die Excess Returns und realisierten Volatilitäten der passiven, historischen und impliziten Strategien auf Basis des Constant-Correlation-Ansatzes für Investoren mit CRRA Nutzenfunktionen. Alle Werte sind annualisiert. In Klammern sind p-Werte von t-Tests auf die Hypothese angegeben, dass die implizite Strategie denselben Excess Return bzw. dieselbe realisierte Volatilität besitzt. Der out-ofsample Zeitraum beträgt Februar 1998 bis November 2010.

Für das Anlageuniversum $N=10$ liefert die historische Strategie auf Basis des Stichprobenschätzers für alle betrachteten Investoren den höchsten Excess Return. Die implizite Strategie erzielt den zweit- bzw. dritthöchsten Wert, jedoch sind die Werte statistisch nicht signifikant verschieden. Der positive MUG für die Risikoaversion 2 kann somit auf den höheren Excess Return der Sample Strategie zurückgeführt werden. Die weiteren historischen Strategien erzielen niedrigere Werte als der implizite Ansatz, die passiven Anlageformen sogar negative. Im Sinne der statistischen Signifikanz kann die implizite Strategie die betrachteten Benchmarks jedoch nicht schlagen. Eine Betrachtung der realisierten Volatilität kann zur Erklärung für die Resultate aus den MUGs herangezogen 
werden. Die implizite Strategie erzielt, im Einklang mit der bestehenden Literatur ${ }^{31}$, eine auf einem 1\%-Niveau statistisch signifikant geringere Volatilität. Dies gilt für alle betrachteten Investoren.

Für die weiteren Anlageuniversen $N=20$ und $N=30$ erzielt die implizite Strategie den höchsten Excess Return im Vergleich zu den aktiven Benchmark-Strategien. Gegenüber den passiven Strategien überzeugt, insbesondere für das $N=30$ Universum, der $1 / \mathrm{N}$ und Dow Benchmark. Die Renditen sind jedoch gegenüber den Bechmarks nicht statistisch signifikant verschieden. Der gleich- und preisgewichtete Index liefert in diesen Fällen auch positive Excess Returns und schlägt für den Anlagehorizont $N=30$ die historischen Vergleichsstrategien bzgl. dieser Metrik. Dies kommt auch in den MUGs aus Tabelle 5.6 zum Ausdruck, bei welchen für die Risikoaversion 2 der preisgewichtete Index (Dow) den kleinsten positiven Wert liefert. Unter den historischen Strategien liefert der Stichprobenschätzer erneut den höchsten Wert. Die Resultate bzgl. der realisierten Volatilität bestätigen sich für die weiteren Anlageuniversen. Im Vergleich zu den Benchmarks werden robust über alle Risikoaversionen die statistisch signifikant geringsten Werte erzielt.

Im Speziellen zeigt der Vergleich unter den verschiedenen Risikoaversionen für Excess Return und realisierter Volatilität für die implizite Strategie den Einfluss aus der Verwendung höherer Momente. Die Resultate deuten daraufhin, dass die Risikoaversion des Investors im Gegensatz zu dem von der Risikoaversion unabhängigen Minimum-Varianz-Portfolio einen bedeutenden Einfluss auf die out-of-sample Ergebnisse hat.

31 Vgl. z. B. Kapitel 3, Kempf, Korn und Saßnning (2012) und DeMiguel, Plyakha, Uppal und Vilkov (2012). 
Tabelle 5.8: MUGs der passiven und historischen Strategien gegenüber der impliziten Strategie auf Basis der Annahme eines Ein-Faktor-Modells

\begin{tabular}{lcccccccc}
\hline & \multicolumn{3}{c}{$\mathrm{RRA}=2$} & \multicolumn{2}{c}{$\mathrm{RRA}=4$} & \multicolumn{2}{c}{$\mathrm{RRA}=6$} & \multicolumn{2}{c}{$\mathrm{RRA}=8$} \\
\hline \multicolumn{1}{c}{$\mathrm{N}=10$} & & & & & & & & \\
\hline 1/N & $3,68 \%$ & $(0,10)$ & $4,75 \%$ & $(0,05)$ & $5,70 \%$ & $(0,04)$ & $6,41 \%$ & $(0,04)$ \\
Dow & $2,82 \%$ & $(0,16)$ & $3,56 \%$ & $(0,11)$ & $4,16 \%$ & $(0,09)$ & $4,49 \%$ & $(0,09)$ \\
Dow_MCap & $4,45 \%$ & $(0,07)$ & $5,64 \%$ & $(0,03)$ & $6,71 \%$ & $(0,02)$ & $7,54 \%$ & $(0,02)$ \\
Sample & $-0,01 \%$ & $(0,50)$ & $0,42 \%$ & $(0,40)$ & $0,82 \%$ & $(0,34)$ & $1,09 \%$ & $(0,30)$ \\
SingleFactor & $1,68 \%$ & $(0,18)$ & $2,12 \%$ & $(0,13)$ & $2,50 \%$ & $(0,10)$ & $2,76 \%$ & $(0,10)$ \\
Shrink_SF & $0,64 \%$ & $(0,37)$ & $1,05 \%$ & $(0,30)$ & $1,42 \%$ & $(0,23)$ & $1,70 \%$ & $(0,20)$ \\
\hline \multicolumn{1}{c}{$\mathrm{N}=20$} & & & & & & & & \\
\hline 1/N & $2,73 \%$ & $(0,23)$ & $4,10 \%$ & $(0,15)$ & $5,56 \%$ & $(0,09)$ & $7,12 \%$ & $(0,05)$ \\
Dow & $2,33 \%$ & $(0,24)$ & $3,12 \%$ & $(0,19)$ & $3,94 \%$ & $(0,14)$ & $4,77 \%$ & $(0,11)$ \\
Dow_MCap & $4,39 \%$ & $(0,11)$ & $5,58 \%$ & $(0,07)$ & $6,80 \%$ & $(0,04)$ & $8,03 \%$ & $(0,03)$ \\
Sample & $0,75 \%$ & $(0,37)$ & $0,96 \%$ & $(0,35)$ & $1,21 \%$ & $(0,31)$ & $1,52 \%$ & $(0,28)$ \\
SingleFactor & $1,90 \%$ & $(0,19)$ & $2,24 \%$ & $(0,16)$ & $2,61 \%$ & $(0,13)$ & $3,01 \%$ & $(0,11)$ \\
Shrink_SF & $1,27 \%$ & $(0,28)$ & $1,51 \%$ & $(0,25)$ & $1,78 \%$ & $(0,22)$ & $2,06 \%$ & $(0,19)$ \\
\hline \multicolumn{1}{c}{ N=30 } & & & & & & & & \\
\hline 1/N & $1,36 \%$ & $(0,36)$ & $3,48 \%$ & $(0,21)$ & $5,74 \%$ & $(0,10)$ & $7,90 \%$ & $(0,05)$ \\
Dow & $0,96 \%$ & $(0,38)$ & $2,29 \%$ & $(0,25)$ & $3,68 \%$ & $(0,17)$ & $4,88 \%$ & $(0,12)$ \\
Dow_MCap & $3,18 \%$ & $(0,18)$ & $4,53 \%$ & $(0,10)$ & $5,86 \%$ & $(0,06)$ & $6,91 \%$ & $(0,04)$ \\
Sample & $1,36 \%$ & $(0,29)$ & $1,65 \%$ & $(0,25)$ & $1,90 \%$ & $(0,23)$ & $1,83 \%$ & $(0,25)$ \\
SingleFactor & $1,18 \%$ & $(0,30)$ & $1,58 \%$ & $(0,24)$ & $1,93 \%$ & $(0,20)$ & $2,00 \%$ & $(0,19)$ \\
Shrink_SF & $1,10 \%$ & $(0,31)$ & $1,41 \%$ & $(0,27)$ & $1,66 \%$ & $(0,24)$ & $1,60 \%$ & $(0,25)$ \\
\hline
\end{tabular}

Diese Tabelle zeigt die annualisierten MUGs der passiven und historischen Strategien gegenüber der impliziten Strategie auf Basis der Annahme eines Ein-Faktor-Modells für Investoren mit CRRA Nutzenfunktionen. In Klammern sind aus einem Bootstrap-Verfahren mit 10.000 Simulationen abgeleitete p-Werte auf die Hypothese angegeben, dass der entsprechende MUG größer null ist. Der out-of-sample Zeitraum beträgt Februar 1998 bis November 2010.

Tabelle 5.8 zeigt die Ergebnisse für die Annahme eines Ein-Faktor-Modells. Die historischen Strategien werden nun entsprechend dieser Annahme gebildet. Die Vorteilhaftigkeit der impliziten Strategie Impl_SF gegenüber den Benchmarks wird für alle Investoren und über alle Anlageuniversen bestätigt.

Im Gegensatz zu den vorherigen Ergebnissen zeigt sich für die historischen Strategien, dass für das vollständige Anlageuniversum $N=30$ und die Risikoaversionen 2 und 4 der Stichprobenschätzer (Sample) den höchsten positiven MUG aufweist, wodurch geschlossen werden kann, dass, im Einklang mit Martellini und Ziemann (2010), die strukturierten Schätzer (SingleFactor und Shrink_SF) der Sample-Strategie vorzuziehen sind. 


\subsubsection{Berücksichtigung von Transaktionskosten}

Zur weiteren Überprüfung der Robustheit der Ergebnisse werden die MUGs bereinigt um Transaktionskosten gebildet. Tabelle 5.9 zeigt die Werte für den Constant-CorrelationAnsatz, Tabelle 5.10 unter der Annahme eines Ein-Faktor-Modells. Des Weiteren sind auch die entsprechenden Turnover der Strategien angegeben.

Tabelle 5.9: Turnover und um Transaktionskosten bereinigte MUGs der passiven und historischen Strategien gegenüber der impliziten Strategie auf Basis des Constant-Correlation-Ansatzes

\begin{tabular}{|c|c|c|c|c|c|c|}
\hline & \multicolumn{2}{|c|}{ MUG } & TO & \multicolumn{2}{|c|}{ MUG } & TO \\
\hline $\mathrm{N}=10$ & \multicolumn{3}{|c|}{$\mathrm{RRA}=2$} & \multicolumn{3}{|c|}{$\mathrm{RRA}=4$} \\
\hline $1 / \mathrm{N}$ & $3,07 \%$ & $(0,13)$ & $16,17 \%$ & $4,23 \%$ & $(0,08)$ & $16,17 \%$ \\
\hline Dow & $2,20 \%$ & $(0,21)$ & $14,80 \%$ & $3,02 \%$ & $(0,14)$ & $14,80 \%$ \\
\hline Dow_MCap & $3,76 \%$ & $(0,09)$ & $10,04 \%$ & $5,04 \%$ & $(0,05)$ & $10,04 \%$ \\
\hline Sample & $-0,54 \%$ & $(0,61)$ & $23,33 \%$ & $-0,02 \%$ & $(0,50)$ & $23,12 \%$ \\
\hline ConstCor & $0,51 \%$ & $(0,39)$ & $18,35 \%$ & $1,17 \%$ & $(0,27)$ & $18,33 \%$ \\
\hline Shrink_CC & $-0,25 \%$ & $(0,55)$ & $20,69 \%$ & $0,28 \%$ & $(0,44)$ & $20,60 \%$ \\
\hline Impl_ēC & & & $61,71 \%$ & & & $62,89 \%$ \\
\hline $\mathrm{N}=10$ & \multicolumn{3}{|c|}{$\mathrm{RRA}=6$} & \multicolumn{3}{|c|}{$\mathrm{RRA}=8$} \\
\hline $1 / \mathrm{N}$ & $5,35 \%$ & $(0,05)$ & $16,17 \%$ & $5,86 \%$ & $(0,06)$ & $16,17 \%$ \\
\hline Dow & $3,79 \%$ & $(0,11)$ & $14,80 \%$ & $3,92 \%$ & $(0,13)$ & $14,80 \%$ \\
\hline Dow_MCap & $6,28 \%$ & $(0,02)$ & $10,04 \%$ & $6,91 \%$ & $(0,03)$ & $10,04 \%$ \\
\hline Sample & $0,55 \%$ & $(0,38)$ & $23,00 \%$ & $0,62 \%$ & $(0,38)$ & $22,93 \%$ \\
\hline ConstCor & $1,86 \%$ & $(0,16)$ & $18,31 \%$ & $2,07 \%$ & $(0,17)$ & $18,30 \%$ \\
\hline Shrink_CC & $0,85 \%$ & $(0,32)$ & $20,54 \%$ & $0,95 \%$ & $(0,32)$ & $20,39 \%$ \\
\hline Impl_ēC & & & $64,16 \%$ & & & $65,88 \%$ \\
\hline $\mathrm{N}=20$ & \multicolumn{3}{|c|}{$\mathrm{RRA}=2$} & \multicolumn{3}{|c|}{$\mathrm{RRA}=4$} \\
\hline $1 / \mathrm{N}$ & $2,09 \%$ & $(0,27)$ & $9,57 \%$ & $3,31 \%$ & $(0,20)$ & $9,57 \%$ \\
\hline Dow & $1,66 \%$ & $(0,30)$ & $7,31 \%$ & $2,31 \%$ & $(0,25)$ & $7,31 \%$ \\
\hline Dow_MCap & $3,69 \%$ & $(0,14)$ & $4,88 \%$ & $4,73 \%$ & $(0,10)$ & $4,88 \%$ \\
\hline Sample & $0,24 \%$ & $(0,45)$ & $20,22 \%$ & $0,31 \%$ & $(0,45)$ & $20,23 \%$ \\
\hline ConstCor & $1,55 \%$ & $(0,23)$ & $15,32 \%$ & $1,97 \%$ & $(0,18)$ & $15,35 \%$ \\
\hline Shrink_CC & $0,53 \%$ & $(0,39)$ & $16,64 \%$ & $0,71 \%$ & $(0,38)$ & $16,68 \%$ \\
\hline Impl_CC & & & $66,67 \%$ & & & $68,13 \%$ \\
\hline $\mathrm{N}=20$ & \multicolumn{3}{|c|}{$\mathrm{RRA}=6$} & \multicolumn{3}{|c|}{$\mathrm{RRA}=8$} \\
\hline $1 / \mathrm{N}$ & $4,77 \%$ & $(0,12)$ & $9,57 \%$ & $6,32 \%$ & $(0,08)$ & $9,57 \%$ \\
\hline Dow & $3,12 \%$ & $(0,19)$ & $7,31 \%$ & $3,93 \%$ & $(0,16)$ & $7,31 \%$ \\
\hline Dow_MCap & $5,94 \%$ & $(0,06)$ & $4,88 \%$ & $7,16 \%$ & $(0,04)$ & $4,88 \%$ \\
\hline Sample & $0,55 \%$ & $(0,41)$ & $20,25 \%$ & $0,84 \%$ & $(0,37)$ & $20,29 \%$ \\
\hline ConstCor & $2,57 \%$ & $(0,13)$ & $15,38 \%$ & $3,21 \%$ & $(0,09)$ & $15,40 \%$ \\
\hline Shrink_CC & $1,10 \%$ & $(0,31)$ & $16,58 \%$ & $1,52 \%$ & $(0,25)$ & $16,54 \%$ \\
\hline Impl_ēC & & & $69,35 \%$ & & & $70,26 \%$ \\
\hline
\end{tabular}

Fortsetzung der Tabelle auf der nächsten Seite... 


\begin{tabular}{|c|c|c|c|c|c|c|}
\hline \multicolumn{7}{|c|}{. Tabelle 5.9 - Fortsetzung } \\
\hline & \multicolumn{2}{|c|}{ MUG } & TO & \multicolumn{2}{|c|}{ MUG } & TO \\
\hline $\mathrm{N}=30$ & \multicolumn{3}{|c|}{$\mathrm{RRA}=2$} & \multicolumn{3}{|c|}{$\mathrm{RRA}=4$} \\
\hline $1 / \mathrm{N}$ & $0,61 \%$ & $(0,43)$ & $5,84 \%$ & $2,58 \%$ & $(0,27)$ & $5,84 \%$ \\
\hline Dow & $0,18 \%$ & $(0,48)$ & $2,79 \%$ & $1,36 \%$ & $(0,35)$ & $2,79 \%$ \\
\hline Dow_MCap & $2,41 \%$ & $(0,24)$ & $3,37 \%$ & $3,60 \%$ & $(0,16)$ & $3,37 \%$ \\
\hline Sample & $0,78 \%$ & $(0,38)$ & $19,91 \%$ & $0,92 \%$ & $(0,35)$ & $19,88 \%$ \\
\hline ConstCor & $1,18 \%$ & $(0,29)$ & $14,39 \%$ & $1,76 \%$ & $(0,20)$ & $14,46 \%$ \\
\hline Shrink_CC & $0,73 \%$ & $(0,36)$ & $15,63 \%$ & $1,08 \%$ & $(0,30)$ & $15,68 \%$ \\
\hline Impl_ēC & & & $71,99 \%$ & & & $73,48 \%$ \\
\hline $\mathrm{N}=30$ & \multicolumn{3}{|c|}{$\mathrm{RRA}=6$} & \multicolumn{3}{|c|}{$\mathrm{RRA}=8$} \\
\hline $1 / \mathrm{N}$ & $4,80 \%$ & $(0,14)$ & $5,84 \%$ & $6,56 \%$ & $(0,09)$ & $5,84 \%$ \\
\hline Dow & $2,70 \%$ & $(0,24)$ & $2,79 \%$ & $3,50 \%$ & $(0,21)$ & $2,79 \%$ \\
\hline Dow_MCap & $4,89 \%$ & $(0,09)$ & $3,37 \%$ & $5,54 \%$ & $(0,08)$ & $3,37 \%$ \\
\hline Sample & $1,14 \%$ & $(0,33)$ & $19,89 \%$ & $0,66 \%$ & $(0,40)$ & $19,81 \%$ \\
\hline ConstCor & $2,39 \%$ & $(0,13)$ & $14,48 \%$ & $2,48 \%$ & $(0,14)$ & $14,59 \%$ \\
\hline Shrink_CC & $1,48 \%$ & $(0,24)$ & $15,67 \%$ & $1,27 \%$ & $(0,29)$ & $15,70 \%$ \\
\hline Impl_ $\bar{C} C$ & & & $74,77 \%$ & & & $76,23 \%$ \\
\hline
\end{tabular}

Diese Tabelle zeigt das Turnover sowie die annualisierten, um Transaktionskosten bereinigten MUGs der passiven und historischen Strategien gegenüber der impliziten Strategie auf Basis des ConstantCorrelation-Ansatzes für Investoren mit CRRA Nutzenfunktionen. In Klammern sind aus einem Bootstrap-Verfahren mit 10.000 Simulationen abgeleitete p-Werte auf die Hypothese angegeben, dass der entsprechende MUG größer null ist. Der out-of-sample Zeitraum beträgt Februar 1998 bis November 2010.

Die Ergebnisse aus dem vorherigen Abschnitt können auch bei Berücksichtigung von Transaktionskosten bestätigt werden. Lediglich im Falle des Anlageuniversums $N=10$ werden zusätzlich für die Risikoaversion 4 für die Sample Strategie und für die Risikoaversion 2 für den Shrinkage Ansatz negative MUGs erzielt. Im Vergleich zu der Tabelle 5.6 und Tabelle 5.8 sinkt die Vorteilhaftigkeit der impliziten Strategien jedoch in allen Fällen. Dies ist auch an den Signifikanzniveaus zu erkennen.

Eine Betrachtung des Turnovers zur Beurteilung der Transaktionskosten ist angemessen. Im Einklang mit den Ergebnissen aus der strategischen Asset Allokation aus Kapitel 3, Kempf, Korn und Saßning (2012) und DeMiguel, Plyakha, Uppal und Vilkov (2012) zeigt sich, dass die impliziten Strategien gegenüber den entsprechenden Benchmarks einen deutlich höheren Wert aufweisen und somit höhere MUGs ohne Betrachtung von Transaktionskosten besitzen. Der höhere Turnover impliziter Strategien lässt sich darauf zurückführen, dass diese im Gegensatz zu passiven oder historischen Strategien unmittelbar auf geänderte Marktbegebenheiten eingehen und die Asset Allokation dementsprechend direkt anpassen. Sie basieren ausschließlich auf den Informationen eines einzelnen Stichtages und "mitteln" somit keine historischen Renditezeitreihen. 
Des Weiteren ist $\mathrm{zu}$ bemerken, dass die passiven Strategien aufgrund ihrer Anlagestrategie am geringsten umgeschichtet werden. Für die historischen Benchmark Strategien hat die Strukturierung einen positiven Effekt auf den Turnover, wodurch der vollständig strukturierte Schätzer (ConstCor bzw. SingleFactor) den geringsten Turnover aller aktiven Strategien aufweist.

Tabelle 5.10: Turnover und um Transaktionskosten bereinigte MUGs der passiven und historischen Strategien gegenüber der impliziten Strategie auf Basis der Annahme eines Ein-FaktorModells

\begin{tabular}{|c|c|c|c|c|c|c|}
\hline & \multicolumn{2}{|c|}{ MUG } & TO & \multicolumn{2}{|c|}{ MUG } & TO \\
\hline $\mathrm{N}=10$ & \multicolumn{3}{|c|}{$\mathrm{RRA}=2$} & \multicolumn{3}{|c|}{$\mathrm{RRA}=4$} \\
\hline $1 / \mathrm{N}$ & $3,13 \%$ & $(0,13)$ & $16,17 \%$ & $4,19 \%$ & $(0,08)$ & $16,17 \%$ \\
\hline Dow & $2,25 \%$ & $(0,20)$ & $14,80 \%$ & $2,98 \%$ & $(0,15)$ & $14,80 \%$ \\
\hline Dow_MCap & $3,82 \%$ & $(0,10)$ & $10,04 \%$ & $5,00 \%$ & $(0,05)$ & $10,04 \%$ \\
\hline Sample & $-0,48 \%$ & $(0,59)$ & $23,33 \%$ & $-0,06 \%$ & $(0,52)$ & $23,12 \%$ \\
\hline SingleFactor & $1,17 \%$ & $(0,27)$ & $19,75 \%$ & $1,59 \%$ & $(0,20)$ & $19,75 \%$ \\
\hline Shrink_SF & $0,14 \%$ & $(0,47)$ & $20,72 \%$ & $0,54 \%$ & $(0,39)$ & $20,70 \%$ \\
\hline Impl_s & & & $62,27 \%$ & & & $63,15 \%$ \\
\hline $\mathrm{N}=10$ & \multicolumn{3}{|c|}{$\mathrm{RRA}=6$} & \multicolumn{3}{|c|}{$\mathrm{RRA}=8$} \\
\hline $1 / \mathrm{N}$ & $5,13 \%$ & $(0,06)$ & $16,17 \%$ & $5,82 \%$ & $(0,06)$ & $16,17 \%$ \\
\hline Dow & $3,57 \%$ & $(0,12)$ & $14,80 \%$ & $3,88 \%$ & $(0,13)$ & $14,80 \%$ \\
\hline Dow_MCap & $6,06 \%$ & $(0,04)$ & $10,04 \%$ & $6,87 \%$ & $(0,03)$ & $10,04 \%$ \\
\hline Sample & $0,33 \%$ & $(0,44)$ & $23,00 \%$ & $0,58 \%$ & $(0,40)$ & $22,93 \%$ \\
\hline SingleFactor & $1,96 \%$ & $(0,16)$ & $19,76 \%$ & $2,22 \%$ & $(0,15)$ & $19,75 \%$ \\
\hline Shrink_SF & $0,89 \%$ & $(0,32)$ & $20,70 \%$ & $1,16 \%$ & $(0,30)$ & $20,76 \%$ \\
\hline Impl_s & & & $64,01 \%$ & & & $64,84 \%$ \\
\hline $\mathrm{N}=20$ & \multicolumn{3}{|c|}{$\mathrm{RRA}=2$} & \multicolumn{3}{|c|}{$\mathrm{RRA}=4$} \\
\hline $1 / \mathrm{N}$ & $2,03 \%$ & $(0,29)$ & $9,57 \%$ & $3,39 \%$ & $(0,18)$ & $9,57 \%$ \\
\hline Dow & $1,60 \%$ & $(0,31)$ & $7,31 \%$ & $2,39 \%$ & $(0,25)$ & $7,31 \%$ \\
\hline Dow_MCap & $3,63 \%$ & $(0,16)$ & $4,88 \%$ & $4,81 \%$ & $(0,10)$ & $4,88 \%$ \\
\hline Sample & $0,19 \%$ & $(0,47)$ & $20,22 \%$ & $0,39 \%$ & $(0,43)$ & $20,23 \%$ \\
\hline SingleFactor & $1,28 \%$ & $(0,28)$ & $15,22 \%$ & $1,60 \%$ & $(0,24)$ & $15,27 \%$ \\
\hline Shrink_SF & $0,66 \%$ & $(0,38)$ & $16,58 \%$ & $0,89 \%$ & $(0,35)$ & $16,65 \%$ \\
\hline Impl_s & & & $67,30 \%$ & & & $68,09 \%$ \\
\hline $\mathrm{N}=20$ & \multicolumn{3}{|c|}{$\mathrm{RRA}=6$} & \multicolumn{3}{|c|}{$\mathrm{RRA}=8$} \\
\hline $1 / \mathrm{N}$ & $4,84 \%$ & $(0,12)$ & $9,57 \%$ & $6,39 \%$ & $(0,08)$ & $9,57 \%$ \\
\hline Dow & $3,19 \%$ & $(0,19)$ & $7,31 \%$ & $4,00 \%$ & $(0,16)$ & $7,31 \%$ \\
\hline Dow_MCap & $6,01 \%$ & $(0,06)$ & $4,88 \%$ & $7,23 \%$ & $(0,04)$ & $4,88 \%$ \\
\hline Sample & $0,62 \%$ & $(0,41)$ & $20,25 \%$ & $0,92 \%$ & $(0,36)$ & $20,29 \%$ \\
\hline SingleFactor & $1,95 \%$ & $(0,19)$ & $15,33 \%$ & $2,34 \%$ & $(0,15)$ & $15,31 \%$ \\
\hline Shrink_SF & $1,15 \%$ & $(0,31)$ & $16,68 \%$ & $1,41 \%$ & $(0,28)$ & $16,75 \%$ \\
\hline Impl_sF & & & $68,63 \%$ & & & $69,21 \%$ \\
\hline
\end{tabular}

Fortsetzung der Tabelle auf der nächsten Seite... 


\begin{tabular}{|c|c|c|c|c|c|c|}
\hline \multicolumn{7}{|c|}{ Tabelle 5.10 - Fortsetzung } \\
\hline & \multicolumn{2}{|c|}{ MUG } & TO & \multicolumn{2}{|c|}{ MUG } & TO \\
\hline $\mathrm{N}=30$ & \multicolumn{3}{|c|}{$\mathrm{RRA}=2$} & \multicolumn{3}{|c|}{$\mathrm{RRA}=4$} \\
\hline $1 / \mathrm{N}$ & $0,55 \%$ & $(0,44)$ & $5,84 \%$ & $2,66 \%$ & $(0,25)$ & $5,84 \%$ \\
\hline Dow & $0,12 \%$ & $(0,48)$ & $2,79 \%$ & $1,43 \%$ & $(0,34)$ & $2,79 \%$ \\
\hline Dow_MCap & $2,35 \%$ & $(0,25)$ & $3,37 \%$ & $3,68 \%$ & $(0,16)$ & $3,37 \%$ \\
\hline Sample & $0,72 \%$ & $(0,38)$ & $19,91 \%$ & $1,00 \%$ & $(0,34)$ & $19,88 \%$ \\
\hline SingleFactor & $0,49 \%$ & $(0,40)$ & $15,43 \%$ & $0,87 \%$ & $(0,34)$ & $15,48 \%$ \\
\hline Shrink_SF & $0,43 \%$ & $(0,43)$ & $16,37 \%$ & $0,72 \%$ & $(0,37)$ & $16,40 \%$ \\
\hline Impl_SF & & & $72,44 \%$ & & & $73,20 \%$ \\
\hline $\mathrm{N}=30$ & \multicolumn{3}{|c|}{$\mathrm{RRA}=6$} & \multicolumn{3}{|c|}{$\mathrm{RRA}=8$} \\
\hline $1 / \mathrm{N}$ & $4,91 \%$ & $(0,14)$ & $5,84 \%$ & $7,05 \%$ & $(0,08)$ & $5,84 \%$ \\
\hline Dow & $2,81 \%$ & $(0,23)$ & $2,79 \%$ & $3,99 \%$ & $(0,18)$ & $2,79 \%$ \\
\hline Dow_MCap & $4,99 \%$ & $(0,09)$ & $3,37 \%$ & $6,02 \%$ & $(0,06)$ & $3,37 \%$ \\
\hline Sample & $1,24 \%$ & $(0,32)$ & $19,89 \%$ & $1,15 \%$ & $(0,33)$ & $19,81 \%$ \\
\hline SingleFactor & $1,21 \%$ & $(0,30)$ & $15,50 \%$ & $1,26 \%$ & $(0,30)$ & $15,56 \%$ \\
\hline Shrink_SF & $0,96 \%$ & $(0,34)$ & $16,53 \%$ & $0,87 \%$ & $(0,36)$ & $16,60 \%$ \\
\hline Impl_SF & & & $73,60 \%$ & & & $74,17 \%$ \\
\hline
\end{tabular}

Diese Tabelle zeigt das Turnover sowie die annualisierten, um Transaktionskosten bereinigten MUGs der passiven und historischen Strategien gegenüber der impliziten Strategie auf Basis der Annahme eines Ein-Faktor-Modells für Investoren mit CRRA Nutzenfunktionen. In Klammern sind aus einem BootstrapVerfahren mit 10.000 Simulationen abgeleitete p-Werte auf die Hypothese angegeben, dass der entsprechende MUG größer null ist. Der out-of-sample Zeitraum beträgt Februar 1998 bis November 2010.

\subsubsection{Unterteilung des out-of-sample Zeitraums nach der reali- sierten Volatilität des Marktes}

Aus den Ergebnissen der Tabelle 5.7 wurde deutlich, dass sich die impliziten Strategien durch eine signifikant geringere realisierte Volatilität gegenüber den betrachteten Benchmarks auszeichnen. Um dieses Ergebnis weiter zu untersuchen, wird der out-of-sample Zeitraum in zwei Subperioden unterteilt. Sortiert nach der realisierten Volatilität des preisgewichteten Index (Dow) - als Proxy für den Markt -, fasst der Zeitraum "Calm" diejenige Hälfte der Perioden mit den geringsten Werten zusammen und kann als "ruhige" Marktphase interpretiert werden. Der Zeitraum "Non-Calm" entspricht derjenigen Hälfte mit den höchsten realisierten Volatilitäten und entspricht somit dem Fall eines "unruhigen" Marktumfeldes.

Die "unruhige" Phase zeichnet sich durch starke Preisanpassungen und sich ändernde Erwartungen an die zukünftige Verteilung der Assets durch die Marktteilnehmer aus. Die implizite Portfoliostrategie kann die Asset Allokation auf die geänderten Marktbegebenheiten und Erwartungen aufgrund ihrer vorwärtsgerichteten Eigenschaft unmittelbar an- 
passen. ${ }^{32}$ Historische Vergleichsstrategien hingegen berücksichtigen diese Veränderungen lediglich verzögert und reagieren erst verspätet auf die sich ändernde Marktbeschaffenheit. Die implizite Strategie sollte daher im Vergleich zwischen "ruhigen" und "unruhigen" Marktphasen insbesondere in den "unruhigen" Phasen den übrigen Strategien vorzuziehen sein.

Zur weiteren Motivation dieser Behauptung liefert die folgende Heuristik auch ein technisches Argument. Im Rahmen eines Minimum-Varianz-Ansatzes zur Bestimmung der optimalen Asset Allokation werden Assets stärker übergewichtet, die geringe Volatilitäten und Kovarianzen aufweisen. Bei Verwendung des Constant-Correlation-Ansatzes gilt für die Kovarianz

$$
M_{2_{i j}}=\rho^{M_{2}} \cdot \sqrt{\mu_{i}^{(2)} \mu_{j}^{(2)}} \quad \text { für alle } i \neq j,
$$

woraus sich ableiten lässt, dass diejenigen Assets eine geringe Kovarianz im Verhältnis zu den übrigen Paaren haben, welche auch die geringsten Volatilitäten aufweisen. Im Rahmen der Annahme eines Ein-Faktor-Modells zeigen Clarke, De Silva und Thorley (2010), dass ein Minimum-Varianz-Ansatz einer "low-beta" Strategie gleicht, also einer Übergewichtung von Assets mit einem geringen $\beta$-Faktor. Der implizit identifizierte $\beta$ Faktor von Unternehmen $i$ basiert auf der impliziten Volatilität des Unternehmens, wobei sich eine geringe Volatilität in einen geringen $\beta$-Faktor übersetzt. ${ }^{33}$

Die verwendeten Strukturen zur Identifikation der impliziten Kovarianzmatrix haben somit zur Konsequenz, dass sich die optimale Asset Allokation stark an der impliziten Volatilität ausrichtet. Daraus lässt sich ableiten, dass die realisierte Portfoliovolatilität aus der "richtigen" Identifikation von Assets mit zukünftig geringer (realisierter) Volatilität resultiert. Empirisch ist dies in der "unruhigen" Marktphase ausgeprägter zu erwarten.

Die Resultate in Tabelle 5.11 zeigen, dass bedeutende Unterschiede für das $N=10$ Portfolio im Vergleich zwischen den Subperioden bestehen. So ist beispielhaft für die Risikoaversion 4 der MUG für den gleichgewichteten Index $(1 / \mathrm{N})$ in der ruhigen Phase 2,69\% $(2,55 \%$ für RRA $=8)$ und 6,76\% (9,68\% für RRA = 8) in der unruhigen. Der Unterschied lässt sich auch an den ermittelten p-Werten erkennen. So sind die MUGs ausschließlich in der unruhigen Periode signifikant. Auch gegenüber den historischen Benchmarks ist die impliziten Strategie in den unruhigen Phasen klar vorzuziehen. Für den risikoaverseren Investor $(\mathrm{RRA}=8)$ ist hingegen ein Nutzenverlust, insbesondere gegenüber dem Stichprobenschätzer, in der ruhigen Marktphase zu beobachten.

32 Vgl. Kempf, Korn und Saßning (2012).

33 Die $\beta$-Bestimmung auf Basis der Volatilität übersetzt sich in eine Kovarianzmatrix mit einer konstanten Korrelation (vgl. Saßning (2012) Kapitel 3.4.5). Die paarweise Korrelation entspricht dem Anteil $c_{t}$ an der systematischen Varianz der einzelnen Assets. 
Tabelle 5.11: MUGs der passiven und historischen Strategien gegenüber der impliziten Strategie auf Basis des Constant-Correlation-Ansatzes für zwei Subperioden

\begin{tabular}{|c|c|c|c|c|c|c|c|c|}
\hline & \multicolumn{2}{|c|}{$\mathrm{RRA}=4$} & \multicolumn{2}{|c|}{$\mathrm{RRA}=8$} & \multicolumn{2}{|c|}{$\mathrm{RRA}=4$} & \multicolumn{2}{|c|}{$\mathrm{RRA}=8$} \\
\hline $\mathrm{N}=10$ & \multicolumn{4}{|c|}{ Calm } & \multicolumn{4}{|c|}{ Non-Calm } \\
\hline $1 / \mathrm{N}$ & $2,69 \%$ & $(0,15)$ & $2,55 \%$ & $(0,21)$ & $6,76 \%$ & $(0,10)$ & $9,68 \%$ & $(0,05)$ \\
\hline Dow & $2,27 \%$ & $(0,18)$ & $1,87 \%$ & $(0,27)$ & $4,86 \%$ & $(0,16)$ & $6,75 \%$ & $(0,11)$ \\
\hline Dow_MCap & $1,77 \%$ & $(0,26)$ & $1,88 \%$ & $(0,30)$ & $9,34 \%$ & $(0,04)$ & $12,25 \%$ & $(0,02)$ \\
\hline Sample & $-0,21 \%$ & $(0,53)$ & $-1,48 \%$ & $(0,72)$ & $1,09 \%$ & $(0,36)$ & $3,32 \%$ & $(0,15)$ \\
\hline ConstCor & $0,13 \%$ & $(0,47)$ & $-0,68 \%$ & $(0,60)$ & $3,19 \%$ & $(0,13)$ & $5,40 \%$ & $(0,04)$ \\
\hline Shrink_CC & $0,17 \%$ & $(0,47)$ & $-0,87 \%$ & $(0,63)$ & $1,37 \%$ & $(0,31)$ & $3,48 \%$ & $(0,13)$ \\
\hline $\mathrm{N}=20$ & \multicolumn{4}{|c|}{ Calm } & \multicolumn{4}{|c|}{ Non-Calm } \\
\hline $1 / \mathrm{N}$ & $-2,19 \%$ & $(0,71)$ & $-1,33 \%$ & $(0,62)$ & $9,87 \%$ & $(0,06)$ & $13,96 \%$ & $(0,03)$ \\
\hline Dow & $-1,52 \%$ & $(0,64)$ & $-0,90 \%$ & $(0,57)$ & $7,37 \%$ & $(0,10)$ & $9,38 \%$ & $(0,07)$ \\
\hline Dow_MCap & $-1,09 \%$ & $(0,60)$ & $-0,33 \%$ & $(0,51)$ & $11,71 \%$ & $(0,03)$ & $14,80 \%$ & $(0,01)$ \\
\hline Sample & $-1,08 \%$ & $(0,63)$ & $-1,20 \%$ & $(0,64)$ & $2,76 \%$ & $(0,24)$ & $3,71 \%$ & $(0,18)$ \\
\hline ConstCor & $0,86 \%$ & $(0,37)$ & $1,13 \%$ & $(0,34)$ & $4,27 \%$ & $(0,11)$ & $6,24 \%$ & $(0,04)$ \\
\hline Shrink_CC & $-0,40 \%$ & $(0,54)$ & $-0,30 \%$ & $(0,54)$ & $3,00 \%$ & $(0,19)$ & $4,29 \%$ & $(0,10)$ \\
\hline $\mathrm{N}=30$ & \multicolumn{4}{|c|}{ Calm } & \multicolumn{4}{|c|}{ Non-Calm } \\
\hline $1 / \mathrm{N}$ & $-4,92 \%$ & $(0,92)$ & $-4,00 \%$ & $(0,85)$ & $11,26 \%$ & $(0,05)$ & $16,75 \%$ & $(0,02)$ \\
\hline Dow & $-4,48 \%$ & $(0,90)$ & $-3,73 \%$ & $(0,84)$ & $8,55 \%$ & $(0,07)$ & $11,18 \%$ & $(0,05)$ \\
\hline Dow_MCap & $-1,72 \%$ & $(0,67)$ & $-1,17 \%$ & $(0,60)$ & $10,31 \%$ & $(0,04)$ & $12,79 \%$ & $(0,02)$ \\
\hline Sample & $0,03 \%$ & $(0,49)$ & $0,08 \%$ & $(0,48)$ & $3,05 \%$ & $(0,23)$ & $2,49 \%$ & $(0,30)$ \\
\hline ConstCor & $1,74 \%$ & $(0,24)$ & $2,11 \%$ & $(0,22)$ & $3,18 \%$ & $(0,17)$ & $4,26 \%$ & $(0,12)$ \\
\hline Shrink_CC & $1,35 \%$ & $(0,29)$ & $1,47 \%$ & $(0,28)$ & $2,20 \%$ & $(0,25)$ & $2,53 \%$ & $(0,24)$ \\
\hline
\end{tabular}

Diese Tabelle zeigt die annualisierten MUGs der passiven und historischen Strategien gegenüber der impliziten Strategie auf Basis des Constant-Correlation-Ansatzes für Investoren mit CRRA Nutzenfunktionen mit Risikoaversionen 4 und 8. In Klammern sind aus einem Bootstrap-Verfahren mit 10.000 Simulationen abgeleitete p-Werte auf die Hypothese angegeben, dass der entsprechende MUG größer null ist. Der out-of-sample Zeitraum von Februar 1998 bis November 2010 ist unterteilt in zwei Subperioden. Die Unterteilung erfolgt nach der realisierten Volatilität des preisgewichteten Index (Dow). Die Subperiode "Calm" umfasst diejenige Hälfte der Perioden mit den geringsten realisierten Volatilitäten, die Subperiode "Non-Calm" die übrige Hälfte des out-of-sample Zeitraums.

Für die Portfolios $N=20$ und $N=30$ kann die Beobachtung noch klarer bestätigt werden. In den unruhigen Marktphasen ist die implizite Strategie allen Benchmarks deutlich vorzuziehen und weist insbesondere im Vergleich zu den passiven Strategien eine signifikant bessere Performance auf. Dies gilt auch in der Calm-Periode für die aktiven Vergleichsstrategien auf Basis historischer Daten für das Anlageuniserum $N=30$. Jedoch zeigt sich ein differenziertes Bild für die passiven Benchmarks und im Speziellen für den gleichgewichteten Index $(1 / \mathrm{N})$. In der ruhigen Phase hat dieser einen negativen MUG und ist gegenüber der impliziten Strategie als lohnenswerter einzustufen. Die Gründe für die bessere Performance zeigen die Excess Returns und realisierten Volatilitäten in Tabelle 5.12 . 
Tabelle 5.12: Excess Returns und realisierte Volatilitäten der passiven, historischen und impliziten Strategien auf Basis des Constant-Correlation-Ansatzes für zwei Subperioden

\begin{tabular}{|c|c|c|c|c|c|c|c|c|}
\hline & \multicolumn{4}{|c|}{$\hat{\mu}$} & \multicolumn{2}{|c|}{$\hat{\mu}$} & \multicolumn{2}{|c|}{$\hat{\sigma}$} \\
\hline & \multicolumn{4}{|c|}{ Calm } & \multicolumn{4}{|c|}{ Non-Calm } \\
\hline $\mathrm{N}=10$ & \multicolumn{4}{|c|}{$\mathrm{RRA}=4$} & \multicolumn{4}{|c|}{$\mathrm{RRA}=4$} \\
\hline $1 / \mathrm{N}$ & $11,03 \%$ & $(0,46)$ & $11,67 \%$ & $(0,00)$ & $-14,94 \%$ & $(0,39)$ & $27,28 \%$ & $(0,00)$ \\
\hline Dow & $11,29 \%$ & $(0,52)$ & $11,13 \%$ & $(0,04)$ & $-13,78 \%$ & $(0,52)$ & $26,17 \%$ & $(0,00)$ \\
\hline Dow_MCap & $12,13 \%$ & $(0,76)$ & $11,93 \%$ & $(0,00)$ & $-17,47 \%$ & $(0,19)$ & $27,98 \%$ & $(0,00)$ \\
\hline Sample & $13,29 \%$ & $(0,90)$ & $11,45 \%$ & $(0,00)$ & $-10,41 \%$ & $(0,94)$ & $24,19 \%$ & $(0,00)$ \\
\hline ConstCor & $13,09 \%$ & $(0,97)$ & $11,47 \%$ & $(0,00)$ & $-12,31 \%$ & $(0,58)$ & $23,43 \%$ & $(0,00)$ \\
\hline Shrink_CC & $12,97 \%$ & $(0,99)$ & $11,36 \%$ & $(0,00)$ & $-10,69 \%$ & $(0,99)$ & $23,50 \%$ & $(0,00)$ \\
\hline Impl_CC & $13,00 \%$ & & $10,73 \%$ & & $-10,64 \%$ & & $21,69 \%$ & \\
\hline $\mathrm{N}=10$ & \multicolumn{4}{|c|}{$\mathrm{RRA}=8$} & \multicolumn{4}{|c|}{$\mathrm{RRA}=8$} \\
\hline $1 / \mathrm{N}$ & $11,03 \%$ & $(0,80)$ & $11,67 \%$ & $(0,05)$ & $-14,94 \%$ & $(0,36)$ & $27,28 \%$ & $(0,00)$ \\
\hline Dow & $11,29 \%$ & $(0,86)$ & $11,13 \%$ & $(0,51)$ & $-13,78 \%$ & $(0,48)$ & $26,17 \%$ & $(0,00)$ \\
\hline Dow_MCap & $12,13 \%$ & $(0,95)$ & $11,93 \%$ & $(0,01)$ & $-17,47 \%$ & $(0,18)$ & $27,98 \%$ & $(0,00)$ \\
\hline Sample & $13,51 \%$ & $(0,54)$ & $11,46 \%$ & $(0,12)$ & $-10,63 \%$ & $(0,91)$ & $24,22 \%$ & $(0,00)$ \\
\hline ConstCor & $13,07 \%$ & $(0,66)$ & $11,48 \%$ & $(0,09)$ & $-12,30 \%$ & $(0,51)$ & $23,45 \%$ & $(0,00)$ \\
\hline Shrink_CC & $13,07 \%$ & $(0,65)$ & $11,37 \%$ & $(0,17)$ & $-10,69 \%$ & $(0,89)$ & $23,52 \%$ & $(0,00)$ \\
\hline Impl_ēC & $11,89 \%$ & & $11,25 \%$ & & $-10,25 \%$ & & $21,85 \%$ & \\
\hline $\mathrm{N}=20$ & \multicolumn{4}{|c|}{$\mathrm{RRA}=4$} & \multicolumn{4}{|c|}{$\mathrm{RRA}=4$} \\
\hline $1 / \mathrm{N}$ & $14,60 \%$ & $(0,50)$ & $11,50 \%$ & $(0,00)$ & $-13,81 \%$ & $(0,27)$ & $27,85 \%$ & $(0,00)$ \\
\hline Dow & $13,75 \%$ & $(0,63)$ & $10,86 \%$ & $(0,14)$ & $-12,66 \%$ & $(0,31)$ & $25,91 \%$ & $(0,00)$ \\
\hline Dow_MCap & $13,43 \%$ & $(0,70)$ & $11,56 \%$ & $(0,00)$ & $-16,10 \%$ & $(0,14)$ & $27,75 \%$ & $(0,00)$ \\
\hline Sample & $12,86 \%$ & $(0,74)$ & $10,93 \%$ & $(0,10)$ & $-9,11 \%$ & $(0,61)$ & $23,07 \%$ & $(0,00)$ \\
\hline ConstCor & $11,03 \%$ & $(0,75)$ & $11,15 \%$ & $(0,01)$ & $-9,66 \%$ & $(0,47)$ & $22,75 \%$ & $(0,00)$ \\
\hline Shrink_CC & $12,17 \%$ & $(0,91)$ & $11,01 \%$ & $(0,05)$ & $-8,93 \%$ & $(0,61)$ & $22,54 \%$ & $(0,00)$ \\
\hline Impl_CC & $11,88 \%$ & & $10,77 \%$ & & $-7,12 \%$ & & $21,10 \%$ & \\
\hline $\mathrm{N}=20$ & \multicolumn{4}{|c|}{$\mathrm{RRA}=8$} & \multicolumn{4}{|c|}{$\mathrm{RRA}=8$} \\
\hline $1 / \mathrm{N}$ & $14,60 \%$ & $(0,55)$ & $11,50 \%$ & $(0,01)$ & $-13,81 \%$ & $(0,27)$ & $27,85 \%$ & $(0,00)$ \\
\hline Dow & $13,75 \%$ & $(0,69)$ & $10,86 \%$ & $(0,20)$ & $-12,66 \%$ & $(0,31)$ & $25,91 \%$ & $(0,00)$ \\
\hline Dow_MCap & $13,43 \%$ & $(0,76)$ & $11,56 \%$ & $(0,01)$ & $-16,10 \%$ & $(0,14)$ & $27,75 \%$ & $(0,00)$ \\
\hline Sample & $13,05 \%$ & $(0,77)$ & $10,96 \%$ & $(0,14)$ & $-9,29 \%$ & $(0,57)$ & $23,10 \%$ & $(0,00)$ \\
\hline ConstCor & $10,96 \%$ & $(0,65)$ & $11,17 \%$ & $(0,02)$ & $-9,65 \%$ & $(0,46)$ & $22,78 \%$ & $(0,00)$ \\
\hline Shrink_CC & $12,11 \%$ & $(0,97)$ & $11,03 \%$ & $(0,07)$ & $-8,89 \%$ & $(0,60)$ & $22,58 \%$ & $(0,01)$ \\
\hline Impl_ēC & $12,20 \%$ & & $10,84 \%$ & & $-6,98 \%$ & & $21,47 \%$ & \\
\hline $\mathrm{N}=30$ & \multicolumn{4}{|c|}{$\mathrm{RRA}=4$} & \multicolumn{4}{|c|}{$\mathrm{RRA}=4$} \\
\hline $1 / \mathrm{N}$ & $15,56 \%$ & $(0,15)$ & $11,59 \%$ & $(0,00)$ & $-12,94 \%$ & $(0,37)$ & $29,32 \%$ & $(0,00)$ \\
\hline Dow & $14,97 \%$ & $(0,18)$ & $11,17 \%$ & $(0,01)$ & $-12,30 \%$ & $(0,33)$ & $26,51 \%$ & $(0,00)$ \\
\hline Dow_MCap & $12,08 \%$ & $(0,58)$ & $11,59 \%$ & $(0,00)$ & $-13,77 \%$ & $(0,26)$ & $27,78 \%$ & $(0,00)$ \\
\hline Sample & $10,01 \%$ & $(1,00)$ & $10,65 \%$ & $(0,20)$ & $-8,88 \%$ & $(0,66)$ & $22,67 \%$ & $(0,00)$ \\
\hline ConstCor & $8,37 \%$ & $(0,51)$ & $11,06 \%$ & $(0,01)$ & $-7,89 \%$ & $(0,82)$ & $22,51 \%$ & $(0,00)$ \\
\hline
\end{tabular}

Fortsetzung der Tabelle auf der nächsten Seite... 


\begin{tabular}{|c|c|c|c|c|c|c|c|c|}
\hline \multicolumn{9}{|c|}{... Tabelle 5.12 - Fortsetzung } \\
\hline & \multicolumn{2}{|c|}{$\hat{\mu}$} & \multicolumn{2}{|c|}{$\hat{\sigma}$} & \multicolumn{2}{|c|}{$\hat{\mu}$} & \multicolumn{2}{|c|}{$\hat{\sigma}$} \\
\hline & \multicolumn{4}{|c|}{ Calm } & \multicolumn{4}{|c|}{ Non-Calm } \\
\hline Shrink_CC & $8,60 \%$ & $(0,56)$ & $10,87 \%$ & $(0,04)$ & $-7,47 \%$ & $(0,91)$ & $22,15 \%$ & $(0,00)$ \\
\hline Impl_ēC & $10,01 \%$ & & $10,64 \%$ & & $-7,05 \%$ & & $20,71 \%$ & \\
\hline $\mathrm{N}=30$ & \multicolumn{4}{|c|}{$\mathrm{RRA}=8$} & \multicolumn{4}{|c|}{$\mathrm{RRA}=8$} \\
\hline $1 / \mathrm{N}$ & $15,56 \%$ & $(0,17)$ & $11,59 \%$ & $(0,00)$ & $-12,94 \%$ & $(0,47)$ & $29,32 \%$ & $(0,00)$ \\
\hline Dow & $14,97 \%$ & $(0,21)$ & $11,17 \%$ & $(0,02)$ & $-12,30 \%$ & $(0,45)$ & $26,51 \%$ & $(0,00)$ \\
\hline Dow_MCap & $12,08 \%$ & $(0,62)$ & $11,59 \%$ & $(0,00)$ & $-13,77 \%$ & $(0,35)$ & $27,78 \%$ & $(0,00)$ \\
\hline Sample & $10,12 \%$ & $(0,99)$ & $10,68 \%$ & $(0,25)$ & $-8,95 \%$ & $(0,87)$ & $22,67 \%$ & $(0,00)$ \\
\hline ConstCor & $8,24 \%$ & $(0,45)$ & $11,10 \%$ & $(0,01)$ & $-7,79 \%$ & $(0,91)$ & $22,53 \%$ & $(0,00)$ \\
\hline Shrink_CC & $8,49 \%$ & $(0,50)$ & $10,91 \%$ & $(0,05)$ & $-7,38 \%$ & $(0,83)$ & $22,19 \%$ & $(0,03)$ \\
\hline Impl_ēC & $10,16 \%$ & & $10,71 \%$ & & $-8,19 \%$ & & $21,24 \%$ & \\
\hline
\end{tabular}

Diese Tabelle zeigt die Excess Returns und realisierten Volatilitäten der passiven, historischen und impliziten Strategien auf Basis des Constant-Correlation-Ansatzes für Investoren mit CRRA Nutzenfunktionen mit Risikoaversionen 4 und 8. Alle Werte sind annualisiert. In Klammern sind p-Werte von t-Tests auf die Hypothese angegeben, dass die implizite Strategie denselben Excess Return bzw. dieselbe realisierte Volatilität besitzt. Der out-of-sample Zeitraum von Februar 1998 bis November 2010 ist unterteilt in zwei Subperioden. Die Unterteilung erfolgt nach der realisierten Volatilität des preisgewichteten Index (Dow). Die Subperiode "Calm" umfasst diejenige Hälfte der Perioden mit den geringsten realisierten Volatilitäten, die Subperiode "Non-Calm" die übrige Hälfte des out-of-sample Zeitraums.

Für das Anlageuniversum $N=10$ zeigen die Ergebnisse in der Calm-Periode für die realisierte Volatilität unter den verschiedenen Strategien nur geringe absolute Abweichungen. Zwar liefert die implizite Strategie den geringsten bzw. zweitgeringsten Wert, dieser ist aber nur 1,2 Prozentpunkte geringer als der höchste Wert $(\mathrm{RRA}=4)$, in diesem Fall der nach der Marktkapitalisierung gewichtete Index. Die Ergebnisse sind daher vor allem durch den Excess Return getrieben. In den unruhigen Marktphasen zeigt sich ein verändertes Bild. Der Unterschied in der realisierten Volatilität zwischen dem höchsten und dem geringsten Wert, erneut der marktkapitalisierte Index und der implizite Ansatz, beträgt absolut über 6 Prozentpunkte. Im Unterschied zu den aktiven historischen Strategien, welche eine deutliche Verbesserung gegenüber den passiven Strategien darstellen, beträgt der absolut erwartete Abstand mindestens 1,6 Prozentpunkte. Die Excess Returns sind für die aktiven Strategien - Sample, ConstCor und Implizit - vergleichbar und sind deutlich höher als diejenigen der entsprechenden passiven Benchmarks.

Im Vergleich zwischen ruhiger und unruhiger Marktphase wird ein negatives Risk-Return Verhältnis festgestellt. In Phasen niedriger Volatilität werden durch alle Strategien positive Excess Returns erzielt, in den unruhigen Phasen hingegen negative. Diese Beobachtung steht im Gegensatz zu theoretischen Überlegungen, dass eine hohe Volatilität eine höhere Kompensation für die Übernahme dieses Risikos bedeutet. Empirisch konnte jedoch ein 
negatives Verhältnis nachgewiesen werden, wodurch die gute Performance von MinimumVarianz-Strategien in der Asset Allokation erklärt werden kann. ${ }^{34}$

Für die erweiterten Portfolios $N=20$ und $N=30$ lassen sich folgende Beobachtungen festhalten. In den ruhigen Phasen erzielt der gleichgewichtete Index $(1 / \mathrm{N})$ den höchsten Excess Return über alle Strategien, innerhalb der aktiven der Stichprobenschätzer oder der implizite Ansatz. Die realisierte Volatilität der impliziten Strategie ist auch in den ruhigen Marktphasen die signifikant geringste, jedoch absolut gemessen nur marginal, mit der Ausnahme des Stichprobenschätzers, welcher ein vergleichbaren Wert erzielt. In den unruhigen Marktphasen hingegen überzeugt die implizite Strategie unter beiden Metriken, durch einen geringen negativen Excess Return sowie durch die geringste realisierte Volatilität. Die Beobachtung des negativen Risk-Return Verhältnisses wird in den erweiterten Anlageuniversen bestätigt.

Zusammenfassend zeigt die Unterteilung in Phasen hoher und niedriger Volatilität, dass die impliziten Strategien insbesondere in den unruhigen Phasen den Benchmark Strategien vorzuziehen sind. In den ruhigen Phasen sind diese auch im Vergleich zu den Benchmarks auf Basis historischer Daten als lohnenswert oder vergleichbar einzustufen, jedoch liefert unter diesen Marktbeschaffenheiten eine passive 1/N Strategie die überzeugendste Performance.

Für die Annahme eines Ein-Faktor-Modells zeigt Tabelle 5.14 die entsprechenden MUGs, welche die bisherigen Ergebnisse und Beobachtungen bestätigen.

34 Vgl. u.a. Glosten, Jagannathen und Runkle (1993), Ang, Hodrick, Xing und Zhang (2006), Ang, Hodrick, Xing und Zhang (2009) und Clarke, De Silva und Thorley (2010). 
Tabelle 5.14: MUGs der passiven und historischen Strategien gegenüber der impliziten Strategie auf Basis der Annahme eines Ein-Faktor-Modells für zwei Subperioden

\begin{tabular}{lcccccccc}
\hline & \multicolumn{3}{c}{ RRA=4 } & \multicolumn{2}{c}{ RRA=8 } & \multicolumn{2}{c}{ RRA=4 } & \multicolumn{2}{c}{ RRA=8 } \\
\hline 1/N & $2,59 \%$ & $(0,18)$ & $2,61 \%$ & $(0,20)$ & $6,79 \%$ & $(0,10)$ & $9,55 \%$ & $(0,05)$ \\
Dow & $2,16 \%$ & $(0,22)$ & $1,93 \%$ & $(0,26)$ & $4,89 \%$ & $(0,17)$ & $6,62 \%$ & $(0,12)$ \\
Dow_MCap & $1,66 \%$ & $(0,28)$ & $1,94 \%$ & $(0,28)$ & $9,37 \%$ & $(0,04)$ & $12,12 \%$ & $(0,02)$ \\
Sample & $-0,32 \%$ & $(0,56)$ & $-1,43 \%$ & $(0,71)$ & $1,12 \%$ & $(0,36)$ & $3,18 \%$ & $(0,16)$ \\
SingleFactor & $1,56 \%$ & $(0,23)$ & $0,69 \%$ & $(0,38)$ & $2,64 \%$ & $(0,19)$ & $4,49 \%$ & $(0,08)$ \\
Shrink_SF & $0,83 \%$ & $(0,36)$ & $-0,18 \%$ & $(0,52)$ & $1,25 \%$ & $(0,35)$ & $3,26 \%$ & $(0,15)$ \\
\hline \multicolumn{1}{c}{ N=20 } & \multicolumn{9}{c}{ Calm } & & & Non-Calm & \\
\hline 1/N & $-2,01 \%$ & $(0,69)$ & $-1,21 \%$ & $(0,60)$ & $9,85 \%$ & $(0,06)$ & $13,97 \%$ & $(0,03)$ \\
Dow & $-1,34 \%$ & $(0,62)$ & $-0,78 \%$ & $(0,56)$ & $7,35 \%$ & $(0,09)$ & $9,38 \%$ & $(0,07)$ \\
Dow_MCap & $-0,92 \%$ & $(0,57)$ & $-0,21 \%$ & $(0,51)$ & $11,70 \%$ & $(0,03)$ & $14,81 \%$ & $(0,01)$ \\
Sample & $-0,90 \%$ & $(0,61)$ & $-1,08 \%$ & $(0,63)$ & $2,75 \%$ & $(0,24)$ & $3,72 \%$ & $(0,18)$ \\
SingleFactor & $1,21 \%$ & $(0,32)$ & $1,22 \%$ & $(0,32)$ & $3,23 \%$ & $(0,19)$ & $4,54 \%$ & $(0,11)$ \\
Shrink_SF & $0,26 \%$ & $(0,44)$ & $0,13 \%$ & $(0,47)$ & $2,70 \%$ & $(0,23)$ & $3,69 \%$ & $(0,16)$ \\
\hline \multicolumn{1}{c}{ N=30 } & \multicolumn{9}{c}{ Calm } & & & Non-Calm & \\
\hline 1/N & $-4,88 \%$ & $(0,91)$ & $-3,95 \%$ & $(0,85)$ & $11,36 \%$ & $(0,05)$ & $17,56 \%$ & $(0,01)$ \\
Dow & $-4,43 \%$ & $(0,89)$ & $-3,69 \%$ & $(0,84)$ & $8,66 \%$ & $(0,06)$ & $11,99 \%$ & $(0,04)$ \\
Dow_MCap & $-1,68 \%$ & $(0,66)$ & $-1,13 \%$ & $(0,60)$ & $10,41 \%$ & $(0,04)$ & $13,59 \%$ & $(0,02)$ \\
Sample & $0,08 \%$ & $(0,48)$ & $0,12 \%$ & $(0,47)$ & $3,16 \%$ & $(0,22)$ & $3,30 \%$ & $(0,23)$ \\
SingleFactor & $1,80 \%$ & $(0,24)$ & $1,90 \%$ & $(0,24)$ & $1,36 \%$ & $(0,35)$ & $2,09 \%$ & $(0,29)$ \\
Shrink_SF & $1,15 \%$ & $(0,33)$ & $1,15 \%$ & $(0,33)$ & $1,65 \%$ & $(0,33)$ & $1,98 \%$ & $(0,31)$ \\
\hline
\end{tabular}

Diese Tabelle zeigt die annualisierten MUGs der passiven und historischen Strategien gegenüber der impliziten Strategie auf Basis der Annahme eines Ein-Faktor-Modells für Investoren mit CRRA Nutzenfunktionen mit Risikoaversionen 4 und 8. In Klammern sind aus einem Bootstrap-Verfahren mit 10.000 Simulationen abgeleitete p-Werte auf die Hypothese angegeben, dass der entsprechende MUG größer null ist. Der out-of-sample Zeitraum von Februar 1998 bis November 2010 ist unterteilt in zwei Subperioden. Die Unterteilung erfolgt nach der realisierten Volatilität des preisgewichteten Index (Dow). Die Subperiode "Calm" umfasst diejenige Hälfte der Perioden mit den geringsten realisierten Volatilitäten, die Subperiode "Non-Calm" die übrige Hälfte des out-of-sample Zeitraums.

\subsubsection{Nutzen von höheren impliziten Momenten}

Zur Beantwortung der weiteren zentralen Forschungsfrage dieser Arbeit, welchen Beitrag implizite Schiefe und Kurtosis bei der Asset Allokation mit höheren Momenten outof-sample leisten, wird folgend der Strategie Impl_CC das implizite Minimum-VarianzPortfolio gegenüber gestellt. Dieses wird durch die Lösung des Asset Allokationsproblems (5.1) gegeben, indem zusätzlich die Matrizen $M_{3}$ und $M_{4}$ gleich null gesetzt werden. Die Matrix $M_{2}$ wird entsprechend der impliziten Strategie Impl_CC mit $M_{2_{C C}}^{i m p l}$ identifiziert. Das resultierende Minimum-Varianz-Portfolio ist unabhängig von dem spezifischen Risikoaversionsparameter $\gamma$ und somit gleich für alle Investoren. 
Tabelle 5.15 zeigt die MUGs aus dem Vergleich der Strategien, wobei ein positiver Wert einen Nutzenvorteil der impliziten Strategie auf Basis der höheren Momente (Impl_CC) gegenüber dem impliziten Minimum-Varianz-Portfolio darstellt. Die Ergebnisse werden auch für die aus dem vorherigen Abschnitt definierten Subperioden "Calm" und "NonCalm" angegeben. "Full" bezeichnet in diesem Fall den gesamten out-of-sample Zeitraum.

Tabelle 5.15: MUGs für die implizite Strategie Impl_CC gegenüber dem Minimum-Varianz-Portfolio auf Basis des Constant-Correlation-Ansatzes für den gesamten Zeitraum und zwei Subperioden

\begin{tabular}{lcccccccc}
\hline \multicolumn{3}{c}{$\mathrm{RRA}=2$} & \multicolumn{2}{c}{$\mathrm{RRA}=4$} & \multicolumn{2}{c}{$\mathrm{RRA}=6$} & \multicolumn{2}{c}{$\mathrm{RRA}=8$} \\
\hline \multicolumn{1}{c}{$\mathrm{N}=10$} & & & & & & & & \\
\hline Full & $0,10 \%$ & $(0,25)$ & $0,16 \%$ & $(0,27)$ & $0,22 \%$ & $(0,28)$ & $-0,23 \%$ & $(0,57)$ \\
Calm & $0,07 \%$ & $(0,30)$ & $0,13 \%$ & $(0,28)$ & $0,20 \%$ & $(0,26)$ & $-1,03 \%$ & $(0,65)$ \\
Non-Calm & $0,13 \%$ & $(0,32)$ & $0,18 \%$ & $(0,35)$ & $0,24 \%$ & $(0,34)$ & $0,44 \%$ & $(0,27)$ \\
\hline \multicolumn{1}{c}{$\mathrm{N}=20$} & & & & & & & & \\
\hline Full & $-0,06 \%$ & $(0,54)$ & $-0,29 \%$ & $(0,64)$ & $-0,34 \%$ & $(0,66)$ & $-0,34 \%$ & $(0,65)$ \\
Calm & $-0,41 \%$ & $(0,71)$ & $-0,97 \%$ & $(0,73)$ & $-0,97 \%$ & $(0,71)$ & $-1,01 \%$ & $(0,71)$ \\
Non-Calm & $0,29 \%$ & $(0,15)$ & $0,35 \%$ & $(0,19)$ & $0,23 \%$ & $(0,32)$ & $0,24 \%$ & $(0,36)$ \\
\hline \multicolumn{1}{c}{$\mathrm{N}=30$} & & & & & & & & \\
\hline Full & $-0,23 \%$ & $(0,78)$ & $-0,49 \%$ & $(0,78)$ & $-0,66 \%$ & $(0,84)$ & $-1,40 \%$ & $(0,89)$ \\
Calm & $-0,44 \%$ & $(0,72)$ & $-0,98 \%$ & $(0,71)$ & $-1,03 \%$ & $(0,72)$ & $-1,10 \%$ & $(0,73)$ \\
Non-Calm & $-0,01 \%$ & $(0,54)$ & $-0,02 \%$ & $(0,52)$ & $-0,31 \%$ & $(0,67)$ & $-1,67 \%$ & $(0,77)$ \\
\hline
\end{tabular}

Diese Tabelle zeigt die annualisierten MUGs für die implizite Strategie Impl_CC gegenüber dem Minimum-Varianz-Portfolio auf Basis des Constant-Correlation-Ansatzes für Investoren mit CRRA Nutzenfunktionen. In Klammern sind aus einem Bootstrap-Verfahren mit 10.000 Simulationen abgeleitete p-Werte auf die Hypothese angegeben, dass der entsprechende MUG größer null ist. Der out-of-sample Zeitraum beträgt Februar 1998 bis November 2010 und wird mit "Full" bezeichnet. Des Weiteren sind zwei Subperioden angegeben. Die Unterteilung erfolgt nach der realisierten Volatilität des preisgewichteten Index (Dow). Die Subperiode "Calm" umfasst diejenige Hälfte der Perioden mit den geringsten realisierten Volatilitäten, die Subperiode "Non-Calm" die übrige Hälfte des out-of-sample Zeitraums.

Für das Anlageuniversum $N=10$ wird für den gesamten Zeitraum ("Full") ein positiver MUG erzielt und zeigt die Vorteilhaftigkeit aus der Verwendung der höheren Momente durch die bessere Approximation des Nutzens auch out-of-sample. Das Ergebnis zeigt sich auch für die Subperioden robust. Lediglich für die Risikoaversion 8 wird ein negativer MUG für den gesamten Zeitraum sowie für die Calm Periode bestimmt. Ein Vergleich zwischen den Subperioden deutet nur einen geringen Unterschied der Performance der Impl_CC Strategie an.

Für die erweiterten Universen $N=20$ und $N=30$ wird hingegen ein umgekehrtes Resultat ermittelt. Die Vorteilhaftigkeit der Impl_CC ist nicht mehr gegeben und das von der Risikoaversion unabhängige Minimum-Varianz-Portfolio erzielt über den gesamten Zeit- 
raum ein höheres Nutzenniveau. Der Vergleich zwischen den Subperioden zeigt jedoch wesentliche Unterschiede der Performance. In den unruhigen Phasen ist die Performance der Impl_CC Strategie im Vergleich zu der ruhigen Periode deutlich verbessert. Insbesondere für das $N=20$ Universum zeigen die Resultate in der unruhigen Phase positive MUGs, was einem out-of-sample Nutzenvorteil aus der Verwendung höherer Momente in der Asset Allokation gleichzusetzen ist.

Mögliche Gründe für die beobachteten Unterschiede zwischen den Strategien lassen sich aus den durchschnittlichen Differenzen in Excess Returns und realisierter Volatilität aus Tabelle 5.16 ableiten.

Tabelle 5.16: Differenzen der Excess Returns und der realisierten Volatilität zwischen der impliziten Strategie Impl_CC und dem Minimum-Varianz-Portfolio auf Basis des ConstantCorrelation-Ansatzes für den gesamten Zeitraum und zwei Subperioden

\begin{tabular}{|c|c|c|c|c|c|c|c|c|}
\hline & \multicolumn{2}{|c|}{$\Delta \hat{\mu}$} & \multicolumn{2}{|c|}{$\Delta \hat{\sigma}$} & \multicolumn{2}{|c|}{$\Delta \hat{\mu}$} & \multicolumn{2}{|c|}{$\Delta \hat{\sigma}$} \\
\hline $\mathrm{N}=10$ & \multicolumn{4}{|c|}{$\mathrm{RRA}=4$} & \multicolumn{4}{|c|}{$\mathrm{RRA}=8$} \\
\hline Full & $0,17 \%$ & $(0,54)$ & $0,16 \%$ & $(0,00)$ & $-0,19 \%$ & $(0,83)$ & $0,43 \%$ & $(0,01)$ \\
\hline Calm & $0,13 \%$ & $(0,65)$ & $0,16 \%$ & $(0,01)$ & $-0,98 \%$ & $(0,56)$ & $0,68 \%$ & $(0,10)$ \\
\hline Non-Calm & $0,20 \%$ & $(0,66)$ & $0,17 \%$ & $(0,00)$ & $0,60 \%$ & $(0,45)$ & $0,33 \%$ & $(0,00)$ \\
\hline $\mathrm{N}=20$ & \multicolumn{4}{|c|}{$\mathrm{RRA}=4$} & \multicolumn{4}{|c|}{$\mathrm{RRA}=8$} \\
\hline Full & $-0,19 \%$ & $(0,73)$ & $0,29 \%$ & $(0,00)$ & $0,04 \%$ & $(0,95)$ & $0,54 \%$ & $(0,00)$ \\
\hline Calm & $-0,79 \%$ & $(0,43)$ & $0,45 \%$ & $(0,15)$ & $-0,48 \%$ & $(0,65)$ & $0,53 \%$ & $(0,05)$ \\
\hline Non-Calm & $0,42 \%$ & $(0,29)$ & $0,22 \%$ & $(0,00)$ & $0,57 \%$ & $(0,38)$ & $0,58 \%$ & $(0,00)$ \\
\hline $\mathrm{N}=30$ & \multicolumn{4}{|c|}{$\mathrm{RRA}=4$} & \multicolumn{4}{|c|}{$\mathrm{RRA}=8$} \\
\hline Full & $-0,43 \%$ & $(0,42)$ & $0,30 \%$ & $(0,01)$ & $-0,93 \%$ & $(0,30)$ & $0,65 \%$ & $(0,00)$ \\
\hline Calm & $-0,83 \%$ & $(0,41)$ & $0,46 \%$ & $(0,15)$ & $-0,69 \%$ & $(0,51)$ & $0,53 \%$ & $(0,06)$ \\
\hline Non-Calm & $-0,03 \%$ & $(0,94)$ & $0,24 \%$ & $(0,00)$ & $-1,17 \%$ & $(0,42)$ & $0,77 \%$ & $(0,02)$ \\
\hline
\end{tabular}

Diese Tabelle zeigt die Differenzen für den Excess Return und der realisierten Volatilität zwischen der impliziten Strategie Impl_CC und dem Minimum-Varianz-Portfolio auf Basis des Constant-CorrelationAnsatzes für Investoren mit CRRA Nutzenfunktionen mit Risikoaversionen 4 und 8. In Klammern sind p-Werte von t-Tests auf die Hypothese angegeben, dass die Strategien denselben Excess Return bzw. dieselbe realisierte Volatilität besitzen. Der out-of-sample Zeitraum beträgt Februar 1998 bis November 2010 und wird mit "Full" bezeichnet. Des Weiteren sind zwei Subperioden angegeben. Die Unterteilung erfolgt nach der realisierten Volatilität des preisgewichteten Index (Dow). Die Subperiode "Calm" umfasst diejenige Hälfte der Perioden mit den geringsten realisierten Volatilitäten, die Subperiode "Non-Calm" die übrige Hälfte des out-of-sample Zeitraums.

Für den gesamten Zeitraum ("Full") sowie für die Subperioden lassen sich insbesondere anhand der Differenz in der realisierten Volatilität Unterschiede erkennen. So liefert im Durchschnitt das Minimum-Varianz-Portfolio, im Einklang mit dessen Definition, eine geringere realisierte Volatilität. In den überwiegenden Fällen ist die Differenz sogar statistisch signifikant. 
Die Unterschiede in den Excess Returns sind teilweise positiv bzw. negativ. Jedoch lässt sich beobachten, dass diese insbesondere in der ruhigen Marktphase geringer sind als in der unruhigen Periode. Die p-Werte deuten jedoch an, dass die Unterschiede nicht stark voneinander variieren. Eine Analyse der Gewichte der Strategien, worauf die unterschiedliche Performance in den Subperioden zurückzuführen ist, liefert Tabelle 5.17.

Tabelle 5.17: Durchschnittlicher absoluter Abstand zwischen dem gleichgewichteten Index $(1 / \mathrm{N})$ und den Gewichten der impliziten Strategie Impl_CC und des Minimum-Varianz-Portfolios auf Basis des Constant-Correlation-Ansatzes für den gesamten Zeitraum und zwei Subperioden

\begin{tabular}{lccccc}
\hline & MVP & RRA=2 & RRA=4 & RRA=6 & RRA=8 \\
\hline \multicolumn{1}{c}{$\mathrm{N}=10$} & & & & & \\
\hline Full & $9,84 \%$ & $10,09 \%$ & $10,28 \%$ & $10,48 \%$ & $10,66 \%$ \\
Calm & $8,48 \%$ & $8,74 \%$ & $8,93 \%$ & $9,14 \%$ & $9,35 \%$ \\
Non-Calm & $11,21 \%$ & $11,45 \%$ & $11,65 \%$ & $11,83 \%$ & $11,99 \%$ \\
\hline$\quad$ N=20 & & & & \\
\hline Full & $6,24 \%$ & $6,38 \%$ & $6,49 \%$ & $6,59 \%$ & $6,68 \%$ \\
Calm & $5,67 \%$ & $5,81 \%$ & $5,93 \%$ & $6,03 \%$ & $6,13 \%$ \\
Non-Calm & $6,82 \%$ & $6,95 \%$ & $7,05 \%$ & $7,15 \%$ & $7,24 \%$ \\
\hline \multicolumn{1}{c}{$\mathrm{N}=30$} & & & & & \\
\hline Full & $4,65 \%$ & $4,73 \%$ & $4,79 \%$ & $4,86 \%$ & $4,92 \%$ \\
Calm & $4,34 \%$ & $4,42 \%$ & $4,49 \%$ & $4,56 \%$ & $4,62 \%$ \\
Non-Calm & $4,97 \%$ & $5,04 \%$ & $5,10 \%$ & $5,16 \%$ & $5,22 \%$ \\
\hline
\end{tabular}

Diese Tabelle zeigt den durchschnittlichen absoluten Abstand zwischen dem gleichgewichteten Index $(1 / N)$ und den Gewichten der impliziten Strategien Impl_CC und des Minimum-Varianz-Portfolios "MVP" auf Basis des Constant-Correlation-Ansatzes für Investoren mit CRRA Nutzenfunktionen. Der out-of-sample Zeitraum beträgt Februar 1998 bis November 2010 und wird mit "Full" bezeichnet. Des Weiteren sind zwei Subperioden angegeben. Die Unterteilung erfolgt nach der realisierten Volatilität des preisgewichteten Index (Dow). Die Subperiode "Calm" umfasst diejenige Hälfte der Perioden mit den geringsten realisierten Volatilitäten, die Subperiode "Non-Calm" die übrige Hälfte des out-of-sample Zeitraums.

Die Tabelle zeigt die durchschnittlichen, im Querschnitt gemittelten absoluten Abstände zwischen den Gewichten der Impl_CC Strategie und dem gleichgewichteten Portfolio $(1 / \mathrm{N})$ sowie die entsprechenden Abstände für das Minimum-Varianz-Portfolio (MVP) gegenüber dem 1/N Benchmark. Aus den Werten wird unmittelbar ersichtlich, dass im Durchschnitt für jedes Anlageuniversum und jede Periode die Gewichte des MinimumVarianz-Portfolios "gleichgewichteter" sind als die Gewichte der Impl_CC Strategie. Dass durch beide aktive Strategien in unruhigen Zeiten ein weniger gleichgewichteteres Portfolio gewählt wird, kann auf eine höhere Variation im Querschnitt der impliziten Volatilitäten zurückgeführt werden, welche sich unmittelbar in extremeren Gewichten in der Asset Allokation ausdrücken. 
Mit den Ergebnissen aus dem vorherigen Abschnitt aus Tabelle 5.11 lassen sich die Resultate aus dem Unterschied zwischen den impliziten Strategien erklären. Im Einklang mit den Beobachtungen für die 1/N Strategie, liefert die gleichgewichtetere implizite Strategie insbesondere in der ruhigen Phase eine verbesserte Performance.

Eine Ursache, weshalb die Impl_CC Strategie ein Portfolio mit "extremeren" Gewichten wählt, lässt sich unter anderem darauf zurückführen, dass eine hohe implizite Volatilität auch eine hohe implizite Kurtosis impliziert, was wiederum dazu führt, dass diese Assets aus Nutzensicht unattraktiver eingeschätzt werden. Dieses ist insbesondere daran zu erkennen, dass die Gewichte umso "extremer" werden, je risikoaverser der Investor ist und die spezifischen Assets somit noch unattraktiver wirken.

Abschließend zeigt Tabelle 5.18 die entsprechenden MUGs für die Annahme eines EinFaktor-Modells. Das Minimum-Varianz-Portfolio wird in diesem Fall auf Basis der Matrix $M_{2_{S F}}^{i m p l}$ gebildet. Bisherige Ergebnisse und Beobachtungen aus dem Constant-CorrelationAnsatz werden bestätigt.

Tabelle 5.18: MUGs für die implizite Strategie Impl_SF gegenüber dem Minimum-Varianz-Portfolio auf Basis der Annahme eines Ein-Faktor-Modells für den gesamten Zeitraum und zwei Subperioden

\begin{tabular}{lcccccccc}
\hline \multicolumn{1}{c}{$\mathrm{RRA}=2$} & \multicolumn{2}{c}{$\mathrm{RRA}=4$} & \multicolumn{2}{c}{$\mathrm{RRA}=6$} & \multicolumn{2}{c}{$\mathrm{RRA}=8$} \\
\hline \multicolumn{1}{c}{$\mathrm{N}=10$} & & & & & & & \\
\hline Full & $0,01 \%$ & $(0,47)$ & $-0,04 \%$ & $(0,53)$ & $-0,15 \%$ & $(0,59)$ & $-0,43 \%$ & $(0,67)$ \\
Calm & $-0,07 \%$ & $(0,59)$ & $-0,19 \%$ & $(0,61)$ & $-0,50 \%$ & $(0,65)$ & $-1,19 \%$ & $(0,66)$ \\
Non-Calm & $0,09 \%$ & $(0,34)$ & $0,10 \%$ & $(0,38)$ & $0,16 \%$ & $(0,33)$ & $0,21 \%$ & $(0,32)$ \\
\hline \multicolumn{1}{c}{$\mathrm{N}=20$} & & & & & & & & \\
\hline Full & $-0,25 \%$ & $(0,64)$ & $-0,32 \%$ & $(0,67)$ & $-0,36 \%$ & $(0,68)$ & $-0,33 \%$ & $(0,66)$ \\
Calm & $-0,74 \%$ & $(0,69)$ & $-0,88 \%$ & $(0,67)$ & $-0,94 \%$ & $(0,67)$ & $-0,97 \%$ & $(0,67)$ \\
Non-Calm & $0,25 \%$ & $(0,11)$ & $0,21 \%$ & $(0,23)$ & $0,16 \%$ & $(0,34)$ & $0,21 \%$ & $(0,31)$ \\
\hline \multicolumn{1}{c}{$\mathrm{N}=30$} & & & & & & & & \\
\hline Full & $-0,37 \%$ & $(0,75)$ & $-0,51 \%$ & $(0,80)$ & $-0,65 \%$ & $(0,87)$ & $-1,03 \%$ & $(0,90)$ \\
Calm & $-0,76 \%$ & $(0,69)$ & $-0,95 \%$ & $(0,71)$ & $-0,99 \%$ & $(0,70)$ & $-1,07 \%$ & $(0,70)$ \\
Non-Calm & $0,02 \%$ & $(0,48)$ & $-0,08 \%$ & $(0,63)$ & $-0,34 \%$ & $(0,77)$ & $-1,00 \%$ & $(0,80)$ \\
\hline
\end{tabular}

Diese Tabelle zeigt die annualisierten MUGs für die implizite Strategie Impl_SF gegenüber dem Minimum-Varianz-Portfolio auf Basis der Annahme eines Ein-Faktor-Modells für Investoren mit CRRA Nutzenfunktionen. In Klammern sind aus einem Bootstrap-Verfahren mit 10.000 Simulationen abgeleitete p-Werte auf die Hypothese angegeben, dass der entsprechende MUG größer null ist. Der out-of-sample Zeitraum beträgt Februar 1998 bis November 2010 und wird mit "Full" bezeichnet. Des Weiteren sind zwei Subperioden angegeben. Die Unterteilung erfolgt nach der realisierten Volatilität des preisgewichteten Index (Dow). Die Subperiode "Calm" umfasst diejenige Hälfte der Perioden mit den geringsten realisierten Volatilitäten, die Subperiode "Non-Calm" die übrige Hälfte des out-of-sample Zeitraums. 


\subsection{Diskussion}

In diesem Kapitel wurde zur Untersuchung der zentralen Forschungsfragen dieser Arbeit die taktische Asset Allokation mit impliziten höheren Momenten betrachtet. Hierzu wurde zunächst aufgezeigt, wie mit Hilfe strukturierender Annahmen an die Verteilung der riskanten Anlagewerte im Querschnitt eine Identifikation der Kovarianz-, Koschiefe- und Kokurtosismatrix aus einem vollständig impliziten Ansatz geleistet werden kann. In diesem Zusammenhang wurden einerseits die Annahme konstanter Korrelationen, andererseits die Annahme einer Ein-Faktor Struktur herangezogen. Insbesondere die Identifikation der Koschiefe- und Kokurtosismatrix über die Annahme konstanter Korrelationen aus einem vollständig impliziten Ansatz stellt einen neu entwickelten Beitrag zur bestehenden Literatur dar. Die empirische Durchführung der taktischen Asset Allokation erfolgte auf Basis eines Datensatzes für die Mitglieder des DJIA.

Für die aus dem Modell abgeleiteten Korrelationen für die Matrizen höherer Ordnung wurde anhand des Datensatzes die zeitliche Struktur untersucht und mit entsprechenden realisierten Werten verglichen. Für eine Beurteilung der Prognosequalität wurden auch historische Schätzer ausgewertet. Zusammenfassend wurden folgende Beobachtungen gemacht: Für die konstante Korrelation der Kovarianzmatrix wurde eine Überschätzung durch den impliziten Wert im Einklang mit der bestehenden Literatur ${ }^{35}$ beobachtet. Die Prognose realisierter Werte wird ausschließlich durch den impliziten Ansatz zufriedenstellend geleistet. Die zeitliche Struktur der Schiefe-Korrelation kann weder durch den impliziten noch durch den historischen Schätzer wiedergegeben werden, der historische zeigt jedoch eine deutlich geringere Verzerrung. Die implizite Kurtosis-Korrelation wird wiederum nur durch den impliziten Wert prognostiziert, der historische Schätzer weist aber eine geringere Verzerrung auf. Ein Vergleich der impliziten Korrelationen untereinander deutet eine hohe Abhängigkeit dieser an. In diesem Zusammenhang wurde eine antizyklische zeitliche Struktur zwischen den impliziten Korrelationen auf Basis der Varianz und der Schiefe festgestellt. Zwischen den Korrelationen auf Basis der Varianz und der Kurtosis wurde hingegen kein zeitvariabler Anteil beobachtet.

Für die anschließende Portfoliostudie zur Untersuchung der taktischen Asset Allokation wurde das Design aus Martellini und Ziemann (2010) übernommen und ein erweiterter Minimum-Varianz-Ansatz getestet. Die Beurteilung der Performance der impliziten Strategien erfolgte einerseits im Vergleich zu passiven Benchmarks sowie zu Strategien auf Basis historischer Renditezeitreihen. Die Ergebnisse zeigen, dass die impliziten Strategien den Benchmark Strategien vorzuziehen sind. Die Resultate erweisen sich als robust

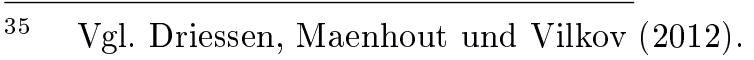


für unterschiedliche Risikoaversionen und Anlageuniversen sowie bei Hinzunahme von Transaktionskosten. Es wurde dabei aufgezeigt, dass die Ergebnisse insbesondere auf eine signifikant geringere realisierte Volatilität zurückzuführen sind.

Eine Unterteilung des out-of-sample Zeitraumes in eine "ruhige" und "unruhige" Marktphase anhand der realisierten Volatilität des Marktes zeigte, dass die implizite Strategie insbesondere in der unruhigen Phase den übrigen Strategien vorzuziehen ist. In den ruhigen Phasen lieferte jedoch das gleichgewichtete Portfolio $(1 / \mathrm{N})$ eine verbesserte Performance und ist unter diesen Marktbegebenheiten den impliziten Portfolio Strategien vorzuziehen. Zusammenfassend lassen die Ergebnisse dieser Studie die zentrale Forschungsfrage, ob implizite Informationen im Rahmen der taktischen Asset Allokation mit höheren Momenten vorteilhaft sind, bejahen.

Zur Untersuchung, welchen Beitrag die Berücksichtigung der impliziten Schiefe und Kurtosis zur Performance der impliziten Strategien leistet, wurde diesen die Performance des implizit bestimmten Minimum-Varianz-Portfolios gegenübergestellt. Martellini und Ziemann (2010) stellen in diesem Zusammenhang auf Basis historischer Renditezeitreihen eine Verschlechterung bzw. einen Nutzenverlust fest. Für den impliziten Ansatz ist dieses Ergebnis im Wesentlichen zu bestätigen, jedoch wurde bei der Unterteilung des Zeitraumes in die oben definierten Subperioden beobachtet, dass in den "unruhigen" Marktphasen die impliziten Strategien bei Berücksichtigung der impliziten Schiefe und Kurtosis eine verbesserte Performance erzielen. Als Grund hierfür wurde eine "extremere" optimale Vermögensverteilung identifiziert, welche unter anderem auf eine zusätzliche Abneigung gegenüber Kurtosisrisiken zurückzuführen ist. In den ruhigen Phasen hingegen überzeugt die Performance des Minimum-Varianz-Portfolios.

Insgesamt lässt sich im Einklang mit den Arbeiten von DeMiguel, Plyakha, Uppal und Vilkov (2012) und Kempf, Korn und Saßning (2012), welche die taktische Asset Allokation mit impliziten Informationen für Investoren mit $\mu-\sigma$ Nutzenfunktionen untersuchen, festhalten, dass auch im Rahmen der Asset Allokation mit höheren Momenten implizite Informationen enorme Potentiale aufweisen. Diese Erkenntnis hat auch für die Praxis bedeutende Relevanz, da sie Implikationen für die Komposition von Indices und Anlagefonds hat.

Die empirische Untersuchung der zentralen Forschungsfragen ist im Rahmen dieser Arbeit abgeschlossen. Das folgende Kapitel fasst die wesentlichen Erkenntnisse zusammen und gibt einen Ausblick auf weitere, sich anschließende Forschungsfragen. 


\section{Kapitel 6}

\section{Schlussbetrachtung und Ausblick}

Die vorliegende Arbeit hat die Asset Allokation mit höheren Momenten und impliziten Informationen untersucht. Die Beurteilung der zentralen Forschungsfragen, ob implizite Informationen out-of-sample einen Nutzenvorteil gegenüber historischen und passiven Benchmark Strategien haben sowie welchen Einfluss die implizite Schiefe und Kurtosis auf die out-of-sample Performance haben, erfolgte im Rahmen der strategischen und taktischen Asset Allokation.

Als zentrale Ergebnisse der empirischen Studien können festgehalten werden, dass im Vergleich zu historischen Benchmark Strategien implizite Informationen in der Asset Allokation enorme Potentiale bergen. Insbesondere wurde festgestellt, dass die impliziten Ansätze bezüglich der realisierten Volatilität überzeugen. Im Vergleich zu passiven Anlageformen und im Speziellen zur 1/N Strategie, konnte lediglich im Rahmen der taktischen Asset Allokation ein Nutzenvorteil erzielt werden. Jedoch lieferte die 1/N Strategie in Zeiten eines ruhigen Marktumfeldes eine verbesserte Performance gegenüber dem impliziten Ansatz.

Diese Erkenntnis steht im Einklang mit der Untersuchung von aktiven Portfoliostrategien auf Basis historischer Renditezeitreihen und ermutigt für weitere Forschungsfragen. So sollte in Analogie zu Tu und Zhou (2011) eine Portfolio Strategie basierend auf einer Mischung aus implizitem und passivem Ansatz formuliert werden. Diese Mischung könnte sich z. B. an makroökonomischen Daten orientieren, um eine Übergewichtung des passiven Anteils in ruhigen Marktphasen bzw. eine stärkere Gewichtung des impliziten Ansatzes in unruhigen Zeiten zu erzielen. Diese "Timing" Strategien könnten bei entsprechender Formulierung weiterhin auf einem vollständig impliziten Ansatz fußen.

Zur Beurteilung, welchen Einfluss implizite Schiefe und Kurtosis auf die Asset Allokation haben, wurden im Rahmen der strategischen Asset Allokation zwei gegensätzliche Beobachtungen getätigt. Bei Betrachtung eines Anlageuniversums, welches aus risikofreier 
Anlage, Aktienindex und einer langfristigen Anleihe besteht, konnte kein Einfluss bei der Gewichtung des riskanten Anteils des optimalen Portfolios beobachtet werden, bei Hinzunahme einer Rohstoffklasse im Asset-Mix hingegen varriiert die Komposition signifikant. Dieses Ergebnis hat im Rahmen der strategischen Asset Allokation weitreichende Konsequenzen, da es zeigt, dass sich die Risikoaffinität eines Investors nicht ausschließlich in der Aufteilung des Vermögens in einen riskanten und risikofreien Anteil ausdrückt, sondern auch in der Komposition des riskanten Portfolioanteils. Für praktische Anwendungen, z. B. für private und staatliche Anlagefonds, hat dies zur unmittelbaren Folge, dass das Tangentialportfolio nicht für alle Investoren als optimale riskante Anlage einzustufen ist.

Im Rahmen der taktischen Asset Allokation wurde der Einfluss der höheren Momente im Vergleich zum Minimum-Varianz-Portfolio untersucht. Insgesamt wurde im Wesentlichen eine Verschlechterung der Performance bei Berücksichtigung von impliziter Schiefe und Kurtosis festgestellt. Die Wirkung drückte sich in extremeren Portfoliogewichten im Vergleich zum Minimum-Varianz-Portfolio aus, was für die Performance zur Konsequenz hatte, dass in unruhigen bzw. volatilen Marktphasen teilweise ein Nutzenvorteil gegenüber dem Minimum-Varianz-Portfolio erzielt werden konnte, in ruhigen Phasen jedoch ein Nutzennachteil.

Zusammenfassend lässt sich insbesondere die Vorteilhaftigkeit der Verwendung von impliziten Informationen in der Asset Allokation gegenüber historischen Benchmark Strategien festhalten. Der Beurteilung des Nutzens für die out-of-sample Performance aus den höheren impliziten Momenten Schiefe und Kurtosis ist nicht eindeutig und bedarf weiterer Untersuchungen. Weiterführend schließen sich an die empirischen Studien aus Kapitel 3 bis 5 unmittelbar verschiedene Forschungsfragen an.

In Kapitel 3, welches die strategische Asset Allokation mit impliziten Verteilungen thematisierte, wurde die Annahme getroffen, dass die Investoren keine Portfolioumschichtungen während des Anlagehorizonts vornehmen können. Diese Annahme stellt im Rahmen der strategischen Asset Allokation eine Einschränkung dar und sollte in weiteren Studien aufgegeben werden. ${ }^{1}$ Als historische Benchmark Strategien werden in diesem Zusammenhang vor allem VAR(1)-Prozesse mit unterschiedlichen a-priori Verteilungen betrachtet. ${ }^{2}$

Eine weitere Untersuchung sollte an die präsentierte Methodik zur Konstruktion mehrdimensionaler impliziter Dichten für die Prognose realisierter Verteilungen anknüpfen.

$1 \quad$ Für eine Einführung in die Asset Allokation für Investoren mit mehrperiodigen Anlagehorizonten siehe unter anderem Campbell und Viceira (2001), Campbell, Chan und Viceira (2003) oder DeJong, Schotman und Werker (2008).

2 Vgl. hierzu z. B. Campbell und Viceira (2001), Campbell, Chan und Viceira (2003), Jurek und Viceira (2011), Hoevenaars, Molenaar, Schotman und Steenkamp (2007), Diris, Palm und Schotman (2009), Fugazza, Guidolin und Nicodano (2010) oder Doron und Avramov (2002). 
Hierzu müssten insbesondere unterschiedliche Copulas und verschiedene Horizonte zur Schätzung der Risikoaversion des repräsentativen Investors betrachtet werden. Das Vorgehen sollte sich an Shackleton, Taylor und Yu (2010) und Anagnou-Basioudis, Bedendo, Hodges und Tompkins (2005) orientieren.

Kapitel 4 hat das Konzept der risikoadjustierten modellfreien impliziten Momente eingeführt. Die präsentierte Methodik kann in einer Vielzahl von weiteren Anwendungen genutzt werden. Beispielsweise sollte die abgeleitete Markt-Risikoprämie wie in Duan und Zhang (2010) und Karoui (2011) zur Prognose realisierter Werte weiter untersucht werden. Auch könnte die Methodik dazu dienen, die Risikoaversion des repräsentativen Investors über eine exakte Herleitung des Volatilitäts-Spreads aus Bakshi und Kapadia (2003) oder mit GMM-Verfahren zu bestimmen. Eine Betrachtung von weiteren Nutzenfunktionen zur Beschreibung des repräsentativen Investors, z. B. über Epstein-Zin Präferenzen wie in Eraker (2008) oder Bollerslev, Tauchen und Zhou (2009), könnten zu neuen Erkenntnissen über die Wirkung der Risikoadjustierung führen. Der theoretische Beitrag, die Präsentation einer analytischen Lösung der Risikoadjustierung impliziter Momente, sollte wiederum dazu genutzt werden, die implizite Volatilität nach Britten-Jones und Neuberger (2000) und Jiang und Tian (2005) zu transformieren, indem die erwartete quadratische Variation unter dem physischen Maß betrachtet wird.

Neben den schon erwähnten Anwendungen für Timing Strategien sind die aus Kapitel 5 zur Untersuchung der taktischen Asset Allokation mit impliziten höheren Momenten hergeleiteten impliziten Korrelationen von besonderem Interesse für weitere Forschungsfragen. Die identifizierten Abhängigkeitsstrukturen in den höheren Momenten können beispielsweise zur Formulierung von Copulas dienen. Des Weiteren ermöglichen sie eine vollständige Dekomposition der impliziten Schiefe und Kurtosis des entsprechenden Indexes. Die Dekompositionen bzw. die individuellen Beiträge der Mitglieder zu den IndexMomenten können schließlich in der Asset Allokation für Sorting Portfolios wie in Conrad, Dittmar und Ghysel (2013) genutzt werden. 



\section{Literaturverzeichnis}

Adler, T., Kritzman, M. (2007): "Meanvariance versus full-scale optimisation: In and out of sample". Journal of Asset Management, 7(5), 302-311.

Agarwal, V., Bakshi, G., Huij, J. (2009): "Do higher-moment equity risks explain hedge fund returns?" Robert H. Smith School Research Paper No. RHS 06-153.

Albuquerque, R. (2012): "Skewness in Stock Returns: Reconciling the Evidence on Firm Versus Aggregate Returns". Review of Financial Studies, Seiten 1630-1673.

Alsmeyer, G. (2003): Wahrscheinlichkeitstheorie 3. Auflage (Skripten zur Mathematischen Statistik Nr. 30, Münster).

Amaya, D., Christoffersen, P., Jacobs, K., Vasquez, A. (2011): "Do Realized Skewness and Kurtosis Predict the Cross-Section of Equity Returns?" CREATES Research Papers.

Anagnou-Basioudis, I., Bedendo, M., Hodges, S.D., Tompkins, R. (2005): "Forecasting Accuracy of Implied and GARCH-Based Probability Density Functions". Review of Futures Markets, 14.

Andersen, T.G., Bollerslev, T. (1998): "Answering the Skeptics: Yes, Standard Volatility Models Do Provide Accurate Forecasts". International Economic Review, 39(4), 885905.

Andersen, T.G., Bollerslev, T., Diebold, F.X., Labys, P. (2003): "Modeling and Forecasting Realized Volatility". Econometrica, 71(2), 579-625.

Ang, A., Bekaert, G., Liu, J. (2005): "Why stocks may disappoint". Journal of Financial Economics, 76(3), 471-508.

Ang, A., Hodrick, R.J., Xing, Y., Zhang, X. (2006): "The Cross-Section of Volatility and Expected Returns". The Journal of Finance, 61(1), 259-299.

Ang, A., Hodrick, R.J., Xing, Y., Zhang, X. (2009): "High idiosyncratic volatility and low returns: International and further U.S. evidence". Journal of Financial Economics, 91(1), 1-23. 
Arditti, F.D. (1967): "Risk and the Required Return on Equity". The Journal of Finance, $22(1), 19-36$.

Aït-Sahalia, Y., Brandt, M.W. (2008): "Consumption and Portfolio Choice with OptionImplied State Prices". Working Paper 13854, National Bureau of Economic Research.

Aït-Sahalia, Y., Lo, A.W. (2000): "Nonparametric risk management and implied risk aversion". Journal of Econometrics, 94(1-2), 9-51.

Avramov, D., Zhou, G. (2010): "Bayesian Portfolio Analysis". Annual Review of Financial Economics, 2(1), 25-47.

Bakshi, G., Kapadia, N. (2003): "Delta-Hedged Gains and the Negative Market Volatility Risk Premium". Review of Financial Studies, 16(2), 527-566.

Bakshi, G., Kapadia, N., Madan, D. (2003): "Stock Return Characteristics, Skew Laws, and the Differential Pricing of Individual Equity Options". The Review of Financial Studies, 16(1), 101-143.

Bakshi, G., Madan, D. (2006): "A Theory of Volatility Spreads". Management Science, 52(12), 1945-1956.

Bakshi, G., Panayotov, G., Skoulakis, G. (2011): "Improving the predictability of real economic activity and asset returns with forward variances inferred from option portfolios". Journal of Financial Economics, 100(3), 475-495.

Bali, T.G., Hovakimian, A. (2009): "Volatility Spreads and Expected Stock Returns". Management Science, 55(11), 1797-1812.

Bali, T.G., Murray, S. (2013): "Does Risk-Neutral Skewness Predict the Cross-Section of Equity Option Portfolio Returns?" Journal of Financial and Quantitative Analysis, forthcoming.

Baule, R., Korn, O., Saßning, S. (2013): "Which Beta is Best? On the Information Content of Option-Implied Betas". Working Paper Georg August Universität Göttingen.

Beckers, S. (1981): "Standard deviations implied in option prices as predictors of future stock price variability". Journal of Banking \& Finance, 5(3), 363-381.

Bekaert, G., Wu, G. (2000): "Asymmetric volatility and risk in equity markets". Review of Financial Studies, 13(1), 1-42.

Berkowitz, J. (2001): "Testing Density Forecasts, with Applications to Risk Management". Journal of Business \& Economic Statistics, 19(4), 465-474. 
Best, M.J., Grauer, R.R. (1991): "On the sensitivity of mean-variance efficient portfolios to changes in asset means: some analytical and computational results". Review of Financial Studies, 4(2), 315-342.

Binsbergen, J., Brandt, M. (2007): "Solving dynamic portfolio choice problems by recursing on optimized portfolio weights or on the value function?" Computational Economics, 29(3), 355-367.

Black, F., Scholes, M. (1973): “The Pricing of Options and Corporate Liabilities". Journal of Political Economy, 81(3), 637-654.

Blair, B.J., Poon, S.H., Taylor, S.J. (2001): "Forecasting S\&P 100 volatility: the incremental information content of implied volatilities and high-frequency index returns". Journal of Econometrics, 105(1), 5 - 26.

Bliss, R.R., Panigirtzoglou, N. (2002): "Testing the stability of implied probability density functions". Journal of Banking \& Finance, 26(2-3), 381-422.

Bliss, R.R., Panigirtzoglou, N. (2004): "Option-Implied Risk Aversion Estimates". The Journal of Finance, 59(1), 407-446.

Bollerslev, T., Gibson, M., Zhou, H. (2011): "Dynamic estimation of volatility risk premia and investor risk aversion from option-implied and realized volatilities". Journal of Econometrics, 160(1), 235-245.

Bollerslev, T., Tauchen, G., Zhou, H. (2009): "Expected Stock Returns and Variance Risk Premia". Review of Financial Studies, 22(11), 4463-4492.

Brandt, M.W. (2010): Handbook of Financial Econometrics: Chapter 5 Portfolio Choice Problems, Bd. 1 (Elsevier).

Brandt, M.W., Goyal, A., Santa-Clara, P., Stroud, J.R. (2005): "A Simulation Approach to Dynamic Portfolio Choice with an Application to Learning About Return Predictability". Review of Financial Studies, 18(3), 831-873.

Brandt, M.W., Santa-Clara, P., Valkanov, R. (2009): "Parametric Portfolio Policies: Exploiting Characteristics in the Cross-Section of Equity Returns". Review of Financial Studies, 22(9), 3411-3447.

Breeden, D.T., Litzenberger, R.H. (1978): "Prices of State-Contingent Claims Implicit in Option Prices". The Journal of Business, 51(4), 621-651.

Brennan, M.J., Schwartz, E.S., Lagnado, R. (1997): "Strategic asset allocation". Journal of Economic Dynamics and Control, 21. 
Brinkmann, F., Kempf, A., Korn, O. (2013): "Foward-Looking Measures of Higher Order Dependencies with an Application to Portfolio Selection". Working Paper Georg-AugustUniversität Göttingen und Centre for Financial Research Cologne (CFR).

Britten-Jones, M., Neuberger, A. (2000): "Option Prices, Implied Price Processes, and Stochastic Volatility". The Journal of Finance, 55(2), 839-866.

Brockett, P.L., Golden, L.L. (1987): "A Class of Utility Functions Containing All the Common Utility Functions". Management Science, 33(8), 955-964.

Busch, T., Christensen, B.J., Ørregaard Nielsen, M. (2011): "The role of implied volatility in forecasting future realized volatility and jumps in foreign exchange, stock, and bond markets". Journal of Econometrics, 160(1), 48-57.

Buss, A., Vilkov, G. (2012): "Measuring Equity Risk with Option-implied Correlations". Review of Financial Studies, 25(10), 3113-3140.

Caballé, J., Pomansky, A. (1996): "Mixed Risk Aversion". Journal of Economic Theory, $71(2), 485-513$.

Campa, J.M., Chang, P., Reider, R.L. (1998): "Implied exchange rate distributions: evidence from OTC option markets". Journal of International Money and Finance, 17(1), $117-160$.

Campbell, J.Y., Chan, Y.L., Viceira, L.M. (2003): "A multivariate model of strategic asset allocation". Journal of Financial Economics, 67(1), 41-80.

Campbell, J., Viceira, L. (2001): Strategic asset allocation: Portfolio choice for long-term investors (Oxford University Press).

Canina, L., Figlewski, S. (1993): "The informational content of implied volatility". Review of Financial Studies, 6(3), 659-681.

Carhart, M.M. (1997): "On Persistence in Mutual Fund Performance". The Journal of Finance, 52(1), 57-82.

Carr, P., Madan, D. (2001): "Optimal positioning in derivative securities". Quantitative Finance, 1(1), 19-37.

Carr, P., Wu, L. (2009): "Variance Risk Premiums". Review of Financial Studies, 22(3), $1311-1341$.

Chabi-Yo, F. (2012): "Pricing Kernels with Stochastic Skewness and Volatility Risk". Management Science, 58(3), 624-640.

Chan, L., Karceski, J., Lakonishok, J. (1999): "On portfolio optimization: forecasting covariances and choosing the risk model". Review of Financial Studies, 12(5), 937-974. 
Chang, B.Y., Christoffersen, P., Jacobs, K. (2013): "Market skewness risk and the cross section of stock returns". Journal of Financial Economics, 107(1), 46-68.

Chang, B.Y., Christoffersen, P., Jacobs, K., Vainberg, G. (2011a): "Option-Implied Measures of Equity Risk". Review of Finance.

Chang, B., Christoffersen, P., Jacobs, K. (2011b): "Forecasting with Option Implied Information". SSRN Paper, 1969863.

Chopra, V.K., Ziemba, W.T. (1993): "The effect of errors in means, variances and covariances on optimal portfolio choice". Journal of Portfolio Management, 19, 6-11.

Clarke, R., De Silva, H., Thorley, S. (2010): "Minimum Variance Portfolio Composition". SSRN Paper, 1549949.

Cochrane, J.H. (2005): Asset Pricing (Revised Edition) (Princeton University Press).

Conrad, J., Dittmar, R.F., Ghysel, E. (2013): "Ex Ante Skewness and Expected Stock Returns". The Journal of Finance, 68(1), 85-124.

Constantinides, G.M. (1982): "Intertemporal Asset Pricing with Heterogeneous Consumers and Without Demand Aggregation". The Journal of Business, 55(2), 253-267.

Cremers, M., Weinbaum, D. (2010): "Deviations from Put-Call Parity and Stock Return Predictability". Journal of Financial and Quantitative Analysis, 45(02), 335-367.

Cvitanic, J., Polimenis, V., Zapatero, F. (2008): "Optimal portfolio allocation with higher moments". Annals of Finance, 4(1), 1-28.

Daskalaki, C., Skiadopoulos, G. (2011): "Should investors include commodities in their portfolios after all? New evidence". Journal of Banking $\&$ Finance.

Day, T.E., Lewis, C.M. (1992): "Stock market volatility and the information content of stock index options". Journal of Econometrics, 52, 267-287.

DeJong, F., Schotman, P., Werker, B. (2008): "Strategic asset allocation". Netspar Panel Paper, (8).

DeMiguel, V., Garlappi, L., Nogales, F.J., Uppal, R. (2009a): “A Generalized Approach to Portfolio Optimization: Improving Performance by Constraining Portfolio Norms". Management Science, 55(5), 798-812.

DeMiguel, V., Garlappi, L., Uppal, R. (2009b): "Optimal Versus Naive Diversification: How Inefficient is the 1/N Portfolio Strategy?" Review of Financial Studies, 22(5), 1915-1953. 
DeMiguel, V., Plyakha, Y., Uppal, R., Vilkov, G. (2012): "Improving Portfolio Selection Using Option-Implied Volatility and Skewness". Journal of Financial and Quantitative Analysis, forthcoming.

Diavatopoulos, D., Doran, J.S., Fodor, A., Peterson, D.R. (2012): “The information content of implied skewness and kurtosis changes prior to earnings announcements for stock and option returns". Journal of Banking \& Finance, 36(3), 786-802.

Diris, B., Palm, F., Schotman, P. (2009): "Long-term strategic asset allocation: an out-ofsample evaluation". Working Paper.

Dittmar, R.F. (2002): "Nonlinear Pricing Kernels, Kurtosis Preference, and Evidence from the Cross Section of Equity Returns". The Journal of Finance, 57(1), 369-403.

Doron, Avramov (2002): "Stock return predictability and model uncertainty". Journal of Financial Economics, 64(3), 423-458.

Driessen, J., Maenhout, P., Vilkov, G. (2012): "Option-implied correlations and the price of correlation risk". Advanced Risk \& Portfolio Management Paper.

Driessen, L., Maenhout, P.J., Vilkov, G. (2009): "The Price of Correlation Risk: Evidence from Equity Options". The Journal of Finance, 64(3), 1377-1406.

Duan, J., Zhang, W. (2010): "Forward-looking market risk premium". SSRN Paper, 1584480 .

Ederington, L., Guan, W. (2002): "Is Implied Volatility an Informationally Efficient and Effective Predictor of Future Volatility?" Journal of Risk, Seiten 29-49.

Elton, E.J., Gruber, M.J. (1973): "Estimating the Dependence Structure of Share PricesImplications for Portfolio Selection". The Journal of Finance, 28(5), 1203-1232.

Eraker, B. (2008): "Affine General Equilibrium Models". Management Science, 54(12), 2068-2080.

Fama, E.F., French, K.R. (1993): "Common risk factors in the returns on stocks and bonds". Journal of Financial Economics, 33(1), 3-56.

Fama, E.F., MacBeth, J.D. (1973): "Risk, return, and equilibrium: Empirical tests". The Journal of Political Economy, Seiten 607-636.

Fleming, J. (1998): "The quality of market volatility forecasts implied by S\&P 100 index option prices". Journal of Empirical Finance, 5(4), 317-345.

Frost, P.A., Savarino, J.E. (1988): "For better performance: Constrain portfolio weights". The Journal of Portfolio Management, 15(1), 29-34. 
Fugazza, C., Guidolin, M., Nicodano, G. (2010): "1/N and long run optimal portfolios: results for mixed asset menus". SSRN Paper, 1533537.

Garlappi, L., Skoulakis, G. (2011): "Taylor series approximations to expected utility and optimal portfolio choice". Mathematics and Financial Economics, Seiten 1-36.

Glosten, L.R., Jagannathen, R., Runkle, D.E. (1993): "On the Relation between the Expected Value and the Volatility of the Nominal Excess Return on Stocks". The Journal of Finance, 48(5), 1779-1801.

Goetzmann, W.N., Kumar, A. (2008): "Equity Portfolio Diversification". Review of Finance, 12(3), 433-463.

Gorton, G., Rouwenhorst, K.G. (2006): "Facts and Fantasies about Commodity Futures". Financial Analysts Journal, 62(2), 47-68.

Goyal, A., Welch, I. (2008): "A Comprehensive Look at The Empirical Performance of Equity Premium Prediction". Review of Financial Studies, 21(4), 1455-1508.

Guidolin, M., Timmermann, A. (2008): "International asset allocation under regime switching, skew, and kurtosis preferences". Review of Financial Studies, 21(2), 889-935.

Harvey, C., Liechty, J., Liechty, M., Muller, P. (2010): "Portfolio selection with higher moments". Quantitative Finance, 10(5), 469-485.

Harvey, C.R., Siddique, A. (2000): "Conditional Skewness in Asset Pricing Tests". The Journal of Finance, 55(3), 1263-1295.

Hoevenaars, R., Molenaar, R., Schotman, P., Steenkamp, T. (2007): "Strategic asset allocation for long-term investors: Parameter uncertainty and prior information". LIFE working paper, Maastricht University.

Hong, Y., Tu, J., Zhou, G. (2007): "Asymmetries in Stock Returns: Statistical Tests and Economic Evaluation". Review of Financial Studies, 20(5), 1547-1581.

Huang, C., Litzenberger, R. (1988): Foundations for financial economics, Bd. 4 (NorthHolland Amsterdam).

Hull, J.C. (2006): Optionen, Futures und andere Derivate, Bd. 6 (Pearson Deutschland $\mathrm{GmbH})$.

Jackwerth, J. (2004): "Option-implied risk-neutral distributions and risk aversion". CFA Institute Research Foundation of AIMR Publications, Seiten 1-86.

Jackwerth, J. (2000): "Recovering risk aversion from option prices and realized returns". Review of Financial Studies, 13(2), 433-451. 
Jagannathan, R., Ma, T. (2003): "Risk Reduction in Large Portfolios: Why Imposing the Wrong Constraints Helps". The Journal of Finance, 58(4), 1651-1683.

Jensen, G.R., Johnson, R.R., Mercer, J.M. (2000): "Efficient use of commodity futures in diversified portfolios". Journal of Futures Markets, 20(5), 489-506.

Jiang, G.J., Tian, Y.S. (2005): "The Model-Free Implied Volatility and Its Information Content". The Review of Financial Studies, 18(4), 1305-1342.

Jondeau, E., Jurczenko, E., Rockinger, M. (2010): "Moment Component Analysis: An Illustration with International Stock Markets". Swiss Finance Institute Research Paper Series 10-43, Swiss Finance Institute.

Jondeau, E., Rockinger, M. (2001): "Gram Charlier Densities". Journal of Economic Dynamics and Control, 25(10), 1457-1483.

Jondeau, E., Rockinger, M. (2006): "Optimal Portfolio Allocation under Higher Moments". European Financial Management, 12(1), 29-55.

Jorion, P. (1986): "Bayes-Stein Estimation for Portfolio Analysis". Journal of Financial and Quantitative Analysis, 21(03), 279-292.

Jorion, P. (1995): "Predicting Volatility in the Foreign Exchange Market". The Journal of Finance, 50(2), 507-528.

Jurek, J.W., Viceira, L.M. (2011): "Optimal Value and Growth Tilts in Long-Horizon Portfolios". Review of Finance, 15(1), 29-74.

Kan, R., Zhou, G. (2007): "Optimal Portfolio Choice with Parameter Uncertainty". Journal of Financial and Quantitative Analysis, 42(03), 621-656.

Kandel, S., Stambaugh, R.F. (1996): "On the Predictability of Stock Returns: An AssetAllocation Perspective". The Journal of Finance, 51(2), 385 - 424.

Karoui, M. (2011): "Option-Implied Equity Premia and the Predictability of Stock Market Returns". SSRN Paper, 1933617.

Kempf, A., Korn, O., Saßning, S. (2012): "Portfolio Optimization Using Forward-Looking Information". SSRN Paper, 2012278.

Konstantinidi, E., Skiadopoulos, G., Tzagkaraki, E. (2008): "Can the evolution of implied volatility be forecasted? Evidence from European and US implied volatility indices". Journal of Banking \& Finance, 32(11), 2401-2411.

Kostakis, A., Panigirtzoglou, N., Skiadopoulos, G. (2011): "Market Timing with OptionImplied Distributions: A Forward-Looking Approach". Management Science, 57(7), 1231-1249. 
Kozhan, R., Neuberger, A., Schneider, P. (2013): "The Skew Risk Premium in the Equity Index Market". The Review of Financial Studies, forthcoming.

Kraus, A., Litzenberger, R.H. (1976): "Skewness Preference and the Valuation of Risk Assets". The Journal of Finance, 31(4), 1085-1100.

Kroll, Y., Levy, H., Markowitz, H.M. (1984): "Mean-Variance Versus Direct Utility Maximization". The Journal of Finance, 39(1), 47-61.

Lamoureux, C., Lastrapes, W. (1993): "Forecasting stock-return variance: toward an understanding of stochastic implied volatilities". Review of Financial Studies, 6(2), 293326.

Latané, H.A., Rendleman, R.J. (1976): "Standard Deviations of Stock Price Ratios Implied in Option Prices". The Journal of Finance, 31(2), 369-381.

Ledoit, O., Wolf, M. (2003): "Improved estimation of the covariance matrix of stock returns with an application to portfolio selection". Journal of Empirical Finance, 10(5), 603621.

Ledoit, O., Wolf, M. (2004): "A well-conditioned estimator for large-dimensional covariance matrices". Journal of Multivariate Analysis, 88(2), 365-411.

Lintner, J. (1965): "The Valuation of Risk Assets and the Selection of Risky Investments in Stock Portfolios and Capital Budgets". The Review of Economics and Statistics, $47(1), 13-37$.

Liu, X., Shackleton, M.B., Taylor, S.J., Xu, X. (2007): "Closed-form transformations from risk-neutral to real-world distributions". Journal of Banking \& Finance, 31(5), 15011520.

MacKinlay, C.A., Pástor, L. (2000): "Asset pricing models: implications for expected returns and portfolio selection". Review of Financial Studies, 13(4), 883-916.

Malz, A.M. (1997a): "Option-implied probability distributions and currency excess returns". Staff Reports 32, Federal Reserve Bank of New York.

Malz, A. (1997b): "Estimating the probability distribution of the future exchange rate from option prices". The Journal of Derivatives, 5(2), 18-36.

Markowitz, H. (1952): "Portfolio Selection". The Journal of Finance, 7(1), 77-91.

Martellini, L., Ziemann, V. (2010): "Improved Estimates of Higher-Order Comoments and Implications for Portfolio Selection". Review of Financial Studies, 23(4), 1467-1502.

Martens, M., Zein, J. (2004): "Predicting financial volatility: High-frequency time-series forecasts vis-à-vis implied volatility". Journal of Futures Markets, 24(11), 1005-1028. 
Memmel, C. (2004): Schätzrisiken in der Portfoliotheorie: Auswirkungen und Möglichkeiten der Reduktion, Bd. 34 (Josef Eul Verlag).

Merton, R. (1971): "Optimum consumption and portfolio rules in a continuous-time model". Journal of Economic Theory, 3(4), 373-413.

Merton, R.C. (1969): "Lifetime Portfolio Selection under Uncertainty: The ContinuousTime Case". The Review of Economics and Statistics, 51(3), 247-257.

Merton, R.C. (1973): "Theory of Rational Option Pricing". The Bell Journal of Economics and Management Science, 4(1), 141-183.

Merton, R.C. (1980): "On estimating the expected return on the market : An exploratory investigation". Journal of Financial Economics, 8(4), 323-361.

Michaud, R.O. (1989): "The Markowitz Optimization Enigma: Is 'Optimized' Optimal?" Financial Analysts Journal, 45(1), 31-42.

Mitton, T., Vorkink, K. (2007): "Equilibrium Underdiversification and the Preference for Skewness". Review of Financial Studies, 20(4), 1255-1288.

Mossin, J. (1966): "Equilibrium in a Capital Asset Market". Econometrica, 34(4), 768-783. ISSN 00129682.

Neuberger, A. (2012): "Realized Skewness". Review of Financial Studies, 25(11), 34233455.

Neumann, M., Skiadopoulos, G. (2012): "Predictable Dynamics in Higher Order RiskNeutral Moments: Evidence from the S\&P 500 Options". Journal of Financial and Quantitative Analysis, forthcoming.

Patton, A.J. (2004): "On the Out-of-Sample Importance of Skewness and Asymmetric Dependence for Asset Allocation". Journal of Financial Econometrics, 2(1), 130-168.

Plyakha, Y., Uppal, R., Vilkov, G. (2012): "Why Does an Equal-Weighted Portfolio Outperform Value-and Price-Weighted Portfolios?" SSRN Paper, 1787045.

Poon, S.H., Granger, C.W.J. (2003): "Forecasting Volatility in Financial Markets: A Review". Journal of Economic Literature, 41(2), 478-539.

Pástor, L. (2000): "Portfolio Selection and Asset Pricing Models". The Journal of Finance, $55(1), 179-223$.

Pástor, L., Stambaugh, R.F. (2000): "Comparing asset pricing models: an investment perspective". Journal of Financial Economics, 56(3), 335-381. 
Rehman, Z., Vilkov, G. (2012): "Risk-neutral skewness: Return predictability and its sources". SSRN Paper, 1301648.

Rosenberg, J.V., Engle, R.F. (2002): "Empirical pricing kernels". Journal of Financial Economics, 64(3), 341-372.

Samuelson, P.A. (1970): "The Fundamental Approximation Theorem of Portfolio Analysis in terms of Means, Variances and Higher Moments". The Review of Economic Studies, $37(4), 537-542$.

Saßning, S. (2012): Portfolio-Optimierung und Beta-Bestimmung unter Verwendung impliziter Informationen (Cuvillier).

Scott, R.C., Horvath, P.A. (1980): "On the Direction of Preference for Moments of Higher Order than the Variance". The Journal of Finance, 35(4), 915-919.

Shackleton, M.B., Taylor, S.J., Yu, P. (2010): "A multi-horizon comparison of density forecasts for the S\&P 500 using index returns and option prices". Journal of Banking \& Finance, 34(11), 2678-2693.

Sharpe, W.F. (2007): "Expected utility asset allocation". Financial Analysts Journal, 63, $18-30$.

Sharpe, W.F. (1963): "A Simplified Model for Portfolio Analysis". Management Science, 9(2), 277-293.

Sharpe, W.F. (1964): "Capitel Asset Prices: A Theory of Market Equlibrium under Conditions of Risk". The Journal of Finance, 19(3), 425-442.

Shimko, D. (1993): "Bounds of probability". Risk, 6(4), 33-37.

Siegel, A.F., Woodgate, A. (2007): "Performance of Portfolios Optimized with Estimation Error". Management Science, 53(6), 1005-1015.

Silvennoinen, A., Thorp, S. (2013): "Financialization, crisis and commodity correlation dynamics". Journal of International Financial Markets, Institutions and Money, 24(0), $42-65$.

Skintzi, V.D., Refenes, A.P.N. (2005): "Implied correlation index: A new measure of diversification". Journal of Futures Markets, 25(2), 171-197.

Smith, D.R. (2007): "Conditional coskewness and asset pricing". Journal of Empirical Finance, 14(1), 91 - 119.

Swidler, S., Wilcox, J.A. (2002): "Information about bank risk in options prices". Journal of Banking \& Finance, 26(5), 1033-1057. 
Tu, J., Zhou, G. (2011): "Markowitz meets Talmud: A combination of sophisticated and naive diversification strategies". Journal of Financial Economics, 99(1), 204-215.

Vilkov, G., Xiao, Y. (2012): "Option-Implied Information and Predictability of Extreme Returns". SSRN Paper, 2147437.

Xing, Y., Zhang, X., Zhao, R. (2010): "What Does the Individual Option Volatility Smirk Tell Us About Future Equity Returns?" Journal of Financial and Quantitative Analysis, 45(03), 641-662.

Zdorovenin, V., Pezier, J. (2011): "Does Information Content of Option Prices Add Value for Asset Allocation?" ICMA Centre Discussion Paper No. DP2011-03.

Ziegler, A. (2007): "Why Does Implied Risk Aversion Smile?" Review of Financial Studies, 20(3), 859-904. 